This item was submitted to Loughborough's Research Repository by the author.

Items in Figshare are protected by copyright, with all rights reserved, unless otherwise indicated.

\title{
Reverse micelle liquid-liquid extraction of a pharmaceutical product
}

PLEASE CITE THE PUBLISHED VERSION

PUBLISHER

(C) Siti H. Mohd-Setapar

\section{PUBLISHER STATEMENT}

This work is made available according to the conditions of the Creative Commons Attribution-NonCommercialNoDerivatives 4.0 International (CC BY-NC-ND 4.0) licence. Full details of this licence are available at: https://creativecommons.org/licenses/by-nc-nd/4.0/

\section{LICENCE}

CC BY-NC-ND 4.0

\section{REPOSITORY RECORD}

Mohd-Setapar, Siti H.. 2018. "Reverse Micelle Liquid-liquid Extraction of a Pharmaceutical Product". figshare. https://hdl.handle.net/2134/36143. 


\section{University Library}

L Loughborough

University

Author/Filing Title .....MOHD-SETAPAR S.H.

Class Mark

$T$

Please note that fines are charged on ALL overdue items.

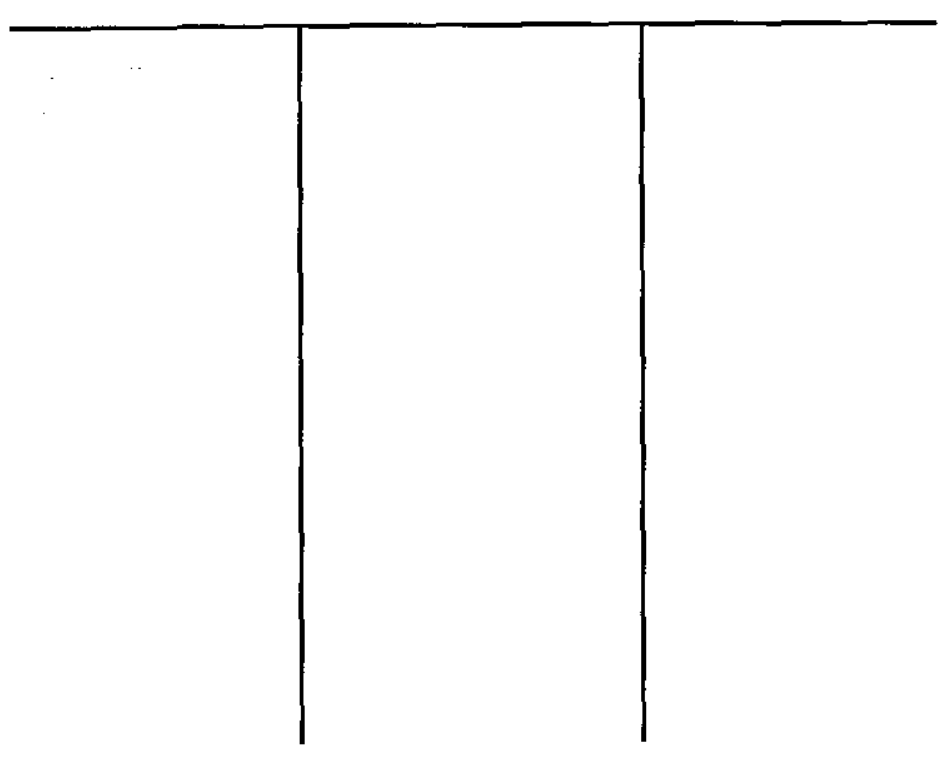





\title{
REVERSE MICELLE LIQUID-LIQUID EXTRACTION OF A PHARMACEUTICAL PRODUCT
}

\author{
Siti H. Mohd-Setapar
}

A Doctoral thesis submitted in partial fulfillment of the requirements for the award of Doctor of Philosophy of Loughborough University

\section{July 2008}

(C) Siti H. Mohd-Setapar, 2008 


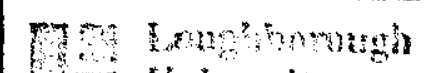

Univerngty

Pikingion bintary

Date 23/10/09

Class T

Acc 0403819105 


\section{SUMMARY}

Reverse micelle extraction has received considerable attention in recent years due to its ability to selectively solubilise solutes from an aqueous phase, and in the case of biomolecules to maintain their biological activities. This thesis reports the results from studies on the extraction of penicillin $G$ from aqueous solution (forward extraction) and from the reverse micelle to a new aqueous solution (backward extraction). The extraction is influenced by the initial penicillin $G$ concentration, the salt type and concentration in the aqueous phase, $\mathrm{pH}$, and surfactant concentration. The results show that penicillin is an interfacially active compound that interacts with AOT, with the interfacial association being dependent on both $\mathrm{pH}$ and surfactant concentration. When the penicillin to surfactant concentration ratio $[P]_{a q} /[S]$ is high precipitation of the penicillin occurs. The distribution coefficient favours transfer of the penicillin into the reverse micelle at moderate AOT concentrations. The distribution coefficient at infinite dilution, $K_{f w}^{\infty}$, is shown to be a function of both $\mathrm{pH}$ and surfactant concentration; similar trends in the value of $K_{f w}^{\infty}$ were observed at different $\mathrm{pH}$ values. $K_{f w}^{\infty}$ decreases as the surfactant concentration is increased. Despite successful results in the forward extraction, the extraction of penicillin $\mathrm{G}$ in the backward process was found to be less efficient. The overall yield, when taking the forward and backward processes together, is consequently lower than might be expected - that is, the penicillin recovered from the backward process is less than the initial mass of penicillin $G$ present in the aqueous phase before forward extraction. 
For Munif 


\section{ACKNOWLEDGMENTS}

This work could not have been completed without the assistance of several people whom I would hereby like to acknowledge.

First and foremost I would like to thank my supervisors, Professor R.J. Wakeman and Dr. E.S. Tarleton, for their support throughout this project. I am grateful for the opportunity to have work with them, and for the many useful discussions and advice that were invaluable to the accomplishment of this thesis.

I would like to thank the following people for their important contribution: Dave Smith and Shawn Creedon from the Department laboratory for their help with the construction of the experimental equipment. I am also grateful to the many individuals who contributed in so many different ways to the completion of his project. Special mentions are merited to Jolius Gimbun, Rushdi Abu Bakar, Azizul Buang and Heema Unakdat for their help and support, and Kankanit Khwanpruk for her sincere friendship throughout this challenging journey.

I also express my sincere gratitude to my employer and financial sponsor, Universiti Teknologi Malaysia that helped underwrite the whole cost of my study.

I am highly grateful and express my profound appreciation to my parents Mohd Setapar Zali and Siti Hawa Ibrahim, my sisters and brother; Azmah, Afiah, Shuhadah, Rafidah and Izzat, for their continued love, support, prayers, encouragements and inspiration all throughout my life.

Special thank to my husband Sarajul Fikri Mohamed for being so loving, patient and supportive at all times. Also appreciation to our lovely son Munif Afifi (his welcome arrival is during the research period) for his patient and understanding. 


\section{TABLE OF CONTENTS}

SUMMARY i

DEDICATION ii

ACKNOWLEDGMENT

TABLE OF CONTENTS _ iv

LIST OF FIGURES viii

LIST OF TABLES Xii

LIST OF SYMBOLS Xiii

LIST OF ACRONYMS xvii

\section{CHAPTER ONE: INTRODUCTION}

1.1 Introduction 1

1.2 Background 1

1.3 Objectives 3

1.4 Scope of Work 4

1.5 Thesis Outline 5

\section{CHAPTER TWO: LITERATURE REVIEW AND CONCEPTS}

$\begin{array}{lll}2.1 & \text { Introduction } & 7\end{array}$

2.2 Separation Methods in the Pharmaceutical Industry 7

2.2.1 Conventional Solvent Extraction 10

2.2.2 Liquid Membrane Extraction 12

2.2.3 Aqueous Two-Phase System 14

$\begin{array}{lll}2.2 .4 & \text { Chromatography } & 15\end{array}$ 
2.3 Fundamentals of Reverse Micelle 15

$2.4 \quad$ Formation of Reverse Micelle $\quad 19$

$\begin{array}{lll}2.5 \text { Surfactants } & 21\end{array}$

2.5.1 Surfactant Structure 22

2.5.2 Categories of Surfactant 22

2.5.3 Critical Micelle Concentration (CMC) 24

2.6 Solubilisation in Reverse Micelle 26

2.7 Biomolecule Partitioning in Reverse Micelle 29

2.7.1 Aqueous Phase $\mathrm{pH} \quad 30$

2.7.2 Salt Concentration and Type $\quad 32$

2.7.3 Surfactant Concentration 34

2.7.4 Biomolecule Size and Concentration 35

2.7.5 Solvent Type 36

2.7.6 Other Factors that Influence Partitioning 38

2.7.7 Backward Extraction Process 38

2.7.8 Thermodynamic Framework of Partitioning 40

$\begin{array}{lll}2.8 & \text { Conclusions } & 43\end{array}$

CHAPTER THREE: EXPERIMENTAL MATERIALS AND METHODS

3.1 Introduction 44

3.2 Materials 44

3.2.1 Penicillin G 45

3.3 Feed Solution Characteristics 46

3.3.1 Mass and Concentration of Penicillin G 46

3.3.2 Surface Tension 48

3.3.3 Water Content 50

$3.3 .4 \mathrm{pH} \quad 52$

3.4 Forward Extraction Experimental Procedure 52

3.5 Backward Extraction Experimental Procedure 54 
3.6 Penicillin G Content Determination 56

3.6.1 Calibration Curve 56

3.6.2 Calculating Penicillin G Content 57

$\begin{array}{lll}3.7 & \text { Conclusions } & 59\end{array}$

\section{CHAPTER FOUR: RESULTS AND DISCUSSION - FORWARD EXTRACTION}

4.1 Introduction 61

4.2 Sample Characterisation - Critical Micelle Concentration 61

4.3 Interfacial Association and Phase Volume Changes 63

4.4 Partitioning of Penicillin G between Phases 68

4.5 Other Effects on the Solubilisation of Penicllin G 74

$\begin{array}{lll}4.5 .1 & \text { Salt Concentration } & 74\end{array}$

$\begin{array}{lll}\text { 4.5.2 Salt Type } & 76\end{array}$

4.6 Reverse Micelle Formation and Water Content 77

4.7 Thermodynamic Framework for Solubilisation 81

4.7.1 Distribution Coefficient at Infinite Dilution 82

4.7.1.1 Data Fitted with the Langmuir Model 83

4.7.1.2 Data Fitted with the Brandani Model 86

$\begin{array}{lll}4.8 \text { Conclusions } & 90\end{array}$

CHAPTER FIVE: RESULTS AND DISCUSSION - BACKWARD EXTRACTION

$\begin{array}{lll}5.1 & \text { Introduction } & 92\end{array}$

5.2 Factors Affecting Backward Extraction 93

5.2.1 Additional Volume of the Backward Aqueous Phase 95

5.2.2 Effect of Salt Concentration 97

$\begin{array}{lll}5.2 .3 & \text { Effect of } \mathrm{pH} & 100\end{array}$ 
5.2.4 Effect of Initial Mass of Penicillin G and Surfactant Concentration

5.3 Thermodynamic Framework for Solubilisation

5.3.1 Distribution Coefficient at Infinite Dilution $\left(K_{b w}^{\infty}\right) \quad 107$

5.3.1.1 Data Fitted with the Langmuir Model $\quad 107$

5.3.1.2 Data Fitted with the Brandani Model $\quad 110$

5.4 Suggestions for Improving Backward Extraction 114

$\begin{array}{lll}5.5 \text { Conclusions } & 116\end{array}$

\section{CHAPTER SIX: CONCLUSIONS AND RECOMMENDATIONS}

$\begin{array}{lll}6.1 & \text { Introduction } & 118\end{array}$

$\begin{array}{lll}6.2 & \text { Overall Conclusions } & 118\end{array}$

$\begin{array}{ll}6.3 \text { Constraints of the Study } & 120\end{array}$

$\begin{array}{lll}6.4 & \text { Recommendations for Future Work } & 121\end{array}$

$\begin{array}{lr}\text { REFERENCES } & 123\end{array}$

$\begin{array}{ll}\text { APPENDICES } & 139\end{array}$

Appendix 1 Sample Calculation of the Final Concentration/Mass of

Penicillin $\mathrm{G}$ in the Aqueous Phase, $[P]_{a q . f . f w}$ and the Organic phase, $[P]_{r m+o f f w} \quad 140$

Appendix 2 Experimental Results for Forward Extraction 143

Appendix 3 Experimental Results for Forward Extraction 165

Appendix 4 Thermodynamic Framework for Forward Extraction 179

Appendix 5 Thermodynamic Framework for Backward Extraction 186

Appendix 6 List of Publications Arising from the Research 193 


\section{LIST OF FIGURES}

Figure 2.1 Schematic of the solvent extraction method used in industry.

Figure 2.2 Liquid membranes: (a) emulsion liquid membrane,

(b) supported liquid membrane.

$\begin{array}{lll}\text { Figure 2.3 Schematic representation of a reverse micelle. } & 17\end{array}$

$\begin{array}{lll}\text { Figure 2.4 Structure of a surfactant. } & 17\end{array}$

Figure 2.5 Aggregation of surfactants; (a) micelles, and (b) reverse micelle. 20

Figure 2.6 Effect of surfactant concentration on several solution properties, and the consequences of micelle formation on these properties. 25

Figure 2.7 Representation of few surfactant structures that are thought to form at concentrations greater than the CMC 26

Figure 3.1 Structure of sodium di-2-ethylhexylsulphosuccinate (AOT). 45

Figure 3.2 Structure of Penicillin G. 45

$\begin{array}{lll}\text { Figure } 3.3 \quad \text { Nitrogen analyzer. . } & 47\end{array}$

Figure 3.4 Schematic plot of surface tension vs. concentration of surfactant. 49

$\begin{array}{lll}\text { Figure } 3.5 & \text { Surface tension unit. } & 50\end{array}$

Figure 3.6 Karl Fischer titration unit. $\quad 51$

Figure $3.7 \quad$ Forward extraction by reverse micelle. 53

Figure 3.8 Backward extraction from reverse micelle. 54

Figure 3.9 Flow chart of the experimental procedures for forward and backward extraction. $\quad 55$

Figure 3.10 Calibration curve of penicillin G. 57

Figure 4.1 Effect of AOT concentration on the surface tension of isooctane with and without penicillin G. $\quad 62$

Figure 4.2 Schematic diagram of the reverse micelle phase. 64

Figure 4.3 Phase sequence after 1 week of the extraction process. 64 
Figure 4.4 Additional volume of the organic phase at different AOT concentrations.

Figure 4.5 Final penicillin $\mathrm{G}$ concentration in the aqueous phase plotted against the initial penicillin $\mathrm{G}$ concentration before extraction at $23^{\circ} \mathrm{C}$ and [KCl] $10 \mathrm{~g} / 1$.

Figure 4.6 Fractional transfer of penicillin $G$ plotted against the fractional water transfer to the reverse micelle organic phase at different $\mathrm{pH}$ values and AOT concentrations.

Figure 4.7 Concentration of penicillin $\mathrm{G}$ in the organic phase at different AOT concentrations.

Figure 4.8 Distribution of penicillin $\mathrm{G}$ between the reverse micelle in the organic phase, $[P]_{m+o . f . f w}$ and the aqueous phase, $[P]_{\text {aq.f.fw }}$.

Figure 4.9 Effects of $\mathrm{pH}$ and AOT concentration on the mass of penicillin $\mathrm{G}$ extracted into the reverse micelle phase.

Figure 4.10 Effects of salt concentration on the concentration of penicillin $\mathrm{G}$ extracted into the reverse micelle phase.

Figure 4.11 Effects of salt type on the concentration of penicillin $\mathrm{G}$ extracted into the reverse micelle phase.

Figure 4.12 Effect of AOT concentration on water uptake into the reverse micelle phase presented as (a) water content, and (b) molar ratio of water to AOT, $W_{o}$.

Figure 4.13 Variation of the distribution coefficient at infinite dilution for forward extraction $\left(K_{E . f_{w}}^{\infty}\right)$ with AOT concentration and $\mathrm{pH}$.

Figure 4.14 Isotherms of penicillin $\mathrm{G}$ at $\mathrm{pH} 7.6$ and 1.65.

Figure 4.15 Langmuir model fitted to experimental data at $\mathrm{pH} 7.6$ and $[S]=88 \mathrm{~g} / 1$.

Figure 4.16 Partition coefficient at infinite dilution of Langmuir model for forward extraction, $a_{L . f w}\left(=K_{L . f w}^{\infty}\right)$ and $b_{L . f w}$ values at different $\mathrm{pH}$ and surfactant concentration.

Figure 4.17 Brandani model fitted to experimental data at $\mathrm{pH} 7.6$ and 
$[S]=88 \mathrm{~g} / \mathrm{l}$.

Figure 4.18 Partition coeffiecient at infinite dilution of Brandani model

for forward extraction, $a_{B . f w} \cdot b_{f w}[S]\left(=K_{B . f w}^{\infty}\right)$ and $b_{B . f w}$ values

at different $\mathrm{pH}$ and surfactant concentration.

Figure 5.1 The effect of backward extraction time on the mass of

penicillin $\mathrm{G}$ in the backward aqueous phase.

Figure 5.2. Effect of settling time on the mass of penicillin $\mathrm{G}$ in the aqueous phase for backward extraction process.

Figure 5.3 Additional volume of the backward aqueous phase at different surfactant concentrations.

Figure 5.4 Overall volume changing during the forward and backward extraction processes.

Figure 5.5 Effect of salt concentrations on the mass of penicillin G in the backward aqueous phase.

Figure 5.6 Effect of $\mathrm{pH}$ on the mass of penicillin $\mathrm{G}$ in the backward aqueous phase.

Figure 5.7 Effect of initial mass of penicillin $\mathrm{G}$ before forward extraction to the mass of penicillin $G$ in the backward aqueous phase.

Figure 5.8 Effect of initial mass of penicillin $G$ before backward extraction to the mass of penicillin $G$ in the backward aqueous phase.

Figure 5.9 Overall extraction of penicillin $G$.

Figure 5.10 Removal efficiency of penicillin $\mathrm{G}$ at different AOT concentrations.

Figure 5.11 Variation of the distribution coefficient at infinite dilution from experimental data for backward extraction $\left(K_{E . b w}^{\infty}\right)$ with AOT concentration and $\mathrm{pH}$.

Figure 5.12 Langmuir model fitted to experimental data at $\mathrm{pH} 7.6$ and $[S]=88 \mathrm{~g} / \mathrm{l}$ (backward extraction).

Figure 5.13 Partition coeffiecient at infinite dilution of Langmuir model for backward extraction, $a_{L . b w}\left(=K_{L . b w}^{\infty}\right)$ and $b_{L . f w}$ values at 
different $\mathrm{pH}$ and surfactant concentration.

Figure 5.14 Brandani model fitted to experimental data at $\mathrm{pH} 7.6$ and $[S]=88 \mathrm{~g} / 1$ for backward extraction.

Figure 5.15 Distribution coefficient at infinite dilution of Brandani model for backward extraction, $K_{B . b w}^{\infty}\left(=a_{B . b w} b_{B . b w}[S]\right)$ and $b_{B . b w}$ values at different $\mathrm{pH}$ and surfactant concentration, $[S]$. 


\section{LIST OF TABLES}

Table 2.1 Features of separation methods in the pharmaceutical industry. 16

$\begin{array}{lll}\text { Table 2.2 Range of solvents used. } & 37\end{array}$

Table 2.3 Extraction of SBP employing different organic solvents as feed phases.

Table 4.1 $\quad a_{L . f w}\left(=K_{L . f w}^{\infty}\right)$ and $b_{L . f w}$ values at different $\mathrm{pH}$ and surfactant concentration from Langmuir model for forward extraction. 86

Table 4.2 Comparison of distribution coefficient, $K^{\circ}$ between experimental results, Langmuir model and Brandani model.

Table 5.1 $P_{r m+o . i . b w}$ values at different parameters during forward extraction.

Table 5.2 Comparison of distribution coefficient at infinite dilution for backward extraction $\left(K_{b w}^{\infty}\right)$ between experimental results, Langmuir model and Brandani model.

Table 5.3 Efficiency of backward extraction as reported by several authors. 


\section{LIST OF SYMBOLS}

\begin{tabular}{|c|c|}
\hline$a_{\text {AOT }}$ & Surface area of the surfactant head group $\left(\mathrm{m}^{2}\right)$ \\
\hline$a_{B}$ & Adjustable parameter in Brandani equation (dimensionless) \\
\hline$a_{B . b w}$ & $\begin{array}{l}\text { Adjustable parameter in Brandani equation for backward extraction } \\
\text { (dimensionless) }\end{array}$ \\
\hline$a_{B, f w}$ & $\begin{array}{l}\text { Adjustable parameter in Brandani equation for forward extraction } \\
\text { (dimensionless) }\end{array}$ \\
\hline$a_{L}$ & Adjustable parameter in Langmuir equation (dimensionless) \\
\hline$a_{L, b w}$ & $\begin{array}{l}\text { Adjustable parameter in Langmuir equation for backward extraction } \\
\text { (dimensionless) }\end{array}$ \\
\hline$a_{L, f w}$ & $\begin{array}{l}\text { Adjustable parameter in Langmuir equation for forward extraction } \\
\text { (dimensionless) }\end{array}$ \\
\hline$b_{B}$ & Adjustable parameter in Brandani equation ( $1 / \mathrm{g})$ \\
\hline$b_{B . b w}$ & Adjustable parameter in Brandani equation for backward extraction $(1 / \mathrm{g})$ \\
\hline$b_{B, f w}$ & Adjustable parameter in Brandani equation for forward extraction $(1 / g)$ \\
\hline$b_{L}$ & Adjustable parameter in Langmuir equation $(1 / \mathrm{g})$ \\
\hline$b_{L . b w}$ & Adjustable parameter in Langmuir equation for backward extraction $(1 / \mathrm{g})$ \\
\hline$b_{L f f w}$ & Adjustable parameter in Langmuir equation for forward extraction $(1 / \mathrm{g})$ \\
\hline$\left[\mathrm{H}_{2} \mathrm{O}\right]$ & Concentration of water $(\mathrm{g} / \mathrm{l})$ \\
\hline$K_{b w}$ & $\begin{array}{l}\text { Distribution coefficient of backward extraction (dimensionless) } \\
=[P]_{a q . f . b w} /[P]_{m+o . f . b w}\end{array}$ \\
\hline
\end{tabular}

$K_{B . b w} \quad$ Distribution coefficient of Brandani equation for backward extraction (dimensionless)

$=[P]_{\text {aq.f.bw }} /[P]_{r m+o . f . b w}=\left(a_{B . b w} b_{B . b w}[S]\right) /\left(1+b_{B . b w}[P]_{m+o . f . b w}\right)$

$K_{B . b w}^{\infty} \quad$ Distribution coefficient at infinite dilution of Brandani equation for backward extraction (dimensionless), $=a_{B, b w} b_{B . b w}[S]$ 
$K_{B . f w} \quad$ Distribution coefficient of Brandani equation for forward extraction (dimensionless),

$$
=[P]_{m+o f f . f_{w}} /[P]_{a g . f . f_{w}}=\left(a_{B . f w} b_{B . f w}[S]\right) /\left(1+b_{B . f w}[P]_{a q . f . f w}\right)
$$

$K_{B . f w}^{\infty} \quad$ Distribution coefficient at infinite dilution of Brandani equation for forward extraction (dimensionless), $=a_{B . f w} b_{B . f w}[S]$

$K_{E . b w} \quad$ Distribution coefficient of backward extraction for experimental data (dimensionless), $=[P]_{a q . f . b w} /[P]_{m+o . f . b w}$

$K_{E . b w}^{\infty} \quad$ Distribution coefficient at infinite dilution of backward extraction from experimental data (dimensionless),

$=[P]_{a q . f . b w} /[P]_{m+o . f . b w},[P]_{m+o . f . b w} \rightarrow 0$

$K_{E . f w} \quad$ Distribution coefficient of forward extraction foe experimental data (dimensionless), $=[P]_{m+o . f . f w} /[P]_{\text {aq.f.fw }}$

$K_{E . f w}^{\infty} \quad$ Distribution coefficient at infinite dilution of forward extraction from experimental data (dimensionless), $=[P]_{r m+o . f . f w} /[P]_{a q . f . f w},[P]_{a q . f . f w} \rightarrow 0$

$K_{f w} \quad$ Distribution coefficient of forward extraction (dimensionless) $=[P]_{r m+p . f . f w} /[P]_{a q . f . f w}$

$K_{L, b w} \quad$ Distribution coefficient of Langmuir equation for backward extraction (dimensionless), $=[P]_{a q . f . b w} /[P]_{r m+o . f . b w}=\left(a_{L . b w}\right) /\left(1+b_{L . b w}[P]_{m+o . f . b w}\right)$

$K_{L . b w}^{\infty} \quad$ Distribution coefficient at infinite dilution of Langmuir equation for backward extraction (dimensionless), $=a_{L . b w}$

$K_{L . f w} \quad$ Distribution coefficient of Langmuir equation for forward extraction (dimensionless), $=[P]_{r m+o . f . f w} /[P]_{a q . f . f w}=\left(a_{L . f w}\right) /\left(1+b_{L . f w}[P]_{a q . f . f w}\right)$

$K_{L . f w}^{\infty} \quad$ Distribution coefficient at infinite dilution of Langmuir equation for forward extraction (dimensionless)

$=[P]_{a q . f . b w} /[P]_{m+o . f . b w}=\left(a_{L . b w}\right) /\left(1+b_{L . b w}[P]_{m+o . f . b w}\right)$

$M_{\text {acid }} \quad$ Molarity of acid (mole/l) 


\begin{tabular}{|c|c|}
\hline$\% N$ & Nitrogen percentage \\
\hline$P$ & Mass of penicillin $\mathrm{G}(\mathrm{mg})$ \\
\hline$P_{a q f f}$ & Final mass of penicillin $\mathrm{G}$ in the aqueous phase $(\mathrm{mg})$ \\
\hline$P_{a q f . b w}$ & Final mass of penicillin $\mathrm{G}$ in the backward extraction aqueous phase (mg) \\
\hline$P_{a q f f w}$ & Final mass of penicillin $\mathrm{G}$ in the forward extraction aqueous phase (mg) \\
\hline$P_{a q . i . b w}$ & Initial mass of penicillin $\mathrm{G}$ in the backward extraction aqueous phase $(\mathrm{mg})$ \\
\hline$P_{a q . i . f w}$ & Initial mass of penicillin $\mathrm{G}$ in the forward extraction aqueous phase $(\mathrm{mg})$ \\
\hline pI & Isoelectric point \\
\hline$P_{r m+o . i b w}$ & $\begin{array}{l}\text { Initial mass of penicillin } \mathrm{G} \text { in the reverse micellar phase before backward } \\
\text { extraction }(\mathrm{mg})\end{array}$ \\
\hline$P_{m+o . f}$ & Final mass of penicillin $\mathrm{G}$ in reverse micelle $(\mathrm{mg})$ \\
\hline$P_{r m+o f f b w}$ & $\begin{array}{l}\text { Final mass of penicillin } \mathrm{G} \text { in the reverse micellar phase after backward } \\
\text { extraction }(\mathrm{mg})\end{array}$ \\
\hline$P_{s}$ & Mass of penicillin $\mathrm{G}$ attached to surfactant (mg) \\
\hline$P_{s . f}$ & Final mass of penicillin $\mathrm{G}$ attached to surfactant $(\mathrm{mg})$ \\
\hline$P_{T}$ & Total mass of penicillin $\mathrm{G}$ in whole solution $(\mathrm{mg})$ \\
\hline$P_{w p}$ & Mass of penicillin $\mathrm{G}$ in water pool (mg) \\
\hline$[P]_{a q . i}$ & Initial concentration of penicillin $\mathrm{G}$ in aqueous phase $(\mathrm{g} / \mathrm{l})$ \\
\hline$[P]_{a q f f}$ & Final concentration of penicillin $\mathrm{G}$ in aqueous phase $(\mathrm{g} / \mathrm{l})$ \\
\hline$[P]_{\text {aq.i.bw }}$ & $\begin{array}{l}\text { Initial concentration of penicillin } \mathrm{G} \text { in backward extraction aqueous phase } \\
(\mathrm{g} / \mathrm{l})\end{array}$ \\
\hline$[P]_{\text {aq.i.fw }}$ & $\begin{array}{l}\text { Initial concentration of penicillin } G \text { in forward extraction aqueous phase } \\
(\mathrm{g} / \mathrm{l})\end{array}$ \\
\hline$[P]_{a q . f . b w}$ & $\begin{array}{l}\text { Final concentration of penicillin } \mathrm{G} \text { in backward extraction aqueous phase } \\
(\mathrm{g} / \mathrm{l})\end{array}$ \\
\hline$[P]_{a q . f f w}$ & $\begin{array}{l}\text { Final concentration of penicillin } \mathrm{G} \text { in forward extraction aqueous phase } \\
(\mathrm{g} / \mathrm{l})\end{array}$ \\
\hline$[P]_{m+o f . b w}$ & $\begin{array}{l}\text { Final concentration of penicillin } \mathrm{G} \text { in reverse micelle during backward } \\
\text { extraction }(\mathrm{g} / \mathrm{l})\end{array}$ \\
\hline$[P]_{m+o f f f w}$ & $\begin{array}{l}\text { Final concentration of penicillin } \mathrm{G} \text { in reverse micelle during forward } \\
\text { extraction }(\mathrm{g} / \mathrm{l})\end{array}$ \\
\hline
\end{tabular}


$[P]_{s . f} \quad$ Final concentration of penicillin $\mathrm{G}$ attached to surfactant $(\mathrm{mg} / \mathrm{ml})$

$\left[P_{r}\right]_{a q f}$ Final concentration of protein in aqueous phase $(\mathrm{g} / \mathrm{l})$

$r_{m} \quad$ Reverse micelle radius (nm)

$S \quad$ Volume of surfactant (ml)

[S] Concentration of surfactant $(\mathrm{g} / \mathrm{l})$

$t_{a} \quad$ Fractional biomolecule transfer (dimensionless)

$t_{w} \quad$ Fractional water transfer (dimensionless)

$V_{a q} \quad$ Volume of aqueous phase (ml)

$V_{a q . i} \quad$ Initial volume of aqueous phase $(\mathrm{ml})$

$V_{a q . f} \quad$ Final volume of aqueous phase (ml)

$V_{a q . f b w} \quad$ Final volume of the backward aqueous phase $(\mathrm{ml})$

$V_{\text {aq.ffi }} \quad$ Final volume of the forward aqueous phase (ml)

$V_{H 2 O} \quad$ Volume of a water molecule $\left(\mathrm{nm}^{3}\right)$

$V_{r} \quad$ Volume ratio (dimensionless)

$V_{r m+o} \quad$ Volume of reverse micelle $(\mathrm{ml})$

$V_{r m+o . f} \quad$ Volume of reverse micelle organic phase (ml)

$V_{r m+o . f . b w} \quad$ Final volume of reverse micelle in backward extraction (ml)

$V_{r m+o f f w} \quad$ Final volume of reverse micelle in forward extraction (ml)

$V_{r m+o . i} \quad$ Initial volume of reverse micelle $(\mathrm{ml})$

$V_{s . i} \quad$ Initial volume of surfactant in the reverse micelle (ml)

$V_{\text {titrant }} \quad$ Volume of titrant solution added into sample (ml)

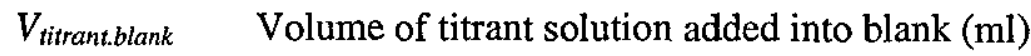

$V_{w t} \quad$ Volume of water transfer (ml)

$W_{o} \quad$ Molar ratio of water to surfactant (dimensionless)

$[W]_{a q f} \quad$ Final concentration of water in aqueous phase $(\mathrm{mg} / \mathrm{ml})$

$\Lambda_{12} \quad$ Wilson parameter (dimensionless)

$\Lambda_{21} \quad$ Wilson parameter (dimensionless)

$\theta_{1} \quad$ Fractional coverage of the protein molecules

$\ell_{s} \quad$ Density of surfactant $(\mathrm{g} / \mathrm{ml})$ 


\section{LIST OF ACRONYMS}

AOT Sodium di-2-ethylhexyl sulfosuccinate

ATPS Aqueous two-phase system

BP Boiling point

BSA Bovine serum albumin

CAC Critical alcohol concentration

CMC Critical micelle concentration

DLIPA Dilinoleyl phosphoric acid

DNA Deoxyribonucleic acid

DOLPA Dioleylphosphoric acid

CTAB Cetyl trimethyl ammonium bromide

ELM Emulsion liquid membrane

HPLC High performance liquid chromatography

PC Paper chromatography

TBP Tributyl phosphate

TLC Thin layer chromatography

TRPO Trialkyl phosphine oxide

SBP Soybean peroxidase

SLM Supported liquid membrane

6-APA 6-aminopenicillanic acid 


\section{CHAPTER ONE}

\section{Introduction}

\subsection{Introduction}

This chapter introduces the context for the research. A brief overview of the background to the research is followed by the objectives and scope of the research, along with a description of the thesis structure.

\subsection{Background}

In recent years there has been concern about efficient methods that can continuously separate pharmaceutical products such as antibiotics and that can be scaled-up easily. The reason for this is due to the fact that the separation of the product from a fermentation broth is troublesome because of the more dilute concentrations compared with many other chemical processes (Soto et al., 2005). The extraction is a challenging task since the broth contains a number of impurities and other substances along with the desired product. The production of antibiotics is one of the most important technologies in today's pharmaceutical industry and penicillin G, also known as benzyl penicillin, is a widely used antibiotic that has also been used as a raw material for semisynthetic penicillin (Yang and Cussler, 2000; Elander, 2003).

Solvent extraction (liquid-liquid extraction) is the most common method used in downstream processing of antibiotics due to its effectiveness and favourable economics (Soto et al., 2005). The process is a well known technique which is readily scaleable and can be operated on a continuous basis (Regalado et al., 1994; Hasmann et al., 2007). Despite the efficiency, solvent extraction has several difficulties which can cause problems such as final product contamination, low 
extraction yield, clogging of equipment, and high solvent losses (Wang et al., 1995; Nabais and Cardoso, 1999; Yang and Cussler, 2000). Alternative separation techniques have been studied to overcome the difficulties experienced with conventional solvent extraction for penicillin $\mathrm{G}$ which include ultrafiltration and nanofiltration (Nabais and Cardoso, 1999; Tessier et al., 2005), liquid surfactant membranes (Hano et al., 1994), and reactive extraction (Yang and Cussler, 2000). The yield obtained from these methods still comparatively lower than the conventional solvent extraction method.

This thesis is concerned with a novel method for antibiotic separation called reverse micelle extraction. Separation processes using reverse micelle are based on conventional liquid-liquid extraction techniques. They are easy to scale up, have a high efficiency, can be made selective, have a low energy demand, and can be operated continuously (Carlson and Nagarajan, 1992; Hu and Gulari, 1996; Ono et al., 1996; Chang et al., 1997; Kinugasa et al., 2003; Naoe et al., 2004; Kyung et al., 2005; Mathew and Juang, 2007). Reverse micelle techniques can also offer moderate thermal conditions for processing of biomaterials (Ichikawa et al., 1992; Tessier et al., 2005). The method is effectively a solvent extraction using reverse micelle as the extractant (Kinugasa et al., 2003).

Reverse micelle, also known as water-in-oil microemulsion, consist of three components: amphiphilic surfactant molecules, an aqueous phase, and a non-polar organic solvent. The polar heads of the surfactant molecules are directed towards the interior of a water-containing sphere, whereas the aliphatic tails are oriented toward the non-polar organic phase. Reverse micelle have the ability to extract a variety of biomolecules such as proteins (Brandani et al., 1996; Goto et al., 1998; Kinugasa et al., 2003; Bong et al., 2004; Jun et al., 2004; Kyung and Jee, 2005; Pileni, 2006) and enzymes (Bong et al., 2004; Jun et al., 2004) into nanometre-size water pools surrounded by a monolayer of surfactant.

To date most research on the purification of antibiotic molecules has focussed on those dissolved in an aqueous solvent. For example, Nabais and Cardoso (1999) studied the purification of penicillin $\mathrm{G}$ from fermentation broth by ultrafiltration, and Hu and Gulari (1996) studied the extraction of aminoglycoside antibiotics such as 
neomycin and gentamycin using reverse micelle. These authors did not give consideration to modelling of antibiotic partitioning between the phases, although thermodynamic studies are important for designing downstream processing (Haghtalab and Osfouri, 2003). Whilst thermodynamic models have been applied to protein solubilisation into reverse micelle (Woll and Hatton, 1989; Bruno et al., 1990; Leodidis and Hatton, 1990; Brandani et al., 1994; Rabie and Vera, 1998; Ashrafizadeh and Khoshkbarchi, 1998; Haghtalab and Osfouri, 2003), there does not seem to have been any thermodynamic studies of penicillin $\mathrm{G}$ solubilisation.

The apparent success of research on protein extraction from the aqueous phase using reverse micelle provides motivation to study the solubilisation of penicillin $G$, and in this work the parameters that affect solubilisation of penicillin $G$ molecules into reverse micelle that lie in the organic phase (forward extraction) and the release of penicillin $G$ from the reverse micelle into a fresh aqueous phase (backward extraction) are investigated. Sodium di(2-ethylhexyl)sulfosuccinate (AOT) was chosen as the surfactant and isooctane as the organic solvent. This system has well known advantages especially in relation to the ease of reverse micelle formation and its stability in comparison to other systems (Krieger et al., 1997). The Kjeldahl method was found to be the most convenient method to determine the mass of penicillin $G$ in solution after the extraction process since penicillin was the only nitrogen containing component in the experiments. Common analytical methods such as UV-Vis spectrophotometry cannot be used as the penicillin $\mathrm{G}$ molecule has many $\mathrm{C}=\mathrm{O}$ bonds which are sufficiently weak to make it difficult to absorb at any particular wavelength. Using the Kjeldahl method, the nitrogen content is proportional to the mass of penicillin $\mathrm{G}$.

\subsection{Objectives}

The principal objective of this study is to investigate the potential of using reverse micellar extraction for the separation of penicillin $\mathrm{G}$ from an aqueous phase into an organic phase (the forward extraction) and the subsequent transfer into a new aqueous phase (the backward extraction). 
The specific research objectives are:

1) To review the advantages of reverse micelles and to compare the system with other methods.

2) To investigate the parameters and the optimum conditions that influence penicillin $\mathrm{G}$ extraction for both forward and backward process.

3) To interpret the experimental data from both forward and backward extraction with thermodynamic models.

\subsection{Scope of Work}

The reverse micelle organic solutions were characterised using surface tension and water content measurements. The surface tension measurements were carried out to determine the critical micelle concentration (CMC) of reverse micelle with and without penicillin G. The water content of reverse micelle was measured to determine the amount of water that lies in the reverse micelle of the organic phase before and/or after the extraction process using a Karl Fisher titration method. The aqueous solutions were characterised using the Kjeldahl method in order to determine the nitrogen content corresponding to the penicillin $G$ content in the aqueous solutions after extraction. The penicillin $\mathrm{G}$ content in the reverse micelle organic phase was calculated from a mass balance over the experiment.

The effects of surfactant concentration, initial penicillin $\mathrm{G}$ concentration, $\mathrm{pH}$, salt concentration and type, water content of the reverse micelle organic phase for forward and backward extraction of penicillin $\mathrm{G}$ were investigated. Thermodynamic models developed from the Langmuir model were proposed by Brandani et al. (1994) which describe the adsorption of biomolecules by active sites of reverse micelle. The experimental data have been interpreted using the Langmuir and Brandani models. 


\subsection{Thesis Outline}

The thesis is structured into six chapters which may be summarised as follows:

\section{Chapter 1: Introduction}

This chapter provides an introduction to the research project and briefly describes its background. It justifies the need for the research and outlines the associated objectives and scope. Chapter 1 also provides a perspective of the issues related to the production of pharmaceutical products in industry, problems that can occur in the extraction of bio-products and an overview of reverse micelle extraction method.

\section{Chapter 2: Literature Review}

Chapter 2 reviews the research literature that surrounds the methods used in the production of pharmaceutical products and the advantages and disadvantages of the current methods. Fundamental aspects of reverse micelle systems, the formation of a reverse micelle and the solubilisation of bio-products in the reverse micelle system are discussed. Definition of a surfactant with explanations of its structures, categories and critical micelle concentration (CMC) is reviewed. The parameters that can be manipulated to achieve a high solubilisation of biomolecules for both forward and back-extraction processes are examined. The chapter also reviews the thermodynamic models proposed for the solubilisation process in a reverse micelle system, and describes the thermodynamic models used in the current study.

\section{Chapter 3: Experimental Procedures and Materials}

Chapter 3 describes the materials, equipment and experimental methods used in this research project, as well as the experimental methods of both forward and backward extraction. 


\section{Chapter 4: Results and Discussion - Forward Extraction}

Chapter 4 discusses the thermodynamic aspects of forward extraction in the light of both experimental results and modelling. The chapter presents experimental results for water content analysis, the effects of surfactant concentration, salt concentration, and $\mathrm{pH}$ on the extraction of penicillin $\mathrm{G}$ from the aqueous to the organic phase. Finally, Chapter 4 discusses the modelling of forward extraction.

\section{Chapter 5: Results and Discussion - Backward Extraction}

In a corresponding manner to Chapter 4 , Chapter 5 presents the experimental results and modelling applied to the reverse micellar process during backward extraction.

\section{Chapter 6: Conclusions}

This chapter covers the major findings, the conclusions of the study as well as the constraints, and provides recommendations for future work. 


\section{CHAPTER TWO \\ Literature Review and Concepts}

\subsection{Introduction}

This chapter starts by reviewing the relevant literature on separation methods of pharmaceutical products, including methods currently used in industry and those which are still under development. It then considers reverse micelle as an alternative separation method for bioproducts, followed by properties of a reverse micelle and surfactants. The ability of the reverse micelle to solubilise water and biomolecules is discussed. The parameters affecting the solubilisation of biomolecules into reverse micelle (forward extraction) and the release of biomolecules from a reverse micelle into an aqueous phase (backward extraction) are examined. Toward the end of the chapter, the thermodynamic models that describe the solubilisation of biomolecules into a reverse micelle are reviewed.

\subsection{Separation Methods in the Pharmaceutical Industry}

It has been discussed in many papers that the development of efficient methods for the separation of bioproducts from fermentation broths is crucial to advancements in biotechnology. Various forms of chromatography and electrophoresis are usefully applied in the purification of high value pharmaceuticals but are expensive and difficult to scale-up beyond laboratory sizes (Cascaval et al., 2001). Therefore, there is a significant need for efficient methods that can separate and concentrate pharmaceutical products continuously, with new cost-effective and simple separation techniques that can be scaled-up easily. 
For a wide range of chemical and biochemical products, the cost of separation and purification processes represents a significant proportion of the unit cost of production (Tessier et al., 2005, Soto et al., 2005), as well as the contribution of the process to the final cost being of $20.6 \%$ to $90 \%$ (Cascaval et al., 2001). It has been reported that fermentation based products are typically capital intensive because large and complex fermentors and extensive equipment for multi-step downstream processing are required to handle large volume fermentation broths with a low product concentration (Liu et al., 2004). As a consequence, liquid-liquid extraction has been widely used. Liquid-liquid extraction is the transfer of certain components from one phase to another when immiscible or partially soluble liquid phases are brought into contact with each other (Kilikian et al., 2000), according to their partition coefficient (Chimuka et al., 2004). The cost of liquid-liquid extraction is much lower than other more sophisticated bioseparations method such as liquid chromatography $(\mathrm{Gu}, 2000)$. The process design and its scale-up are easier than those for other separation techniques, and liquid-liquid extractions can minimise irreversible absorption losses and surface denaturation losses when compared to absorption methods (Gerson, 1988).

Unfortunately, since liquid-liquid extractions are infrequently used in the laboratory, they are often overlooked in process development and scale-up (Gerson, 1988). However, some attempts have been made to investigate the separation and purification processes that are based on liquid-liquid extraction techniques, especially the solvent extraction method as discussed thoroughly by Soto et al. (2005). Figure 2.1 shows a schematic diagram of the solvent extraction technique used in industry. Hatton (1985) asserted that an important step in the production of antibiotics is the recovery and concentration of the product from the fermentation broth in which it is produced. He further pointed out that the physicochemical properties which distinguish the desired solute from other fermentation products must be exploited in the selection of an appropriate separation technique for this step; liquid-liquid extraction has been successfully employed in the isolation of different bioproducts. The successful implementation of liquid-liquid extraction in the separation and concentration of bioproducts depends both on thermodynamic equilibrium constraints and on process considerations such as relative flow rates and the contacting patterns of the two phases. The partitioning behaviour of bioproducts 
can be influenced strongly by solution properties like $\mathrm{pH}$, ionic strength, antibiotic concentration, and can also be modified through the introduction of additives which interact with either the solute and/or the solvent to cause effective changes in the solute distribution between the phases.

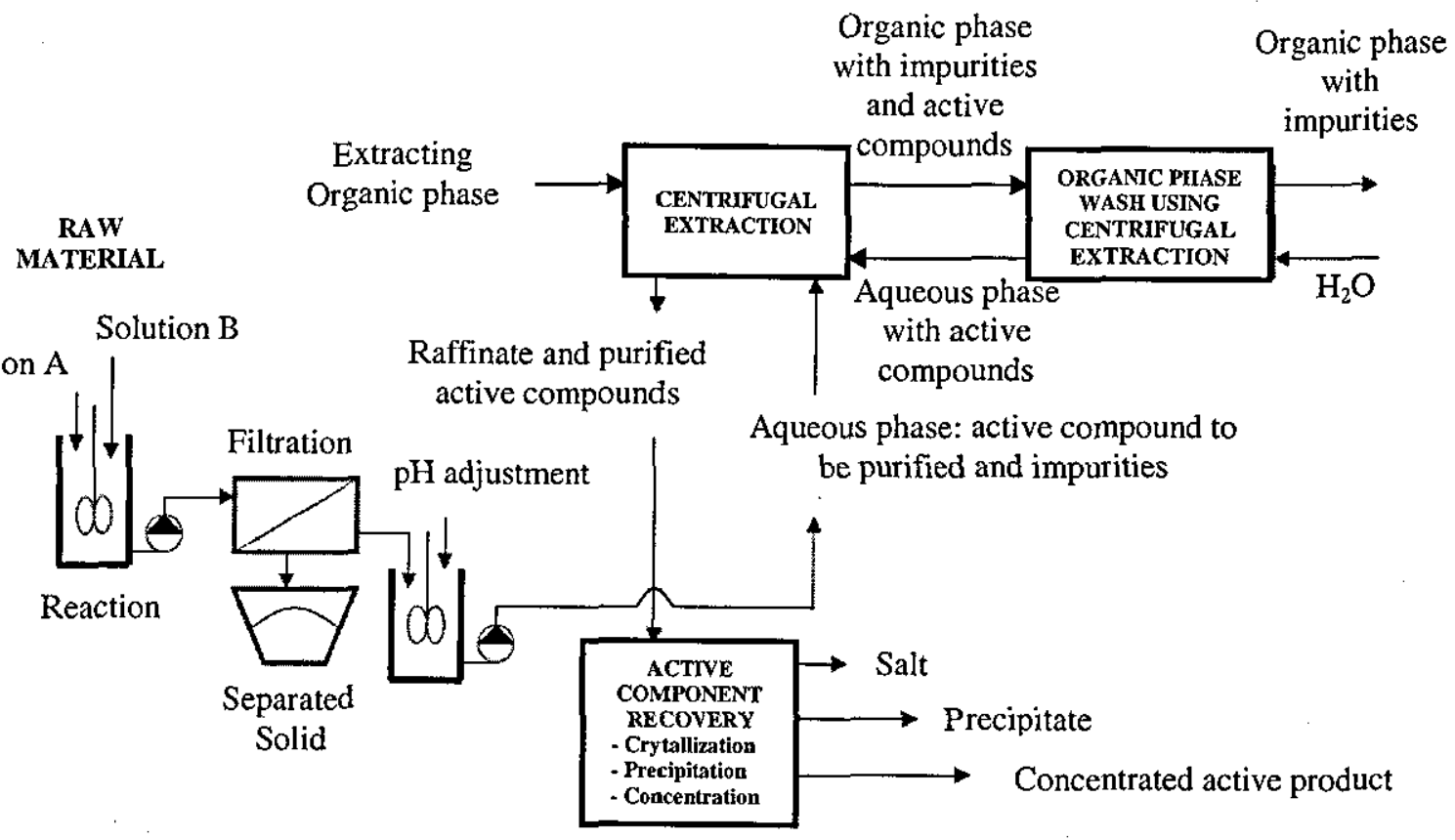

Figure 2.1: Schematic of the typical solvent extraction method used in industry. (Adapted from www.rousselet-robatel.com)

The effectiveness of the liquid-liquid extraction process also depends on the solvent selected for recovery of the solute from the fermentation broth (Liu et al., 2004). Popular solvents employed in the extraction of bioproducts are alcohols, ketones and acetates. These are all inexpensive and readily available, immiscible with aqueous solutions and exhibit the desirable physical properties of low viscosity and density which are significantly different from those of water. The selectivity of solvent for the product can usually be influenced by adjustment of the broth $\mathrm{pH}$, or by the introduction of other additives to the aqueous phase. Ordinary solvent extraction can be applied to large scale processes for separation of pharmaceutical products, e.g. antibiotics; however, its application is not always suitable because of denaturation in organic solvents (Yang et al., 1994; Liu et al., 2004; Tessier et al., 2005). Hu and Gulari (1996) argued that conventional organic solvent extraction for aminoglycoside molecules was inefficient because of their hydrophilic nature. They suggested that 
ion exchange and reverse micelle extraction are the appropriate alternative methods for the recovery of these products in industrial operations.

Alternative novel purification techniques such as reverse micelle systems have been developed, which may be used as a first step of the purification process, leading to higher recovery yields as reported by Aires-Barros and Cabral (1991). In the past decade, reverse micelle have attracted a lot of attention as a novel method for separating and purifying many biological products (Wolbert et al., 1989; Leser and Luisi, 1990; Ono et al., 1996), because reverse micelle provide a special microenvironment in an organic medium that make it possible to retain the structure of biomolecules that dominates their specific functions (Ono et al., 1996). A liquidliquid extraction with a reversed micellar phase has the capacity to solubilise specific biomolecules from dilute aqueous solutions such as fermentation and cell culture media (Kilikian et al., 2000). Reverse micelle is found to be a versatile tool for separating biomolecules and show a close similarity with liquid-liquid extraction since both are diphasic processes which consist of partitioning a targeted solute between an aqueous feed phase and an organic phase and then operating the back transfer to a second aqueous stripping phase (Rodrigues et al., 1999; Kilikian et al., 2000). Consequently, a potential biotechnology application of reverse micelle as an alternative for solvent extraction methods is one of the first steps in downstream processing (Regalado et al., 1996).

\subsubsection{Conventional Solvent Extraction}

Conventional solvent extraction is one of the most important techniques for recovering pharmaceutical products, e.g. antibiotics, from fermentation broths. The method has been used for over 100 years as an industrial unit operation (Grinbaum, 2003). The successful implementation of a solvent extraction process depends on the solvent selected for recovery of the solute from its fermentation broth (Hatton, 1985). The selection of solvents is crucial and must take account of their selectivity and capacity, price, availability, toxicity and process compatibility (Mat and Stuckey, 
1994). Popular solvents employed in antibiotic extractions are butanol, methyl isobutyl ketone and pentyl or butyl acetate.

Though the application of organic solvents to whole broth extraction of penicillin and other antibiotics is successful, there are a lot of potential disadvantages in using this extraction method which include:

1) The need to use a higher concentration of surfactants to achieve a reasonable phase separation. Also, for biochemical products such as penicillin, extracting solvents need to be added to get optimum product recovery (Verral, 1988).

2) A stable emulsion could be formed when denaturing of the antibiotics occurs during the solvent extraction process, such that a very efficient deemulsifier is required. The addition of demulsifiers increases the production' price and can cause environmental problems as emulsifiers cannot be recycled and discarded along with the aqueous solution (Yang et al., 1994; Nabais and Cardoso 1999; Yu et al., 2003; Tessier et al., 2005; Mathew and Juang, 2007).

3) For an unstable product such as penicillin $G$, a low temperature and expensive centrifugal extractor are required; however, there is still 10 to $15 \%$ loss of products (Yang and Cussler, 2000).

4) Solvents are very volatile compounds and can be evaporated easily, which leads to wastage of solvents (Yang et al., 1994, Nabais and Cardoso, 1999).

5) Some antibiotics such as cephalosporin are difficult to isolate by solvent extraction because of their amphoteric nature and high solubility in water (Ghosh et al., 1997).

6) The loss of solvents could occur because of only partial miscibility with water as a result of their fairly high dielectric permittivity values. Their ionic nature at high $\mathrm{pH}$ and instability at low $\mathrm{pH}$ increases the difficulties associated with solvent extraction of antibiotics (Ghosh et al., 1997). 


\subsubsection{Liquid Membrane Extraction}

Extraction using liquid membranes has been studied since the 1980's and is one of the most advantageous separation techniques present (Cascaval et al., 2001; Goto, 2006). The method entails transferring a solute between two aqueous phases at different $\mathrm{pH}$ values, which are separated by a solvent and a carrier layer. Figure 2.2 illustrates two types of liquid membrane.

a)

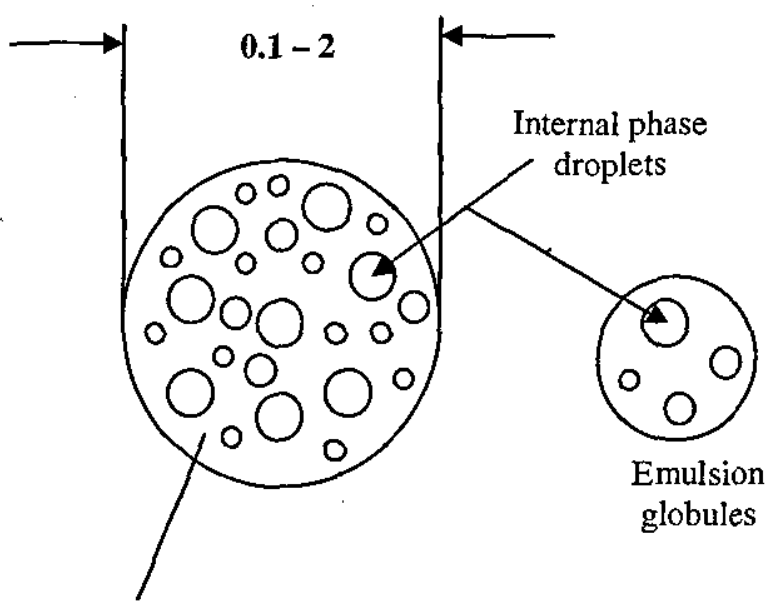

Oil membrane

bulk phase

b) Inlet stripping solution

Outlet stripping solution

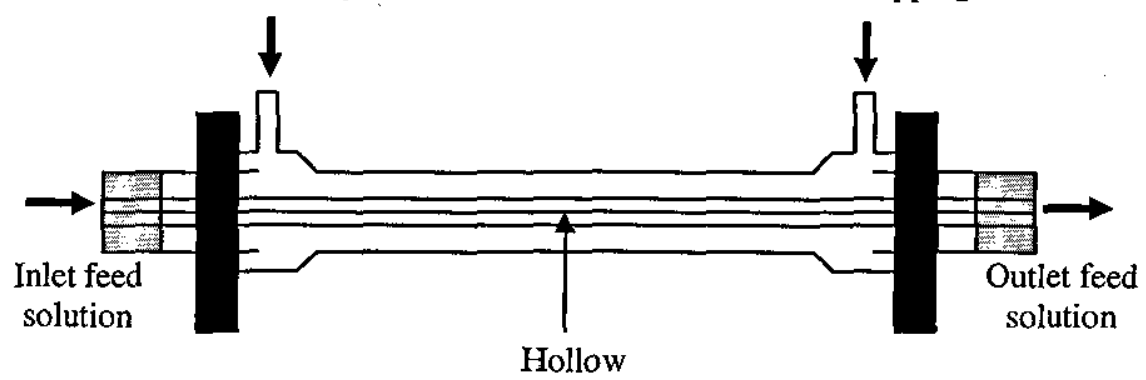

Figure 2.2: Liquid membranes: (a) emulsion liquid membrane, (b) supported liquid membrane.

A liquid membrane consistş of two immiscible phases namely oil membrane bulk phase and internal phase droplets. The later is also known as the continuous phase which contained emulsion globules as the continuous phase (Thien et al., 1986). Commonly, liquid membranes are obtained either by emulsification (emulsion liquid membrane, ELM) when their stability is poor, or by including the solvent in a 
hydrophobic porous polymer matrix (supported liquid membrane, SLM). Cascaval et al. (2001) carried out a comparison between extraction using liquid membranes and conventional liquid-liquid extraction. The claimed advantages are as follows:

1) The quantity of solvent used is small because of its continuous regeneration.

2) The loss of solvent is small during extraction process.

3) Provided the $\mathrm{pH}$ gradient between the two aqueous phases is maintained, there is a possibility of solute transport through liquid membranes that have been used for the separation of some biosynthetic products, namely carboxylic acids, amino acids and antibiotics.

Since its discovery two decades ago, the ELM has been studied for the separation of metal ions, amino acids, and carboxylic acids from dilute aqueous (e.g., Ho and $\mathrm{Li}$, 1992). ELM extraction does not encounter the equilibrium limitations of conventional solvent extraction processes and can offer high selectivity of separation of solutes from low concentration feeds (Ghosh et al., 1997). However, ELM also suffers from some major drawbacks which make the system difficult to operate commercially (Pellegrino and Noble, 1990):

1) The toxicity of the carrier and solvent should be carefully considered when designing an ELM system for the separation of antibiotics.

2) Stability of the membrane is needed to obtain an efficient initial mass transfer and this requirement always causes an increase in process costs.

A liquid membrane involving a microporous polymeric support membrane, the socalled SLM, can be more feasible for scale-up and adaptability for continuous operation (Ghosh et al., 1997). However, the system suffers from the drawbacks of high mass transfer resistance across the liquid membrane and low membrane stability. 


\subsubsection{Aqueous Two-Phase System}

Liquid-liquid extraction processes found in the chemical processing industry employ immiscible aqueous and organic phases to separate components, including those used successfully in the purification of antibiotics. However, many biologically active compounds are prone to damage by exposure to organic solvents. An alternative to organic-aqueous extraction processes is to use aqueous solutions of hydrophilic polymers, which can be induced to form two aqueous phases under certain conditions (Cascaval et al., 2001; Mathew and Juang, 2007). Aqueous two-phase systems (ATPS) are formed by two different polymers, the most widely used combination being dextran and polyethylene glycol, or polymer and a salt (Ghosh et al., 1997).

Aqueous two-phase systems have been used for the recovery of a variety of intracellular enzymes from disrupted cell broths, purification of interferon from mammalian cell cultures, affinity purification of enzymes and proteins and extractive bioconversion (Lee and Sandler, 1990). In an ATPS, a given molecule partitions between the two phases depending on its partition coefficient, defined as the ratio of the molecule concentration in the one phase to that in the other phase. The advantages of high water content, biocompatibility, low cost and space-time yield support the extended use of this technique (Rito-Palomares et al., 1996). On the other hand, ATPS has disadvantages as follows:

1) The cost of a polymer/polymer system, which typically consists of fractioned dextran as the bottom phase polymer, is too expensive to apply in large-scale applications (Ghosh et al., 1997).

2) The ionic strength of the salt used is high which severely influences the affinity partitioning of the biomolecules to be extracted (Ghosh et al., 1997).

3) The polluting effect of the phase components used. The cost of disposal is increased due to the presence of phosphate, which is one of the most difficult nutrients to be removed efficiently in waste treatment plants (Verral, 1988). 
4) A high centrifugal force is generally required to obtain an efficient separation of bio-products (Yang et al., 1994). The consumption of chemicals gets higher and increase the operational costs.

\subsubsection{Chromatography}

In chromatography, components of a solution are separated due to the partitioning of the components between a solid (stationary) phase and a fluid (mobile) phase. Partitioning occurs on the basis of charge, polarity, hydrophobicity, size or affinity. There are a number of different types of chromatography based on the interactions which occur between the solutes and the stationary phase (Verral, 1988). In spite of being relatively costly, chromatographic methods continue to be dominantly practiced on the preparative scale. Thin layer chromatography (TLC) and paper chromatography (PC), which offer good possibilities for physical separation, are applicable for analytical purposes only. High performance liquid chromatography (HPLC) offers the advantages of superior resolution and speed of operation and nearly all-new antibiotics are studied from development to clinical application by using HPLC. However, the use of HPLC is still limited to laboratory analysis and difficult to scale up for industrial purposes (Cascaval et al., 2001). Whilst chromatographic methods show promising results there are potential drawbacks. One of the disadvantages is that the use of these methods is considered as the high end of separation and purification.

\subsection{Fundamentals of Reverse Micelle}

Generally, most conventional extraction methods (as detailed in Section 2.2) have the potential to be utilised in the downstream processing of antibiotics and further research is being conducted to improve these methods for large-scale application. However, one method that has shown great potential is a system that utilises reverse micelle in the extraction of the required bioproduct. Carlson and Nagarajan (1992) discussed the applications in which reverse micelle systems may offer a promising 
alternative to chromatography for the large scale purification of proteins. In theory, this system is much easier to scale up than chromatographic separation processes and can allow for continuous separation processes for biomolecules (see Table 2.1).

Table 2.1: Features of separation methods in the pharmaceutical industry.

\begin{tabular}{|c|c|c|c|c|c|}
\hline & $\begin{array}{c}\text { Liquid } \\
\text { membrane } \\
\text { (Pellegrino } \\
\text { and Noble, } \\
\text { 1990; Ghosh } \text { et } \\
\text { al., 1997; } \\
\text { Cascaval et al., } \\
\text { 2000) }\end{array}$ & $\begin{array}{c}\text { Aqueous } \\
\text { two phase } \\
\text { system } \\
\text { (Verral, } \\
\text { 1988; Yang } \\
\text { et al., 1994) }\end{array}$ & $\begin{array}{l}\text { Chromatography } \\
\text { (Kinugasa et al., } \\
\text { 1991; Pires } \text { et al., } \\
\text { 1996; Ghosh } \text { et al., } \\
\text { 1997; Kyung and } \\
\text { Jee, 2004) }\end{array}$ & $\begin{array}{c}\text { Conventional } \\
\text { solvent extraction } \\
\text { (Kinugasa et al., } \\
\text { 1991; Pires } \text { et al., } \\
\text { 1996; Ghosh et al., } \\
\text { 1997; Nabais and } \\
\text { Cardoso, 1999; Liu et } \\
\text { al., 2001; Naoe } \text { et al., } \\
\text { 2004 }\end{array}$ & $\begin{array}{c}\text { Reverse micelle } \\
\text { extraction (Pires et } \\
\text { al., 1996; Ghosh } \text { et } \\
\text { al., 1997; Naoe et al., } \\
\text { 2004; Kyung and Jee, } \\
\text { 2004) }\end{array}$ \\
\hline Efficiency & Very good & Low & Very good & Good & Very good \\
\hline Yield /cycle & Can be poor & Can be high & Can be poor & $\begin{array}{c}\text { Very good, can be } \\
\text { low }\end{array}$ & Very good \\
\hline Selectivity & Very good & Can be good & Very good & Moderate & Good \\
\hline $\begin{array}{c}\text { Time } \\
\text { consumed }\end{array}$ & $\begin{array}{c}\text { No } \\
\text { information }\end{array}$ & $\begin{array}{c}\text { No } \\
\text { information }\end{array}$ & Long & Long & Short \\
\hline $\begin{array}{c}\text { Toxicity of } \\
\text { Solvent }\end{array}$ & High & High & $\begin{array}{c}\text { Information not } \\
\text { available }\end{array}$ & $\begin{array}{l}\text { High (because of } \\
\text { demulsifies) }\end{array}$ & No information \\
\hline $\begin{array}{l}\text { Denaturing } \\
\text { of product }\end{array}$ & $\begin{array}{c}\text { No } \\
\text { information }\end{array}$ & $\begin{array}{c}\text { Low } \\
\text { possibility }\end{array}$ & Low & High possibility & Very low \\
\hline $\begin{array}{c}\text { Wastage of } \\
\text { solvent }\end{array}$ & Very low & Very low & Low & High & No information \\
\hline Cost & Very high & Very high & Very high & Can be high & Can be low \\
\hline $\begin{array}{l}\text { Scale-up } \\
\text { potential }\end{array}$ & $\begin{array}{c}\text { Moderate } \\
\text { (low stability) }\end{array}$ & Can be good & $\begin{array}{l}\text { Poor (limited to } \\
\text { laboratory) }\end{array}$ & Very good & Very good \\
\hline $\begin{array}{l}\text { Method } \\
\text { established }\end{array}$ & No & No & No & Yes & No \\
\hline
\end{tabular}

Reverse micelle is an alternative method to conventional separation and purification procedures due to their potential for large scale use (Ono et al., 1996; Naoe et al., 2004; Shen and $\mathrm{Yu}, 2007$; Moore and Palepu, 2007), their potential application to continuous separation of biological substances (Zhang et al., 1999; Hong and Kuboi, 1999; Naoe et al., 2002; Noh and Imm, 2005; Juang et al., 2006; Majumdar and Mahapatra, 2007), the process similarities to liquid-liquid extraction (Naoe et al., 2004; Hasmann et al., 2007), and easy scale-up (Chang et al., 1997; Rodrigues et al., 1999; Kilikian et al., 2000; Mathew and Juang, 2007). Nishiki et al. (1998) showed that reverse micelle extraction is able to recover proteins with little denaturation because proteins can be hosted in a solubilised aqueous phase which is shielded from the organic solvent by surfactant molecules; solubilisation inside reverse micelle does not damage the native protein structure (Yu et al., 2003; Hetch and Peled, 
2006). Therefore, in an ordinary liquid-liquid extraction, although it has been difficult to extract large bioactive molecules such as proteins, the limitation can be overcome by utilising a nanostructural molecular assembly like reverse micelle (Aires-Barros and Cabral, 1991; Ono et al., 1996).

Reverse micelle are nanometre aggregates of surfactant molecules in a polar solvents (see Figure 2.3). They can be regarded as very simple bioaggregates (Lee et al., 2001; Freda et al., 2002; Havre, 2002; Debnath et al., 2007), dispersed in a continuous organic solvent medium with the help of a surfactant (Castagnola and Dutta, 2000; Kinugasa et al., 2003; Paul and Mitra, 2006).

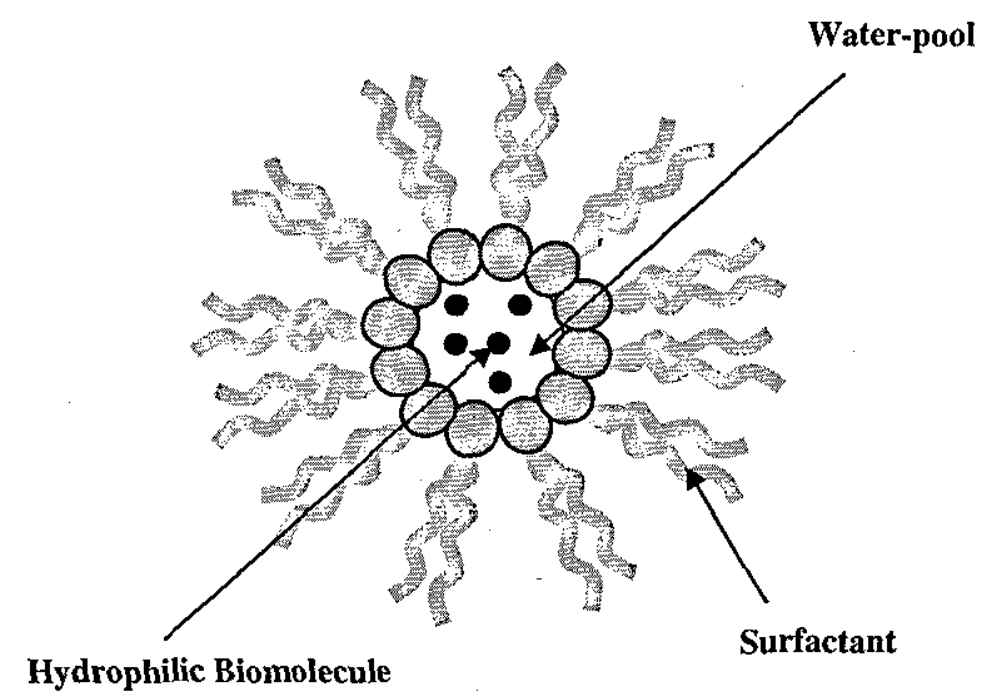

Figure 2.3: Schematic representation of a reverse micelle (adapted from Vyve and Renken, 1999).

Surface active reagents, better known as surfactants, are molecules made up of hydrophobic and hydrophilic structures that are able to modify the interfacial properties of the liquid (non-aqueous or aqueous) in which they are present (Yang, 2001). The hydrophilic and hydrophobic regions are illustrated in Figure 2.4.

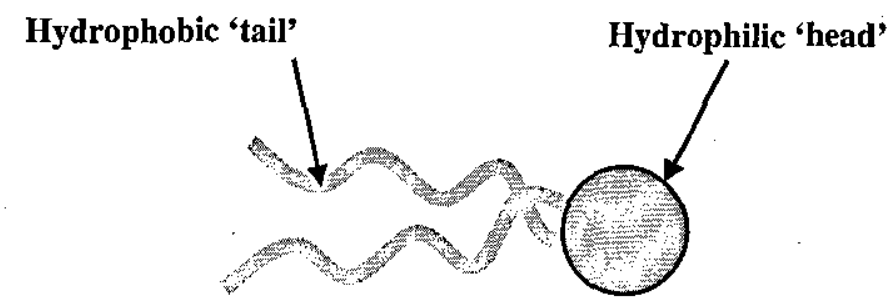

Figure 2.4: Structure of an AOT (adapted from Yang, 2001). 
Working in reverse micelle phases presents many advantages (Vyve and Renken, 1999). The reverse micelle can dissolve molecules which are normally not soluble in the bulk solvent. Also, due to the very small size of a micelle, there is an enormous increase of the interfacial area between the continuous and the dispersed pseudophases in comparison to a mechanically stirred system without surfactants. Mathew and Juang (2007) summarised the main advantages of using reverse micelle as the alternative to conventional extraction method as follows:

1) Solublisation behaviour of biomolecules in reverse micelle can be regulated by varying the size and shape of the reverse micelle.

2) Selective partitioning of biomolecules can be achieved in the extraction based on the hydrophobic nature of proteins due to the fact that reverse micelle provide the hydrophobic and hydrophilic environments to solutes simultaneously.

3) Recovery of biomolecules from the reverse micelle can be easily facilitated by exploiting the de-assembling nature of reverse micelle in the aqueous media. Moreover, the surfactants can be separated from the system by filtration and recycled.

Reverse micelle extraction of biomolecule products is a relatively new discovery. Kilikian et al. (2000) suggested that a growing number of studies on reverse micelle techniques clearly demonstrate the scientific interest in reversed micelles in the separation of biotechnology products. A reverse micelle is a droplet containing an inner core of water in an aqueous solution (Figure 2.3) where size may vary in the order of 50 to $100 \AA$ (Wang et al., 1995). Reverse micelle is based on a water phase and a hydrocarbon phase, and is a thermodynamically stable mixture of water, oil and surfactants where the water regions are separated from oil by a monolayer of surfactants (Hong and Kuboi, 1999; Goto et al., 1998; Pandit and Basu, 2002; Bong et al., 2004). Baier (1999) found that reverse micelle is a group of individual surfactant molecules arranged with the hydrophilic ends concentrated together and surrounded by the hydrophobic ends. The aqueous phase is stabilized in a polar environment with the presence of surfactant at the interface. In this way small hydrophilic regions are created within the organic phase. When a reverse micelle 
hydrocarbon phase is contacted with an aqueous phase containing a bio-product, the bio-product may be captured into the micelle.

To date, the reverse micelle system has been used for the separation of proteins (Goklen and Hatton, 1987; Aires-Barros and Cabral, 1991; Brandani et al., 1996; Pires et al., 1996; Su and Lee, 1999; Kinugasa et al., 2003; Bong et al., 2004; Jun et al., 2004; Noh and Imm, 2005; Hecth and Peled, 2006; Shen and $\mathrm{Yu}, 2007$ ), peptides (Kishi and Furusaki, 1992), amino acids (Leodidis and Hatton, 1989; Furusaki and Kishi, 1992; Adachi et al., 2000; Dövyap et al., 2006), bovine serum albumin (BSA) (Zhang et al., 1999), enzymes (Moreno-Hagelsieb et al., 1999; Liu et al., 2004; Bong et al., 2004; Debnath et al., 2007) and metals (Nakashio et al., 1992; Ismael and Tondre, 1992; Zhang et al., 2006; Majumdar and Mahapatra, 2007) as well as in a bioreactor to carry out biotransformation (Kamiya et al., 1995). Thus, reverse micelle has been demonstrated to be capable of hosting large quantities of biomolecules without causing denaturation by adjusting operational parameters (Hatton, 1985; Paradkar and Dordick, 1994; Goto et al., 1998; Cardoso et al., 1999; Shen and $\mathrm{Yu}, 2007)$.

\subsection{Formation of Reverse Micelle}

Figure 2.5 shows the comparison between normal micelle and reverse micelle. Water plays a decisive role in the formation of reverse micelle. The surfactant molecules aggregate at interfaces forming a middle phase in equilibrium with an excess organic phase and an excess aqueous phase. The transition from micelle to reverse-micelle system, as described by Esalah (1997), is generally driven by a decrease of the surfactant hydrophilicity. Increasing the salinity of the aqueous constituent by adding a medium-chained alcohol (co-surfactant) decreases the surfactant hydrophilicity.

The structures of biomacromolecules are very sensitive to the organic solvent involved (Chang et al., 2000). Using reverse micelle systems can therefore create a suitable environment for solubilising the biomolecule solutes into the organic solvent 
region. Reverse micelle can be formed in an organic phase by a contact method or a titration method (Rabie et al., 1997). These two methods have been found to be significantly different with respect to their solubilisation capacities for water and different solutes such as salts, amino acids, proteins, antibiotics, etc. Rabie (1997) found that the titration method had been used widely to form reverse micelle as injection fluids for a number of tertiary oil recovery processes and also as microreactors in which guest molecules could be brought to react, often showing reactivity that differed markedly from that observed in pure solvents. The titration method on the other hand is a process where an aqueous solution is titrated into an organic solvent containing a surfactant and a co-surfactant. The maximum mass of aqueous solution that can be solubilised is determined using the appearance of permanent turbidity. In this method, there is no excess aqueous phase in equilibrium with the organic phase containing the reverse micelle. Since there is no excess aqueous phase present, the composition of the water pool is identical to the composition of the titrant. The use of an electrolyte is not required in the titration method but, if present in the titrant, its concentration affects the maximum water solubilisation.

(a)

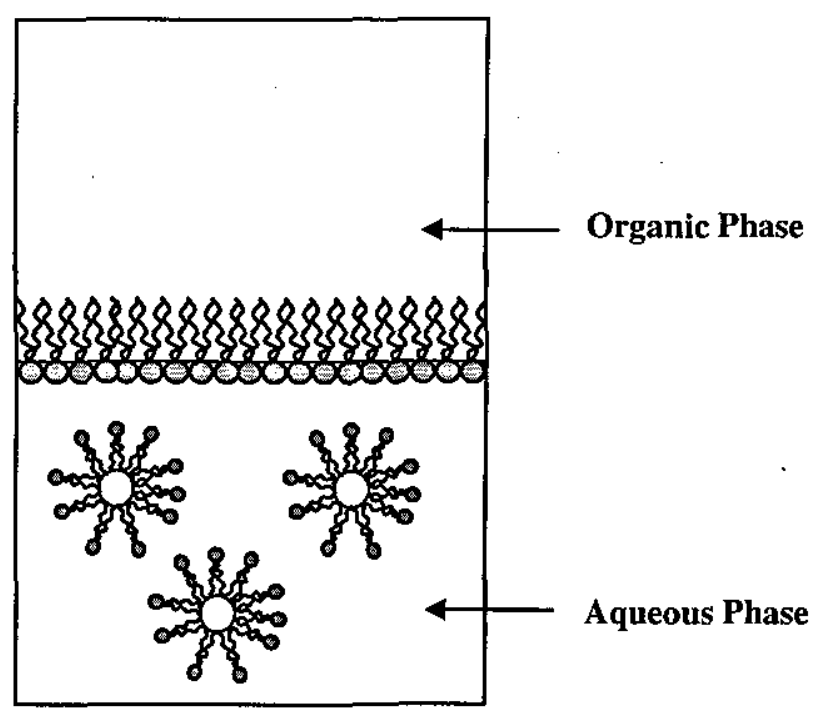

(b)

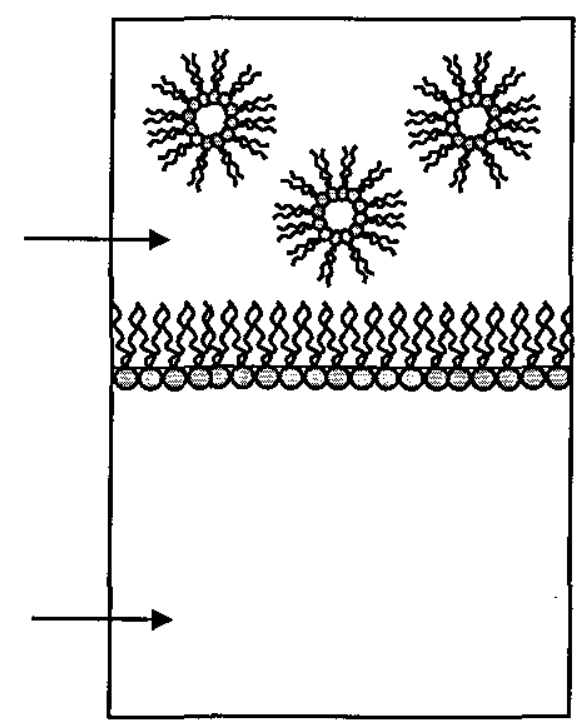

Figure 2.5: Aggregation of surfactants; (a) micelle, and (b) reverse micelle (adapted from Esalah, 1997). 
The contact method is a process where a bulk excess aqueous phase is contacted with an organic solvent containing the surfactant and co-surfactant and identical to the basic step used in the extraction of solutes from water into a reverse micelle organic phase. The excess aqueous phase must contain an electrolyte to prevent the transfer of the surfactant from the organic phase to the aqueous phase. After mixing and settling, the organic phase equilibrates with an excess aqueous phase. The amount of water solubilised is obtained by measuring the water content of the organic phase, usually by a Karl Fischer titration. The composition of the water pool is determined by the exchange equilibrium of ions and solutes between the phases.

\subsection{Surfactants}

Surfactants and soaps comprise a large category of amphiphilic, surface active compounds used in industrial applications, household cleansers and scientific research. Surfactant applications are present in almost every chemical industry and can be found in every cleaning or conditioning product and in the oil industry (Yang, 2001). Crude production of these materials is easily accomplished by mixing animal fats with wood ash, followed by saponification to create free fatty acids. Toerne (2002) also mentioned that the production of surfactants is thought to have been taking place for more than 2500 years, and that the industrial synthesis of soaps in recent times has exceeded $\$ 10$ billion per year in the United States alone. Moreover, the investigation and utilization of organized assemblies of surfactant molecules is one of the most active areas of colloid chemistry and it is well known that among the various kinds of assemblies, reverse micelle have drawn considerable attention in recent years. The surfactant plays an important role in forming a spherical shell surrounding the micelle (Kilikian et al., 2000).

By way of example, Pandit and Basu (2002) studied the comparison of dye removal from the aqueous phase with and without using surfactant. They found that the dye removal efficiency without surfactants was very low compared to the percentage removal when using surfactant. In the absence of surfactant, the removal of methyl orange from water to amyl alcohol follows a conventional solvent extraction process 
and yields only $7 \%$ extraction of methyl orange. The percentage removal of methyl orange increases drastically up to $80 \%$ in the presence of the HTAB surfactants due to encapsulation of the dye in the reverse micelle formed by the alcohol.

\subsubsection{Surfactant Structure}

Most surfactants have a long hydrocarbon tail that can be linear or branched and interact only very weakly with the water molecules in an aqueous environment; hence the chain is called a hydrophobic 'tail' (see also Figure 2.4). The hydrophilic 'head' is a relatively small ionic or polar group that interacts strongly with the water via dipole-dipole or ion-dipole interactions. As a surface-active reagent, the surfactant molecule is capable of changing the interfacial free energy, which is the internal energy of an interface. The molecules tend to aggregate at the interface between an aqueous phase and another phase of the system where they then reduce the surface or interfacial tension (Esalah, 1996).

The intermolecular forces that keep the aggregates together arise from weak van der Waals forces, hydrophobic interactions, screened electrostatic interactions and hydrogen bonding (Chang et al., 2000). At relatively high concentrations in water, aqueous soluble surfactants self associate to form micelles which are aggregates characterized by a non-polar core formed by hydrocarbon groups and hydrophilic groups facing outwards towards the water. When this aqueous solution is contacted with an organic phase, the surfactant molecules may migrate to the organic phase and aggregate, thus forming reverse micelle.

\subsubsection{Categories of Surfactant}

Surfactants can be divided into four categories: anionic, cationic, nonionic and zwitterionic that contain both anionic and cationic charges on the same molecule (Rabie and Vera, 1996; Chang et al., 2000). Surfactants tend to migrate to surfaces and alter free energy states or interfacial tensions (free energy per unit area), which 
can result in the formation of emulsions and thus greatly increase the interfacial area between immiscible liquids like oil and water. Molecules that possess both the hydrophilic and hydrophobic parts are termed amphiphiles or amphiphatics.

In solvents with different polarity, an orientation can be seen whereby the surfactant molecules align to shield the hydrophobic heads from the polar solvent. The nonpolar tail of surfactant molecules comes into contact with the organic phase and the polar heads sequester together, forming a vesicle that encloses the water pool inside the cavity (Chang et al., 2000). Havre (2002) postulated that hydrophobic parts of the molecules are directed into the centre of the micelles, while the water-soluble, hydrophilic part is directed towards the water phase. The aggregation process is driven by an increase in entropy. When a hydrocarbon chain is in contact with water, the water molecules close to the chain order themselves thus leading to a reduction in the entropy compared to the bulk of the water. The entropy increases if the hydrocarbon chains are removed from contact with the water, into the micelle.

Ionic reverse micelle are more frequently investigated compared to cationic system. For example, Sodium di(2-ethylhexyl)sulfosuccinate (AOT), an anionic surfactant, has been used widely because of its ability to form reverse micelle containing large amounts of water (Rabie et al., 1997; Moore and Palepu, 2007). Extraction using heptane as the oil can produce a maximum solubility of water of about 70 molecules per AOT molecule at room temperature (Bardez et al., 1996). Adachi et al. (2000) demonstrated that reversed micelle composed of non-ionic surfactants interact mildly with proteins, resulting in low denaturing of proteins and has no capability of extracting water soluble proteins because of the lack of strong interactions between the reverse micelle and proteins.

Most studies on reverse micelle extraction have employed an anionic surfactant such as AOT (Goklen and Hatton, 1987; Dungan et al., 1991; Naoe et al., 1999; Castagnola and Dutta, 2000; Franqueville et al., 2003; Shin et al., 2003; Zhang et al., 2006; Majumdar and Mahapatra, 2007; Mathew and Juang, 2007,) which forms stable reverse micelle even with high water contents, in a wide variety of solvents (Freda et al., 2002). Anionic molecules spontaneously form spherical aggregates that have a narrow size distribution and a negatively charged polar head group, 
thereby enabling the positively charged solute to be extracted into the reverse micelle (Naoe et al., 1999). Moreover, strong electrostatic interactions between the protein and the polar head groups of the surfactant permits stable and speedy extraction that is responsive to the operational parameters (Goklen and Hatton, 1987).

As an anionic surfactant, AOT has the capacity to form large micelles in apolar solvents without a co-surfactant (Andrews, 1993; Kilikian et al., 2000; Paul and Mitra, 2006). Hossain et al. (1999) suggested that the motivation to use an anionic surfactant AOT in their studies was the large amount of published data on the physico-chemical properties of AOT reverse micelle and the ease of reverse micelle formation. Ionic surfactants such as AOT can spontaneously form reverse micelle with a micro water pool in an apolar medium thereby enabling the charged biomolecule to be solubilised into the reverse micelle (Naoe et al., 1999). Yonker et al. (2003) used AOT in their study because it is well known that the aqueous core regions of the AOT micelle are capable of dissolving a broad range of highly polar, ionic and/or high molecular weight species.

\subsubsection{Critical Micelle Concentration (CMC)}

Critical micelle concentration (CMC) is the minimum surfactant concentration needed for micelle to form and represents the concentration of free or unaggregated surfactant molecules in equilibrium with the micelle (Frense et al., 1995; Kilikian et al., 2000; Michaels et al., 2007). The CMC is a characteristic of the system and dependent on temperature, pressure, solvent, and chemical structure of the surfactant (Rabie and Vera, 1996). Therefore, if the concentration of the surfactant is below the CMC value, then surfactant molecules can only exist as free molecules in the solution without the formation of micelles.

The CMC is often determined by measuring the surface tension at different concentrations of surfactant (Havre, 2002). Upon addition of a surfactant to an aqueous solution, the surface tension decreases until the CMC is reached. Above this concentration, surfactant that is added will form micelles and the concentration of 
monomeric surfactant will remain practically constant, resulting in a constant surface tension. The CMC can also be determined by measuring other physicochemical properties that change at the $\mathrm{CMC}$ such as electric conductivity, refractive index, osmotic pressure, turbidity, $\mathrm{x}$-ray diffraction, or viscosity. The CMC value is. obtained by plotting the values of one (or more) of these properties against the surfactant concentration (Figure 2.6).

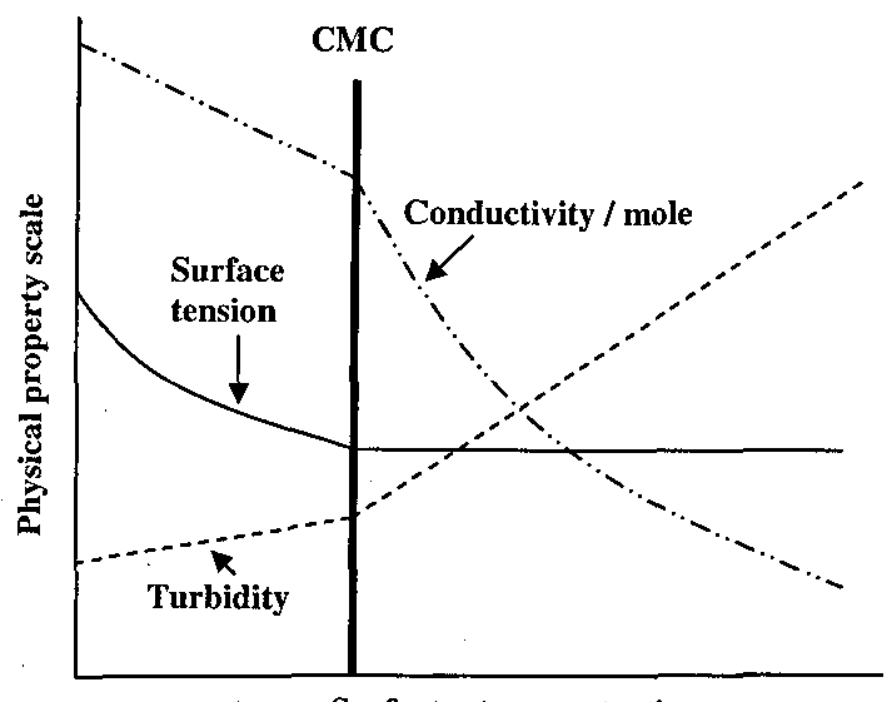

Surfactant concentration

Figure 2.6: Effect of surfactant concentration on several solution properties, and the consequence of micelle formation on these properties.

The CMC is denoted by a sharp change in the trends of these physical parameters. Below this point, surfactants are thought to exist predominately as monomers, while a population of free monomers at a concentration near the CMC remains outside the aggregates (Toerne, 2002). The CMC is believed to be the point at which solution interfaces are saturated with surfactant solubilisation into the bulk solvent through the creation of micelle (Figure 2.7). Micelle formation solubilises the surfactant into the bulk solution where its surface activity is lowered, resulting in small surface tension changes above the $\mathrm{CMC}$. 


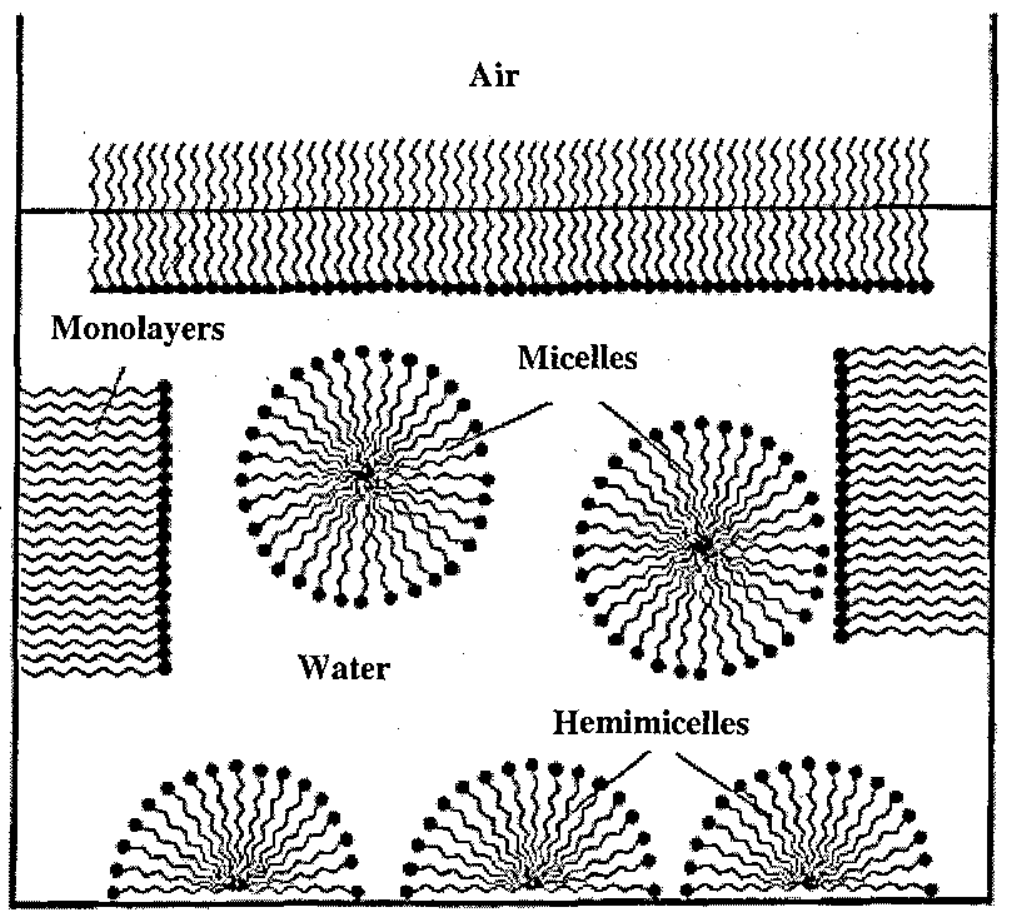

Figure 2.7: Representation of a few surfactant structures that are thought to form at concentrations greater than CMC (adapted from Toerne, 2002).

Addition of an ionic surfactant to water leads to a reduction in the molar conductivity up to $\mathrm{CMC}$, where the decrease becomes more dramatic. This is due to the decreased electrophoretic mobility of micelle. Moreover, the surface organization of surfactants exhibits interesting characteristics at concentrations above and below the CMC. Studies of various hydrophilic and hydrophobic surfaces at widely varying surfactant concentrations have revealed the existence of hemimicelle structures, monolayers or hexagonal packing of rods absorbed into solution interfaces (Toerne, 2002).

\subsection{Solubilisation in Reverse Micelle}

When an organic phase containing ionic surfactants is contacted with an aqueous phase containing salts, thermodynamically stable reverse micelle may form. The reverse micelle can also solubilise large amounts of water, forming isotropic solutions called water-in-oil-microemulsions (Rabie et al., 1997). The formation of reverse micelle permits the solubilisation of different biomolecules and salts in an 
organic phase. The term solubilisation, which is a key concept in the study, was originally used for aqueous micelle solutions but was generalised to include reverse micelle in apolar solvents.

It is well known that the extraction of a biomolecule is governed by the hydrophobic interaction between the biomolecule and the surfactant headgroup. For example, amino acids with hydrophobic side chains, such as tryptophan, are mainly incorporated in the interfacial zone of the reversed micelles while the hydrophilic amino acids are taken up in the micro water pool in the reversed micelle (Adachi $e t$ al., 1991). Leodidis and Hatton (1989) pointed out the importance of hydrophobic effects as a driving force for interfacial solubilisation by correlating hydrophobicity with the free energy of transfer of a large number of amino acids from water to the surfactant interfaces. Reverse micelle extraction of oligopeptides was investigated by Kishi and Furusaki (1992) who found that the partition coefficient of the peptides increased with an increase in the hydrophobicity of the peptide. It is also likely that proteins with surface hydrophobic regions exhibit an anomalous behavior, being extracted to the reversed micelle organic phase at unfavorable values of $\mathrm{pH}$ or high salt concentration as observed by Aires-Barros and Cabral (1991).

Goto et al. (1998) suggested that a key factor in reverse micelle extraction is the hydrophobicity of the intermediate surfactant-protein complex and not the characteristics of the reversed micelles. When proteins are interacted with a high hydrophobicity surfactant such as dioleylphosphoric acid (DOLPA), the solubilisation of the proteins by the organic phase is facilitated. The low hydrophobicity interfacial complex of protein and DLIPA (dilinoleyl phosphoric acid) is not extracted into a reverse micelle organic phase. Leodidis and Hatton (1990) found that the hydrophobic amino acids were taken up both in the water pool and in the interfacial zone, but the hydrophilic amino acids were taken up only in the water pool. Yu et al. (2003) shared the similar view when they revealed hydrophobic interactions between the lipase and the surfactant and/or organic solvent are also important in the extraction of the lipase. This suggests that in the process of AOT reverse micelle extracting yeast-lipase, electrostatic and hydrophobic mechanisms exists simultaneously. Therefore, the hydrophobicity of the intermediate complex is a key factor in determining the extraction efficiency (Ono et al., 1996). 
A key parameter in all reverse micelle applications is the amount of water which the reverse micelle solubilise in the organic phase (Wang et al., 1994; Zhao et al., 2007). The quantity of water that can be solubilised is expressed as $W_{o}$, the molar ratio of water to surfactant $\left(\left[\mathrm{H}_{2} \mathrm{O}\right] /[S]\right)$. Factors influencing water solubilisation are concentration and structure of surfactant, solvent, salt, biomolecule, temperature and pressure (Kinugasa et al., 1991; Ichikawa et al., 1992; Lye, 1993; Frense et al., 1995; Naoe et al., 1999; Michaels et al., 2007). Many researchers have found that the water content of the micellar organic phase is a significant parameter affecting biomolecule extraction (Andrews, 1993; Imai et al., 1996; Kilikian et al., 2000; Naoe et al., 2002; Nagayama et al., 1999; Wang et al., 2007). This parameter has also been found to determine the structure and size of reverse micelle; the size increases proportionally with $W_{o}$, (Rogrigues et al., 1999; Xun et al., 2001; Naoe et al., 2002). Perez-Casas et al. (1999) asserted that when small amounts of water are present, the reverse micelle aggregates formed in solution, whereas the dispersions formed when larger quantities of water are present should be termed microemulsions.

In AOT/isooctane systems, for instance, the micelle is spherical, nanometre sized particles (with diameters ranging from 10 to $200 \AA$ ) which are thermodynamically stable (Andrews, 1993; Kilikian et al., 2000). Lye (1993) asserted that for the AOT reverse micelle system, below $W_{o}=6-10$ all the solubilised water is bound to the interface as water of hydration for the sulfonate head group. He also concluded that this water has restricted mobility, decreased hydrogen bonding, depressed freezing point, decreased dielectric constant and increased microviscosity. At low water content, all water is accommodated in the interior of the reverse micelle, and the system is monophasic, whilst at higher water content two phases arise (Frense et al., 1995). Many reserchers agreed that with an increase of surfactant concentration, the micellar size does not change for many surfactants, especially for AOT (Zulauf and Eicke, 1970).

There are different views regarding the amount of water content in systems with (i.e. biomolecule-containing system) or without biomolecules (biomolecule-free system). Ichikawa et al. (1992) found that water solubilisation in a 'protein-containing 
system' was larger than in a 'protein-free system'. The difference between the two systems could have been induced by the rather small mass of protein extracted under the given experimental conditions. In contrast, Ono et al. (1996) pointed that the water content in a 'protein-free system' is always higher than that in the presence of protein. This result suggests that water never accompanies the protein molecules when it is dissolved into reversed micelle. However, Kinugasa et al. (1991) argued that there is no significant difference in the water content of reverse micellar solutions with and without the proteins.

\subsection{Biomolecule Partitioning in Reverse Micelle}

Selective extraction of biomolecules into reverse micelle can be achieved by manipulating the parameters of the system both in the reverse micelle and in the aqueous phase (Cardoso et al., 1999; Juang et al., 2006; Michaels et al., 2007). By choosing the right conditions ( $\mathrm{pH}$, temperature, ionic strength, surfactant type, etc.), molecules can be transferred to the reverse micelle due to the electrostatic interactions between the charged antibiotics and the polar heads of the surfactant or by hydrophobic interactions with the hydrophobic alkyl chains of the surfactant (Pessoa and Vitolo, 1998). Under certain conditions, the reverse micelle contained in organic solvent can exist in equilibrium with a bulk aqueous phase and can be used to extract polar materials from the aqueous phase (Hatton, 1985). To understand the thermodynamics of the extraction process, it is important to understand the factors affecting the extraction of pharmaceutical molecules.

Extraction is dependent upon many factors which are directly related to the interaction between properties inherent to the system itself and those pertaining to the biomolecules (Andrews, 1993). Fundamental studies of these factors are necessary to establish correlations between the physico-chemical properties of the molecules and the reverse micelle system. Goto et al. (1995) suggested that the extraction of solutes from an aqueous phase into the reverse micelle should be done by varying the experimental conditions such as $\mathrm{pH}$, ionic strength, surfactant concentration, etc. Besides, the extraction of antibiotics can also be influenced 
strongly by the solute concentration and solvent used (Hatton, 1985). The effect of these factors and the thermodynamic partitioning of antibiotics is discussed in Sections $2.7 .4,2.7 .5$ and 2.7 .8 respectively.

When using an anionic reverse micellar system for protein extraction, the distribution of proteins between the micellar organic phase and the aqueous phase is largely determined by the conditions in the aqueous bulk phase and the organic phase, e.g. the $\mathrm{pH}$ (Pires et al., 1996; Goto et al., 1998; Goto et al., 1999; Adachi et al., 2000; Xun et al., 2001; Jun et al., 2004; Noh and Imm, 2005), ionic strength (Pires et al., 1996; Goto et al., 1998; Goto et al., 1999; Jun et al., 2004; Noh and Imm, 2005), salt type (Pires et al., 1996), salt concentration (Verral, 1988; Frense et al., 1995; Kilikian et al., 2000), surfactant type (Goto et al., 1998; Goto et al., 1999), the presence of co-surfactant (Pires et al., 1995; Jun et al., 2004), and solvent type (Pires et al., 1995). However, $\mathrm{pH}$, ionic strength (Goklen and Hatton, 1987) and salt type (Pires et al., 1996) are the most dominant factors for the reverse micellar extraction .

\subsubsection{Aqueous Phase pH}

Reverse micelle systems have been studied extensively for the extraction of biomolecules, such as proteins and antibiotics, from aqueous solutions where $\mathrm{pH}$ is a major factor in extraction (Rabie and Vera, 1996). Chen et al. (1999) studied the effect of $\mathrm{pH}$ on the extraction of dipeptides and discovered that the synthesis of dipeptides was reflected in two respects:

1) $\mathrm{pH}$ can alter the transfer of the charged dipeptide product from the water pools of the reverse micelle to the bulk organic solvent

2) $\mathrm{pH}$ plays an important role in the stability of the reverse micelle constructed by the chosen anionic AOT surfactant

The transfer efficiency of biomoelcules into reverse micelle is strongly dependent on $\mathrm{pH}$. Su and Lee (1999) proposed that the low $\mathrm{pH}$ of aqueous feed solutions would 
give the optimum extraction of solutes into a reverse micelle phase. Moreover, the $\mathrm{pH}$ would also cause the solubilisation of the solute into the reverse micelle because of the attractive electrostatic and hydrophobic interactions between the charged surfactant head group and the solute in question. Hu and Gulari (1996) found that the transfer efficiency is strongly dependent on the $\mathrm{pH}$. The study concerned the extraction of amino glycoside antibiotics using an AOT reverse micelle system. For this particular study it was found that in the $\mathrm{pH}$ range of $8.5-11$ the transfer efficiency sharply decreased with $\mathrm{pH}$. The decline of the transfer efficiency with $\mathrm{pH}$ indicates that the forward extraction is a result of the attractive electrostatic interaction between the antibiotic molecules and the charged surfactant head group. As a result, as the $\mathrm{pH}$ increases, so the positive charge of the amino glycoside molecules decreases and the magnitude of the attractive interaction diminishes to decrease the transfer efficiency. The same phenomenon was also noticed for protein solubilization in reverse micelle (Leser and Luisi, 1990; Dekker et al., 1990; Lye et al., 1995).

The $\mathrm{pH}$ of the aqueous solution affects protein transfer into the reversed micellar phase because the $\mathrm{pH}$ determines the net charge of the proteins by nullifying the positive or negative charges of the molecule surface. The $\mathrm{pH}$ must be at a level that generates a protein net charge opposite in sign to the surfactant headgroup, so that there is an attraction between the protein surface and the polar headgroups on the internal surface of the reversed micelle (Kilikian et al., 2000). This difference in charge is the driving force for the process. The $\mathrm{pH}$ dependence means that protein transfer is governed by the electrostatic interaction between the proteins and the surfactant head groups (Ono et al., 1996, Yu et al., 2003). Therefore, only at pHs below the isoelectric point (pI) of the protein should the protein be extracted with anionic surfactants (Ono et al., 1996). At $\mathrm{pHs}$ above the pI, extraction into the reverse micellar phase would be inhibited due to unfavourable electric repulsion. Yu et al. (2003) revealed that the lipase could be completely solubilised into the reversed micellar solution at $\mathrm{pH}<\mathrm{pI}$, because under this condition the yeast-lipase was positively charged and hence attracted by the anionic AOT surfactant headgroups.

For $\mathrm{pH}>\mathrm{pI}$, the negatively charged protein is repelled by the negatively charged head of the amphiphilic AOT molecule (Hu and Gulari, 1996). In general, proteins 
are only transferred to a reverse micellar phase at $\mathrm{pHs}$ at which their net charge is opposite to that of the surfactant headgroups because solubilisation is usually steered by electrostatic interactions between the protein molecule and the surfactant headgroup.

\subsubsection{Salt Concentration and Type}

Addition of salt can make protein release easier at some concentrations (Brandani et al., 1996; Yu et al., 2003), however, many publications have demonstrated that the protein transfer would decrease with increasing salt concentration of the aqueous phase (Kinugasa et al., 1991; Ichikawa et al., 1992; Hu and Gulari, 1996; Regalado et al., 1996; Goto et al., 1999; Zhang et al., 1999; Kilikian et al., 2000; Kinugasa et al., 2003; Yu et al., 2003). The primary effect of salt is in shielding the electrostatic interaction between the micellar wall and protein ( $\mathrm{Hu}$ and Gulari, 1996). At high salt concentration, the interaction between the solutes and the surfactant head group should decrease and the electrostatic shielding should also reduce the repulsion between surfactant head groups, resulting in smaller micelle (Regalado et al., 1996). Therefore, the salt concentration in the aqueous phase can affect the efficiency of solute transfer through a size exclusion effect (Zhang et al., 1999; Kilikian et al., 2000; Kinugasa et al., 2003). Increasing the salt concentration causes the size of the reverse micelle to decrease and it becomes difficult to incorporate proteins into the water pool of a reverse micelle (Kinugasa et al., 2003). As the salt concentration increases, the attractive interaction diminishes due to Debye screening and the transfer percentage declines (Kinugasa et al., 2003; Yu et al., 2003).

Pires et al. (1996) discussed in detail the effect of increasing salt concentration in reverse micelle extraction. They indicated that increasing the salt concentration of the aqueous phase would reduce the electrostatic interactions as a result of the Debye screening effect which is stronger for larger ions. Thus, at highersalt concentrations, the interactions between hydrophilic biomolecules and the surfactant polar groups are reduced, leading to the formation of smaller micelles. As a consequence of increasing the salt concentration, the solubilisation capacity of the organic phase for water and biomolecules decreases. Pires et al. (1996) also noticed that when the salt 
concentration of the aqueous phase was below a certain limit, reverse micelle and phase separation do not occur. A stable microemulsion is formed. Therefore, the transfer of proteins between phases requires a minimum salt concentration in the aqueous solution. Ichikawa et al. (1992) studied the extraction of proteins using an AOT reverse micellar system. They found that at high salt concentrations, more AOT molecules were required to extract all of the proteins from the initial aqueous phase. Thus, the minimum AOT concentration increased with ionic strength. At higher salt concentrationss, more solubilising water was necessary for extraction of proteins into the reversed micellar organic phase. Therefore, more AOT molecules were required as a water-solubilising agent in order to provide the hydrophilic surroundings for the proteins.

Spirovska and Chaudhuri (1998) and Regalado et al. (1996) studied the effect of salt type on the micelle size and found that the salt type and concentration have a strong influence on $W_{o}$. Nishiki et al. (1993) explored the effects of salt on protein extraction using a reverse micelle system. They found that the extraction of proteins was effected by the concentration of potassium or barium ions but was almost independent of the concentration of sodium or calcium ions, whose ionic diameters are smaller than those of potassium and barium. Similarly, Andrews (1993) in a study of reverse micelle extraction of proteins found that there is an important difference in the value of $W_{o}$ depending on the type of salt present in the aqueous phase. The system that contained $\mathrm{MgCl}_{2}$ resulted in much larger micelle than the system containing $\mathrm{KBr}$. This may be a consequence of the relative sizes of the $\mathrm{K}^{+}$ and $\mathrm{Mg}^{2+}$ ions; smaller ions produce less electrostatic screening of the micelles and hence allow more transfer of solute and water to the micelles. Marcozzi et al. (1991) studied the ionic strength factor using four salts $-\mathrm{KCl}, \mathrm{NaCl}, \mathrm{LiCl}$ and $\mathrm{CaCl}_{2}-$ and found that protein transfer occurred at the lowest ionic strengths with $\mathrm{KCl}$ followed by $\mathrm{CaCl}_{2}, \mathrm{NaCl}$ and $\mathrm{LiCl}$. The atomic radii of these ions decreased in the order $\mathrm{K}^{+}>\mathrm{Ca}^{2+}>\mathrm{Na}^{+}>\mathrm{Li}^{+}$, indicating that the size of the ions may also be important in this system.

Andrews and Haywood (1994) studied the effects of ions of different sizes on the extraction of ribonuclease $\mathrm{A}$ and thaumatin and found that larger ions such as $\mathrm{K}^{+}$ cause more screening, and hence less solubilization, than smaller ions such as $\mathrm{Na}^{+}$. 
Leodidis and Hatton (1990) found that water uptake (expressed in terms of the molar ratio of water to surfactant in the reverse micelle organic phase) with $\mathrm{NaCl}$ was three times greater than with $\mathrm{KCl}$. It is a well known fact that an increase of electrolyte concentration in the aqueous phase causes a decrease in the water solubilised in reverse micelle, and this factor also plays an important role in protein extraction. The extraction of protein also decreases with the addition of salt (Regalado et al., 1996). For example, the addition of $\mathrm{NaCl}$ provides more chloride, which can compete with the protein molecules for reaction with the surfactant head groups. From the above it is clear that salt type greatly affects reverse micelle extraction efficiency.

\subsubsection{Surfactant Concentration}

Surfactant concentration affects protein transfer in the forward extraction to the reverse micelle, and during back extraction. Many studies have revealed that the electrostatic interaction between the oppositely charged surfactant head group present in the reverse micelle and the solute molecule plays a key role in the separation (Pandit and Basu, 2002). A number of studies also show that protein extraction efficiency increases with increasing surfactant concentration. Moreover, Goto et al. (1999) in their study on DNA extraction showed that the degree of extraction was strongly influenced by the type of surfactant in the organic solvent. Zhang et al. (1999) studied the extraction of BSA by using tributyl phosphate (TBP) and trialkyl phosphine oxide (TRPO) surfactants and found, as expected, that the BSA transfer was increased significantly at higher concentrations of both surfactants.

However, there are a numbers of studies which show that high concentrations of surfactants can slow down the extraction of proteins (Pires et al., 1996; Kilikian et al., 2000; Hasmann et al., 2003). Pires et al. (1996) showed that small proteins can be more easily extracted to the micellar phase using low surfactant concentrations than high molecular weight proteins. Ihara et al. (1995) indicated that high concentrations of CTAB are necessary to obtain larger micelle capable of including $\beta$-xyloxidase molecules (molecule size of $100 \mathrm{kDa}$ ), due to the fact that micellar size can be assumed to be approximately proportional to the surfactant concentration. 
They also pointed out that changing the mass of one or more elements can displace the equilibrium, since the reverse micellar phase ceases to exist, which explains the non-recovery of $\beta$-xylaxidase when higher $\mathrm{CTAB}$ and butanol concentrations were used.

When the extraction of antibiotics is not limited by salt concentration and solution $\mathrm{pH}$, the concentration of the extracted proteins initially increases with surfactant concentration until all extractable antibiotics are removed, after which extraction efficiency either drops off or remains at a fixed level. A number of studies have also shown similar behaviours with biomolecules such as proteins (e.g., Nishiki et al., 1993). However, other studies conclude that for the purpose of extraction using reverse micelle, only a low concentration of surfactant is needed. Thus, the extraction of antibiotics at low surfactant concentration is advantageous from a processing standpoint because, according to Goto et al. (1998), amphiphilic molecules such as surfactants are very difficult to remove in subsequent downstream processing steps.

\subsubsection{Biomolecule Size and Concentration}

The size of a protein molecule also influences transfer efficiency in the reverse micelle process. Small proteins are easier to extract than high molecular weight proteins when using low surfactant concentrations (Kilikian et al., 2000). Larger proteins appear to be more difficult to transfer into reverse micelle and therefore the optimum $\mathrm{pH}$ (i.e. the $\mathrm{pH}$ for $50 \%$ transfer) is displaced relative to the isoelectric point. A large cationic protein needs to be transferred at a $\mathrm{pH}$ lower than its isoelectric point while a small protein can be transferred at a $\mathrm{pH}$ very close to its isoelectric point. An empirical equation relating the $\mathrm{pI}$, molecular weight of a protein and a $\mathrm{pH}$ value at which the maximum transfer efficiency occurs has been developed by Wolbert et al. (1989).

Most anions of commercial interest, especially $\beta$-lactam antibiotics, are less easily extracted (Nabais and Cardoso, 1999). High selectivity is difficult to achieve and the 
factors affecting selectivity are not fully understood. There has nonetheless been recent work involving the extraction of antibiotics. Low aqueous phase $\mathrm{pH}$ favours the selective extraction of antibiotics, especially penicillin $G$ to the organic solvent phase, while for $\mathrm{pH}$ values greater than approximately 6.0 the distribution of penicillin is biased towards the aqueous phase; this result is a consequence of penicillin being a strong organic acid (Hatton, 1985). Erythromycin, on the other hand, is an organic base, and is extractable with organic solvents only under basic, rather than acidic conditions. Other antibiotics, such as streptomycin, are highly polar solutes and under normal circumstances do not show appreciable extraction with organic solvents. The antibiotics previously studied have different structures and therefore have different equilibrium constants in ion exchange reactions (Rabie and Vera, 1996). It can be postulated that an antibiotic with a lower molecular weight has a greater tendency to react with the surfactant and thus a higher ion exchange reaction equilibrium constant.

The effect of biomolecule initial concentration on thermodynamic partitioning in a reverse micelle system is an important factor, but few studies have been carried out to date. Leser and Luisi (1990) studied the effect of increasing initial protein concentration and found that there exists a saturation point at which an increase in initial protein concentration does not affect the maximum transfer efficiency obtained. The same results are expected for the partitioning of antibiotics in a reverse micelle system.

\subsubsection{Solvent Type}

The properties of an organic solvent can significantly influence the reverse micelle extraction of an antibiotic. Hydrophobicity is one of the important properties and quantified by taking the logarithm of the distribution coefficient $(\log K) . \log K$ is defined as the logarithm of the distribution coefficient between the organic solvent and water, i.e.: 


$$
\log K=\log \frac{[\text { solvent }]_{\text {organic }}}{[\text { water }]}
$$

Table 2.2 shows a range of solvents which can be used to form reverse micelle; most of them are aliphatics. The $\log K$ value is determined experimentally from the standard octanol-water system or calculated by summation of the hydrophobic fragmental constants of its constituents. Mat and Stuckey (1994) concluded that with a more non-polar solvent, the more likely it is that a surfactant will aggregate and form reverse micelle.

A more relevant study was done by Feng (1995) to determine the effect of the solvent type in the extraction of soybean peroxidase (SBP) using reverse micelle. No extraction was observed in hydrophilic solvents (log $K \leq 2)$ or in hydrophobic solvents such as octanol and nonanol, whereas alkanes with $\log K$ values $>3.5$ such as hexane supported higher degrees of extraction (Table 2.3). SBP was inactive in the solution contacted with hydrophilic solvents. The lack of SBP facilitated extraction in octanol and nonanol, which have $\log K$ values comparable to that of hexane, may be explained by the tendency of alkanols to act as co-surfactants.

Table 2.2: Range of solvents used (Mat and Stuckey, 1994). Solubility refers to solubility of the solvents in an aqueous phase in $\%(w / w)$.

\begin{tabular}{|c|c|c|}
\hline Solvent & Log $\mathrm{K}$ & \% Solubility $(\mathrm{w} / \mathrm{w})$ \\
\hline 2,2 dimethylbutane & 3.3 & 0.0018 \\
Cyclohexane & 3.2 & 0.0055 \\
Hexane & 3.5 & 0.0012 \\
Heptane & 4.0 & 0.0004 \\
Octane & 4.5 & $6.6 \times 10^{7}$ \\
Isooctane & 4.5 & 0.0002 \\
Decane & 5.6 & $52 \mathrm{ppb}$ \\
Dodecane & 6.6 & $3.7 \mathrm{ppb}$ \\
Tetradecane & 7.6 & - \\
Hexadecane & 8.8 & - \\
p-xylene & 3.1 & 0.0156 \\
Benzene & 2.0 & 0.1791 \\
Carbon tetrachloride & 3.0 & 0.0770 \\
Toluene & 2.5 & 0.0515 \\
Chloroform & 2.1 & 0.8150 \\
\hline
\end{tabular}


Table 2.3: Extraction of SBP employing different organic solvents as feed phases (Feng,

1995).

\begin{tabular}{|c|c|c|}
\hline Solvent & Hydrophobicity, $(\log K)$ & \% Extraction of SBP \\
\hline Ethyl acetate & 0.68 & 0 \\
Amyl alcohol & 1.3 & 0 \\
Toluene & 2.5 & 0 \\
Hexane & 3.5 & 42.2 \\
Octane & 4.5 & 75.8 \\
Nonane & 5.1 & 88.1 \\
\hline
\end{tabular}

\subsubsection{Other Factors that Influence Partitioning}

Two other factors affecting solubilisation of biomolecules in reverse micelle are temperature and phase volume ratio $\left(V_{r}=V_{a q} / V_{r m+o}\right)$ at which the extraction is carried out, where $V_{a q}$ the volume of aqueous phase and $V_{r m+o}$ the volume of reverse micelle organic phase. Shin et al. (2002) showed that a change in temperature from room temperature to $35^{\circ} \mathrm{C}$ has a negligible effect on the solubilization limit of lysozyme into a DODMAC reverse micellar phase. For the volume ratio effect, $\mathrm{Yu}$ et al. (2003) observed that the extraction yield increased at higher phase volume ratio when the aqueous phase volume was fixed. They found that increasing the reverse micellar phase volume would increase the number of reverse micelle and thereby the mass of lipase extracted would increase. However, Shin et al. (2003) argued that an increase in the phase volume ratio could increase the amount of counterions in contact with the organic phase, and reduce the extraction efficiency. They noted that this phenomenon indicates that solubilization of biomolecules in the reverse micellar phase is not controlled by phase equilibrium, but by chemical equilibrium.

\subsubsection{Backward Extraction Process}

Antibiotic extracted into the reverse micelle must subsequently be transferred from the reversed micellar organic phase into an aqueous stripping solution for recovery as the purified and enriched product. This is referred to as the backward extraction process. Although no work could be found on the reverse micelle backward extraction of antibiotics, there have been several reports regarding the back 
extraction of proteins (Woll and Hatton, 1989; Carlson and Nagarajan, 1992; Nishiki et al., 1995; Ono et al., 1996; Nishiki et al., 1998; Zhang et al., 1999; Hong et al., 2000; Kilikian et al., 2000; Xun et al., 2001; Yu et al., 2003; Bong et al., 2004; Jun et al., 2004; Mathew and Juang, 2007).

It has been established that the rate of backward transfer of protein from a reverse micelle to an aqueous phase is relatively slow. The backward process was reported to be three orders of magnitude slower than for forward extraction (Dungan et al., 1991; Zhang et al., 2002) and more difficult to accomplish (Kinugasa et al., 1991; Ono et al., 1996; Pires et al., 1996) due to the large interfacial resistance in mass transfer (Dungan et al., 1991; Kinugasa et al., 1991; Ono et al., 1996). Hong et al. (2000) asserted that the problems with backward extraction originate from the structural change of proteins and micelle due to their strong interactions. The strategy for improvement could consider three aspects. One deals with the choice of surfactant for compatibility with the organic phase by concentration and species of surfactant or type of organic solvent. Another with the composition of the backward aqueous phase by $\mathrm{pH}$, concentration and species of salts or adding various alcohols, whilst the third deals with temperature or pressure of the whole system.

The backward extraction is known to depend on the $\mathrm{pH}$ value of the feed solution which is a fresh aqueous phase contacted with the reverse micellar organic phase (Ono et al., 1996; Bong et al., 2004). In backward extraction, the pH value must be such that the protein has the same charge as the surfactant molecules: in this way repulsion forces are created which cause the release of protein from the reverse micelle (Kilikian et al., 2000; Yu et al., 2003). The protein extracted into the reverse micelle can also be recovered by backward transfer through the size exclusion effect which is brought about by increasing the salt concentration in the fresh aqueous phase (Ono et al., 1996). Jun et al. (2004) found that in order to decrease the electrostatic interactions between nattokinase and AOT, the $\mathrm{pH}$ and ionic strength of the backward aqueous phase needed to be increased. Ionic species and ionic strength of the fresh backward aqueous phase are also important factors for backward transfer. For the nattokinase/AOT system increasing the ionic strength led to a decrease in electrostatic interaction and promotion of backward transfer. Kilikian $e t$ al. (2000) noted that high ionic strength is desirable in the new aqueous phase where 
the backward extraction is performed, and the backward extraction yield decreases with factors that decrease the droplet radius of the reverse micelle, such as an increase in salt concentration.

Ono et al. (1996) found that the addition of 10-15\% isopropyl alcohol could improve the solubilisation of protein in the backward extraction process without destroying the functional integrity of a reverse micelle. Carlson and Nagarajan (1992) obtained an almost complete backward extraction of porcine pepsin and also a $70 \%$ back transfer of bovine cymosin when an amount of alcohol was added during the process. Each alcohol has a different critical alcohol concentration (CAC) for the release of haemoglobin from the reverse micellar phase into the recovery phase, and further, the $\mathrm{CAC}$ decreases gradually with increasing carbon number in the alcohol (Ono et al., 1996). However, Pires et al. (1996) asserted that only a moderate addition of alcohol promotes haemoglobin release, and that reverse micelle might be destroyed by a larger amount of alcohol. Pires et al. (1996) also noted that alcohol type also an important factor. They found that back extraction of lipase $B$ from the reverse micellar phase was only achieved by introducing a water-miscible organic solvent. They obtained the best results by adding $2.5 \%$ ( $/ / \mathrm{v}$ of the total volume of the phases) ethanol to the micellar phase at $\mathrm{pH} 9.0$ using the same ionic strength as for extraction. Ethanol was used to minimise the hydrophobic interactions between the lipase and the surfactant and/or organic solvent, allowing its recovery into the aqueous phase.

\subsubsection{Thermodynamic Framework of Partitioning}

Understanding phase equilibria in reverse micelle systems is important for designing downstream processing; to aid this, thermodynamic modelling for interpretation of phase equilibria is necessary. Several thermodynamic models have been implemented for protein partitioning from the aqueous phase into the reverse micellar phase. Bratco et al. (1988) were the first workers to develop a thermodynamic model for partitioning of protein which noted that solubilisation is a function of $\mathrm{pH}$ and ionic strength. Bruno et al. (1990) agreed that $\mathrm{pH}$ and ionic 
strength are the functional parameters in the partitioning of protein. Woll and Hatton (1989) presented a simple phenomenological model for protein partitioning in an anionic reverse micelle system, and showed the dependencies of the partition coefficient of proteins between the two phases. They assumed a pseudochemical equilibrium for solubilisation of the protein molecules with the hydrophobic tails of reverse micelle as empty sites. They found that $\mathrm{pH}$ is the most important parameter to affect protein partitioning. Rabie and Vera (1998) developed an ion exchange model for protein partitioning, which focussed on the reaction between protein molecules and the surfactant counter ion. They found that at equilibrium, the protein concentration in both phases is the same and the activity of components in the system is equal to its concentration.

The most recent model developed by Haghtalab and Osfouri (2003) uses 'vacancy solution theory' based on a surface pressure. On the premise that one vacancy solution represents the aqueous phase and the other the reverse micellar phase, Haghtalab and Osfouri (2003) incorporated a surface pressure to model protein adsorption in reverse micelle and the non-ideality of the system was expressed in terms of the Wilson activity coefficient model. The concentration of protein remaining in the aqueous phase was obtained as:

$$
\left[P_{r}\right]_{a q . f}=\left[\frac{1}{b} \frac{\theta_{1}}{1-\theta_{1}}\right] f\left(\Lambda_{12}, \Lambda_{21}, \theta_{1}\right)
$$

where

$$
f\left(\Lambda_{12}, \Lambda_{21}, \theta_{1}\right)=\Lambda_{12} \frac{1-\left(1-\Lambda_{21} \theta_{1}\right)}{\Lambda_{12}+\left(1-\Lambda_{12}\right) \theta_{1}} \exp \left[\frac{-\Lambda_{21}\left(1-\Lambda_{21}\right) \theta_{1}}{1-\left(1-\Lambda_{21}\right) \theta_{1}}-\frac{\left(1-\Lambda_{12}\right) \theta_{1}}{\Lambda_{12}+\left(1-\Lambda_{12}\right) \theta_{1}}\right]
$$

where $\Lambda_{12}$ and $\Lambda_{21}$ are Wilson parameters and $\theta_{1}$ is the fractional coverage of the protein molecules. The term in square brackets is exactly the same as the Langmuir equation, and $f\left(\Lambda_{12}, \Lambda_{21}, \theta_{1}\right)$ may be regarded as a correction factor to the Langmuir isotherm. The data of Haghtalab and Osfouri (2003) showed that the values of the Wilson parameters are $\mathrm{pH}$ dependent, which could reasonably be expected since the 
surface charge on the protein (BSA in their case) varied from negative at $\mathrm{pH}$ values above the $\mathrm{pI}$ to positive at values below the pI.

Brandani et al. (1994) presented the concept of an 'adsorption model' which is based on the assumption that reverse micelle are the active sites for the adsorbing biomolecule (protein - in their case) from aqueous solution. The model used a Langmuir adsorption isotherm to describe the partitioning of protein molecules between the two phases. The theory was able to identify the interfacially active biomolecule (amino acid - in their case).

In the current work, the adsorption theory of Brandani and co-workers has been used. According to Brandani et al. (1994), concentration of solute in the reverse micelle organic phase can be expressed as,

$$
[P]_{r m+o . f . f w}=\frac{a_{B} b_{B}[S][P]_{a q . f . f w}}{1+b_{B}[P]_{a q . f . f w}}
$$

where $a_{B}$ and $b_{B}$ are both adjustable parameters; $b_{B}$ is a parameter characteristic of the inner surface of reverse micelle and independent of $[P]_{a q . f f w}[S]$ is the concentration of surfactant.

A form of the Langmuir equation for concentration of solute in the reverse micelle can be expressed as:

$$
[P]_{m+o . f . f w}=\frac{a_{L}[P]_{a q . f . f w}}{1+b_{L}[P]_{a q . f . f w}}
$$

A useful theory of 'qualitative measures of interfacial association' by Leodidis and Hatton (1990) was obtained by observations of the manifestations of the interfacial phenomena between reverse micelle and aqueous phase. They use a $45^{\circ}$ line in a plot of $[P]_{\text {aq.ffiw }}$ versus $[P]_{\text {aq.ifw }}$ and showed that if the data fell on the line, the biomolecules used are non interfacially active. The authors also used another method to identify the interfacially active biomolecule by plotting $t_{a}$ (the biomolecule transfer) versus $t_{w}$ (the water transfer). 


$$
\begin{aligned}
& t_{w}=1-\frac{V_{a q . f}}{V_{a q . i}} \\
& t_{a}=1-\frac{[P]_{a q . f} V_{a q . f}}{[P]_{a q . i} V_{a q . i}}
\end{aligned}
$$

These expressions are attractive because of their relative simplicity and arise from mass balances on experiments carried out in the laboratory.

\subsection{Conclusions}

This chapter has presented a literature review of separation methods used in the pharmaceutical industry and reverse micelle systems. It is observed that several methods can be used in the extraction of pharmaceutical products such as a conventional solvent extraction, an aqueous two phase process, liquid membranes, chromatography, and reverse micelle extraction. The commercial production of bioproducts currently uses solvent extraction and the method is well established in the downstream processing of bioproducts e.g. antibiotics. Although most of the extraction methods discussed have potential to be utilised for the downstream processing of bioproducts, many have significant drawbacks which require further research before large scale application can be considered.

One of the extraction methods that has shown potential is a system that utilises micelle. The reverse micelle system has attracted much attention as a potential technique for separating and purifying biological products. The system has been proven to be successful in the extraction of proteins and enzymes without causing denaturation, although adjustment of operational parameters is generally required. However, more studies need to be conducted to aid better understanding. 


\section{CHAPTER THREE \\ Experimental Materials and Methods}

\subsection{Introduction}

This chapter addresses the materials, characterization techniques and experimental procedures used to obtain results for the measurement of feed solution characteristics, forward extraction and backward extraction.

\subsection{Materials}

Reagent grade sodium di-2-ethylhexyl sulfosuccinate (AOT) was used as the anionic surfactant; and has the chemical structure is shown in Figure 3.1. The organic solvent used for the reverse micelle phase was analytical grade isooctane $(2,2,4-$ trymethylpentane). The biomolecule was analytical grade penicillin $\mathrm{G}$ sodium salt; the structure of the salt is shown in Figure 3.2. $\mathrm{CaCl}_{2}$ and $\mathrm{KCl}$ were used as salts in the aqueous phase. All chemicals were supplied by Sigma Aldrich UK and were used as received. All experiments were conducted at a temperature of $23 \pm 1^{\circ} \mathrm{C}$.

The aqueous phases were made up using double distilled water and reagent grade potassium chloride $(\mathrm{KCl}) ; \mathrm{HCl}$ and $\mathrm{NaOH}$ were used to adjust solution $\mathrm{pH}$. Use of buffer to adjust $\mathrm{pH}$ was avoided since the buffer interferes with the extraction of penicillin G. Both the buffer and penicillin $G$ are weak salts and their degree of dissociation is a function of $\mathrm{pH}$. The buffer anions may also compete with the penicillin $G$ in the extraction process. De-ionised water was used in the cleaning of all equipment to remove any microorganisms or residues of biological materials that might still be attached to the equipment. 


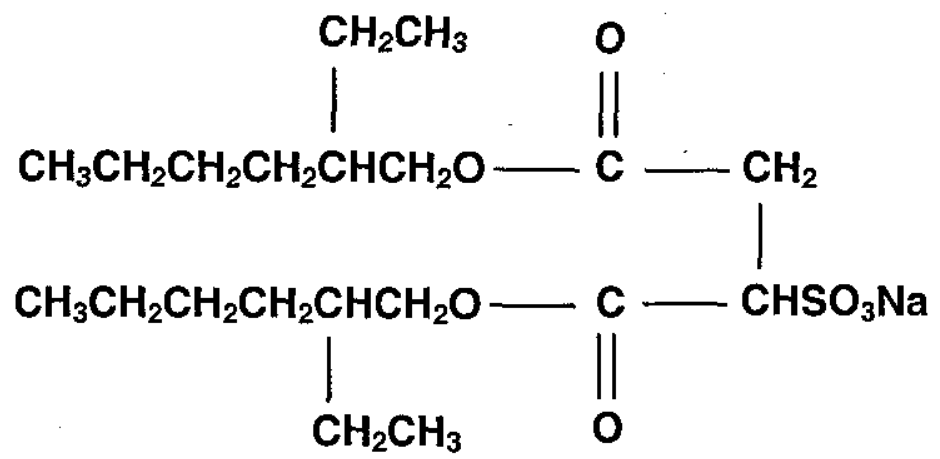

Figure 3.1: Structure of sodium di-2-ethylhexylsulphosuccinate (AOT).



Figure 3.2: Structure of penicillin G.

\subsubsection{Penicillin G}

Penicillin $\mathrm{G}$ is a widely used antibiotic which also serves as a raw material for semisynthetic penicillin. Penicillin G and Penicillin F were the first of the penicillin group to be discovered from the culture filtrate of Penicillium chrysogenum. These naturally occurring penicillins contain the 6-aminopenicillanic acid (6-APA) nucleus. The natural penicillins vary considerably in their intrinsic antibacterial activity. The high activity of penicillin $\mathrm{G}$ against Pyogenic cocci has made it a drug of choice. In general penicillin $G$ is the most effective of the other natural penicillins against spirochaetal infections.

Penicillin $G$ is a weak acid that is lipophilic and extremely unstable. Physical extraction at $\mathrm{pH} 2.0$ to 2.5 is currently employed to recover penicillin $\mathrm{G}$ from the 
broth, but about $10 \%$ losses of penicillin occur because penicillin $\mathrm{G}$ is unstable and tends to decompose in the low $\mathrm{pH}$ range and ambient temperature.

\subsection{Feed Solution Characteristics}

Several analysis methods were used to characterise the feed solution:

- The critical micelle concentration (CMC) of AOT solution was determined with and without penicillin $\mathrm{G}$ using the Wilhelmy Ring method.

- The Kjeldahl method was used to determine the mass of penicillin $G$ in the aqueous solution after extraction. The mass of penicillin $G$ in the reverse micelle (organic phase) was calculated from a mass balance.

- The water content of the organic phase, both with and without penicillin $G$, was determined using the Karl Fisher titration method.

- A calibrated $\mathrm{pH}$ meter was used to determine $\mathrm{pH}$ of the aqueous solutions before and after the extraction process.

\subsubsection{Mass and Concentration of Penicillin G}

The mass and concentration of penicillin $G$ in the aqueous phase after the extraction process was determined using the Kjeldahl Method. For the solutions used in this work, only penicillin $\mathrm{G}$ contained nitrogen within its molecule structure, so the mass of nitrogen in the solution represents the mass of penicillin G. From the mass of nitrogen the concentration of penicillin $\mathrm{G}$ in the solution could also be calculated. A Buchi nitrogen determination system was used to measure nitrogen content in a sample of penicillin G (Figure 3.3). The system contains a Buchi 323 distillation unit, a Buchi 412 scrubber unit, and a B-426/B-435/B-436 digestion unit. The three steps involved in this method are digestion, distillation and titration. 


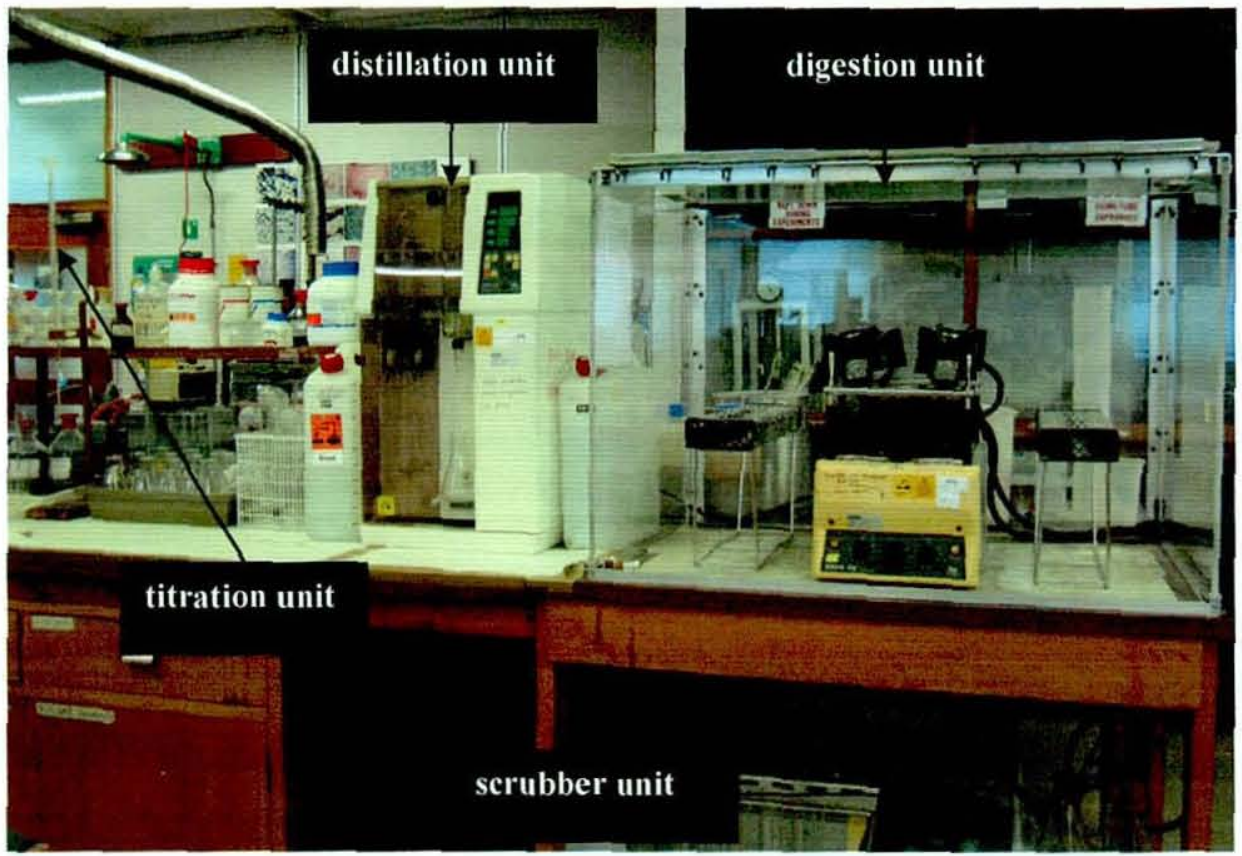

Figure 3.3: Nitrogen analyzer.

The reagents required for the digestion step are concentrated sulphuric acid (analytical grade) and Kjeldahl catalyst tablets $\left(1 \mathrm{~g} \mathrm{~K}_{2} \mathrm{SO}_{4}+0.1 \mathrm{~g} \mathrm{CuSO}\right.$ ). For the distillation step, the reagents required are $\mathrm{NaOH}(32 \%$ in a 10 litre container), distilled water for steam generation and digester dilution (in a 10 litre container), 50 $\mathrm{mI}$ boric acid solution ( $2 \%$ ) plus an indicator (bromocresol green and methyl red) and $\mathrm{HCl}$ (volumetric $0.1 \mathrm{M}$ ).

To begin the analysis (digestion step), a $5 \mathrm{ml}$ sample of the aqueous phase was placed into a Kjeldahl tube and $10 \mathrm{ml}$ of sulphuric acid was poured into the tube using a tilting dispenser. Two tablets of the Kjeldahl catalyst were added to the solution to facilitate digestion in the digester units. Six or 12 tubes may be used, but all places in any rack must be filled. This is important as the extracter/scrubber unit can only operate efficiently when all tubes are fitted on the manifold(s). Immediately after addition of the catalyst tablets, the tube was heated with the tube mouth over the blower. After all the water was boiled off, the sample begins to smoke. Digestion time depends on the nature of the sample, but was typically complete in 30 to 40 minutes. When this process was completed, the digestion samples appeared green and clear. The tubes were then allowed to cool for 30 minutes. 
The second step was distillation. $50 \mathrm{ml}$ of boric acid (2\%)-mixed indicator solution was prepared in a conical flask and placed in the distillation unit. The digest tube was placed in the unit and pushed against the seal to allow the clamping device to be locked and the safety door closed. The distillation unit automatically diluted the content of the tube with $110 \mathrm{ml}$ sodium hydroxide (32\%) and $100 \mathrm{ml}$ distilled water after the start button was pressed. When the distillation process was completed, the indicator in the boric acid changed colour from grey to green. The last step was titration, in which $0.05 \mathrm{M} \mathrm{HCl}$ was added to the titration pipette. The solution was titrated until the original purple colour of the boric acid indicator reappeared.

\subsubsection{Surface Tension}

A Wilhelmy Ring method was used to determine the critical micelle concentration (CMC) of the AOT solution (in isooctane), both with and without penicillin G. The surface-active molecules of AOT in solution are capable of displacing water molecules at the surface. The displacement of water molecules and their subsequent replacement by the surface-active molecules leads to a reduction in the surface tension. When the surfactant continues to displace water molecules at the water-air interface there is a continuous decrease in the surface tension of the solution until the surfactant molecules create monolayer coverage at the surface. Further increase in the concentration of surfactant causes a gradual change in the surface tension. The surfactant molecules begin to aggregate to form reverse micelle after the surface is saturated. Figure 3.4 shows a plot of surface tension versus concentration of surfactant. The plot shows a decrease slope followed by a more gradual slope after the interface is saturated with surfactant. The critical micelle concentration of the surfactant is the point at which further increase in the concentration of the surfactant causes no substantial change in the surface tension. 


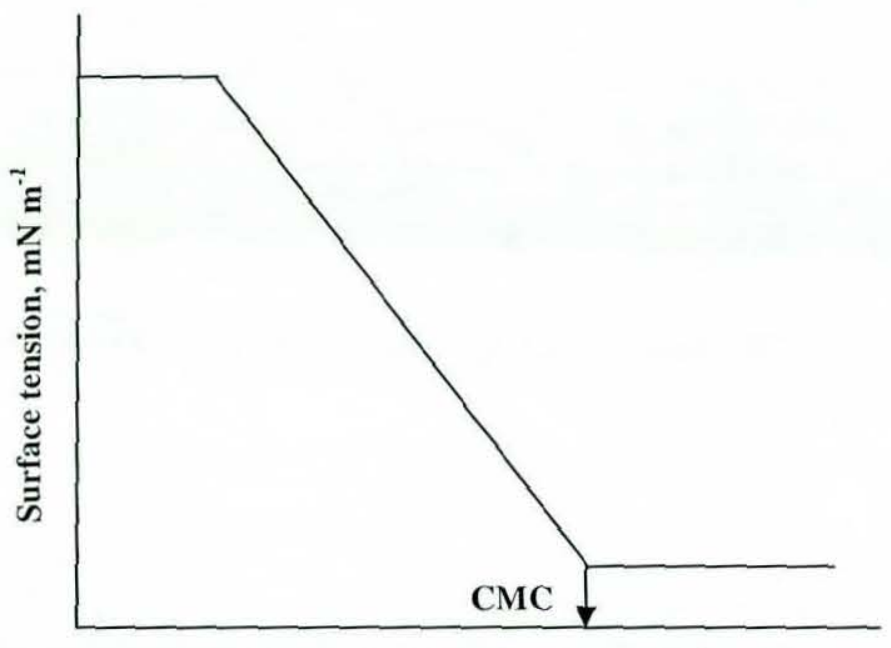

Concentration of surfactant, $[S](\mathrm{g} / \mathrm{l})$

Figure 3.4: Schematic plot of surface tension vs. concentration of surfactant.

A stock solution of $250 \mathrm{~g} / \mathrm{l}$ of AOT in isooctane was prepared. Different concentrations of AOT solutions ranging from 5 to $250 \mathrm{~g} / \mathrm{l}$ were made by diluting this stock with appropriate volumes of pure isooctane. A $3.6 \mathrm{~g} / \mathrm{l}$ stock solution of penicillin $\mathrm{G}$ in $\mathrm{KCl}$ was also prepared. $5 \mathrm{ml}$ samples of each solution were mixed together for 10 minutes and allowed to separate and form clear phases. Surface tension measurements in the systems were performed by the ring method for the organic phase, using a Wilhelmy Ring connected to a DB2ks tensiometer supplied by White Electrical Instrument Co. Ltd, England (Figure 3.5).

In a determination of surface tension, a small quantity of the AOT solution was placed in the concave dish on the pan support arm and the pan was raised so that the ring was completely immersed in the liquid but close to the surface. The surface tension value for the sample being measured was the value recorded on the tensiometer at the point at which the platinum ring broke away from the liquid, as the concave dish was continuously lowered. To achieve good results, the platinum ring was cleaned before each test using a solution of chromic acid. Each sample was measured five times and the average of the five values was taken as the surface tension value for that particular sample. The cleaning process involved washing the ring with de-ionised water, followed by dipping it in a solution of chromic acid and 
then washing the acid from the ring with de-ionised water. The ring was subsequently dipped into ethanol and dried in a fine Bunsen burner flame. After this cleaning step the ring was ready for use again.

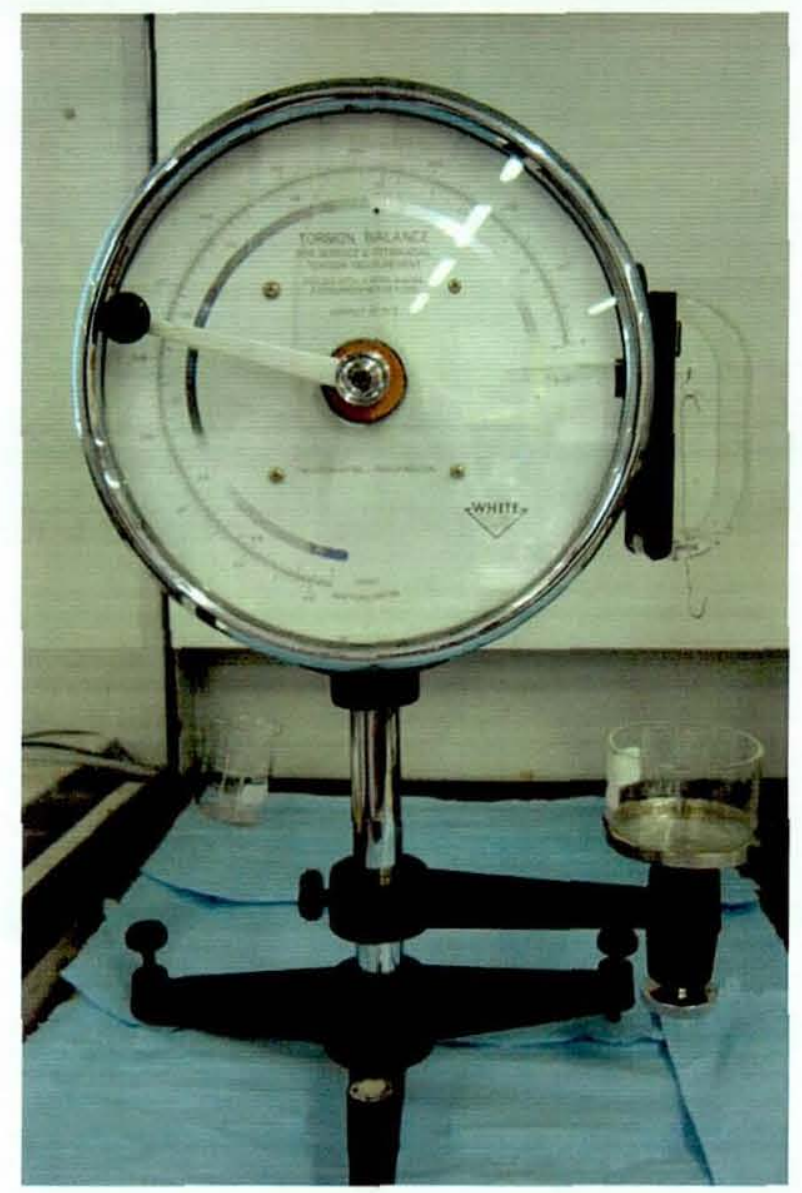

Figure 3.5: Surface tension unit.

\subsubsection{Water Content}

The water content in the reverse micelle (organic phase) was measured using a Karl Fisher (KF) titrator, CA-20 Moisturemeter, Mitsubishi Kasei Corporation (Figure 3.6). The anode solution used was Aquamicron-AS Solution A and the cathode solution was Aquamicron-CS. The readings from the KF titrator given as percent by weight of water in the sample were converted to the water uptake, $W_{o}$, which indicates the molar ratio of water to surfactant in the organic phase, $\left[\mathrm{H}_{2} \mathrm{O}\right] /[S]$. 
During a measurement, electrolyte containing iodide ions, sulphur dioxide, a base and an alcohol is electrolysed in a titration cell to generate free iodine. The iodine reacts with water to produce hydrogen iodide according to the following equation:

Water iodine reaction

$$
\mathrm{H}_{2} \mathrm{O}+\mathrm{I}_{2}+\mathrm{SO}_{2} \rightarrow \mathrm{HI}+\mathrm{SO}_{3}
$$

Generation of iodine

$$
2 I^{-}-2 e \rightarrow I_{2}
$$

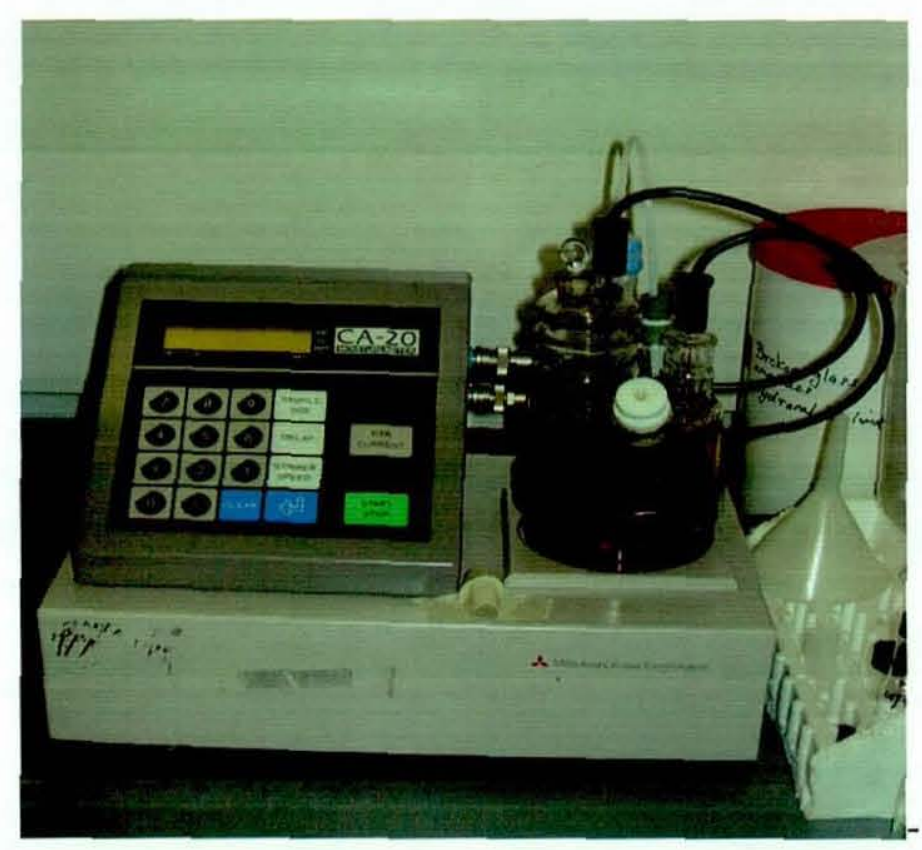

Figure 3.6: Karl Fischer titration unit.

By using an appropriate syringe and good analytical technique, a small quantity of reverse micelle solution was drawn into a syringe. A silicon rubber cover was put on the needle tip and the syringe weighed using a five decimal place balance. The weight was recorded and the silicon rubber removed before the sample was injected into the titration cell through a rubber packing. The syringe was then removed and the silicon rubber cover was replaced on the needle tip before the syringe was reweighed to get the final mass value. 


\subsection{4 pH}

The $\mathrm{pH}$ of the aqueous solution was measured using a Mettler-Toledo 340 digital $\mathrm{pH}$ meter. The final $\mathrm{pH}$ in the aqueous phase was also measured to ensure that no significant $\mathrm{pH}$ change occurred after contacting the two phases. The maximum $\mathrm{pH}$ change between initial and final values was always less than 0.5 units, which is considered acceptable for this work.

\subsection{Forward Extraction Experimental Procedure}

The method used in this research is developed from studies relating to the reverse micelle extraction of proteins done by other researchers (e.g., Ono et al., 1996; Goto et al., 1998; Shen and Yu, 2007; Debnath et al., 2007). The forward extraction experiments involved the extraction of penicillin $G$ from an aqueous phase containing $\mathrm{KCl}$ and penicillin $\mathrm{G}$ to an organic phase containing AOT and isooctane. The phase transfer experiments are shown diagrammatically in Figure 3.7.

$5 \mathrm{ml}$ of aqueous solution containing penicillin $\mathrm{G}$ and $\mathrm{KCl}$ were contacted with an equal volume of isooctane containing AOT; in a sequence of experiments different AOT concentrations, $\mathrm{pH}$, and penicillin $\mathrm{G}$ concentrations were used. The $\mathrm{pH}$ of the aqueous phase was adjusted using either $\mathrm{NaOH}$ or $\mathrm{KOH}$. The solutions were then mixed for 10 minutes using a magnetic stirrer at $400 \mathrm{rpm}$. Higher stirrer speeds and longer stirring periods were not used since a stable emulsion formed under these conditions. The mixture was then left for the phases to separate. After 24 hours of settling (a minority of samples needed more than 24 hours to produce a clear interface between the aqueous and organic phases) samples of the organic phase were removed carefully using a pipette or syringe. For each experiment a blank solution was also prepared by contacting an aqueous solution without penicillin $G$ with an equal volume of initial organic solution containing reverse micelle. After settling, all samples of both phases were transparent to the naked eye. All experiments were replicated two to four times in order to assess reproducibility of the results. 
The final aqueous solution was analysed using the Kjeldahl method. Since nitrogen existed only in the penicillin $\mathrm{G}$, the nitrogen content could be calibrated against the penicillin $\mathrm{G}$ concentration. A $10 \mu \mathrm{l}$ sample of the reverse micelle solution was used to measure the water content using the Karl Fischer titration method.

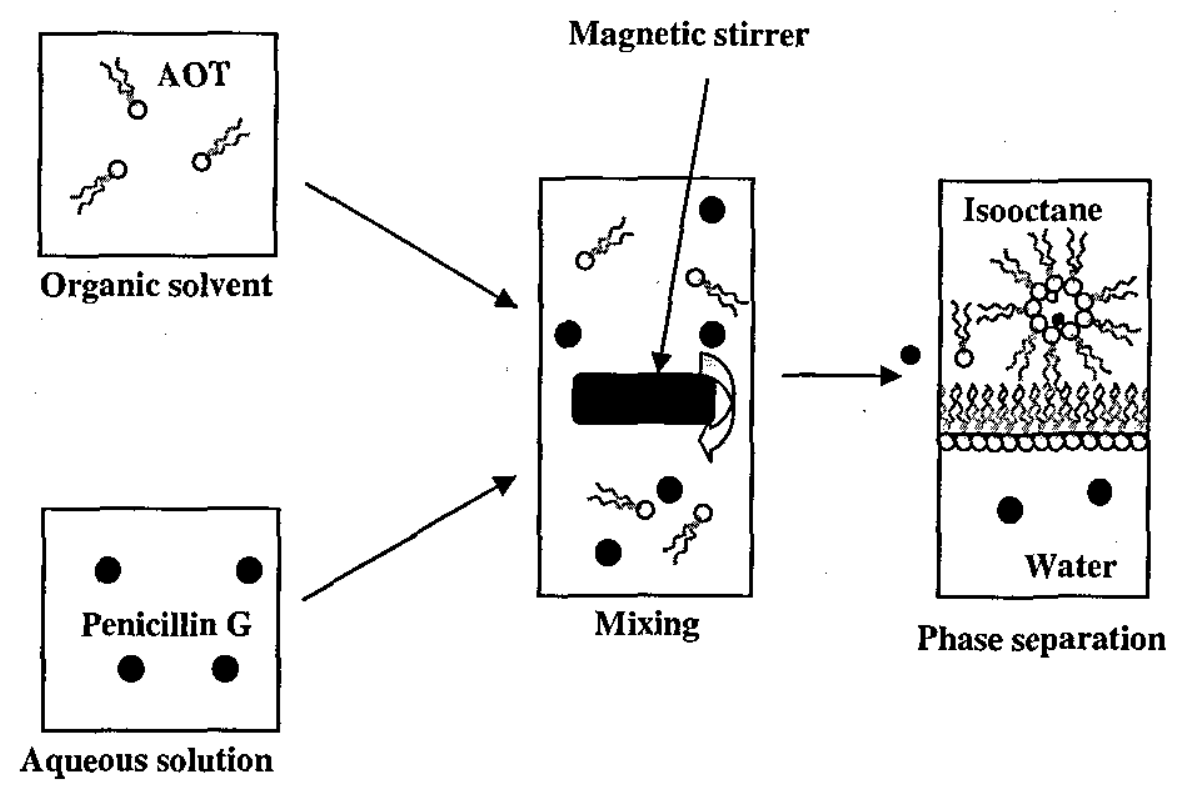

Figure 3.7: Forward extraction by reverse micelle.

The most suitable mixing time for the forward transfer of penicillin $G$ was determined through a series of experiments. It was important for the correct mixing time to be used for the transfer to enable the system to achieve equilibrium. An aqueous phase consisting of $0.1 \mathrm{M} \mathrm{KCl}$ and $10 \mathrm{mM}$ penicillin $\mathrm{G}$ was prepared at $\mathrm{pH}$ 7 and room temperature. An organic phase with of $10 \mathrm{mM}$ surfactant was also prepared. $25 \mathrm{ml}$ volumes of each were mixed together for 20 minutes. While mixing was taking place, $5 \mathrm{~mL}$ of the resultant mixture were taken out at different time intervals with syringe and allowed to reach equilibrium at room temperature. The aqueous phase was collected for further analysis using the nitrogen analyser to determine the penicillin $\mathrm{G}$ content after the extraction process. 


\subsection{Backward Extraction Experimental Procedure}

Backward extraction was performed with an aqueous solution of desired $\mathrm{pH}$ and ionic strength as a stripping solution. After the aqueous phase from a forward extraction experiment was removed completely with a syringe, the stripping solution was added and contacted with the organic phase obtained from the forward extraction using the same procedure as that describe for forward extraction (Section 3.4), except that a shorter period of mixing was used (5 minutes) (Figure 3.8).

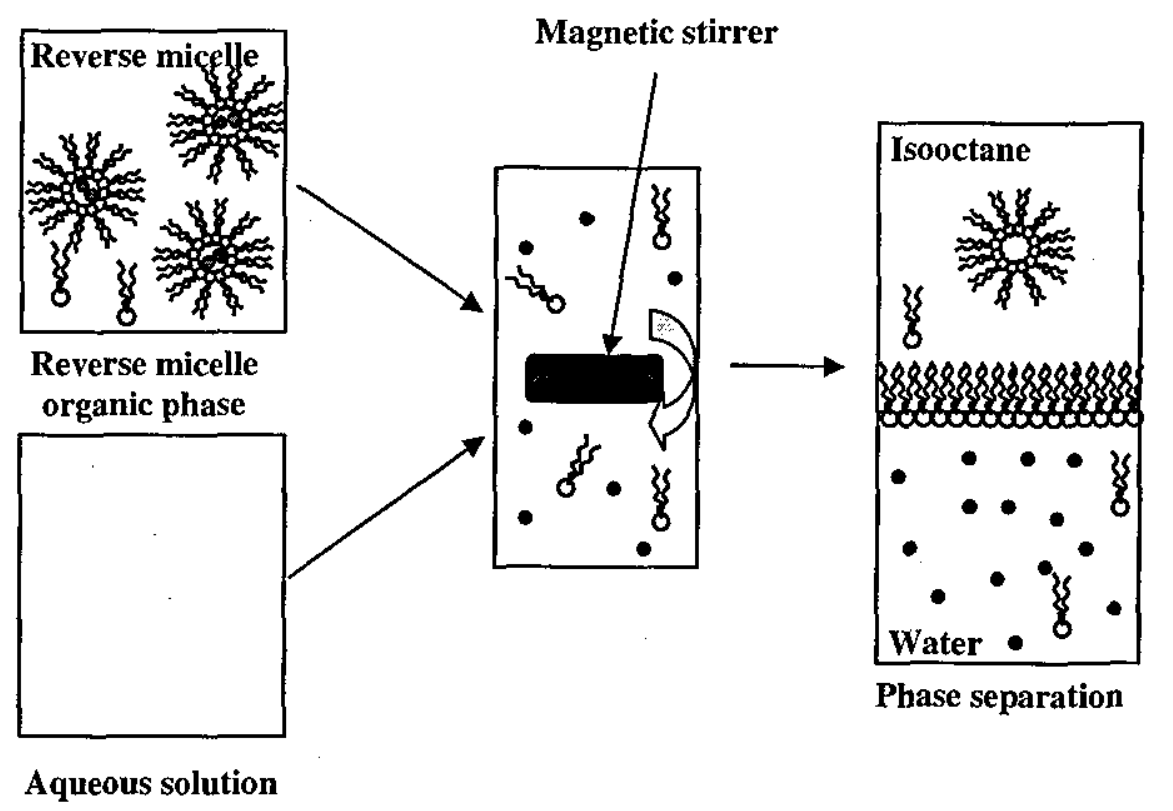

Figure 3.8: Backward extraction from reverse micelle.

The mixture was then left for the phases to separate. After settling, samples of the organic phase were removed carefully using a pipette or syringe. All samples of both phases were transparent to the naked eye. The aqueous solution was measured using the nitrogen analyser. In similar manner to the forward extraction process, higher stirring speed and longer stirring period were avoided. An organic solution that had not been contacted with a new aqueous phase was used as the blank solution for the backward extraction. After settling, all samples of both phases were visually checked for clarity. All experiments were replicated two to four times in order to assess the reproducibility of the results. The flow chart of the experimental procedures of both forward and backward extraction processes is shown in Figure 3.9 . 


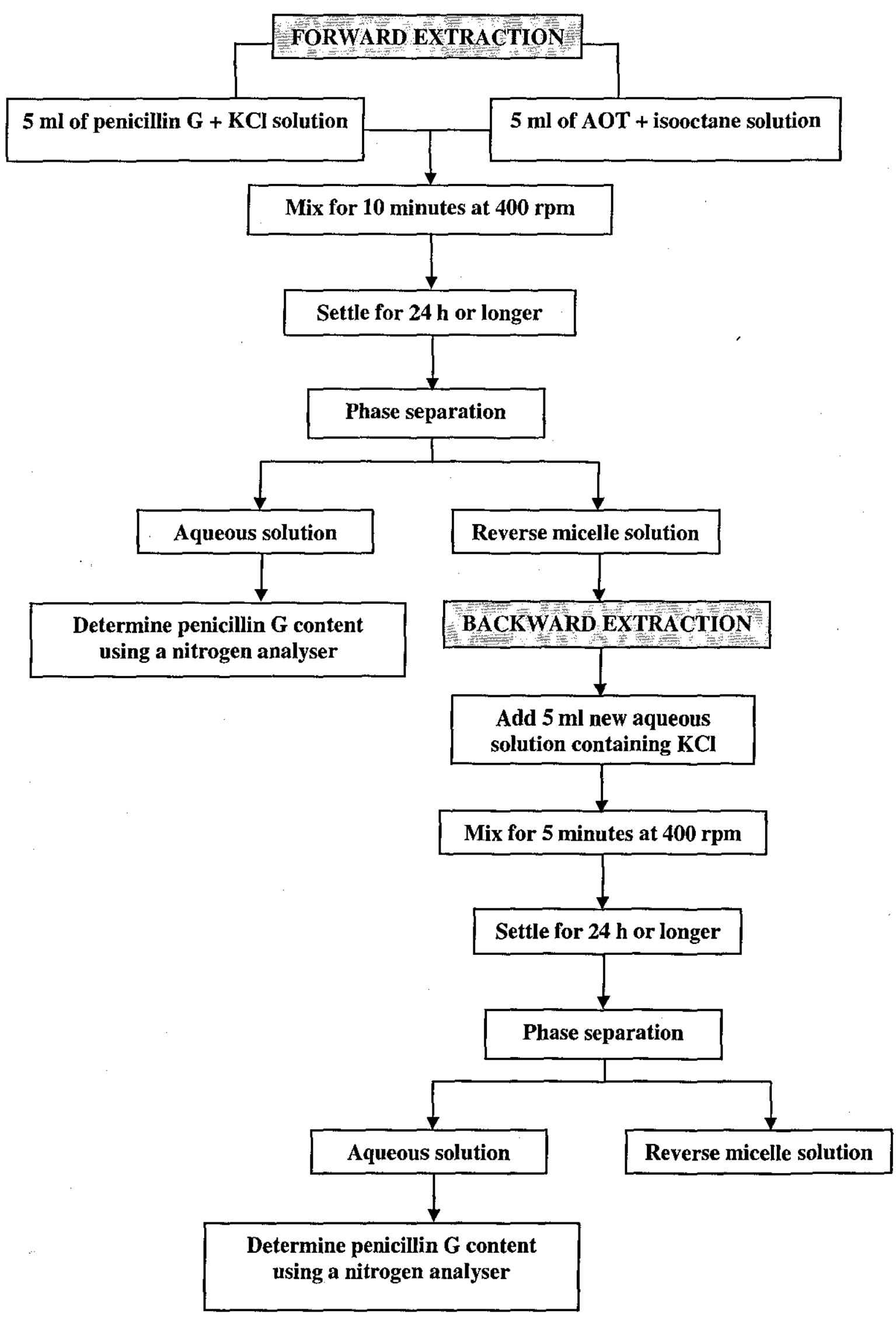

Figure 3.9: Flow chart of the experimental procedures for forward and backward extraction. 


\subsection{Penicillin G Content Determination}

\subsubsection{Calibration Curve}

The concentration of penicillin $\mathrm{G}$ in the aqueous phase after the extraction process was measured using the Kjeldahl method. From the structure of penicillin $\mathrm{G}$ (see Figure 3.2), the percentage of nitrogen in a molecule is about $7.7 \%$. A calibration study was done to determine whether the equipment could give a similar result to the literature. After several repeated experiments, the results showed approximately 7.7 $\pm 1 \%$ of nitrogen.

Although many previous studies have been made on reverse micelle systems using UV-Vis spectrophotometry for analytical purposes, which is the method preferred when analyzing for molecules that have conjugated $\pi-\pi$ bonds, this method cannot be used for determination of penicillin $G$ concentration because of its chemical structure. The penicillin $\mathrm{G}$ molecule has many $\mathrm{C}=\mathrm{O}$ bonds, which are weak bonds that make it difficult to absorb any particular wavelength using UV-Vis.

Equation (3.3) was used to calculate the percentage of nitrogen content $(\% N)$ after the extraction process and from these results the penicillin $\mathrm{G}$ content was obtained.

$$
\% N=\frac{14.01\left(V_{\text {titrant }}-V_{\text {titrant.blank }}\right) M_{\text {acid }}}{10\left(\rho_{w} V_{a q . f}\right)}
$$

where $V_{\text {titrant }}$ is the volume of titrant $(\mathrm{ml}), V_{\text {titrant.blank }}$ the volume of titrant blank (ml), $M_{\text {acid }}$ the molarity of acid $(\mathrm{mol} / \mathrm{l}), \rho_{w}$ the density water $(\mathrm{g} / \mathrm{ml})$, and $V_{\text {aq.f }}$ the final volume of the aqueous phase $(\mathrm{ml})$. There is no need to prepare a calibration curve since a blank can be used as a control for every experiment. However, from the viewpoint of testing the reliability of the Kjeldahl method for penicillin $G$, a calibration curve was obtained through several repeated experiments. Figure 3.10 shows a calibration curve of penicillin $\mathrm{G}$ for a concentration range from zero to 3.6 g/l versus the weight of nitrogen in the sample. The results indicate that for every concentration used, the percentage of nitrogen is approximately the same as that calculated from the molecular structure of penicillin $G$ (see Section 3.6.2). 
Therefore, the data from calibration curve study show that the Kjeldahl method is able to produce very reliable results for the current study.

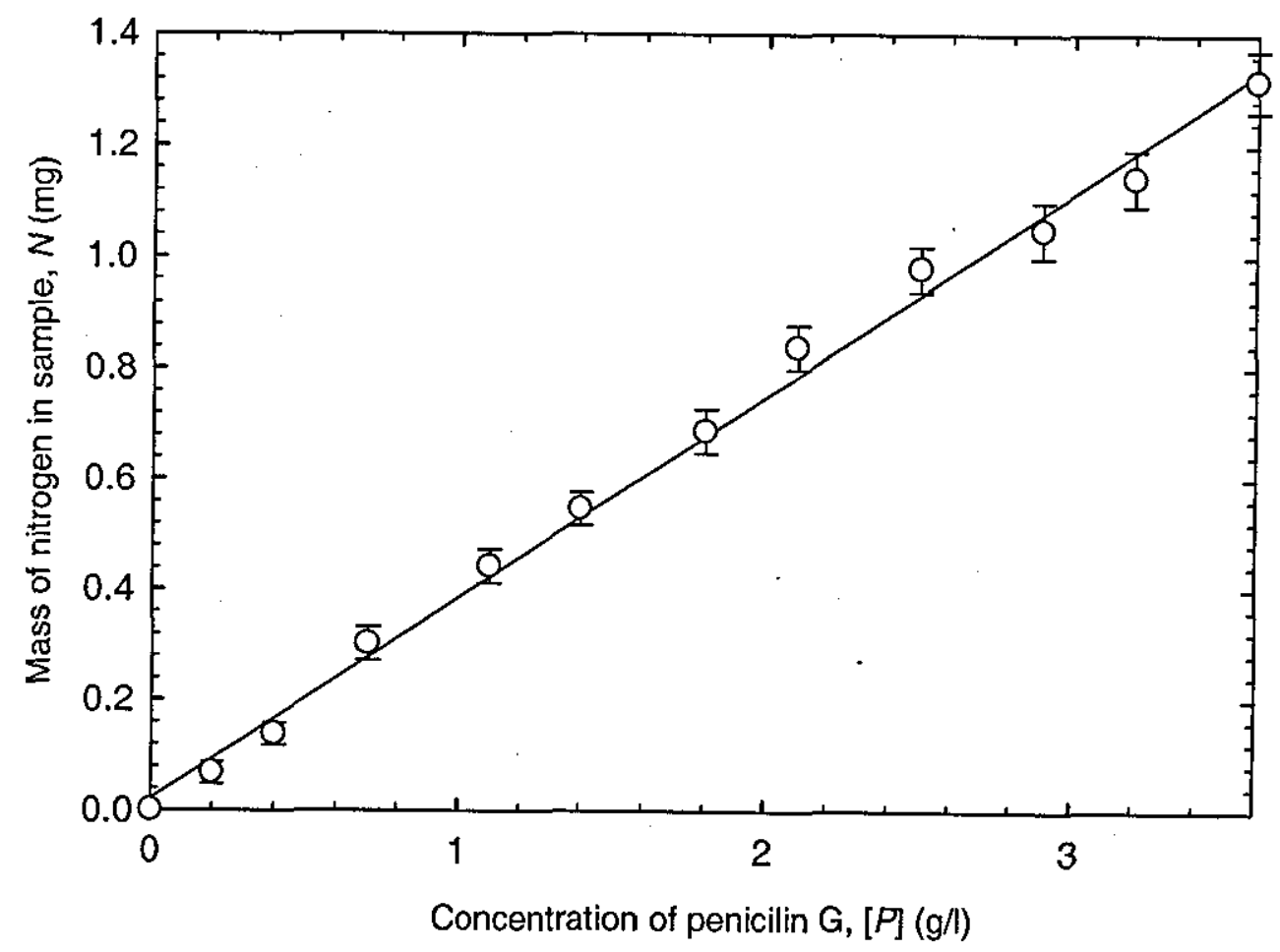

Figure 3.10: Calibration curve of penicillin G. Experiment conditions: $[\mathrm{KCl}]=0.1 \mathrm{M}$; temperature, $23 \pm 1^{\circ} \mathrm{C}$; stirring speed $=400 \mathrm{rpm} ; \mathrm{pH}=5.5$.

\subsubsection{Calculating Penicillin G Content}

After the extraction process, both aqueous and organic phases were separated carefully before analysis using the nitrogen analyser was carried out. The aqueous phase solutions were analysed and the nitrogen content obtained after the titration method was used to indicate the penicillin $G$ left in the aqueous phase after the extraction process.

The $\% N$ value from equation 3.3 is used to calculate the final mass of penicillin $\mathrm{G}$ in the aqueous phase (in $\mathrm{mg}$ ), $P_{a q . f}$, is:

$$
P_{a q . f}=\% N \rho_{w} V_{a q . f}\left(\frac{1}{100}\right) 1000
$$


where $\rho_{w}$ is the density of water, $(\mathrm{g} / \mathrm{ml})$ and $V_{a q . f}$ the final volume of the aqueous phase, (ml).

Therefore, the final concentration of penicillin $\mathrm{G}$ in the aqueous phase, $[P]_{a q . f}$, is:

$$
[P]_{a q . f}=\frac{P_{a q . f}}{V_{a q . f}}
$$

The final mass of penicillin $\mathrm{G}$ in the reverse micelle, $[P]_{r m+o . f}$ is calculated by adding together the mass of penicillin $G$ attached to surfactant and the mass of penicillin $G$ in the water transfer. A mass balance on penicillin $\mathrm{G}$ in the system is;

$$
V_{a q . i}[P]_{a q . i}=V_{r m+o . f}[P]_{a q . i}+S[P]_{s . f}+V_{a q . f}[P]_{a q . f}
$$

where $V_{a q . i}$ is the initial volume of water in aqueous phase, $(\mathrm{ml}),[P]_{\text {aq. } i}$ the initial concentration of penicillin $\mathrm{G}$ in the aqueous phase, ( $\mathrm{mg}$ penicillin/ml water), $V_{r m+o . f}$ the final volume of water in the reverse micelle, $(\mathrm{ml}), S$ the mass of surfactant used, (ml), $[P]_{s . f}$ the final concentration of penicillin $\mathrm{G}$ attached to the surfactant, (mg penicillin/ml surfactant), $V_{\text {aqf. }}$ the final volume of water in the aqueous phase, $(\mathrm{ml})$ and $[P]_{a q . f}$ the final concentration of penicillin $\mathrm{G}$ in the aqueous phase, (mg penicillin/ml water).

Rearranging this equation gives:

$$
S[P]_{s . f}=V_{a q . i}[P]_{a q . i}-V_{r m+o . f}[P]_{a q . i}-V_{a q . f}[P]_{a q . f}
$$

where $S[P]_{s . f}$ is the mass of penicillin $\mathrm{G}$ attached to surfactant, (mg). $S[P]_{s . f}$ can also be calculated using equations $(3.8)-(3.11)$.

The mass of surfactant in the solution, $S$, is:

$$
S=[S] V_{s . i}
$$

The concentration of penicillin $\mathrm{G}$ attached to surfactant, $[P]_{s . f}$ is calculated from:

$$
S[P]_{s . f}=[P]_{s . f}[S] V_{s . i} \frac{1}{10^{3}} \frac{1}{\rho_{s}}
$$


where $[S]$ is the concentration of surfactant used, $(\mathrm{g} / \mathrm{l}), V_{s . i}$ the initial volume of surfactant in the reverse micelle, $(\mathrm{ml})$ and $\rho_{s}$ the density of surfactant, $(\mathrm{g} / \mathrm{ml})$.

Rearranging equation (3.6) gives:

$$
[P]_{s . f}=\frac{\left(S[P]_{s . f}\right) 10^{3} \rho_{s}}{[S] V_{s . i}}
$$

The mass of penicillin $\mathrm{G}$ attached to surfactant (in $\mathrm{mg}$ ), $P_{s . f}$, is:

$$
P_{s . f}=S[P]_{s . f}
$$

The mass of penicillin $\mathrm{G}$ in the water pool (in $\mathrm{mg}$ ), $P_{w p}$, is:

$$
P_{w p}=[P]_{a q . i} V_{w t}
$$

where $V_{w t}$ is the volume of water transfer, $(\mathrm{ml})$. Therefore, the final total mass of penicillin $\mathrm{G}$ in the reverse micelle phase, $[P]_{r m+o f}$, is:

$$
P_{r m+o . f}=P_{s . f}+P_{w p}
$$

and the final volume of the reverse micelle organic phase, $V_{r m+o f}$, is:

$$
V_{r m+o . f}=V_{w t}+V_{r m+o . i}
$$

where $V_{r m+o . i}$ is the initial volume of reverse micelle organic phase, (ml).

By dividing the final mass of penicillin $\mathrm{G}$ in the reverse micelle organic phase by the final volume of the reverse micelle organic phase, we obtain;

$$
[P]_{r m+o . f}=\frac{P_{r m+o . f}}{V_{r m+o . f}}
$$

\subsubsection{Conclusions}

In this chapter experiments on the solubilisation of penicillin $G$ from both aqueous and organic phase were presented. The materials and characterization techniques for the measurement of feed solution characteristics were discussed. The methods used were a Wilhelmy Ring technique for surface tension measurements, a Kjeldahl 
method for determination of the mass of penicillin $\mathrm{G}$ in the aqueous solution after extraction, a Karl Fisher titration for water content analysis, and a $\mathrm{pH}$ meter was used to determine $\mathrm{pH}$ of the aqueous solutions before and after the extraction process. A calibration curve and equations for determination of penicillin $G$ content in the aqueous phase after extraction process were also discussed. 


\section{CHAPTER FOUR \\ Results and Discussion - Forward Extraction}

\subsection{Introduction}

This work has focussed on two aspects:

- A study of the factors that influence penicillin $G$ transfer during forward extraction from an aqueous phase (containing $\mathrm{KCl}$ ) into a reverse micellar organic phase (containing AOT and isooctane)

- A study of the factors that encourage penicillin $G$ solubilised in the reverse micelle to be transferred into a fresh aqueous phase (backward extraction).

Both objectives have considered a thermodynamic framework of penicillin $G$ solubilisation. Chapter 4 focusses on the results obtained for forward extraction. Firstly, interfacial association and phase volume changes, partitioning of penicillin $G$ between the organic and aqueous phases, other effects on solubilisation of penicillin $\mathrm{G}$ in the reverse micelle, and reverse micelle formation and water content are discussed. Secondly, a thermodynamic framework for solubilisation during the forward process is presented through use of a Langmuir equation and a model proposed by Brandani et al. (1994). The results presented in this chapter are typical of those obtained; additional information can be found in Appendix 2.

\subsection{Sample Characterisation - Critical Micelle Concentration}

The critical micelle concentration (CMC), the lowest concentration of surfactant needed for reverse micelle formation, of the AOT in isooctane was measured using a tensiometer (as described in Section 3.3.2) and typical results are shown in Figure 4.1. For the experiments without penicillin, the AOT was mixed into a sample of 
isooctane and the surface tension of the mixture measured. For the experiments where penicillin was present, the penicillin was extracted from an aqueous solution and the organic and aqueous solutions separated before the surface tension of the organic phase was measured. The concentration of penicillin is therefore different for each data point, and corresponds to its equilibrium partitioning between the phases. At $[S]=0 \mathrm{~g} / \mathrm{l},[P]_{r m+o f f}=0 \mathrm{~g} / \mathrm{l},[P]_{r m+o . f}$ is observed to increase by increasing $[S]$ up to $88 \mathrm{~g} / \mathrm{l}$. As the surface tension reached a plateau when $[S]$ increased above $88 \mathrm{~g} / \mathrm{l},[P]_{r m+o f}$ also reached a plateau at $2.9 \mathrm{~g} / \mathrm{l}$.

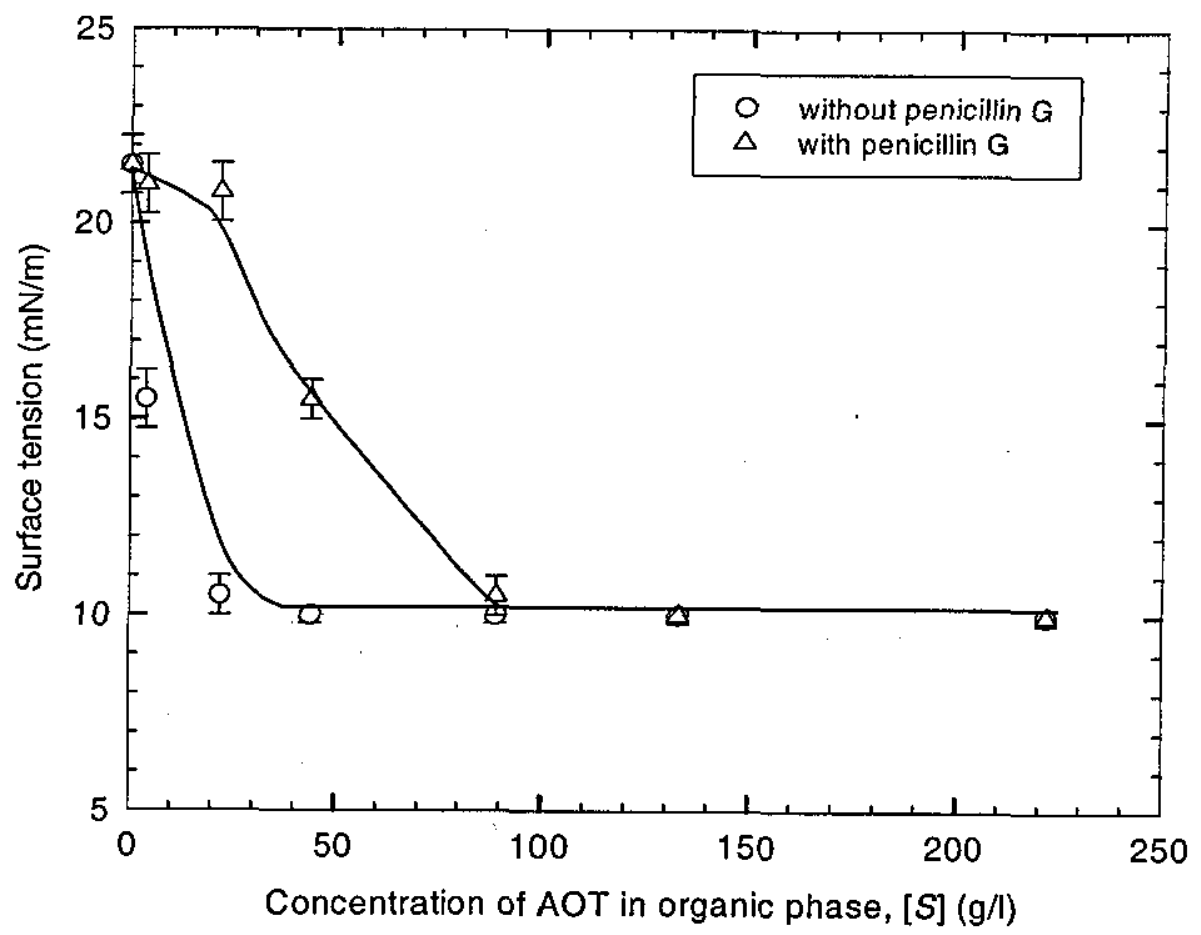

Figure 4.1: Effect of AOT concentration on the surface tension of isooctane with and without penicillin $\mathrm{G}$. (Initial penicillin in the aqueous phase, $3.6 \mathrm{~g} / \mathrm{l} ;[\mathrm{KCl}]=10 \mathrm{~g} / \mathrm{l}$; temperature, $23 \pm 1^{\circ} \mathrm{C}$; stirring speed, 400 rpm for 10 minutes; $\mathrm{pH} 7.6 \pm 0.5$ ).

Figure 4.1 shows that the $\mathrm{CMC}$ of the solution containing penicillin $\mathrm{G}$ is at $[S] \approx 100$ $\mathrm{g} / \mathrm{l}$, which is higher than the value without penicillin $\mathrm{G}$ (at $[S] \approx 25 \mathrm{~g} / \mathrm{l}$ ). The CMC value is higher for the system with penicillin $G$ because the molecules that solubilised in the reverse micelle acted to increased the surface tension of the AOT solutions. 
Use of a stirrer speed greater than $400 \mathrm{rpm}$ caused formation of an emulsion that made clear phase separation difficult. In some cases the emulsion was short lived and disappeared after 24 hours settling time. The effect of the settling period on the concentration of penicillin $\mathrm{G}$ in the organic phase at different AOT concentrations was studied. From our observations there were no significant differences between 24 hours and one week settling times indicating that the penicillin solubilisation had reached the equilibrium state; more than 24 hours settling time did not have an effect on the extraction efficiency.

\subsection{Interfacial Association and Phase Volume Changes}

Penicillin $G$ solubilisation into the reverse micelle organic phase is influenced by parameters such as surfactant concentrations that are related to the organic phase, in addition to the condition of the aqueous phase (such as $\mathrm{pH}$ and salt concentration). The motivation for using AOT surfactant in this study is because of its ease of forming reverse micelle (Krieger et al., 1997; Naoe et al., 1999), its stability in comparison with other systems (Krieger et al., 1997) and the fact that it has been used in many published studies of other systems with success.

At equilibrium, the penicillin is distributed between four solubilisation sites: (i) the aqueous phase continuum, (ii) the water pools lying within the reverse micelle in the organic continuum (see Figure 4.2), (iii) the organic continuum, and (iv) at the reverse micelle interface between the organic phase and the water pool. When experiments were carried out using different AOT concentrations, there was an increase in the volume of the organic phase that was proportionate to a decrease of the aqueous phase volume. The additional volume of the organic phase, shown in Figures 4.3 and 4.4 , increased when concentrations of AOT $>40 \mathrm{~g} / \mathrm{l}$ were used. 


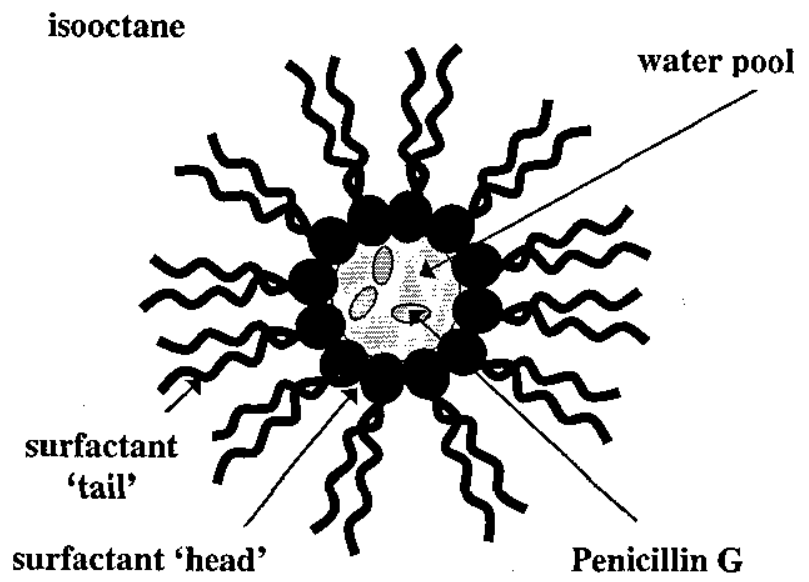

Figure 4.2: Schematic diagram of the reverse micelle phase. The penicillin is entrapped in a water pool formed inside the reverse micelle, which itself lies in the organic (isooctane) continuous phase.

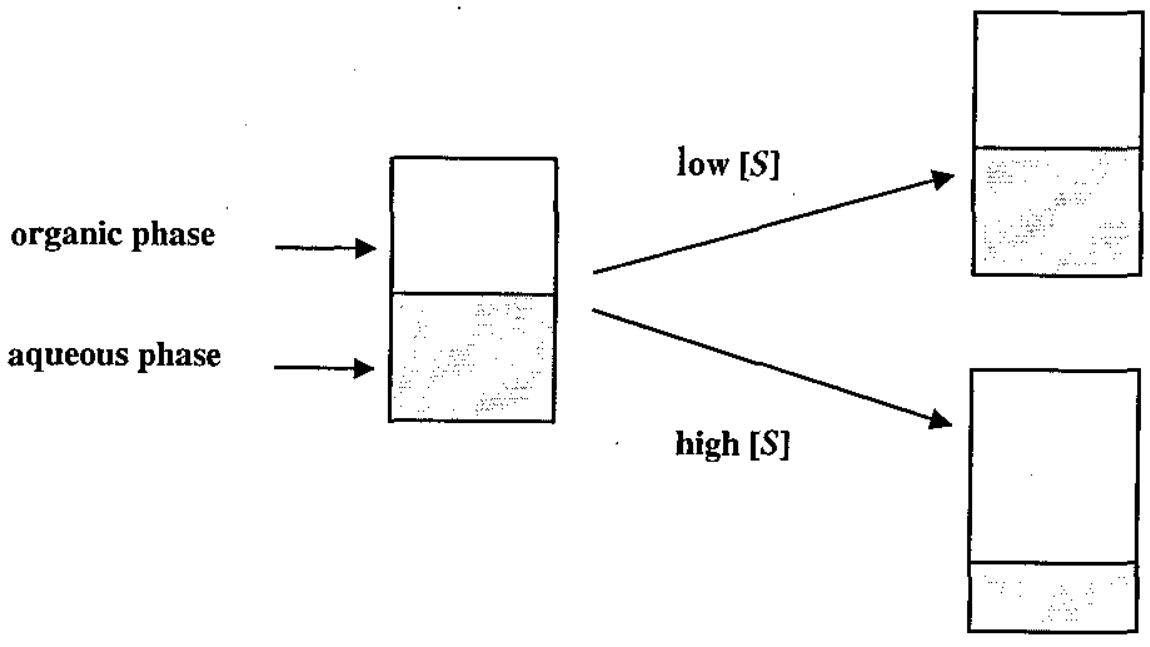

Figure 4.3: Phase sequence after 1 week. $\left([\mathrm{KCl}]=10 \mathrm{~g} / \mathrm{l}\right.$; temperature, $23 \pm 1{ }^{\circ} \mathrm{C}$; stirring speed, 400 rpm for 10 minutes; $\mathrm{pH} 7.6 \pm 0.5$; initial penicillin $\mathrm{G}$ concentration in the aqueous phase, $3.6 \mathrm{~g} / \mathrm{l})$. 


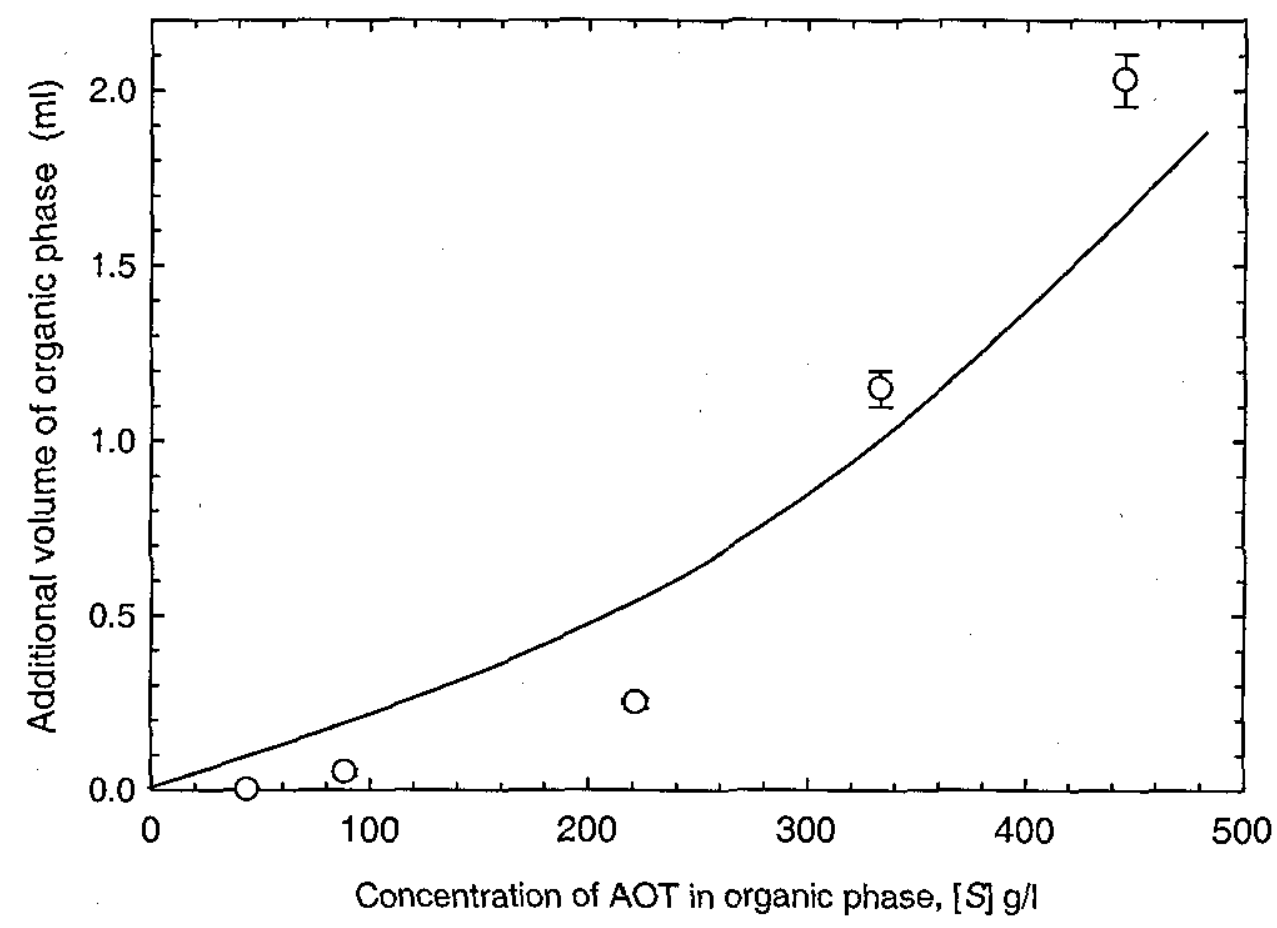

Figure 4.4: Additional volume of the organic phase at different AOT concentrations. (Initial volumes of the organic and aqueous phases were each $5 \mathrm{ml} ;[\mathrm{KCl}]=10 \mathrm{~g} / \mathrm{l}$; temperature, $23 \pm 1^{\circ} \mathrm{C}$; stirring speed, $400 \mathrm{rpm}$ for 10 minutes; $\mathrm{pH} 7.6 \pm 0.5$; initial penicillin $\mathrm{G}$ concentration in the aqueous phase, $3.6 \mathrm{~g} / \mathrm{l}$ ).

At AOT concentrations less than the $\mathrm{CMC}$, there was no visible increase in the volume of the organic phase. When the AOT concentration was increased to above about $40 \mathrm{~g} / \mathrm{l}$, the effect of reverse micelle formation showed itself by an increase in the volume of the organic phase as water was transferred into the reverse micellar structures.

Leodidis and Hatton (1990) suggested, based on arguments stemming from Gibbs free energy of transfer of a solute from water to the surfactant interface, that an interfacial distribution coefficient could be calculated on the basis of solute concentration measurements of the initial and final aqueous phases. For a solute that does not associate with the reverse micellar interface $[P]_{\text {aq. }}=[P]_{\text {aq.i }}$, and deviations from this line imply interfacial association of the solute. Figure 4.5 is a plot of the $[P]_{\text {aq.f }}$ versus $[P]_{\text {aq. } i}$ data for the AOT-penicillin system. 


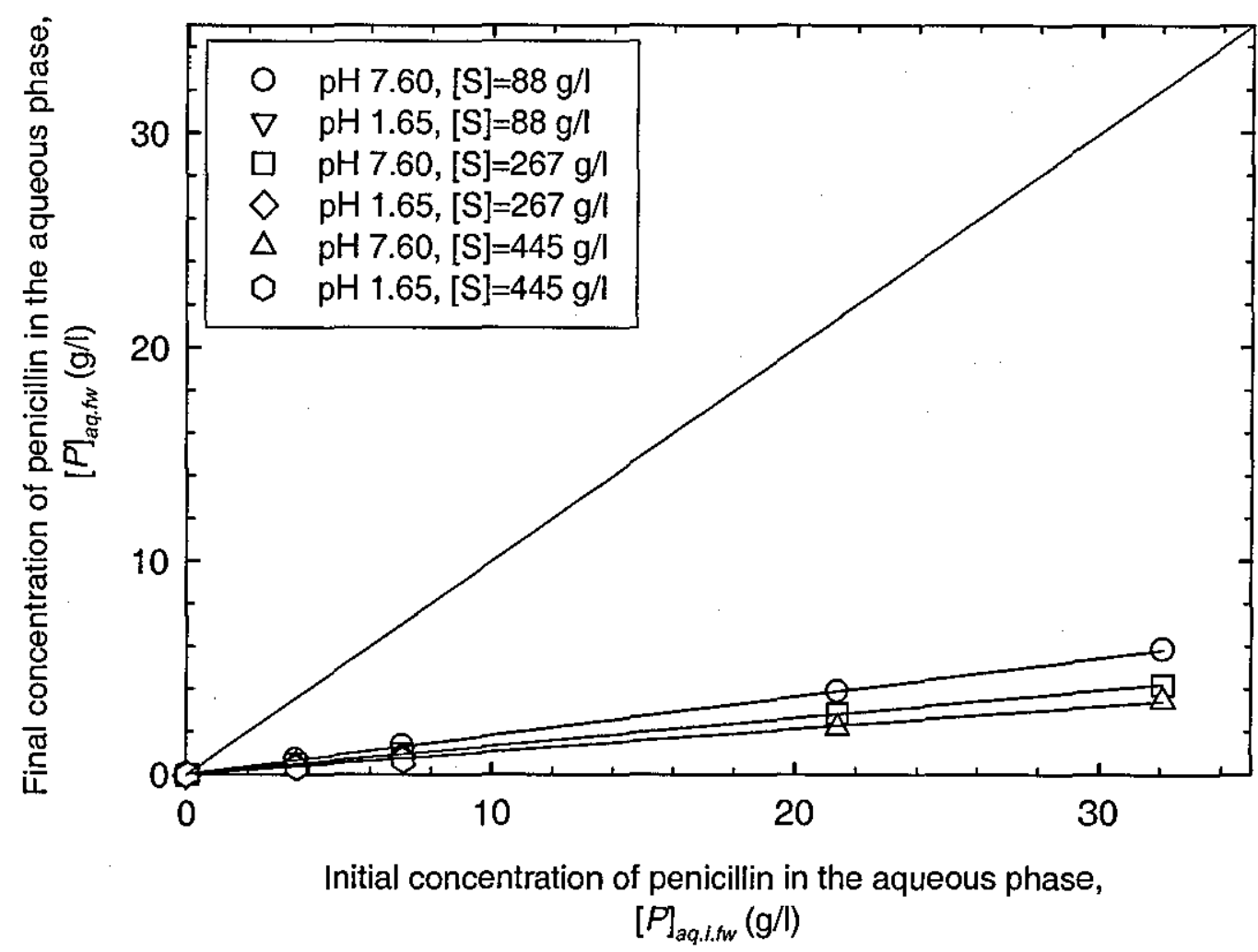

Figure 4.5: Final penicillin $\mathrm{G}$ concentration in the aqueous phase plotted against the initial penicillin $\mathrm{G}$ concentration before extraction at $23^{\circ} \mathrm{C}$ and $[\mathrm{KCl}]=10 \mathrm{~g} / \mathrm{l}$.

Figure 4.5 illustrates that penicillin $\mathrm{G}$ is an interfacially active molecule at every tested AOT concentration and at $\mathrm{pH}$ values of 1.65 and 7.6. These $\mathrm{pH}$ values were used due to the high solubilisation of penicillin $\mathrm{G}$ at both $\mathrm{pHs}$ (also discussed further in Section 4.4). The slope of the data points indicates there is the least surface activity at an AOT concentration of $88 \mathrm{~g} / \mathrm{l}$; at an AOT concentration of $445 \mathrm{~g} / 1$ the deviation of the data is furthest from the $45^{\circ}$ line, indicating greatest surface activity.

Leodidis and Hatton (1990) also showed that an interfacially active molecule can be identified through a plot of molecule transfer (in their case amino acid) $\left(t_{a}\right)$ versus the fractional water transfer $\left(t_{w}\right)$ to the organic phase. Mass balances show that $t_{a}$ and $t_{w}$ are given by:

$$
t_{w}=1-\frac{V_{a q . f}}{V_{a q . i}}
$$




$$
t_{a}=1-\frac{[P]_{a q . f} V_{a q . f}}{[P]_{a q . i} V_{a q . i}}
$$

$t_{w}$ can be varied by changing the $\mathrm{pH}$ or the salt content of the aqueous phase or the AOT concentration in the initial organic phase. The penicillin transfer $\left(t_{a}\right)$ is plotted against the fractional water transfer $\left(t_{w}\right)$ in Figure 4.6, which gives further support to penicillin being interfacially active in the presence of AOT.

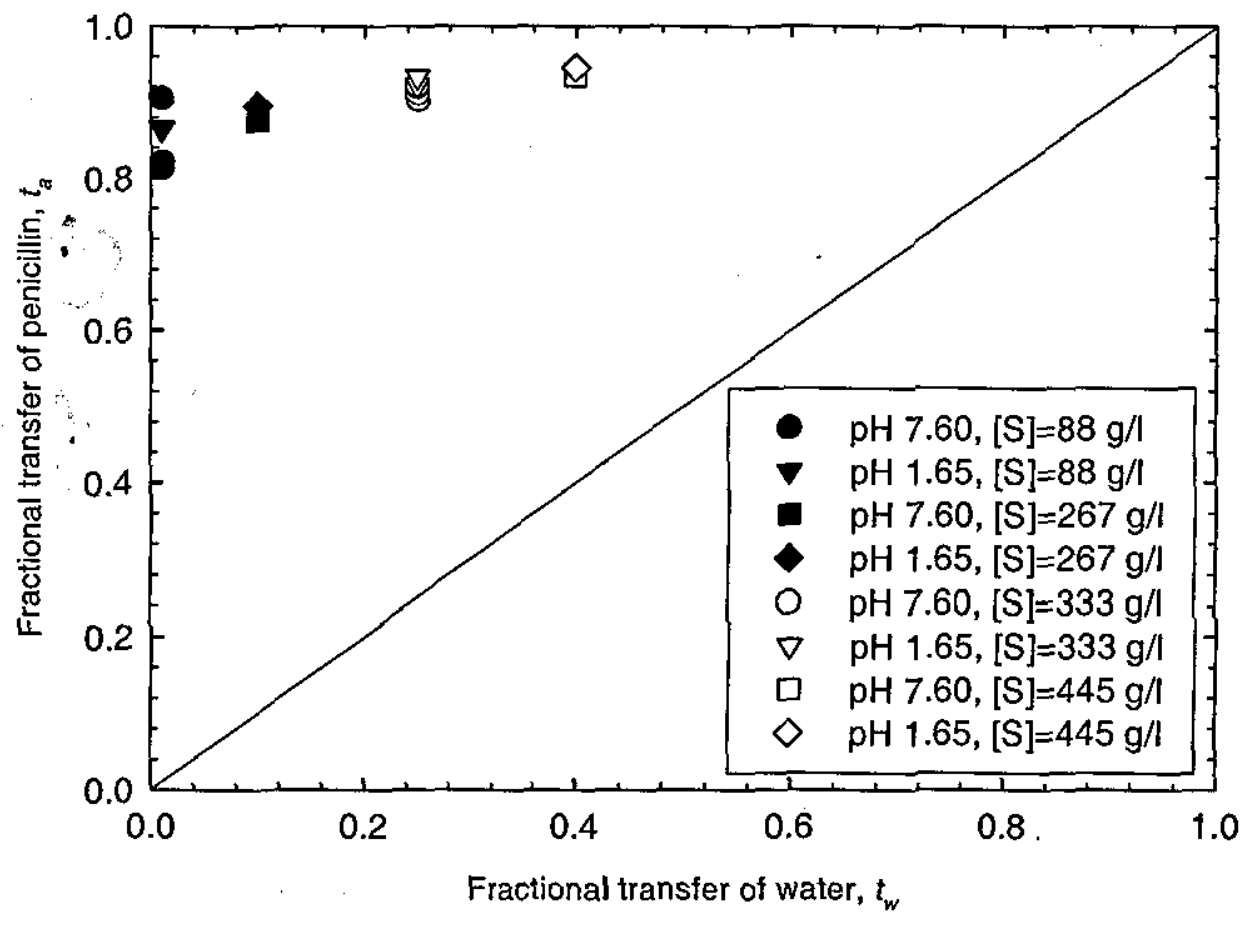

Figure 4.6: Fractional transfer of penicillin $\mathrm{G}$ plotted against the fractional water transfer to the reverse micelle organic phase at different $\mathrm{pH}$ values and AOT concentrations.

$t_{w}$ indicates the increase in organic phase volume and when $t_{w}$ is finite $V_{a q . f}<V_{a q . i}$; further, from equations (4.1) and (4.2), when $t_{a}>t_{w}$ and $[P]_{a q . f}<[P]_{a q . i}$.

$$
t_{a}-t_{w}=\left(1-t_{w}\right)\left(1-\frac{[P]_{a q . f}}{[P]_{a q . i}}\right)
$$

The vertical displacement of the experimental data from the $45^{\circ}$ line is a measure of the strength of interfacial association; Figure 4.6 shows that the interfacial 
association is dependent on both $\mathrm{pH}$ and surfactant concentration. Similar trends were observed for the AOT-amino acid system by Leodidis and Hatton (1990).

\subsection{Partitioning of Penicillin $G$ between Phases}

Initially, the effect of AOT concentration on reverse micellar extraction was examined using an aqueous solution at $\mathrm{pH} 7.6$ and $[\mathrm{KCl}]=7 \mathrm{~g} / \mathrm{l} . \quad \mathrm{A}$ 'white precipitate' was observed at the interface of some samples when the saltt concentration used was less than $7 \mathrm{~g} / \mathrm{l}$; later experiments showed that the onset of precipitate formation was dependent on the ratio of penicillin concentration in the aqueous phase to surfactant concentration $[S]$. Some researchers extracting proteins found that using AOT concentrations greater than $7 \mathrm{~g} / \mathrm{l}$ caused interfacial protein precipitation (e.g., Dekker et al., 1989). The 'white precipitate' is a dispersion phenomenon that occurred when the ratio $[P]_{a q} /[S]$ was high because of low hydrophobicity in the system due to the low surfactant concentration used, which appears to be similar to protein aggregation rather than protein solubilisation into the organic phase found by others (Kyung and Jee, 2005).

A sought after effect of the AOT concentration on penicillin extraction is to increase the mass of penicillin transferred to the reverse micellar phase. It is known that the surfactant concentration controls the number of reverse micelle and hence the capacity to solubilise biomolecules into the reverse micelle pool (Pires et al., 1996; Jun et al., 2004). Increasing the surfactant concentration increases the number of reverse micelle (Yu et al., 2003), thus leading to an improvement in extraction capacity of the reverse micelle. Figure 4.7 shows that as the concentration of AOT is increased from 0 to $88 \mathrm{~g} / \mathrm{l}$, so the mass of penicillin extracted into the reverse micellar phase increased.

The mass of penicillin in the reverse micelle levels off when the AOT concentration is varied from $88 \mathrm{~g} / 1$ to $222 \mathrm{~g} / 1$. However, the concentration of penicillin $\mathrm{G}$ in the reverse micelle then decreases when the AOT concentration is further increased from $222 \mathrm{~g} / \mathrm{l}$ to $445 \mathrm{~g} / \mathrm{l}$. There is an optimum surfactant concentration observed between 
$88 \mathrm{~g} / \mathrm{l}$ and $222 \mathrm{~g} / \mathrm{l}$, which corresponds to the maximum limit of penicillin $\mathrm{G}$ transfer into the reverse micelle as shown in Figure 4.7.

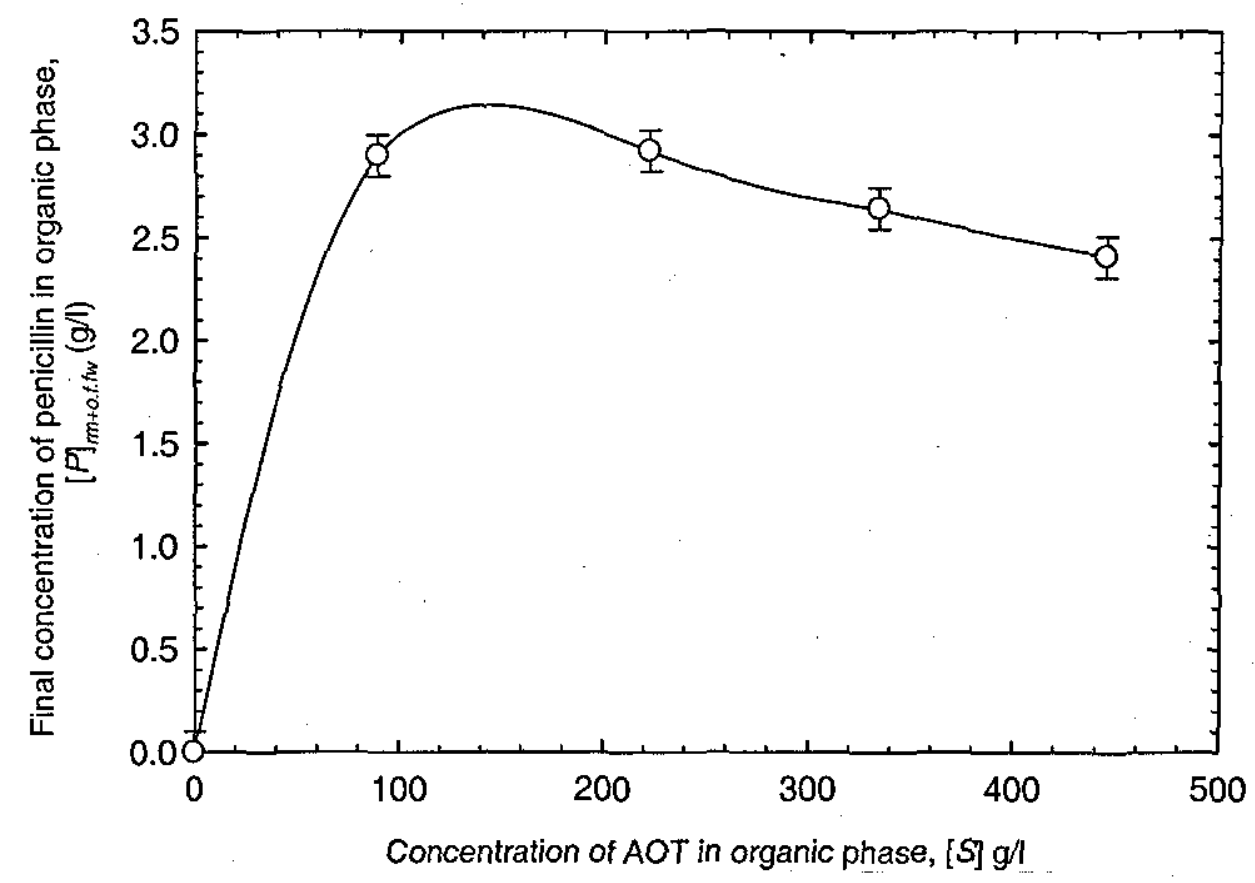

Figure 4.7: Concentration of penicillin $\mathrm{G}$ in the organic phase at different AOT concentrations. $\left([\mathrm{KCl}]=10 \mathrm{~g} / 1\right.$; temperature, $23 \pm 1^{\circ} \mathrm{C}$; stirring speed, $400 \mathrm{rpm}$ for 10 minutes; $\mathrm{pH} 7.6 \pm 0.5$; initial penicillin $\mathrm{G}$ concentration in the aqueous phase $3.6 \mathrm{~g} / \mathrm{l}$ ).

Penicillin $\mathrm{G}$ is a small molecule with a molecular weight of $356 \mathrm{~g} / \mathrm{mol}$; according to Pires et al. (1996), small sized molecules are more easily extracted to the reverse micelle using low surfactant concentration. From the experimental work, use of AOT concentration $>445 \mathrm{~g} / \mathrm{l}$ produced an emulsion that was stable (when settled under gravity), making it difficult to separate and analyse.

It was shown experimentally that penicillin $\mathrm{G}$ did not solubilise in the organic phase without AOT. Since penicillin G was extracted into the organic phase only when reverse micelle were present, it is assumed that the penicillin $G$ is located within their structure. Hence, the distribution coefficient for forward extraction $\left(K_{f w}\right)$ is defined by: 


$$
K_{f w}=\frac{[P]_{m+o . f . f w}}{[P]_{a g . f . f w}}
$$

Where $[P]_{r m+o . f f w}$ is the final concentration of penicillin $\mathrm{G}$ in the reverse micelle organic phase for forward extraction, $(\mathrm{g} / \mathrm{l})$, and $[P]_{\text {aq.ffiv }}$ the final concentration of penicillin $\mathrm{G}$ in the aqueous phase for forward extraction, $(\mathrm{g} / \mathrm{l})$. A higher value of the distribution coefficient indicates better solubilisation of penicillin $G$ into the reverse micelle.

Figure 4.8 shows that the distribution coefficient increases with increasing AOT concentration. The extent of solubilisation of penicillin into the reverse micellar phase depends on the quantity of reverse micelle, which during mixing are better dispersed in the bulk aqueous phase when more surfactant molecules are present. Haghtalab and Osfouri (2003) asserted that adding more surfactant to the solution intensifies the interaction between the reverse micelle and the protein molecules (in their case) and results in the number of moles, and the maximum mole number of protein on the surface of the reverse micelle, being changed. Figure 4.8 shows that there is no saturation limit for the concentration of penicillin (up to $[P]_{r m+o . f f w}=30$ $\mathrm{g} / \mathrm{l})$ that can be transferred into the reverse micellar phase.

However, there is a concentration limit of AOT to effect solubilisation of a maximum concentration of penicillin $G$ into reverse micelle. The distribution coefficients of penicillin $G$ are initially identified at $[S]=267 \mathrm{~g} / 1$ and $[S]=445 \mathrm{~g} / 1$. This trend confirmed that penicillin $G$ is an interfacially active compound which can be readily transferred to the reverse micelle. 


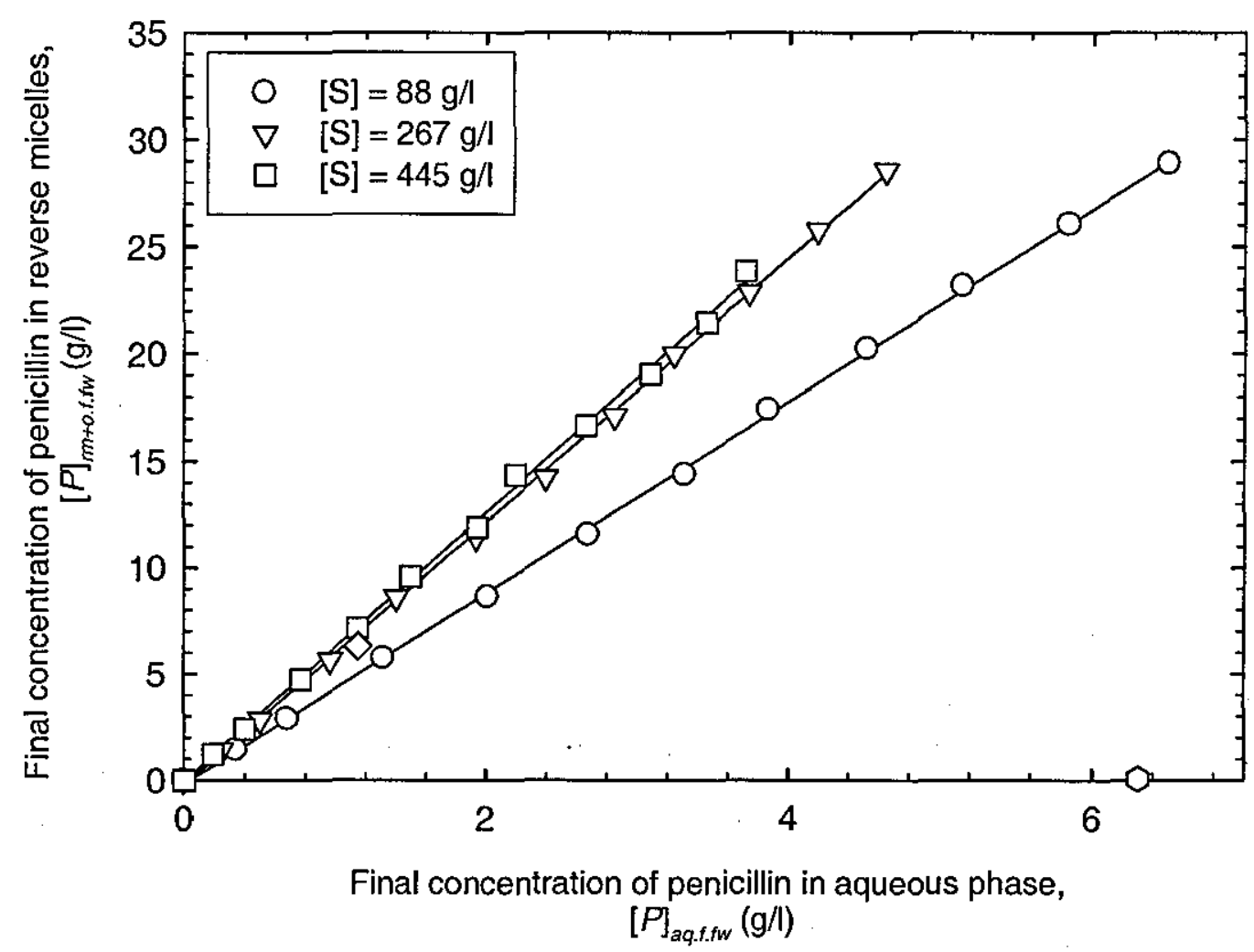

Figure 4.8: Distribution of penicillin $\mathrm{G}$ between the reverse micelles in the organic phase, $[P]_{r m+o f f w}$, and the aqueous phase, $[P]_{a q . f f w^{*}}\left([\mathrm{KCl}]=10 \mathrm{~g} / 1\right.$; temperature, $23 \pm 1^{\circ} \mathrm{C}$; stirring speed, $400 \mathrm{rpm}$ for 10 minutes; $\mathrm{pH} 7.6 \pm 0.5$; initial penicillin $\mathrm{G}$ concentration in the aqueous phase $3.6 \mathrm{~g} / \mathrm{l})$.

At higher initial concentrations of penicillin in the aqueous phase the ion-dipole interaction between penicillin and AOT molecules are larger than the ion-ion interaction between penicillin molecules. In previous studies of protein and amino acid extraction using reverse micelle systems, other researchers buffered the aqueous phase to adjust $\mathrm{pH}$ (Goklen and Hatton, 1987; Leodidis and Hatton, 1990; Jolivalt et al., 1993). However, Wang et al. (1995) pointed out that buffers may interfere with the solubilisation of biomolecules into the reverse micellar phase because the buffer anions can compete with the amino acid (in their case) to interact with the surfactant headgroup; in other work, buffers were avoided because over such a wide range of $\mathrm{pH}$ values they would give the addition of an unnecessary mixture of ions to the system (Andrews, 1993). In the current study $\mathrm{NaOH}$ and $\mathrm{HCl}$ have been used to adjust $\mathrm{pH}$. To examine the effect of $\mathrm{pH}$ on the partitioning of penicillin, aqueous $\mathrm{KCl}$ solutions containing $3.6 \mathrm{~g} / \mathrm{l}$ of penicillin and various $\mathrm{pH}$ values were contacted 
with solutions of AOT of various concentrations at a volume ratio of 1:1. The results are shown in Figure 4.9 as a relation between the concentration of penicillin $G$ in the organic phase and the $\mathrm{pH}$ value of the aqueous phase at the equilibrium state.

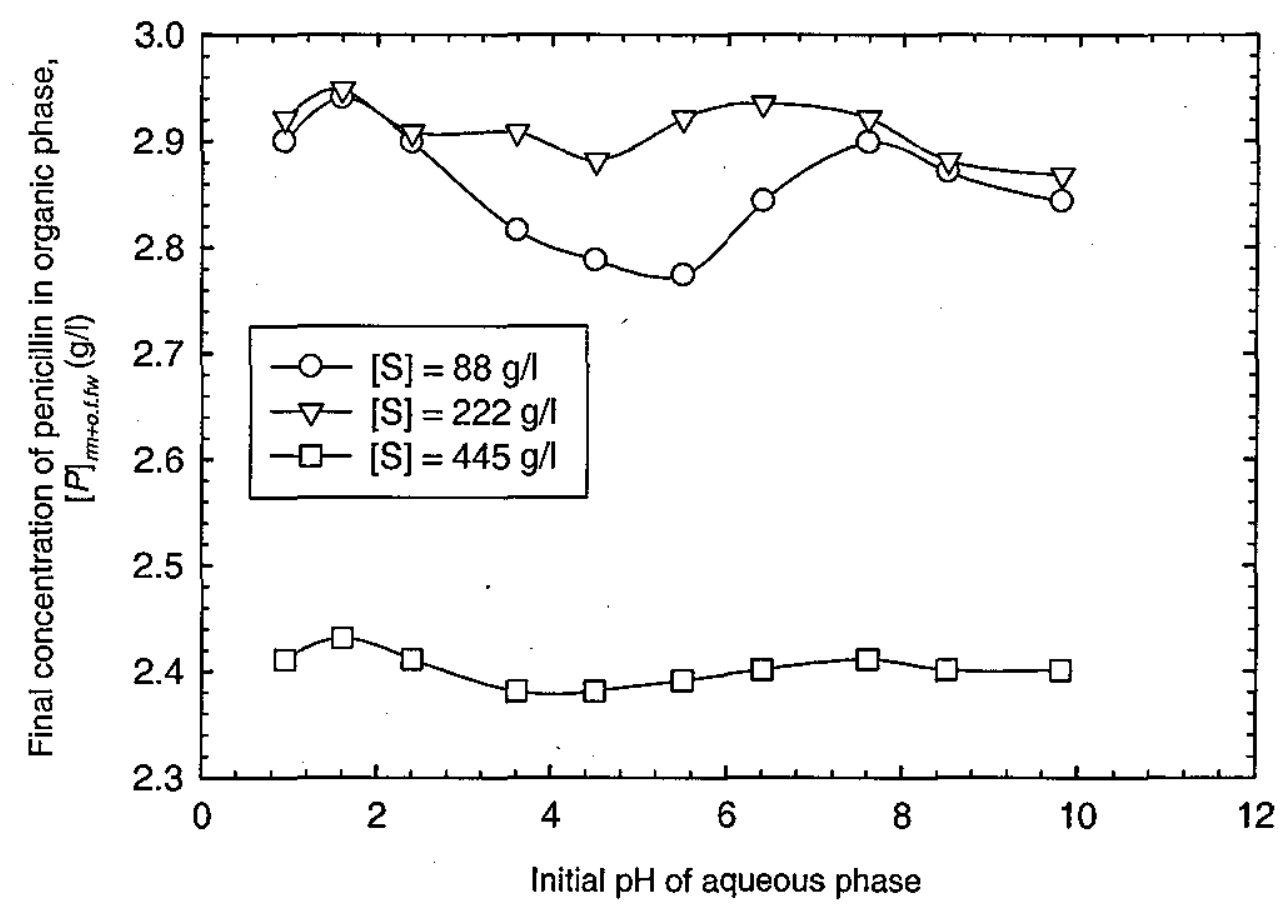

Figure 4.9: Effects of $\mathrm{pH}$ and AOT concentration on the mass of penicillin $\mathrm{G}$ extracted into the reverse micelle phase. (Temperature, $23 \pm 1^{\circ} \mathrm{C}$; stirring speed, $400 \mathrm{rpm}$ for 10 minutes; initial penicillin $\mathrm{G}$ concentration in the aqueous phase, $3.6 \mathrm{~g} / \mathrm{l}$ ).

From Figure 4.9, the results obtained show there are maximum penicillin G concentration in the reverse micellar phase at moderate $\mathrm{pH}$ value i.e. 7.6 and also at low $\mathrm{pH}$ values (as low as 1.6); this could be attributed to the high electrostatic attraction between AOT anions and the positively charged penicillin $\mathrm{G}$ aggregates at such low $\mathrm{pH}$. Previous studies on protein extraction have also shown that $\mathrm{pH}$ plays important role in reverse micellar systems (Nishiki et al., 1993; Ono et al., 1996; Hu and Gulari, 1996; Pires et al., 1996; Tzeng et al., 1999; Kilikian et al., 2000; Yu et $a l ., 2003)$. Noble and Varley (1999), using protein as the biomolecule, pointed out that the $\mathrm{pH}$ of the protein and surfactant solutions could be manipulated so that the protein would exhibit a net charge opposite to that of the surfactant, thus allowing electrostatic interactions between the protein and surfactant in the different phases. Kilikian et al. (2000) explained that the overall protein charge is determined by the 
$\mathrm{pH}$ of the aqueous phase and the isoelectric point ( $\mathrm{pI}$ ) of the biomolecule used (protein, in their case). If the $\mathrm{pH}$ of the aqueous phase is higher than the $\mathrm{pI}$, the charge is negative, but if the $\mathrm{pH}$ is lower than the $\mathrm{pI}$, the charge is positive. Using this postulate for the anionic AOT, the penicillin $\mathrm{G}(\mathrm{pI}=2.74)$ is expected to have strong attraction with the surfactant head group at $\mathrm{pH}<2.74$. Hence, more penicillin $\mathrm{G}$ could be solubilized into the reverse micellar solution at $\mathrm{pH}<\mathrm{pl}$. From the viewpoint of industrial production of penicillin $\mathrm{G}$, extraction is carried out at $\mathrm{pH} 2.5$ 3.0 .

Figure 4.9 shows that at $\mathrm{pH}>\mathrm{pI}$ a higher AOT concentration needs to be used in order to get the same concentration of penicillin $\mathrm{G}$ in the organic phase as at $\mathrm{pH}<\mathrm{pI}$; however it should note that there is an optimum AOT concentration (which is $222 \mathrm{~g} / \mathrm{l}$ for the range of experiments in this work). The concentration of penicillin $G$ in reverse micelle decreases dramatically when $[S]=445 \mathrm{~g} / 1$ is used. This raises the question of whether the $\mathrm{pH}$ can be manipulated to get better penicillin $\mathrm{G}$ extraction at low AOT concentration in an industrial application (it should be noted that penicillin broths are compositionally more complex than those used in this work, which will change the equilibria that can be established in the extraction process). The use of a low surfactant concentration would be helpful because amphiphilic molecules are difficult to remove in subsequent downstream processing (Goto et al., 1998).

Ono et al. (1996), using anionic surfactant dioleyl phosphoric acid (DOLPA) to extract haemoglobin $(\mathrm{pI}=6.8$ ) found that even though the best extraction was obtained at $\mathrm{pH}<\mathrm{pI}(5.5-6.5)$, a precipitate occurred at $\mathrm{pH}<5$. They found that at the $\mathrm{pH} \ll<\mathrm{pI}$, the biomolecules tended to form a complex that binds to the surfactant. In the current study, precipitation was observed when higher initial penicillin $G$ concentrations were used at low $\mathrm{pH}$. Although an extremely low $\mathrm{pH}$ was used, at $[\mathrm{KCl}]=10 \mathrm{~g} / \mathrm{l}$ and $400 \mathrm{rpm}$ stirring speed, a transparent two phase system was obtained with a clear interphase when $[P]_{a q . i}<10 \mathrm{~g} / \mathrm{l}$. From the results, it appears that the $\mathrm{pH}$ effect is important for penicillin $\mathrm{G}$ extraction, although the electrostatic contribution is also relevant for the AOT reverse micelle which achieved better binding at higher $\mathrm{pH}$ values. 


\subsection{Other Effects on the Solubilisation of Penicillin G}

\subsubsection{Salt Concentration}

The salt concentration of the aqueous phase influences the solubilisation of penicillin into the reverse micellar phase. The salt concentration was investigated by varying the $\mathrm{KCl}$ concentration in the aqueous phase at fixed $\mathrm{pH} 7.6$ (Figure 4.10). When $[\mathrm{KCl}]>10 \mathrm{~g} / \mathrm{l}$, the penicillin concentration in the organic phase was found to decrease, although increasing $[\mathrm{KCl}]$ from 0 to $10 \mathrm{~g} / 1$ also showed a significant increase of penicillin in the organic phase. A decrease of the volume of water in the micellar phase was found to accompany an increase of [ $\mathrm{KCl}]$.

At $[\mathrm{KCl}]<5 \mathrm{~g} / \mathrm{l}$ almost all of the surfactant migrated to the aqueous phase and no reverse micelle were formed in the organic phase. This phenomenon was observed visually, and made addition of salt to the aqueous penicillin $G$ solution very important to avoid the formation of a stable emulsion. A minimum amount of salt is necessary to 'salt out' the surfactant from the excess aqueous phase into the organic phase and form reverse micelle (Wang et al., 1994; Rabie et al., 1997). Reverse micelle will not form if there is too little salt in the aqueous phase; however, if there is too much salt, the efficiency of surfactant molecules to solubilise the penicillin $\mathrm{G}$ molecules reduced. Wang et al. (1995) reported that addition of salt is required to reduce electrostatic repulsion between the surfactant head groups. They supposed that if there is no salt added to the system, the aggregation of surfactant and/or the surfactant and (the tested) protein complex form a coagulum which induces a phase separation problem.

In the current work, the solubilisation of water by an AOT reverse micellar solution in equilibrium with an aqueous phase (both with and without penicillin $G$ ) was found to be a strong function of salt concentration. Others have suggested that decreases of both biomolecule concentration and water content in the organic phase happen because of a size exclusion effect that occurrs with the decrease in micelle size. Krieger et al. (1997) and Aires-Barros and Cabral (1991) asserted that a decrease in the solubilisation of the enzyme with increases in ionic strength are a consequence of smaller micelles being formed because of the reduced interaction between the surfactant polar head group and the hydrophilic region of the enzyme. 


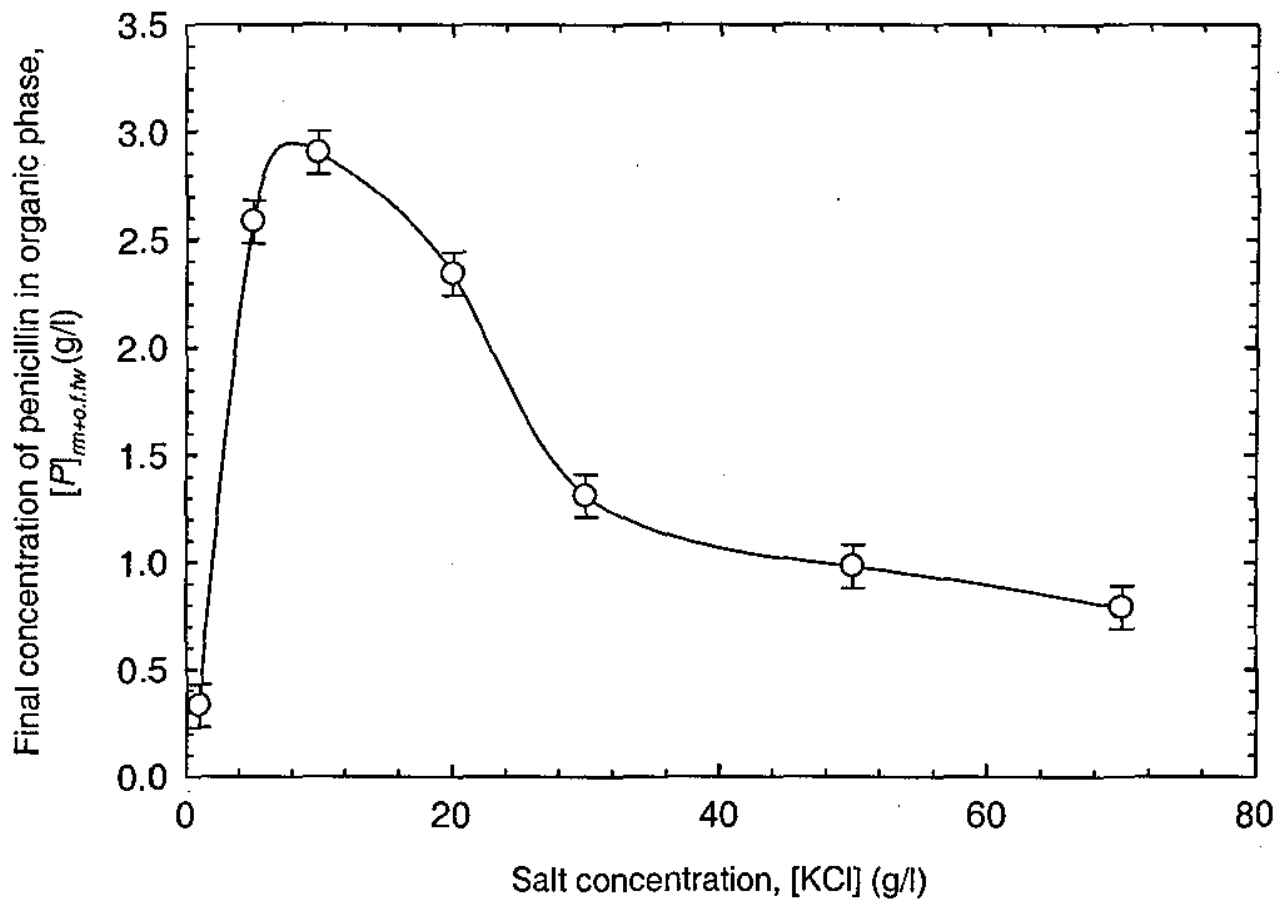

Figure 4.10: Effects of salt concentration on the concentration of penicillin $G$ extracted into the reverse micelle phase. (Temperature, $23 \pm 1^{\circ} \mathrm{C}$; stirring speed, $400 \mathrm{rpm}$ for 10 minutes; initial penicillin $\mathrm{G}$ concentration in the aqueous phase, $3.6 \mathrm{~g} / \mathrm{l} ; \mathrm{pH}, 7.6 \pm 0.5$ ).

The experimental results showed that penicillin $\mathrm{G}$ could be extracted into the reverse micellar phase over a relatively small salt concentration range. With increases salt concentration over $10 \mathrm{~g} / \mathrm{l}$, the extent of antibiotic transfer decreased in spite of the $\mathrm{pH}$ being in the region where electrostatic interaction between the antibiotic and surfactant is attractive. According to Dekker et al. (1989) the ionic strength of the aqueous phase determines the degree of shielding of the electrostatic potential imposed by a charged surfactant head group. They also suggested that by increasing the ionic strength the electrostatic interaction between the charged penicillin molecules and the charged surfactant head groups is reduced. 


\subsubsection{Salt Type}

Salt type also plays an important role in the extraction of a biomolecule from the aqueous to the revese micellar phases (Leodidis and Hatton, 1989; Goto et al., 1999; Cheng and Sabatini, 2001; Kinugasa et al., 2003). To investigate the effects with penicillin $\mathrm{G}$, two salts, $\mathrm{KCl}$ and $\mathrm{CaCl}_{2}$, were dissolved in the aqueous phase and the results are shown in Figure 4.11. It was found that the concentration of penicillin $G$ in the reverse micellar phase is higher when using $\mathrm{CaCl}_{2}$.

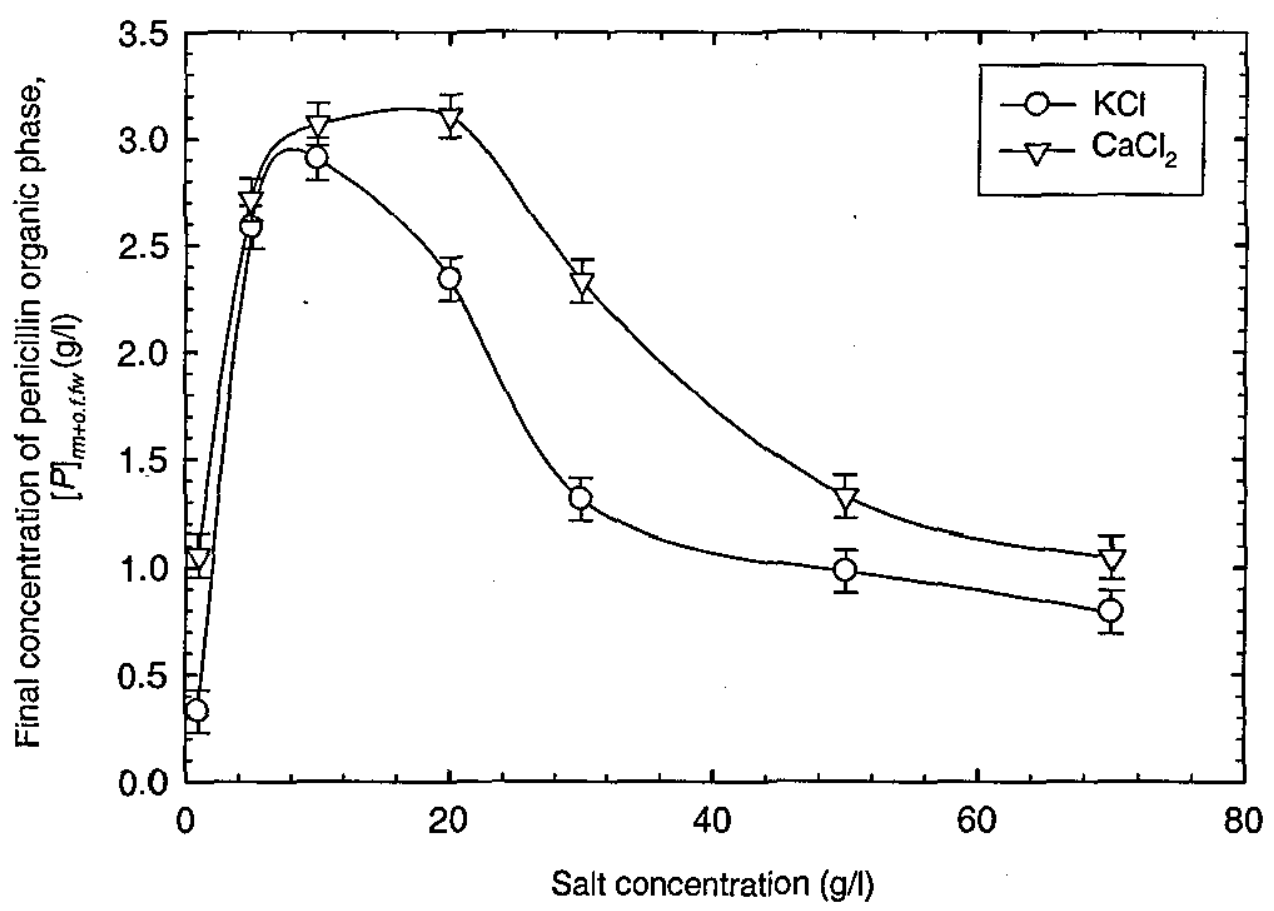

Figure 4.11: Effects of salt type on the concentration of penicillin $\mathrm{G}$ extracted into the reverse micelle phase. (Temperature, $23 \pm 1^{\circ} \mathrm{C}$; stirring speed, $400 \mathrm{rpm}$ for 10 minutes; initial penicillin $\mathrm{G}$ concentration in the aqueous phase, $3.6 \mathrm{~g} / \mathrm{l} ; \mathrm{pH} 7.6 \pm 0.5$ ).

Using a divalent salt like $\mathrm{CaCl}_{2}$ leads to smaller reverse micelle droplets and the ability to solubilise more penicillin is increased. However, it was observed that with $\mathrm{CaCl}_{2}$, the solution became more sensitive to the stirring speed and tended to emulsify more easily. This caused problems with getting a good phase separation, and even when the phases could be separated extra time was needed for the solutions to achieve equilibrium. 


\subsection{Reverse Micelle Formation and Water Content}

The water content in the AOT/isooctane organic phase was measured using a Karl Fischer titration method (as described in Section 3.3.3) after mixing with two $10 \mathrm{~g} / \mathrm{l}$ $\mathrm{KCl}$ solutions, one in its pure state and one containing penicillin $\mathrm{G}$, in order to examine the formation of reverse micelle. Figure 4.12 shows the water content in the organic phase before and after the mixing process. The mixing increased the water content in the reverse micellar phase due to the presence of the AOT (when using the same AOT concentration but without mixing there was no solubilisation of water into the organic phase). The results show that AOT can carry water to the organic phase and form reverse micelle, however, mixing is also very important for inducing solubilisation of water into the organic phase.

After the forward extraction process was complete, the organic phase was clear and transparent indicating that the formation of AOT reverse micelle had been achieved. Such clarity shows that the reverse micelle could solubilise a large amount of water in the organic phase, and since the penicillin molecules can solubilise in water the reverse micelle system would have an ability to extract penicillin molecules into the organic phase. Dokter and co-workers in their study on solubilisation of water in the reverse micelle found that for AOT surfactant in particular, the water molecules in the reverse micelle behave much like the water present in the bulk aqueous phase (Dokter et al., 2006; Dokter et al., 2007). 

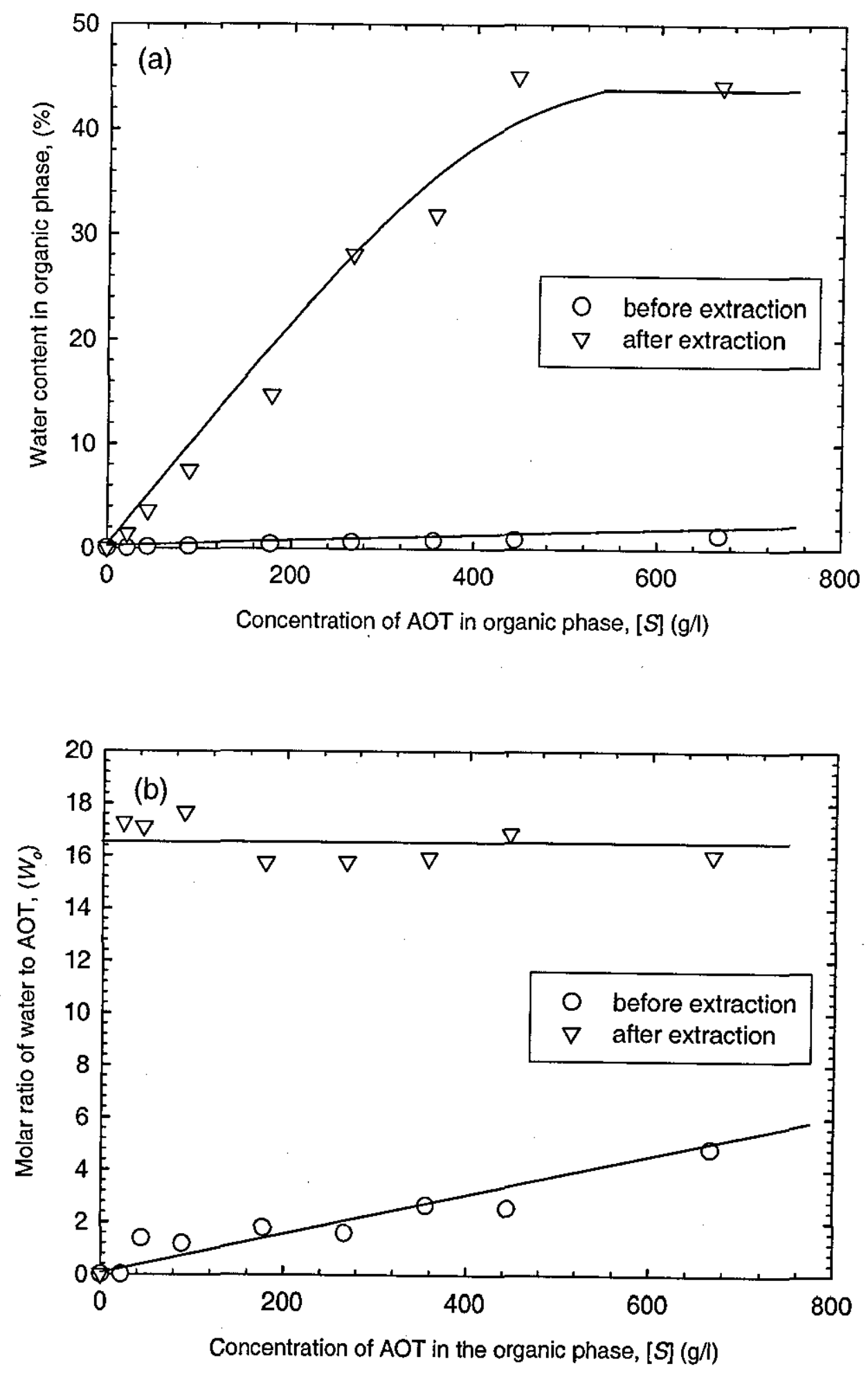

Figure 4.12: Effect of AOT concentration on water uptake into the reverse micelle phase presented as (a) water content, and (b) molar ratio of water to AOT, $W_{o} .([\mathrm{KCl}]=10 \mathrm{~g} / \mathrm{l}$; temperature, $23 \pm 1^{\circ} \mathrm{C}$; stirring speed, $400 \mathrm{rpm}$ for 10 minutes; $\left.\mathrm{pH} 7.6 \pm 0.5\right)$. 
Figure 4.12 shows that water content in the organic phase increases in proportion to the AOT concentration, and without surfactant $([S]=0 \mathrm{~g} / \mathrm{l})$ there is no water uptake. The water content values level off when $[S] \approx 450 \mathrm{~g} / 1$ indicating that the amount of water that can be carried to the reverse micellar phase by the AOT molecules is limited. At $[S]>667 \mathrm{~g} / \mathrm{l}$ the whole solution became a stable emulsion, making it impossible to separate the penicillin molecules from the initial aqueous phase since no phase separation occured after mixing ceased.

The water content of the micellar phase is an important parameter affecting penicillin $\mathrm{G}$ extraction into a reverse micellar system. Previous studies have shown that water content has a strong influence on protein solubilisation and subsequent properties such as the size of reverse micelle (Ono et al., 1996, Kilikian et al., 2000, Naoe et al., 2002). These researchers used the molar ratio of water to surfactant in the micellar organic phase $\left(W_{o}\right)$ in an attempt to understand what affects the characteristics of the reverse micelle, such as their size. The water content analysis is also important in order to determine the structure of reverse micelle and the number of surfactant molecules per reverse micelle (Naoe et al., 2002).

To examine the relationship between water content in the organic phase and penicillin transfer, experiments were carried out to measure the water content in the organic phase in the extraction system both with and without penicillin. The water content in the system without penicillin was found to be higher than that with penicillin. A similar phenomenon was observed by Ono et al. (1996) who used DOLPA reverse micelle to extract haemoglobin. They suggested that water never accompanied the haemoglobin molecules when they were dissolved into the reverse micelle, because haemoglobin extraction consumes the surfactants to form empty reverse micelle. In the penicillin $\mathrm{G}$ case, to fill the reverse micelle in the organic phase the system without penicillin encouraged more reverse micelle containing water compared to the system containing penicillin.

The effect of AOT concentration and the initial mass of penicillin $G$ on the solubilising water was also investigated with experiments carried out at $\mathrm{pH} 7.6$ and $10 \mathrm{~g} / \mathrm{KCl}$ (there was no precipitation or emulsion observed in these experiments). The transparent interface formed, with no insoluble aggregates, indicated that the reverse micelle solubilised successfully in the organic phase. An increase in 
surfactant concentration from $267 \mathrm{~g} / 1$ to $445 \mathrm{~g} / \mathrm{l}$ caused the water uptake to increase from $25 \%$ to $35 \%$. These results agree with studies of protein extraction (Kinugasa et al., 1991; Ichikawa et al., 1992; Frense et al., 1995). For example, Spirovska and Chaudhuri (1998) showed that the percentage of water in the organic phase increased proportionally with the surfactant concentration due to more reverse micelle molecules being solubilised in the organic phase.

To determine a reverse micelle size, other researchers have recommended equations which utilise molar ratio of water to surfactant $\left(W_{o}\right)$ values. From purely geometrical considerations, and assuming the micelles to be monosized with all the surfactant located in a monolayer at the aqueous-organic interface, the reverse micelle radius (water pool radius), $r_{r m}$, is related to $W_{o}$ by Lye (1993);

$$
r_{r m}=\left(\frac{3 V_{\mathrm{H}_{2} \mathrm{O}}}{a_{\mathrm{AOT}}}\right) W_{o}
$$

where $V_{H 2 O}$ is the volume of a water molecule (approximately $0.03 \mathrm{~nm}^{3}$ ) and $a_{A O T}$ the surface area of the surfactant head group $\left(0.5 \mathrm{~nm}^{2}\right)$.

For $[S]>20 \mathrm{~g} / \mathrm{l}$, the value of $W_{o}$ was fairly constant and found to be in the range $15.7 \leq W_{o} \leq 17.6$ and the calculated reverse micelle radii were around $3 \mathrm{~nm}$; the $W_{o}$ values used were taken from the measured experimental data. It has been reported that with an increase of surfactant concentration, the micellar size does not change for most surfactants, which is the case for AOT (Lye, 1993). The results from the current work are in agreement with the results of previous works, which suggest that the calculated value of $r_{r m}$ varies between 1 and $3 \mathrm{~nm}$. For example, Spirovska and Chauduri (1998) revealed that from $W_{o}$ analysis, increasing the surfactant concentration yields an increase in the number of micelles of a constant size. Using the same assumptions that underpin equation (4.4), and further assuming that the projected areas of the AOT head groups are packed at the micelle surface with a porosity of 0.4 , the number of AOT molecules per reverse micelle is calculated to be about 360 . This lies well within the range 100-900 reported by Lye (1993).

Many studies have shown that other parameters such as $\mathrm{pH}$ and salt concentration can affect the solubilisation of biomolecules into the reverse micelle phase. 
However, the results from the current study show that surfactant concentration is the most important parameter affecting the solubilisation of penicillin $\mathrm{G}$ into the reverse micelle phase. Ichikawa et al. (1992), extracting protein with the AOT reverse micelle system, found that even using suitable $\mathrm{pH}$ and salt concentration the extraction will not happen if insufficient AOT concentration is used. They suggested that the solubilising water provides the hydrophilic surroundings that are vital in the reverse micelle extraction process, and that the AOT molecules seemed to take part of the water solubilising agent in the process. Similar to the results from this study, they also found that the maximum water solubilisation increased with increasing surfactant concentration.

\subsection{Thermodynamic Framework for Solubilisation}

A mathematical model could be used to obtain a better qualitative and quantitative understanding of the factors influencing reverse micelle extraction of penicillin $G$. In the reverse micelle extraction experiments, the extraction is influenced by the initial penicillin $\mathrm{G}$ concentration, the salt type and concentration in the aqueous phase, $\mathrm{pH}$, and surfactant concentration. The results show that penicillin is an interfacially active compound that interacts with AOT, with the interfacial association being dependent on both $\mathrm{pH}$ and surfactant concentration. When the concentration ratio $[P]_{\text {aq.fffw }} /[S]$ is high precipitation of the penicillin occurs.

In this work the dependence of the distribution coefficient on penicillin concentration, AOT concentration, and $\mathrm{pH}$ has been measured. When the distribution coefficient of forward extraction, $K_{E . f w}$, is plotted against the concentration of penicillin in the aqueous phase, $[P]_{\text {aq.f.fw }}$, (all other parameters being held constant) and the experimental data extrapolated back to $[P]_{a q . f . f w}=0$, a value for the distribution coefficient at infinite dilution for forward extraction $\left(K_{E . f w}^{\infty}\right)$ is found (see Figure A2-1 as shown in Appendix 2). Following this procedure, the results for $\mathrm{pH} 1.65$ and 7.6 are shown in Figure 4.13. 


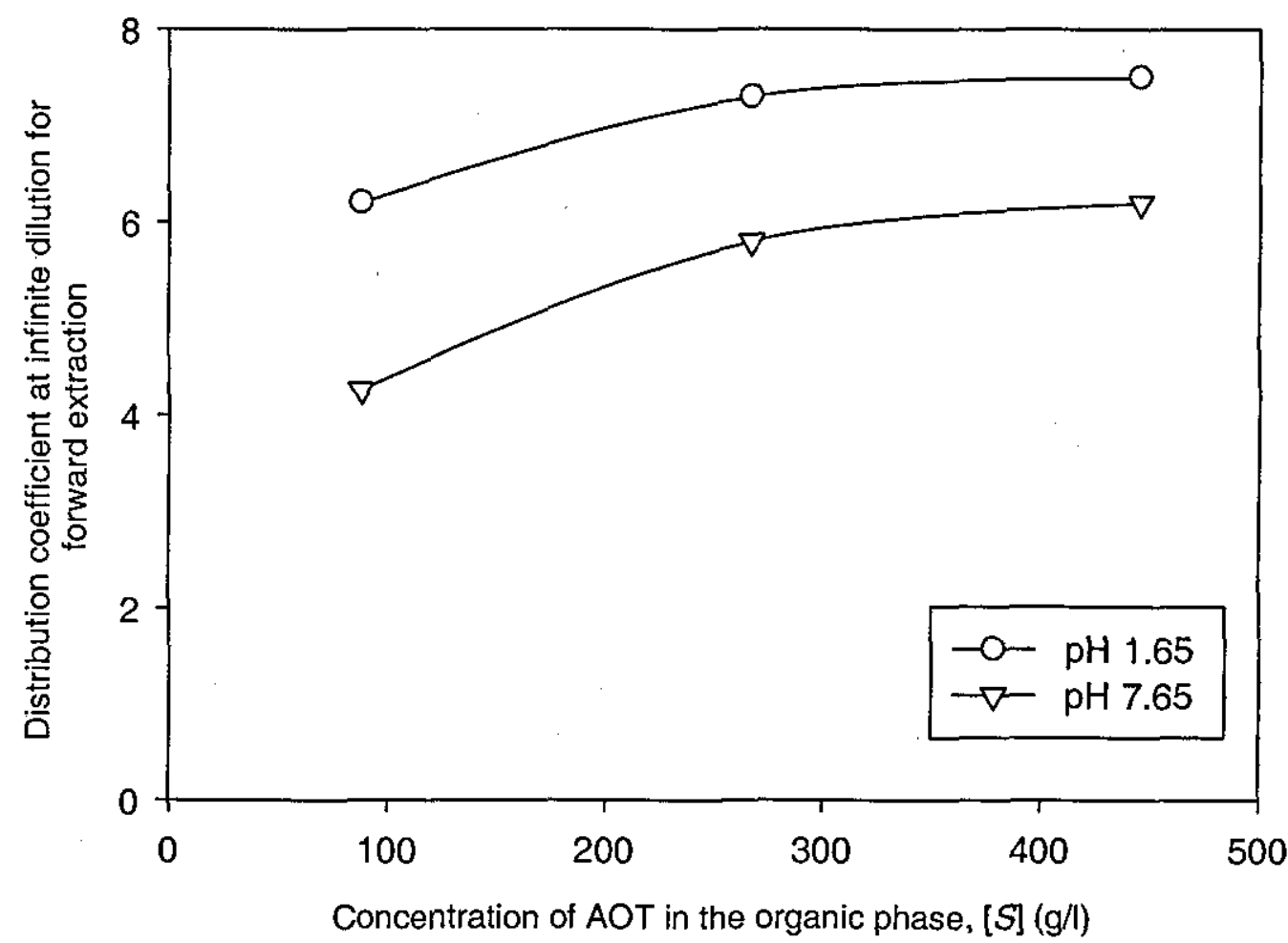

Figure 4.13: Variation of distribution coefficient at infinite dilution for forward extraction $\left(K_{E, f w}^{\infty}\right.$ ) with AOT concentration and $\mathrm{pH}$. (Temperature, $23 \pm 1^{\circ} \mathrm{C}$; stirring speed, $400 \mathrm{rpm}$ for 10 minutes; initial penicillin $\mathrm{G}$ concentration in the aqueous phase, $3.6 \mathrm{~g} / \mathrm{l})$.

The distribution coefficient at infinite dilution for forward extraction depends markedly on $\mathrm{pH}$ and surfactant concentration. At both $\mathrm{pH}$ values, $K_{E . f w}^{\infty}$ increases as the surfactant concentration increases, with higher values being associated with $\mathrm{pH}$ 1.65. However, one should notice that the limitation of using a pH as low as 1.65 is that the extraction becomes troublesome due to formation of a precipitate except at very low penicillin concentrations. So whilst there is clearly a $\mathrm{pH}$ effect on $K_{E . f w}^{\infty}$, in protein, other factors have to be taken into account.

\subsubsection{Distribution Coefficient at Infinite Dilution}

Modelling of the solubilisation of penicillin $G$ in the reverse micelle was carried out for the forward extraction. A Langmuir model and an adsorption model, which is refered to as the 'Brandani model' (Brandani et al., 1994) were used to find the 
dependency of the system to process parameters. The experimental results are reported in Figure 4.14, and Tables A4-1 andA4-2 as shown in Appendix 4.

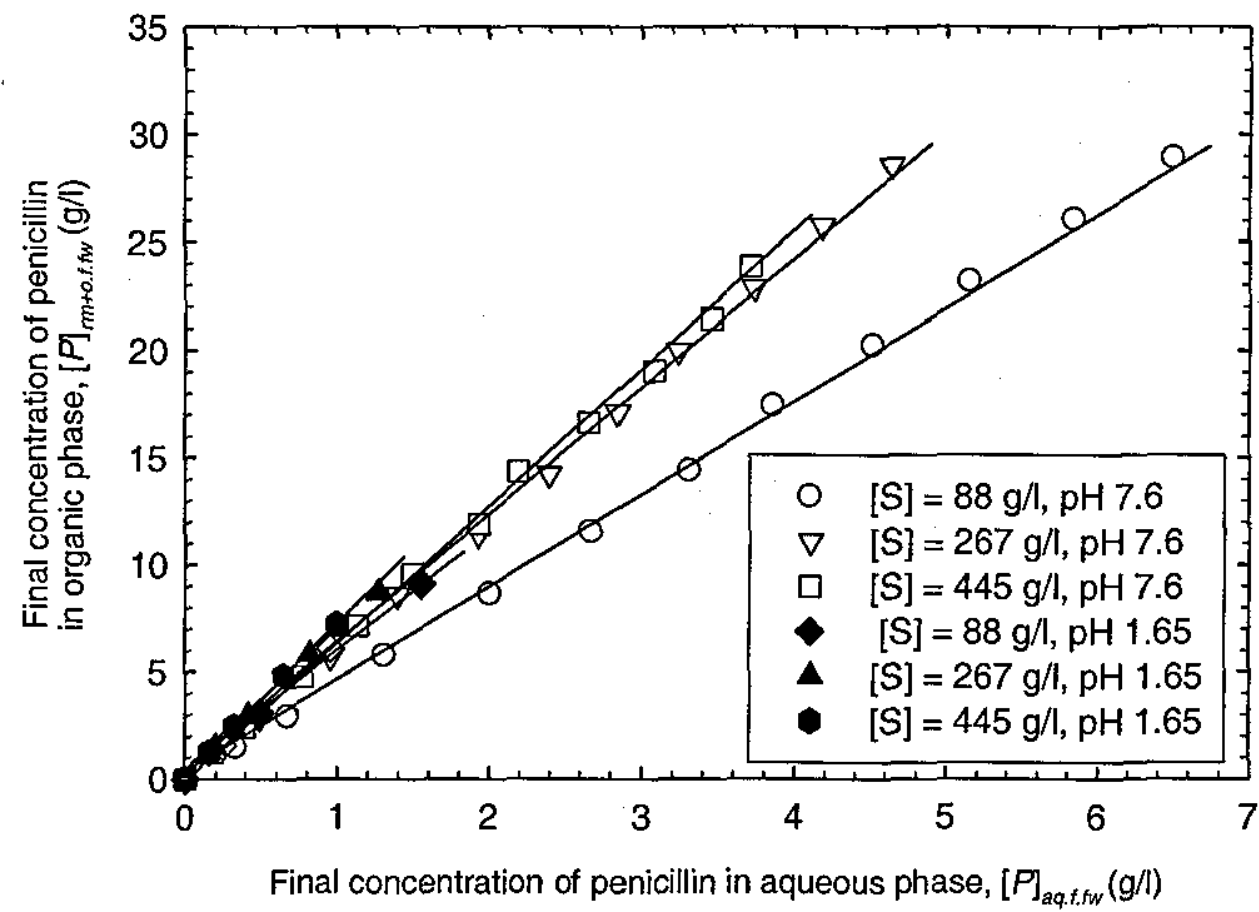

Figure 4.14: Isotherms of penicillin $\mathrm{G}$ at $\mathrm{pH} 7.6$ and 1.65. ([KCl] $=10 \mathrm{~g} / 1$; temperature, $23 \pm 1^{\circ} \mathrm{C}$; stirring speed, $400 \mathrm{rpm}$ for 10 minutes).

The final concentration of penicillin $G$ in the aqueous phase for forward extraction $\left([P]_{\text {aq.f.fw }}\right)$ and the final concentration of penicillin $\mathrm{G}$ in the reverse micelle organic phase $\left([P]_{r m+o . f f w}\right)$ were measured at surfactant concentrations of 88,267 and $445 \mathrm{~g} / \mathrm{l}$. For each surfactant concentration two different $\mathrm{pH}$ values were considered, namely 7.6 and 1.65. The models described in Sections 4.7.1.1 and 4.7.1.2 fitted to each set of experimental data.

\subsubsection{Data Fitted with the Langmuir Model}

The basic idea behind the Langmuir model is coverage of the surface by a monomolecular layer. The Langmuir model for solubilisation of penicillin $G$ during forward extraction is given by: 


$$
[P]_{r m+o . f . f w}=\frac{a_{L . f w}[P]_{a q . f . f w}}{1+b_{L . f w}[P]_{a q . f . f w}}
$$

where $a_{L . f w}$ and $b_{L . f w}$ are the adjustable parameters. The distribution coefficient for the Langmuir model can be written as:

$$
K_{L . f w}=\frac{[P]_{r m+o . f . f w}}{[P]_{a q . f . f w}}=\frac{a_{L . f w}}{1+b_{L . f w}[P]_{a q . f . f w}}
$$

At infinite dilution $[P]_{\text {aq.f.fw }} \rightarrow 0$ and

$$
K_{L . f w}^{\infty}=a_{L . f w}
$$

where $K_{L . f w}^{\infty}$ is the distribution coefficient from the Langmuir model at infinite dilution.

Fitting of the Langmuir model to the experimental data was done using equation (4.6) and the SigmaPlot curve fitting programme. Figures 4.15 shows comparison between experimental data and the fitted models at $\mathrm{pH} 7.6$ and $[S]=88 \mathrm{~g} / 1$ (see Figures A4.2-1 - A4.2-5 as shown in Appendix 4 for other fitted plots at different $\mathrm{pH}$ and $[S])$. The results show that the model has a good agreement with the experimental data. From the fitted results, $K_{L . f w}^{\infty}$ (for the Langmuir model $K_{L . f w}^{\infty}=a_{L . f w}$ ) and $b_{L . f w}$ values were obtained for both pH's as shown in Figures 4.16 and Table 4.1 respectively. Figure 4.16 shows that increasing $[S]$ causes $K_{L . f w}^{\infty}$ to increase. There is a weak relationship between $K_{L . f w}^{\infty},[S]$ and $\mathrm{pH}$ but the trends for both $\mathrm{pH}$ values are similar. Figure 4.16 also shows that the $b_{L . f w}$ dependent on $\mathrm{pH}$ at low $\mathrm{pH}$, and at higher $\mathrm{pH}$ the value of $b_{L f w}$ is almost independent of [S]. 


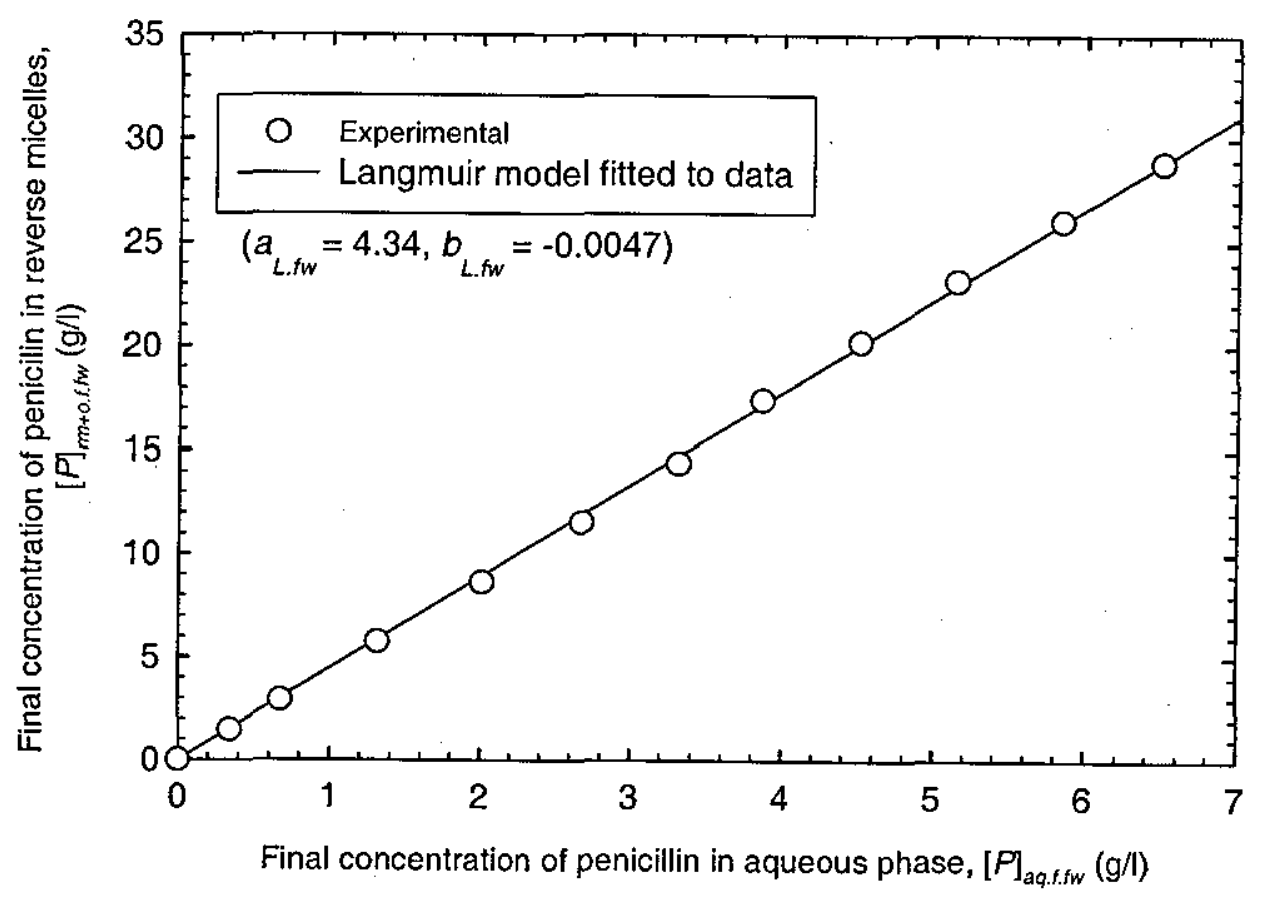

Figure 4.15: Langmuir model fitted to experimental data at $\mathrm{pH} 7.6$ and $[S]=88 \mathrm{~g} / \mathrm{l}$.

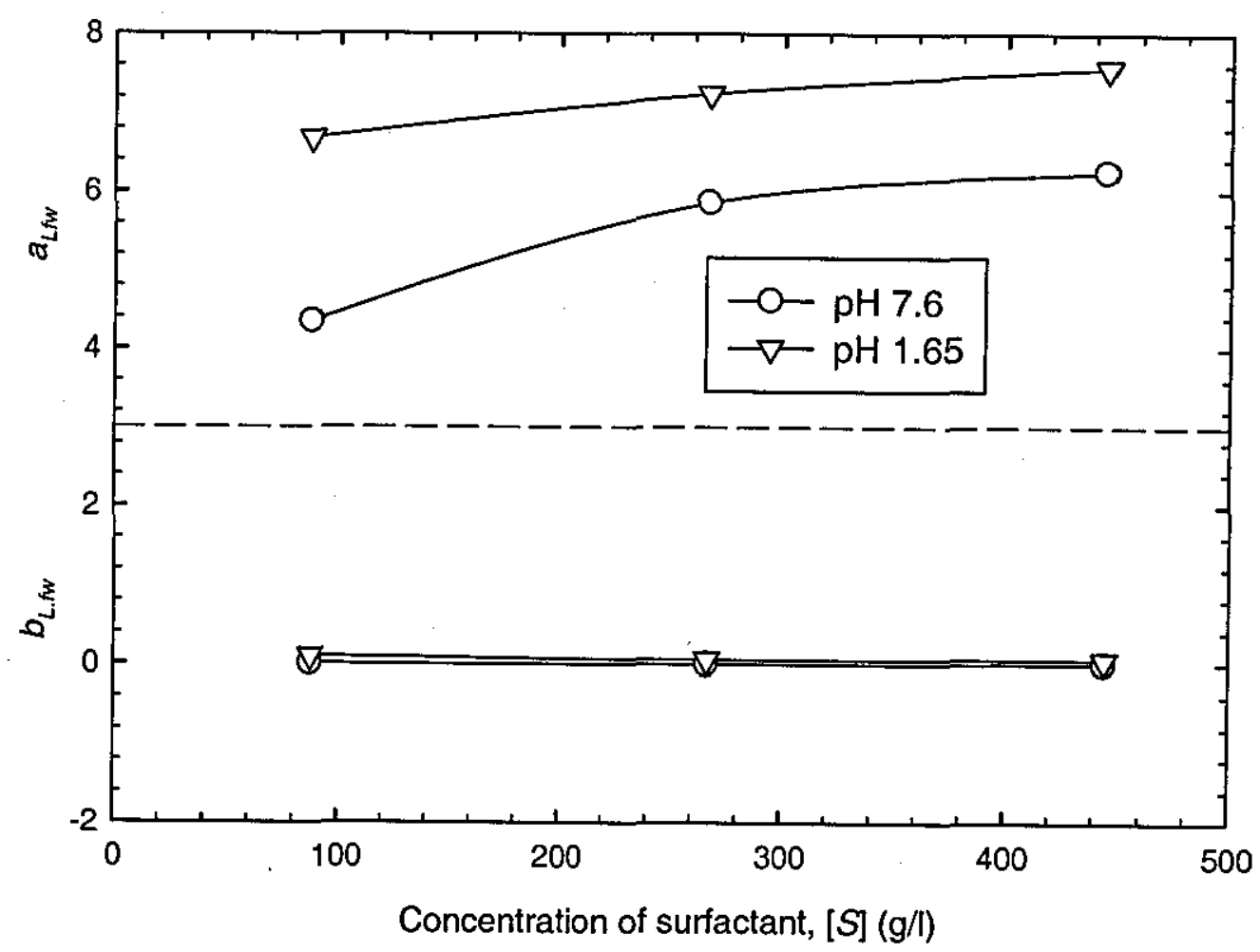

Figure 4.16: Partition coeffiecient at infinite dilution of Langmuir model for forward extraction, $a_{L . f w}\left(=K_{L . f w}^{\infty}\right)$ and $b_{L f w}$ values at different $\mathrm{pH}$ and surfactant concentration. 
Table 4.1: $a_{L . f w}\left(=K_{L . f w}^{\infty}\right)$ and $b_{L . f w}$ values at different $\mathrm{pH}$ and surfactant concentration from the Langmuir model for forward extraction.

\begin{tabular}{|c|c|c|c|}
\hline \multicolumn{2}{|c|}{ Experimental parameters } & \multirow{2}{*}{$a_{L . f w}=K_{L . f w}^{\infty}$} & $b_{L . f w}$ \\
\hline$[S]$ & $\mathrm{pH}$ & & -0.0047 \\
\hline 88 & 7.6 & 4.34 & -0.0112 \\
267 & 7.6 & 5.86 & -0.0024 \\
445 & 7.6 & 6.25 & 0.0889 \\
88 & 1.65 & 6.67 & 0.0492 \\
267 & 1.65 & 7.24 & 0.0532 \\
\hline 445 & 1.65 & 7.57 & \\
\hline
\end{tabular}

Thus, the Langmuir model shows a good agreement when fitted to the experimental data at both values of $\mathrm{pH}$. However, the concept of Langmuir model is a surface adsorption model. Since this model only represents the absorption phenomena that occurr at the interface, it does not correctly represent the thermodynamics of the physical system. The absorption process in the reverse micelle water pool is important since the hydrophilic molecules tend to exist in the water pools (Adachi $e t$ al., 1991; Yonker et al., 2003). Moreover, application of equation (3.12) in Section 3.6.2 proved that in this work penicillin $\mathrm{G}$ does solubilise in the water pools.

\subsubsection{Data Fitted with the Brandani Model}

Brandani et al. (1994) developed an adsorption model that considered an aqueous phase in equilibrium with an organic phase with many identical sites made up of reverse micelle onto each of which one molecule of the adsorbing material (solute) can be entrapped. The concentration of solute in the reverse micelle was then expressed as:

$$
[P]_{r m+o . f . f w}=\frac{a_{B . f w} b_{B . f w}[S][P]_{a q . f . f w}}{1+b_{B . f w}[P]_{a q . f . f w}}
$$

where $a_{B . f w}$ and $b_{B . f w}$ are adjustable parameters; $b_{B . f w}$ is thought to be a parameter characteristic of the inner surface of reverse micelle, the solute, and the temperature, but is independent of $[P]_{\text {aq.ffw. }}[S]$ is the concentration of surfactant. Using this model, the distribution coefficient from the Brandani model $\left(K_{B . f w}\right)$ depends on both $[S]$ and $[P]_{\text {aq.f.fw }}$ and can be written as: 


$$
K_{B . f w}=\frac{[P]_{r m+o . f . f w}}{[P]_{a g . f . f w}}=\frac{a_{B . f w} b_{B . f w}[S]}{1+b_{B . f w}[P]_{a q . f . f w}}
$$

At infinite dilution $[P]_{\text {aq.f.fw }} \rightarrow 0$ and

$$
K_{B . f w}^{\infty}=a_{B, f w} b_{B . f w}[S]
$$

or

$$
\ln K_{B . f w}^{\infty}=\ln a_{B . f w}+\ln b_{B . f w}+\ln [S]
$$

where $K_{B . f w}^{\infty}$ is the distribution coefficient of the Brandani model at infinite dilution.

A slightly different equation based on the mass-action principle was derived by Woll and Hatton (1989) which indicated a pH dependency:

$$
\ln K^{\infty}=\ln a+\ln b+(c+d \cdot p H) \ln [S]
$$

The Woll and Hatton model assumed a pseudo-chemical equilibrium for complexation of protein molecules with the hydrophobic tails of reverse micelle as empty sites. Also using the mass-action principle, Rabie and Vera (1998) developed an ion exchange model for protein partitioning, based on the assumption that at equilibrium the concentration of the protein in both the aqueous and reverse micellar phases are the same and that the activities of components in a solution are equal to their concentration.

The fitting of the Brandani model to experimental data was done using equations (4.9). $a_{B . f w}$ and $b_{B . f w}$ are the parameters from the fitting. Figures 4.17 shows a good agreement between experimental data and the Brandani model at $\mathrm{pH} 7.6$ and $[S]=88$ $\mathrm{g} / \mathrm{l}$ (other plots for different $\mathrm{pH}$ and $[S]$ were shown in Figures A4.3-1 - A4.3-5 in Appendix 4). The results show that the experimental data exhibit behaviour of a Langmurian type, modified by the conditions of the Brandani model. The results of data fitting from this work show similarity to work of Brandani et al. (1994) even though a very different solute has been used. They asserted that the distribution 
coefficient depends also on the biomolecule (protein in their case) concentration in the aqueous phase.

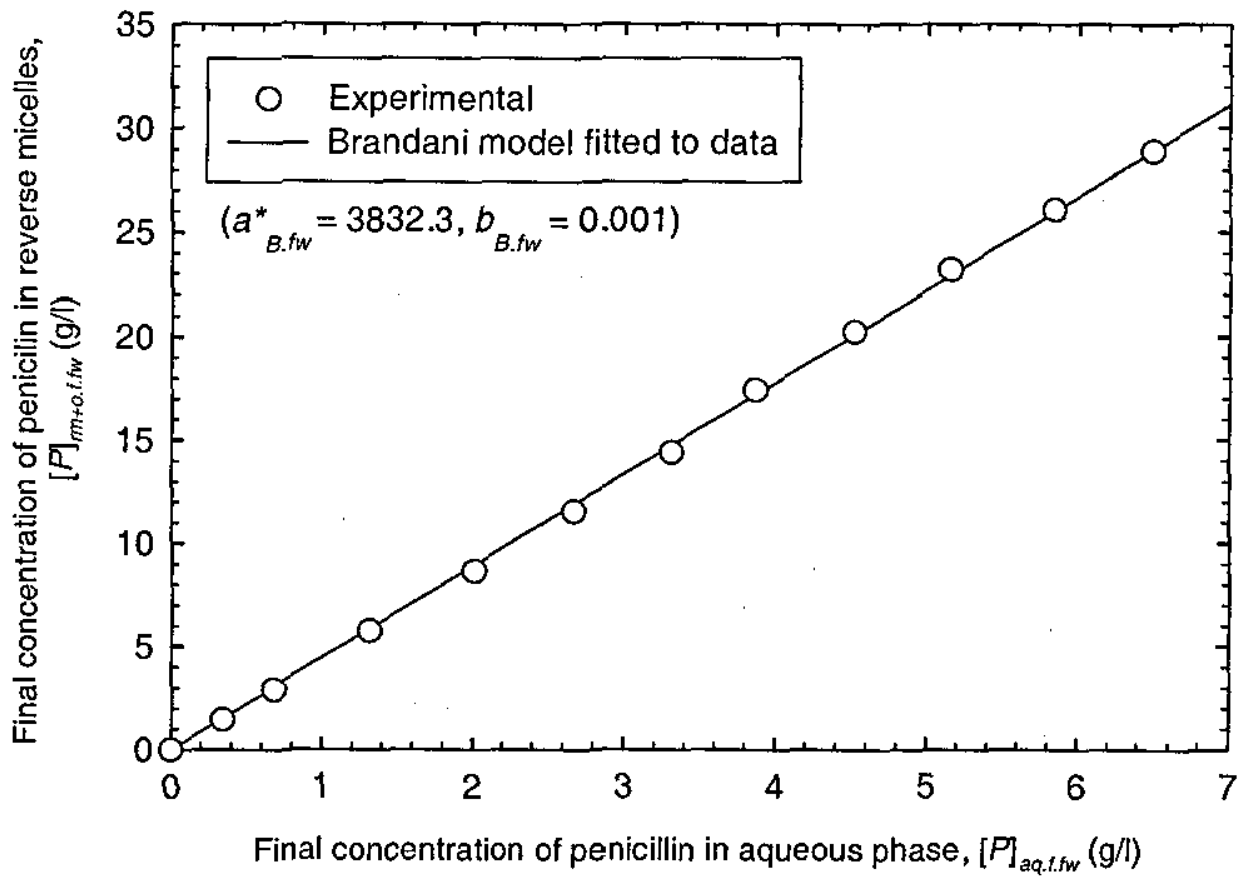

Figure 4.17: Brandani model fitted to experimental data at $\mathrm{pH} 7.6$ and $[S]=88 \mathrm{~g} / \mathrm{l}$.

$$
\left(\mathrm{a}_{B \cdot f w}=a_{B . f w}[S]\right) \text {. }
$$

The distribution coefficient at infinite dilution from the Brandani model, $K_{B . f w}^{\infty}$, can be calculated from the $a_{B . f w}$ and $b_{B . f w}$ values as shown in equation (4.11), and equation (4.13) suggests that $a_{B . f w}$ and/or $b_{B . f w}$ values may be dependent on $\mathrm{pH}$ and/or [S]. Figure 4.18 shows that $b_{B . f w}$ has not change with $\mathrm{pH}$ at any [S]. Figure 4.18 also shows that the behaviour of the distribution coefficient at infinite dilution from the Brandani model for forward extraction, $K_{B . f w}^{\infty}$, calculated from equation (4.11) and plotted as a function of $\mathrm{pH}$ and surfactant concentration. The results show a similar trend for both $\mathrm{pH}$ values, that is, $K_{B . f w}^{\infty}$ increases when increasing [S]. The Brandani model is found to more relevant to this work as it describes the thermodynamic distribution of solutes in both the interfacial phase and the internal region of the reverse micelle. The model shows an approximation to a real physical system. In this work penicillin $\mathrm{G}$ is considered to stay in the water phase and the mass of penicillin at the surface is small compared to the mass in the water pool. The trend of the results obtained from the current work is similar to the work done by Brandani 
et al. (1994) and Woll and Hatton (1989) which indicates that there is a similar behaviour in the solubilisation process even when two different systems are used (the other works used protein as the biomolecule). In this work it is found that the solubilisation of penicillin $\mathrm{G}$ also depends on $\mathrm{pH}$, which is a similar result to Woll and Hatton (1989) as shown by equation (4.12).

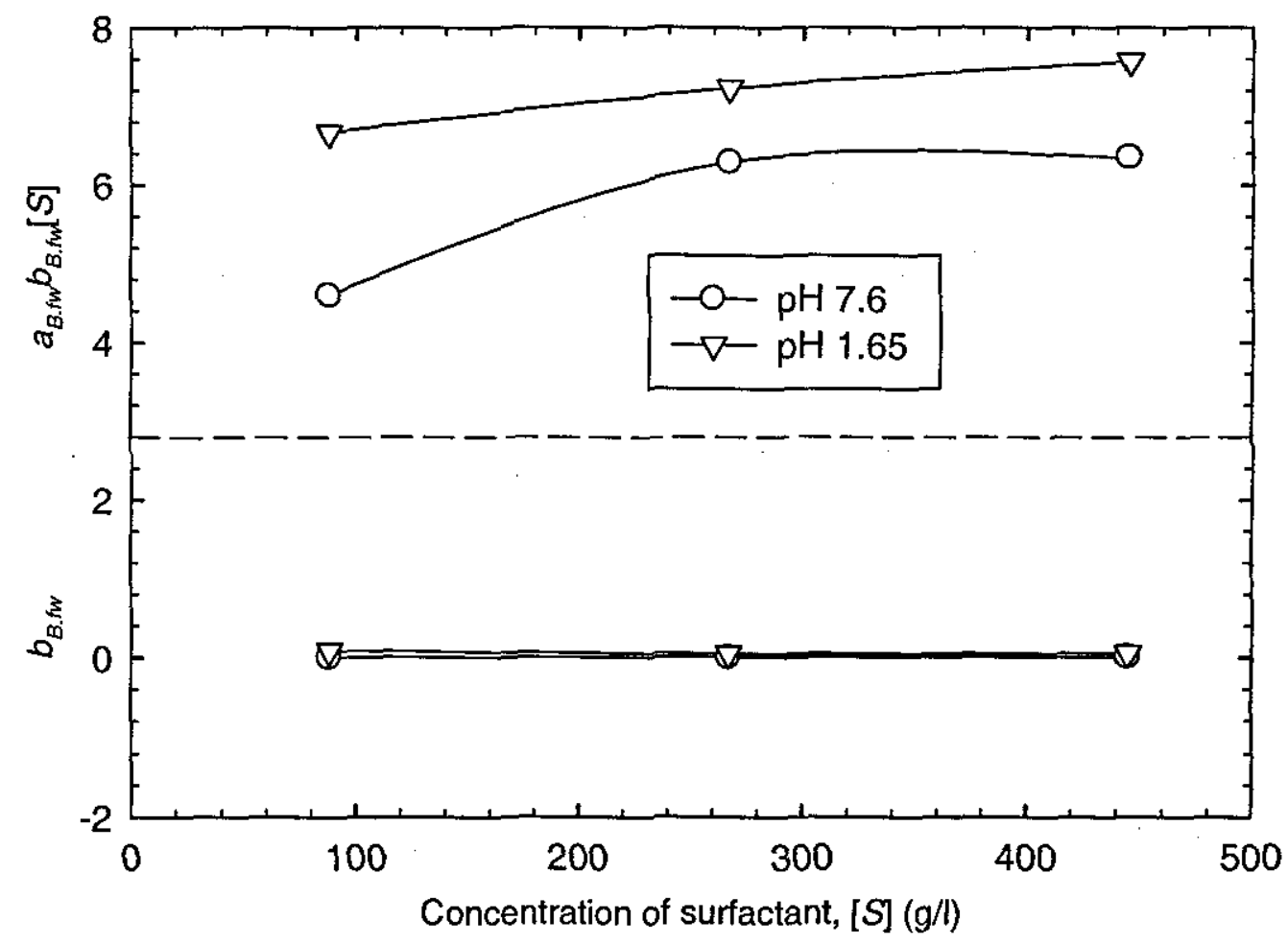

Figure 4.18: Partition coeffiecient at infinite dilution of Brandani model for forward extraction, $a_{B . f w} b_{f w}[S]\left(=K_{B . f w}^{\infty}\right)$ and $b_{\mathrm{B} . f w}$ values at different $\mathrm{pH}$ and surfactant concentration.

The values of the distribution coefficients at infinite dilution, $K^{\infty}$, from the experimental data $\left(K_{E . f w}^{\infty}\right)$, the Langmuir model $\left(K_{L . f w}^{\infty}\right)$, and the Brandani model $\left(K_{B . f w}^{\infty}\right)$ are summarised in Table 4.2. Both models show good agreement with the experimental data. 
Table 4.2: Comparison of distribution coefficient, $K^{\infty}$, between the experimental results, the Langmuir model and the Brandani model.

\begin{tabular}{|c|c|c|c|c|}
\hline \multicolumn{2}{|c|}{ Experimental parameters } & $K_{E . f w}^{\infty}$ & $K_{L . f w}^{\infty}$ & $K_{B . f w}^{\infty}$ \\
\hline$[\mathrm{S}]$ & $\mathrm{pH}$ & 4.27 & 4.34 & 4.60 \\
\hline 88 & 7.6 & 5.35 & 5.86 & 6.29 \\
267 & 7.6 & 6.23 & 6.25 & 6.35 \\
445 & 7.6 & 6.21 & 6.67 & 6.67 \\
88 & 1.65 & 7.25 & 7.24 & 7.23 \\
267 & 1.65 & 7.50 & 7.57 & 7.57 \\
445 & 1.65 & & & \\
\hline
\end{tabular}

\subsection{Conclusions}

Experimental results for the solubilisation of penicillin $G$ into reverse micelle of AOT (forward extraction) have been presented and fitted with two models from the literature. The results show that the concentration of penicillin $G$ in the organic phase depends primarily on the $\mathrm{pH}$ and surfactant concentration, with the salt type and concentration in the aqueous phase also being important. However, the effect of salt type and concentration is sufficient to signify that extraction from a broth needs to be assessed experimentally.

The results show that penicillin $\mathrm{G}$ is an interfacially active compound that interacts with AOT surfactant, with the interfacial association being dependent on both $\mathrm{pH}$ and surfactant concentration. When the concentration ratio $[P]_{\text {aq. } f}[[S]$ is high, precipitation of the penicillin occurs, which limits the possible use of reverse micellar extraction at low values of $[P]_{a q . f w}$.

The distribution coefficient favours solubilisation of penicillin into the reverse micelle at moderate AOT concentrations. There is no saturation limit of penicillin $\mathrm{G}$ transferred into the reverse micelle; however there is a limit of surfactant concentration beyond which the concentration of penicillin $G$ transferred into reverse micelle remains the same. The distribution coefficient at infinite dilution for forward extraction $\left(K_{f w}^{\infty}\right)$ has been shown to be a function of both $\mathrm{pH}$ and surfactant concentration. Similar trends in the value of $K_{f w}^{\infty}$ were observed at different $\mathrm{pH}$ 
values; $K_{f w}^{\infty}$ increases as the surfactant concentration is increased. However, although $K_{f w}^{\infty}$ is higher at $\mathrm{pH} 1.65$ the extraction is difficult due to precipitation when high initial penicillin concentration is used.

Reverse micelle formation affects the volume of water transferred into the organic phase (this water is held within the reverse micelle). From measurements of the water transferred, the size of the revere micelles was estimated to be about $3 \mathrm{~nm}$ and the number of AOT molecules per reverse micelle about 360 . These values are in broad agreement with previous work using AOT to extract proteins.

The Langmuir and Brandani models were adopted to compare with the experimental data. Equations from both models showed that surfactant concentration plays an important role insolubilisation. Whilst some of the thermodynamic models for solubilisation suggest that there should be an effect of $\mathrm{pH}$ (for example, equation (4.13)), others do not (see equation (4.12)). $K_{f w}^{\infty}$ from the experimental data $\left(K_{E . f_{w}}^{\infty}\right)$, the Langmuir model $\left(K_{L . f w}^{\infty}\right)$ and the Brandani model $\left(K_{B . f w}^{\infty}\right)$ were compared and both models showed a good agreement with the experimental data.

Since the Langmuir model only represents the absorption phenomena that occur at the interface, the Brandani model is more relevant to this work since it describes the thermodynamic distribution for both the interface and the reverse micelle phase. The Brandani model is therefore a better approximation to a real physical system. 


\section{CHAPTER FIVE}

\section{Results and Discussion - Backward Extraction}

\subsection{Introduction}

Chapter 5 presents results from investigations of the factors which encourage penicillin $G$ to solubilise from reverse micelle into a fresh aqueous phase (backward extraction). Discussions focus on the additional volume of the aqueous phase that occurred after backward extraction, the effect of salt concentration and $\mathrm{pH}$, the effect of the initial mass of penicillin $\mathrm{G}$ before forward extraction $\left(P_{\text {aq.i.fw }}\right)$ and surfactant concentration. In a similar manner to Chapter 4 , this chapter also interprets the thermodynamic framework for solubilisation by using Langmuir and Brandani models. The additional information related to the results for backward extraction can be found in Appendix 3.

In a reverse micelle process, it is desirable to release solutes from the reverse micelle so that a quantitative recovery of purified bioproduct can be achieved (Carlson and Nagarajan, 1992; Mathew and Juang, 2007). Despite the importance of backward extraction, many researchers working on protein extraction found that the protein is more difficult to solubilise from reverse micelle back into an aqueous phase than vise versa (Dungan et al., 1991; Pires et al., 1996; Zhang et al., 1999). In the current work similar problem occured during backward extraction, since large mass of penicillin $G$ that were present in the reverse micelle phase after forward extraction $\left(P_{r m+o . f f w}\right)$ stayed in the reverse micelle and did not transfer into a fresh aqueous phase. These results originate from the strong interaction between the penicillin $G$ molecule and the composition of reverse micelle (Dekker et al., 1990; Pires et al., 1996; Lee et al., 2001); the difficulties of breaking this strong interaction are discussed toward the end of the chapter. In the Chapter 4 , the forward extraction process showed that the mass of penicillin $G$ transferred from the aqueous phase to the reverse micelle phase is very high and therefore encouraging, since almost the 
entire initial mass was solubilised into the reverse micelle. Penicillin G molecules are easier to solubilise into the reverse micelle during forward extraction, but difficult to transfer back to a new aqueous phase even by manipulating operational parameters.

\subsection{Factors Affecting Backward Extraction}

Backward extraction tends to be difficult compared to forward extraction; Carlson and Nagarajan (1992) concluded that different biomolecules respond differently to the variation in backward solution properties such as $\mathrm{pH}$ and salt concentration. Mathew and Juang (2007) suggested that the problems that occur during backward extraction are due to a strong interaction between the solubilised biomolecule and the reverse micelle. For penicillin $G$ recovery by backward extraction, we need to investigate if reverse micelle-penicillin $G$ interaction is strong enough to prevent the penicillin $\mathrm{G}$ solubilising from the reverse micelle phase into the back aqueous phase, even with hydrophilic nature of penicillin $\mathrm{G}$.

A conclusion drawn from the forward extraction of penicillin $G$ was that solubilisation into the reverse micelle is mainly driven by the electrostatic interaction between the positive charge of penicillin $\mathrm{G}$ and the opposite charge of AOT. This notion was taken into consideration when carrying out backward extraction of penicillin G. Hence, in order to decrease the electrostatic interactions between penicillin $\mathrm{G}$ and $\mathrm{AOT}$, the $\mathrm{pH}$ and salt concentration of the fresh aqueous phase were increased. By controlling these two parameters it was possible to promote backward extraction of penicillin $G$ into the aqueous phase. A schematic diagram of the backward extraction process was shown in Section 3.5 (Figure 3.8).

Figure 5.1 shows the mass of penicillin $\mathrm{G}$ in the backward aqueous phase $\left(P_{\text {aqf.bw }}\right)$ at different backward extraction times (mixing times). It is important to obtain information on how long the back extraction process will take in order to produce equilibrium solubilisation for penicillin $\mathrm{G}$ transfer. The results show that the mixing time must be carried out for more than 5 minutes in order to achieve equilibrium, as the contact time was prolonged so the penicillin $\mathrm{G}$ recovery reached a plateau. 


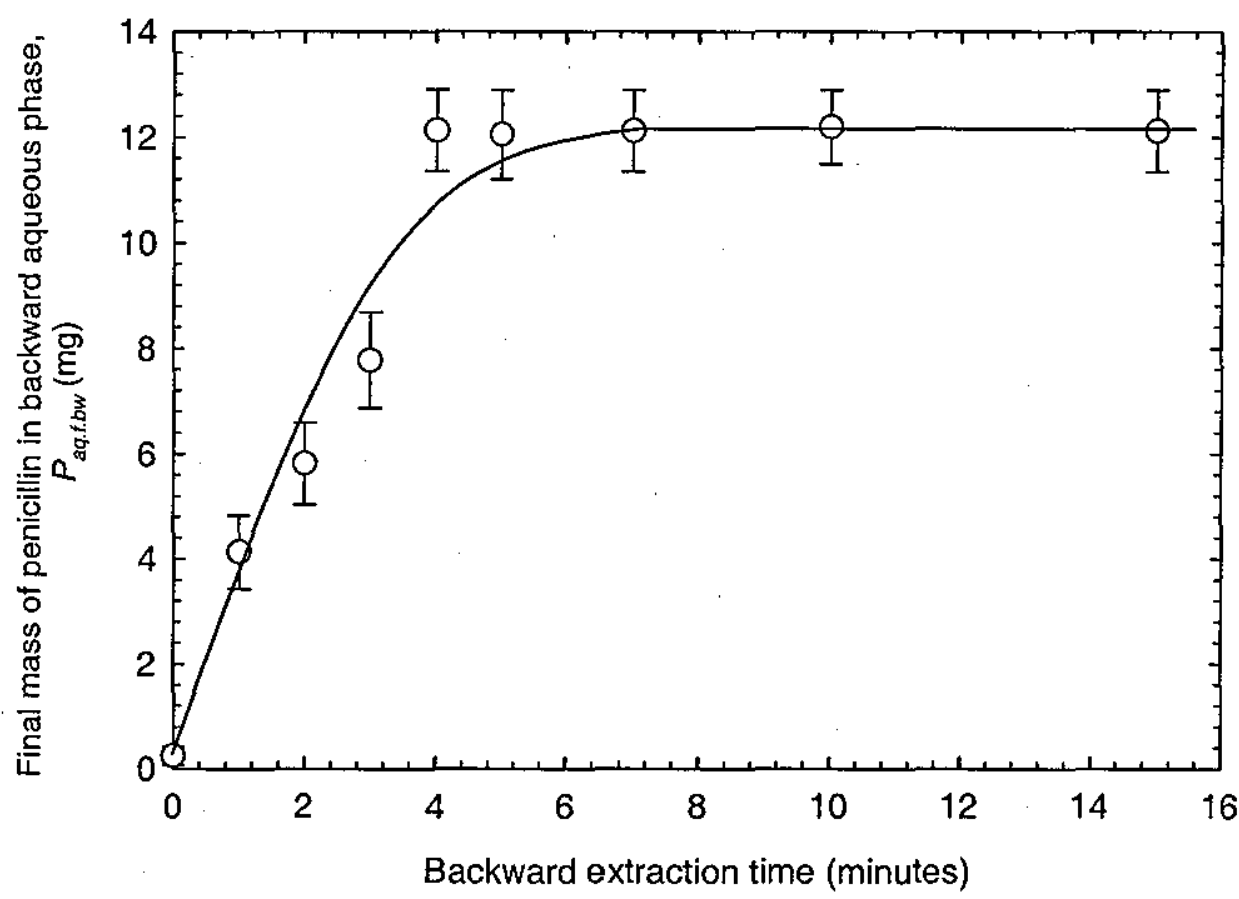

Figure 5.1: The effect of backward extraction time on the mass of penicillin $\mathrm{G}$ in the backward aqueous phase. $\left([\mathrm{KCl}]=37 \mathrm{~g} / \mathrm{l}\right.$; temperature, $23 \pm 1^{\circ} \mathrm{C}$; stirring speed, $400 \mathrm{rpm} ; \mathrm{pH}$ 7.6 \pm 0.5 ; initial mass of penicillin $\mathrm{G}$ before backward extraction, $29 \mathrm{mg} ;[S]=88 \mathrm{~g} / \mathrm{l}$ ).

Many researchers have discovered that the precipitation tends to occur at the interface (Nishiki et al., 1995; Krieger et al., 1997; Shin et al., 2003), or in the aqueous phase (Naoe et al., 1999) during backward extraction. In the current work the phases were easily separated without any precipitation. However, when high stirring speed was used, especially for high AOT concentrations, the phases could not be separated due to the probable formation of a stable emulsion.

During a backward extraction experiment, the stirring speed was controlled to 400 rpm. The effect of the settling period on the concentration of penicillin $G$ in the organic phase at different AOT concentrations was studied and the results are shown in Figure 5.2. The settling period for backward extraction was much shorter than in forward extraction. In forward extraction, up to 24 hours settling time was required to achieve equilibrium solubilisation of penicillin $\mathrm{G}$ into the reverse micelle. Based on the observations during experimental work, there was no significant difference 
between 10 hours and 25 hours settling times, indicating that the penicillin solubilisation had reached the equilibrium state at 10 hours.

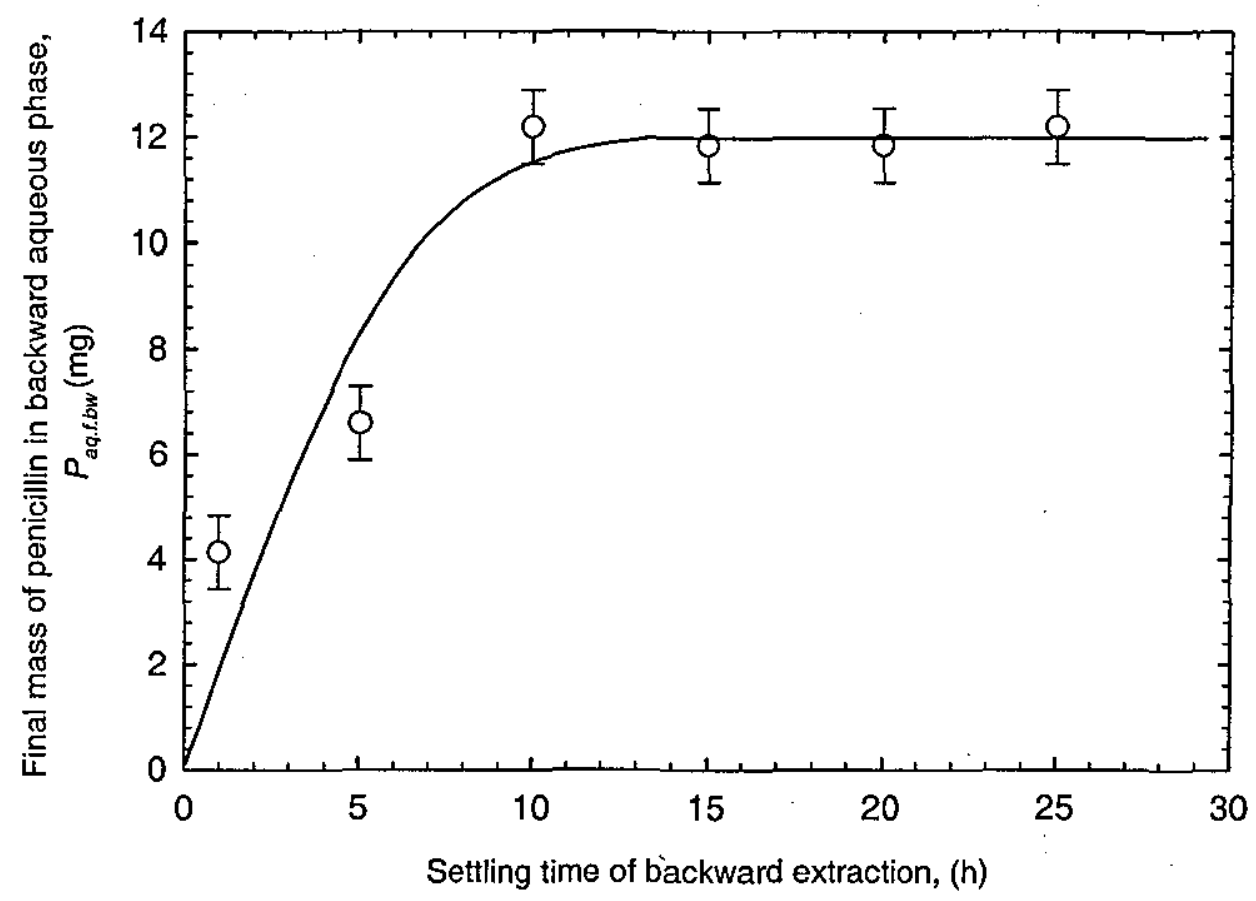

Figure 5.2: Effect of settling time on the mass of penicillin $G$ in the aqueous phase for backward extraction process. $\left([\mathrm{KCl}]=37 \mathrm{~g} / \mathrm{l}\right.$; temperature, $23 \pm 1^{\circ} \mathrm{C}$; stirring speed, $400 \mathrm{rpm}$ for 5 minutes; $\mathrm{pH} 7.6 \pm 0.5$; initial mass of penicillin $\mathrm{G}$ before backward extraction, $29 \mathrm{mg}$;

$$
[S] \simeq 88 \mathrm{~g} / \mathrm{l}) \text {. }
$$

\subsubsection{Additional Volume of the Backward Aqueous Phase}

At different AOT concentrations, there was an increase in the final volume of the backward aqueous phase $\left(V_{a q . f . b w}\right)$ that was proportional to a decrease in the final volume of the backward reverse micelle phase $\left(V_{r m+o . f . b w}\right)$; it should be noted that 'AOT concentrations' refers to the surfactant concentration used in the forward extraction. The results from forward extraction showed that increasing AOT concentration increases the concentration of penicillin $G$ in the reverse micelle. In order to investigate the effect of an additional volume of the backward aqueous phase, the AOT concentration factor needs to be analysed as it is believed to affect the volume changes of the phases. 
The additional volume of the backward aqueous phase, shown in Figure 5.3, increased when an AOT concentration of $>80 \mathrm{~g} / \mathrm{l}$ was used. However, the additional volume of the backward aqueous phase did not correspond to the mass of penicillin G. solubilised during backward extraction. It was found that the water which had been transferred in the reverse micelle during forward extraction was transferred back in the aqueous phase during backward extraction. Dungan et al. (1991) asserted that the water in the reverse micelle can be exchanged relatively quickly with the bulk water, but the release of biomolecules from the reverse micelle into the aqueous phase is slow and difficult due to the formation of aggregates between the biomolecule and surfactant.

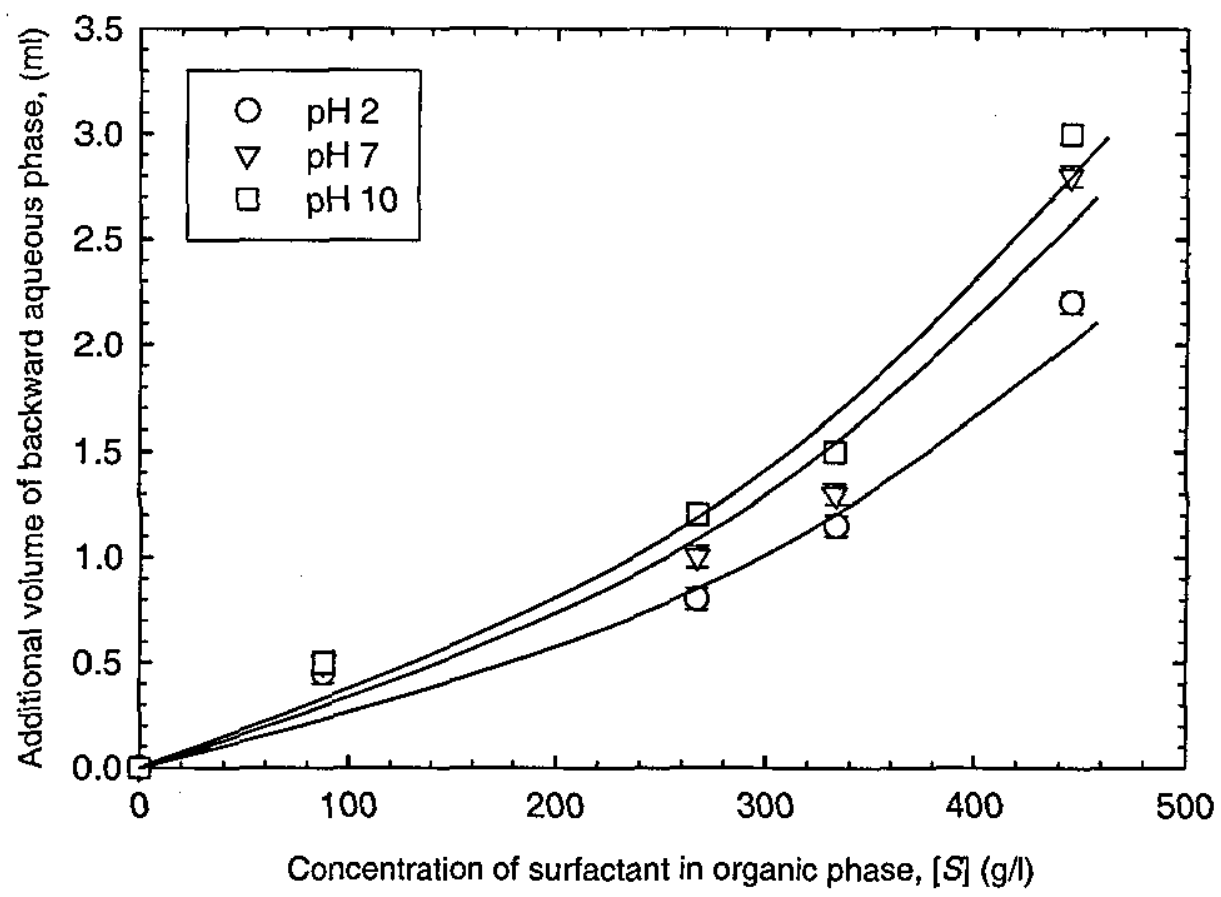

Figure 5.3: Additional volume of the backward aqueous phase at different surfactant concentrations. $\left([\mathrm{KCl}]=37 \mathrm{~g} / \mathrm{l}\right.$; temperature, $23 \pm 1^{\circ} \mathrm{C}$; stirring speed, $400 \mathrm{rpm}$ for 5 minutes; $\mathrm{pH} 7.6 \pm 0.5)$.

The increasing volume of the backward aqueous phase could reasonably be expected since the opposite phenomenon occurred during the forward extraction process. In the forward extraction of penicillin $\mathrm{G}$, since it is transferred from an aqueous to an organic phase, an increased volume of the reverse micelle organic phase occurred. 
Higher AOT concentration in the forward extraction promotes greater water transfer and it is not surprising that during backward extraction, the water transferred into the reverse micelle phase during forward extraction transferred back to the new aqueous phase. Figure 5.3 also shows that the additional volume changed slightly with $\mathrm{pH}$, except at lowest AOT concentration $(88 \mathrm{~g} / \mathrm{l})$. Further discussion on the effect of $\mathrm{pH}$ on the backward extraction is shown later in Section 5.2.3. Figure 5.4 shows the additional volume of the receiver phase during both forward and backward extraction. At all AOT concentrations and $\mathrm{pH}$ values, the additional volume in the forward extraction was transferred directly to the backward aqueous phase. The higher additional volume in the backward aqueous phase in comparison to the forward reverse micelle phase is probably due to solubilisation of the surfactant in the backward aqueous phase.

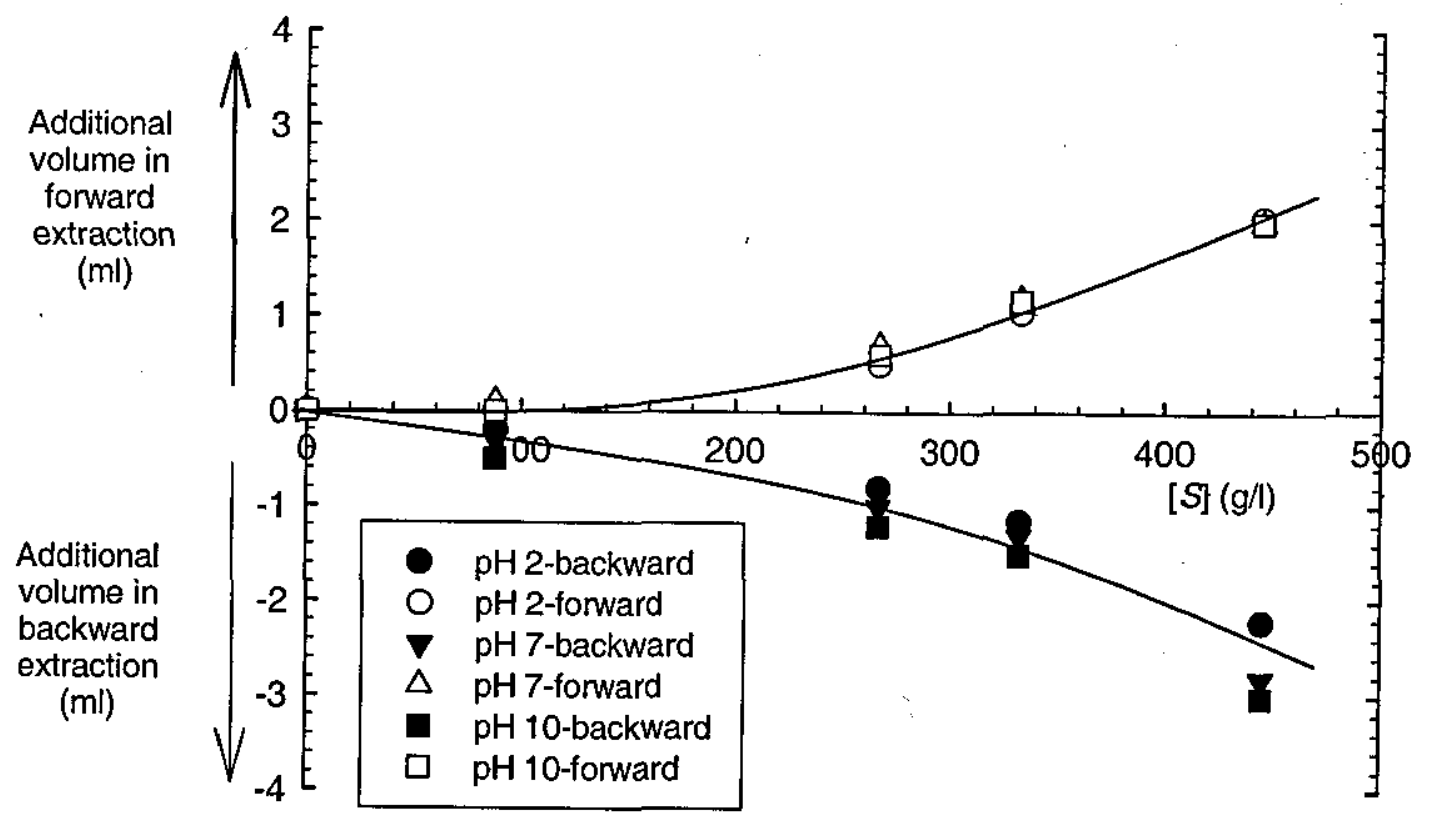

Figure 5.4: Overall volume changing during the forward and backward extraction processes.

\subsubsection{Effect of Salt Concentration}

Backward extraction of penicillin $G$ was carried out using different concentrations of $\mathrm{KCl}$ in a fresh aqueous phase at $\mathrm{pH} 7$. In order to investigate the effects of salt 
concentration, a reverse micelle phase containing penicillin $\mathrm{G}$ from the forward extraction was used. The initial mass of penicillin $\mathrm{G}$ in the reverse micelle phase before backward extraction $\left(P_{r m+o . i . b w}\right)$ was equivalent to the final mass of penicillin $\mathrm{G}$ in the reverse micelle phase after the forward extraction $\left(P_{r m+o f f f w}\right)$ which could be determined using equations (3.4)-(3.10). The initial concentration of penicillin $G$ in the aqueous phase before forward extraction, $[P]_{\text {aq.i.fw }}$, was fixed at $7.1 \mathrm{~g} / \mathrm{l}(35.6 \mathrm{mg})$, the AOT concentration was $88 \mathrm{~g} / \mathrm{l}$, and $\mathrm{pH}$ was 7.6. The initial mass of penicillin $\mathrm{G}$ in the reverse micelle before the backward extraction $\left(P_{r m+o . i . b w}\right)$ depended on the parameters used during the forward extraction. Table 5.1 shows that $P_{r m+o \text {.i.bw }}$ varies according to the parameters used. From equations (3.4)-(3.10), $P_{r m+o . i . b w}$ for Figure 5.5 is $29 \mathrm{mg}$.

Table 5.1: $P_{r m+o . i . b w}$ values at different parameters during forward extraction.

\begin{tabular}{|c|c|c|c|c|c|}
\hline $\mathrm{pH}$ & Salt type & {$[$ Salt $](\mathrm{g} / \mathrm{l})$} & {$[S](\mathrm{g} / \mathrm{l})$} & {$[P]_{\text {aq.i.fw }}(\mathrm{g} / \mathrm{l})$} & $P_{\text {m+o.i.bw }}(\mathrm{mg})$ \\
\hline 1.65 & $\mathrm{KCl}$ & 10 & 88 & 3.6 & 14.8 \\
1.65 & $\mathrm{KCl}$ & 10 & 88 & 7.1 & 30.7 \\
1.65 & $\mathrm{KCl}$ & 10 & 267 & 3.6 & 16.1 \\
1.65 & $\mathrm{KCl}$ & 10 & 267 & 7.1 & 31.8 \\
1.65 & $\mathrm{KCl}$ & 10 & 445 & 3.6 & 17.0 \\
1.65 & $\mathrm{KCl}$ & 10 & 445 & 7.1 & 33.5 \\
7.6 & $\mathrm{KCl}$ & 10 & 88 & 3.6 & 14.6 \\
7.6 & $\mathrm{KCl}$ & 10 & 88 & 7.1 & 29.0 \\
7.6 & $\mathrm{KCl}$ & 10 & 267 & 3.6 & 16.0 \\
7.6 & $\mathrm{KCl}$ & 10 & 267 & 7.1 & 31.2 \\
7.6 & $\mathrm{KCl}$ & 10 & 445 & 3.6 & 16.9 \\
7.6 & $\mathrm{KCl}$ & 10 & 445 & 7.1 & 33.2 \\
7.6 & $\mathrm{KCl}$ & 30 & 267 & 3.6 & 7.2 \\
7.6 & $\mathrm{CaCl}$ & 10 & 267 & 3.6 & 16.3 \\
7.6 & $\mathrm{CaCl}$ & 30 & 267 & 3.6 & 12.8 \\
\hline
\end{tabular}

Figure 5.5 shows that the solubilisation of penicillin $G$ in the backward aqueous phase depends on the salt concentration of the aqueous solution. The mass of penicillin $G$ in the backward aqueous phase increases with increasing salt concentration, because higher salt concentration leads to more favourable conditions for penicillin $G$ release from the reverse micelle and into the stripping solution. Zhang et al. (1999) asserted that the salt concentration of the backward aqueous phase can affect the efficiency of protein transfer (in their case) through the size exclusion effect. Moreover, as the salt concentration increases the electrostatic interaction between penicillin $\mathrm{G}$ molecules and AOT decreases. Consequently, the penicillin $\mathrm{G}$ molecule could be more readily released into the aqueous phase. 


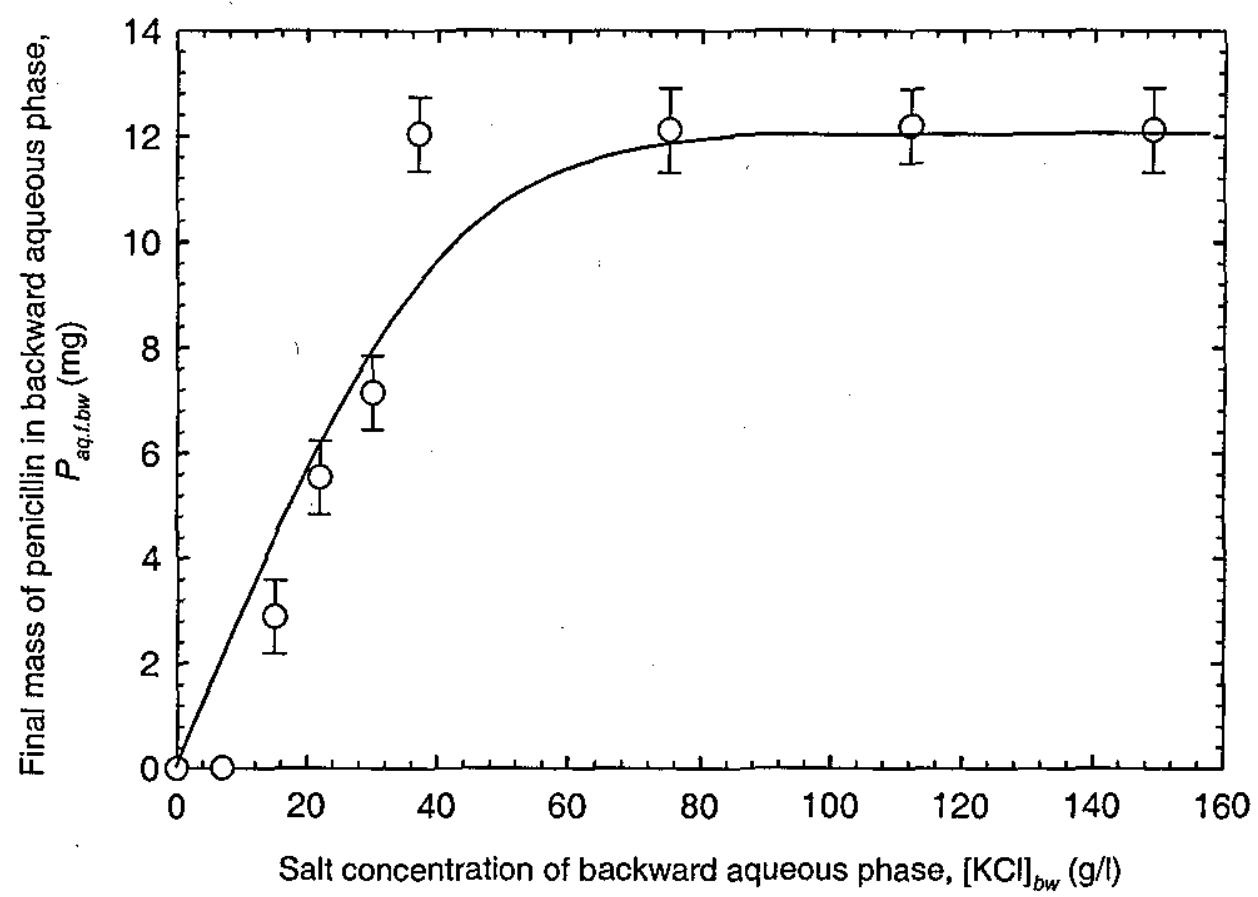

Figure 5.5: Effect of salt concentrations on the mass of penicillin $\mathrm{G}$ in the backward aqueous phase. (Temperature, $23 \pm 1^{\circ} \mathrm{C}$; stirring speed, $400 \mathrm{rpm}$ for 5 minutes; $\mathrm{pH} 7.6 \pm 0.5$; initial mass of penicillin $\mathrm{G}$ before backward extraction, $29 \mathrm{mg} ;[S]=88 \mathrm{~g} / \mathrm{l})$.

Shin et al. (2003) asserted that by increasing $\mathrm{KCl}$ concentration the concentration of chloride counterions increases, and as a result the competition with AOT for the positively charged biomolecule, as well as the dissociation of AOT anions from its counterions, would increase. As shown in Figure 5.5, as the concentration of $\mathrm{KCl}$ increased, so the mass of penicillin $\mathrm{G}$ in the aqueous phase, $P_{a q . j . b w}$, increased significantly. $\quad P_{a q f . b w}$ reached a plateau at a $\mathrm{KCl}$ concentration of about $37 \mathrm{~g} / 1$. Brandani et al. (1996), in their study of protein solubilisation, found that the salt concentration helped to release protein from the reverse micelle. In addition, solubilisation into the backward aqueous phase was much easier because the presence of ions reduced the electrostatic interaction of the protein-reverse micelle complex. Moreover, addition of ions prevented biomolecule-surfactant precipitation at the interface. 


\subsubsection{Effect of $\mathrm{pH}$}

The $\mathrm{pH}$ of the backward aqueous phase must be such that the biomolecule has the same charge as the surfactant molecules, so that repulsion forces are created, which causes the release of the biomolecule from the reverse micelle (Leser and Luisi, 1990; Brandani et al., 1996). Ono et al. (1996) concluded that even though the addition of alcohol promotes haemoglobin release from reverse micelle, $\mathrm{pH}$ is a more important factor in the backward extraction process. They found that at $\mathrm{pH}>\mathrm{pI}$ of haemoglobin, the backward extraction yield was remarkably enhanced regardless of how much alcohol was added to the system. Figure 5.6 presents results for the mass of penicillin $\mathrm{G}$ in the backward aqueous phase $\left(P_{a q . f b w}\right)$, with three different AOT concentrations, as a function of $\mathrm{pH}$. The mass of penicillin $\mathrm{G}$ in the aqueous phase is almost constant at a fixed $\mathrm{pH}$ value, and only differs for different AOT concentrations. The pI of the penicillin $\mathrm{G}$ molecule is low at 2.7. At a pH higher than 2.7 , the penicillin $G$ surface has a greater number of negative charges, which reduces the penicillin G-reverse micelle interaction. The positive charge of both penicillin $G$ and reverse micelle molecules facilitated penicillin $G$ transfer out of the reverse micelle. This explains why the backward extraction process of penicillin $\mathrm{G}$ could be performed over a wide range of $\mathrm{pH}$.

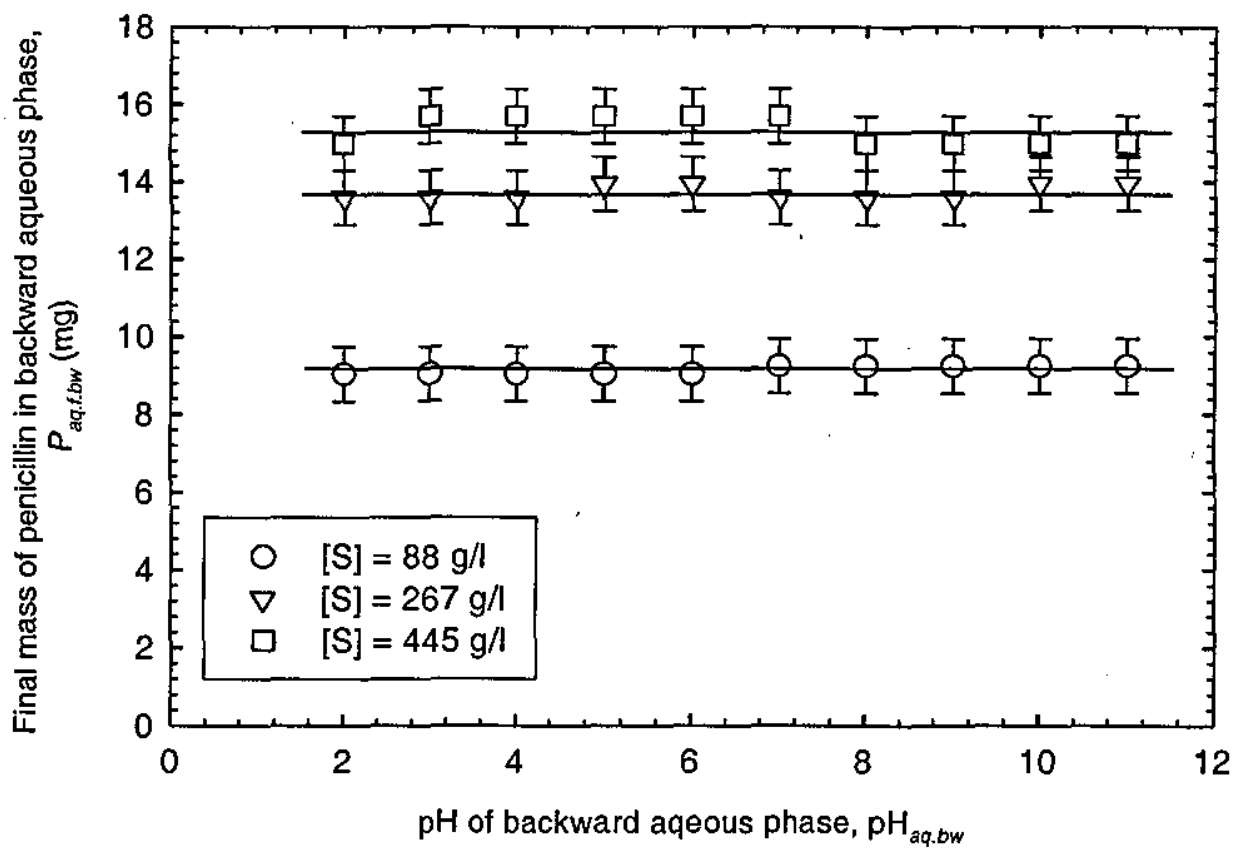

Figure 5.6: Effect of $\mathrm{pH}$ on the mass of penicillin $\mathrm{G}$ in the backward aqueous phase. $([\mathrm{KCl}]=$ $37 \mathrm{~g} / \mathrm{l}$; temperature, $23 \pm 1^{\circ} \mathrm{C}$; stirring speed, $400 \mathrm{rpm}$ for 5 minutes). 


\subsubsection{Effect of Initial Mass of Penicillin $G$ and Surfactant Concentration}

To assess the influence of backward extraction experiments were carried out by contacting the reverse micelle solution from forward extraction with the backward aqueous phase containing $1 \mathrm{M} \mathrm{KCl}$ solutions at a volume ratio of $1: 1$. The reverse micelle solutions from forward extraction containing various mass of penicillin $G$ resulted from different AOT concentrations used during forward process. Figure 5.7 shows that the final mass of penicillin $\mathrm{G}$ in the backward aqueous phase $\left(P_{a q . f . b w}\right)$ depends on the initial concentration of penicillin $\mathrm{G}$ before forward extraction $\left(P_{\text {aq.i.fw }}\right)$ and more importantly depends on AOT concentration used during forward extraction process. The solubilisation of penicillin $\mathrm{G}$ in the backward aqueous phase increased when the AOT concentration was increased. This is because during forward extraction, the solubilisation of penicillin $G$ was accompanied by an additional volume of reverse micelle phase; higher AOT concentration led to more additional volume. Before the backward extraction process, the system with higher AOT concentration contained more water than the system with lower AOT concentration. As previously discussed, the backward extraction is also affected by osmotic pressure, the gravitational force, and the nature of penicillin $\mathrm{G}$ molecule which is hydrophilic and interfacially active. As a result of all these factors, a system with higher amount of water seems to more be able to release greater mass of penicillin $\mathrm{G}$. These results show that, besides salt concentration and $\mathrm{pH}$, a higher AOT concentration during forward extraction is also important in order to achieve better solubilisation in the backward extraction. 


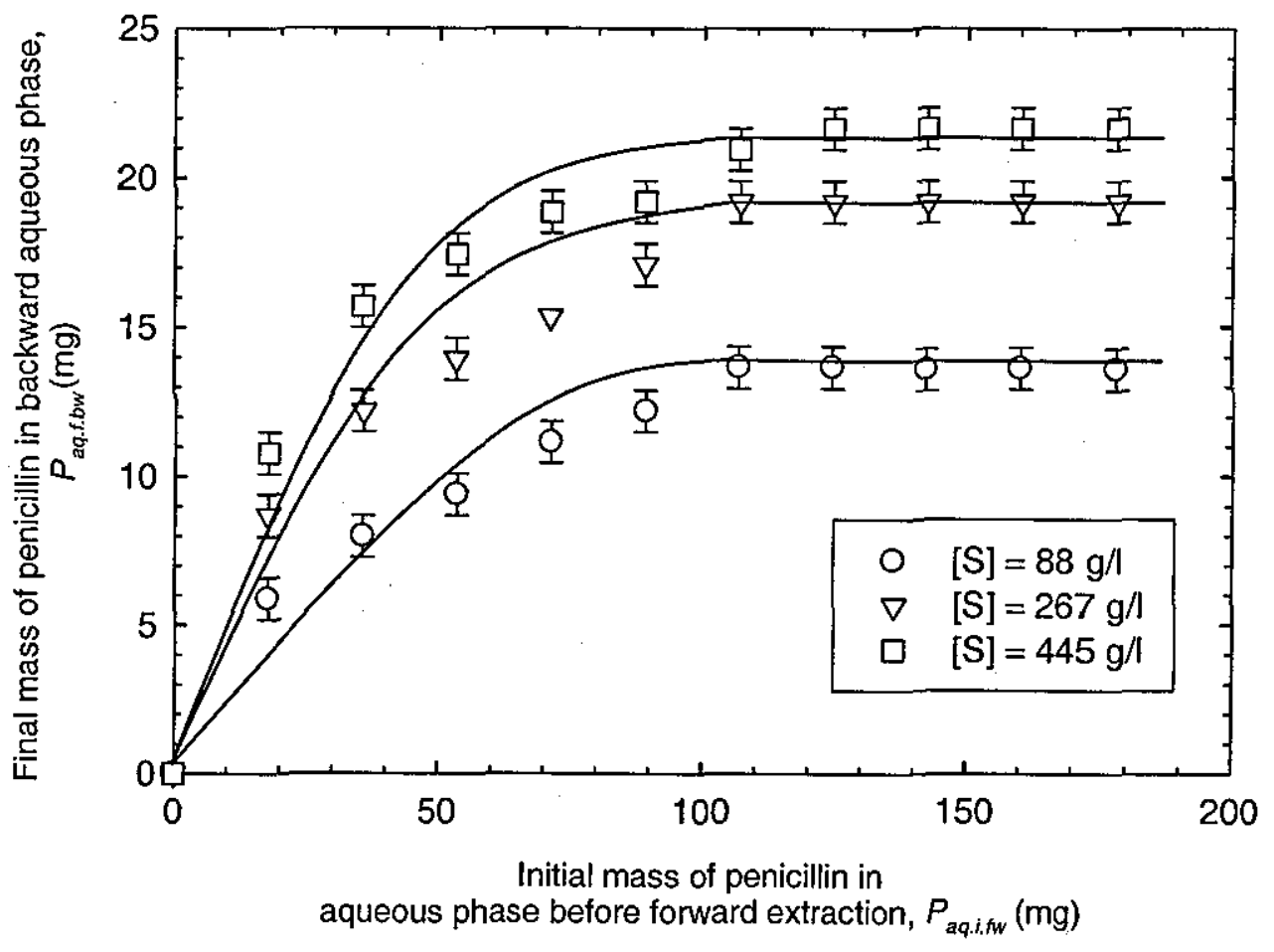

Figure 5.7: Effect of initial mass of penicillin $\mathrm{G}$ before forward extraction to the mass of penicillin $\mathrm{G}$ in the backward aqueous phase. (Forward conditions: $[\mathrm{KCl}]=7 \mathrm{~g} / \mathrm{l}$; temperature, $23 \pm 1^{\circ} \mathrm{C}$; stirring speed, $400 \mathrm{rpm}$ for 10 minutes; $\mathrm{pH} 7.6 \pm 0.5$. Backward conditions: $[\mathrm{KCl}]=$ $37 \mathrm{~g} / \mathrm{l}$; temperature, $23 \pm 1^{\circ} \mathrm{C}$; stirring speed, $400 \mathrm{rpm}$ for 5 minutes; $\mathrm{pH} 7.6 \pm 0.5$ ).

Figure 5.7 shows that $P_{\text {aq.f.bw }}$ moves toward a constant value as $P_{\text {aq.i.fw }}$ is increased. Due to the hydrophobic interactions between AOT molecules the reverse micelle have a tendency to move to the interface of the organic and aqueous phases. During mixing, a dynamic equilibrium occurs in the solution, which encourages the renewal of AOT molecules at the interface. Some of the AOT molecules at the interface interact with water to form micelles in the aqueous phase and at the same time these molecules release the penicillin $G$. The renewal process at the interface continues until there are no more reverse micelle in the organic phase that could move to the interface and there are no more penicillin $\mathrm{G}$ molecules released in the aqueous phase. This is the reason why $P_{a q . f b w}$ goes to a constant value as $P_{a q . i . f w}$ is increased.

The effect of the initial mass of penicillin $\mathrm{G}$ before backward extraction $\left(P_{r m+o . i . b w}\right)$ was investigated and the results are shown in Figure 5.8. Results showed that the backward extraction was significantly affected by the $P_{r m+o . i . b w}$. From the forward 
extraction, the highest mass of penicillin $\mathrm{G}$ solubilised into reverse micelle was at [S] $=267 \mathrm{~g} / \mathrm{l}$.

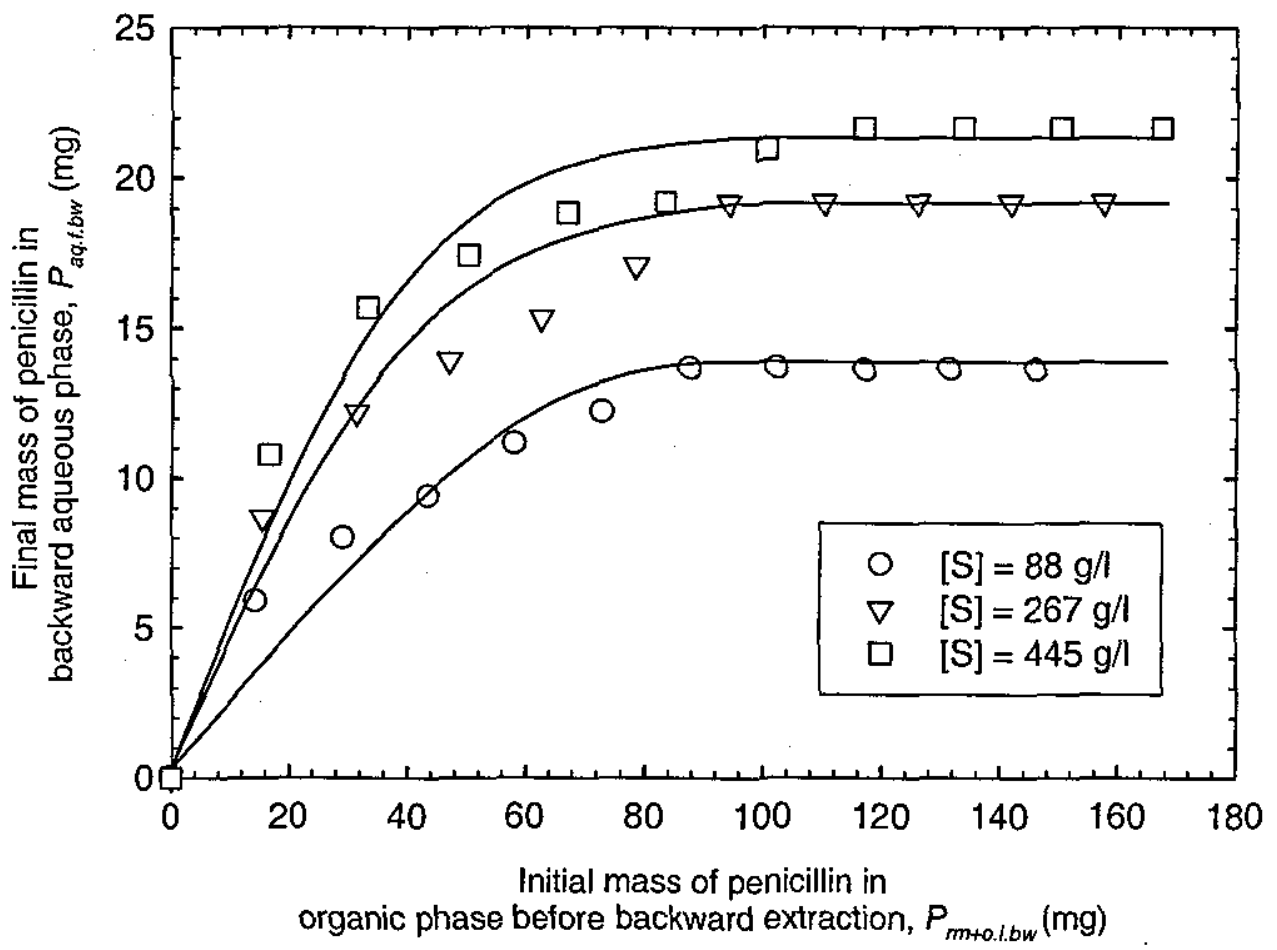

Figure 5.8: Effect of initial mass of penicillin $\mathrm{G}$ before backward extraction to the mass of penicillin $\mathrm{G}$ in the backward aqueous phase. $\left([\mathrm{KCl}]=37 \mathrm{~g} / 1\right.$; temperature, $23 \pm 1^{\circ} \mathrm{C}$; stirring speed, 400 rpm for 5 minutes; $\mathrm{pH} 7.6 \pm 0.5$ ).

However, after backward extraction the highest mass of penicillin $G$ in the aqueous phase was obtained at $[\mathrm{S}]=445 \mathrm{~g} / \mathrm{l}$. Lee et al. (2004) confirmed that denaturation of biomolecules during backward extraction can be avoided by using high salt and surfactant concentrations. This may explain why the concentration of penicillin $\mathrm{G}$ in backward extraction increases by increasing the surfactant concentration even though $[P]_{r m+o f f w}$ is about the same for $[S]=267 \mathrm{~g} / 1$ and $445 \mathrm{~g} / \mathrm{l}$ as shown in Figure 4.8 .

Figure 5.9 represents the overall process of penicillin $\mathrm{G}$ extraction, which is from the initial mass of penicillin $\mathrm{G}$ in the aqueous phase before forward extraction $\left(P_{\text {aq.i.fw }}\right)$ to the final mass of penicillin $G$ in the aqueous phase after backward extraction $\left(P_{a q f . b w}\right)$. The nearer the plot to the $45^{\circ}$ line indicates better overall process as it shows the mass of penicillin $G$ transferred in the backward aqueous phase is the 
same as the initial mass at the beginning the process before forward extraction. The results in Figure 5.9 demonstrate that at all tested AOT concentrations the plot is far from the $45^{\circ}$ line, and suggests that the overall extraction process of penicillin $G$ is relatively complex despite promising results in the forward process.

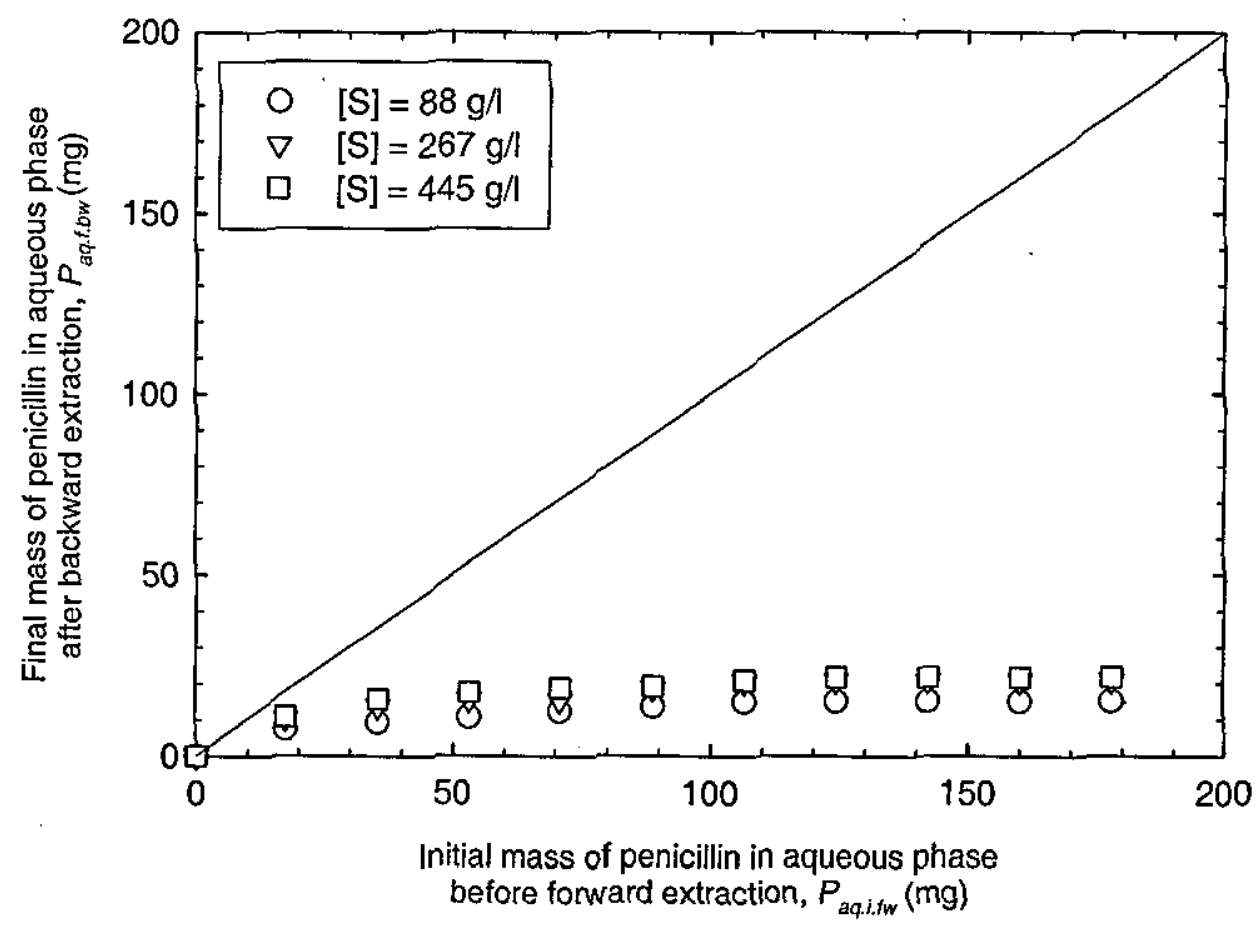

Figure 5.9: Overall extraction of penicillin $\mathrm{G} .\left([\mathrm{KCl}]=37 \mathrm{~g} / \mathrm{l}\right.$; temperature, $23 \pm 1^{\circ} \mathrm{C}$; stirring speed, $400 \mathrm{rpm}$ for 5 minutes; $\mathrm{pH} 7.6 \pm 0.5$ ).

Removal efficiency can be used to calculate the overall effectiveness of the extraction process, which is defined as:

$$
\% \text { Removal efficiency }=\frac{P_{a q, f . b w}}{P_{a q, i f w}} 100 \%
$$

where $P_{\text {aqf.f. }}$ is the final mass of penicillin $\mathrm{G}$ in the aqueous phase after backward extraction (mg) and $P_{a q . i f w}$ the initial mass of penicillin $\mathrm{G}$ before forward extraction (mg).

Figure 5.10 shows that removal efficiency decreased when $P_{a q . i . f w}$ increased for all three $[S]$ of 88,267 and $445 \mathrm{~g} / \mathrm{l}$. The highest removal efficiency percentage (60\%) was obtained at $P_{a q . i . f w}=17.6 \mathrm{mg}$ and $[S]=445 \mathrm{~g} / \mathrm{l}$. 


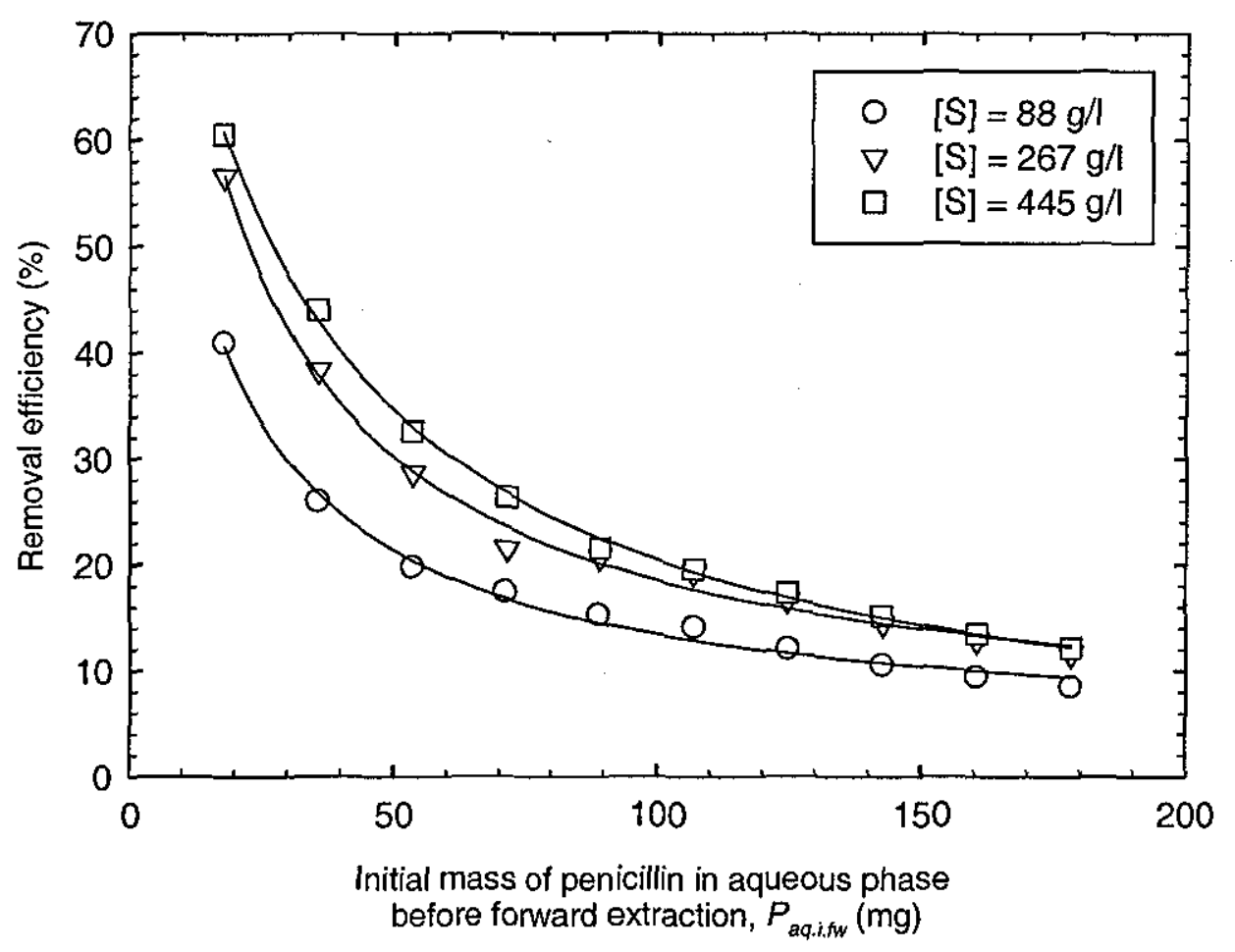

Figure 5.10: Removal efficiency of penicillin $\mathrm{G}$ at different AOT concentrations.

\subsection{Thermodynamic Framework for Solubilisation}

In the backward extraction process the dependency of the distribution coefficient on penicillin concentration, AOT concentration, and $\mathrm{pH}$ is measured. Although a similar model to forward extraction is used in the backward extraction the parameters need to be redefined appropriately. For example, equation (4.4) defined the forward extraction distribution coefficient, $K_{f w}$, as:

$$
K_{f w}=\frac{[P]_{r m+o . f . f w}}{[P]_{a q . f . f w}}
$$

Equation 4.4 can be used only for forward extraction process due to the solubilisation of penicillin $\mathrm{G}$ from an aqueous phase to an organic phase containing reverse micelle. By contrast, in the backward extraction the penicillin G moves (solubilises) 
from an organic phase to an aqueous phase, and the distribution coefficient of backward extraction $\left(K_{b w}\right)$ is expressed as follows:

$$
K_{b w}=\frac{[P]_{a q . f . b w}}{[P]_{r m+o . f . b w}}
$$

where $[P]_{a q . f . b w}$ is the final concentration of penicillin $\mathrm{G}$ in the aqueous phase after backward extraction and $[P]_{r m+o . f . b w}$ the final concentration of penicillin $G$ in the reverse micelle organic phase after backward extraction.

A higher value of $K_{b w}$ indicates better solubilisation of penicillin $\mathrm{G}$ into the backward aqueous phase. When the backward extraction distribution coefficient, $K_{b w}$, is plotted against the concentration of penicillin $G$ in the backward reverse micelle $\left([P]_{r m+o f . b w}\right)$, with all other parameters being held constant and the experimental data extrapolated back to $[P]_{r m+o f . b w}=0$, a value for the distribution coefficient at infinite dilution for backward extraction $\left(K_{E . b w}^{\infty}\right)$ is found. Following this procedure, the results for $\mathrm{pH} 1.65$ and 7.6 are shown in Figure 5.11.

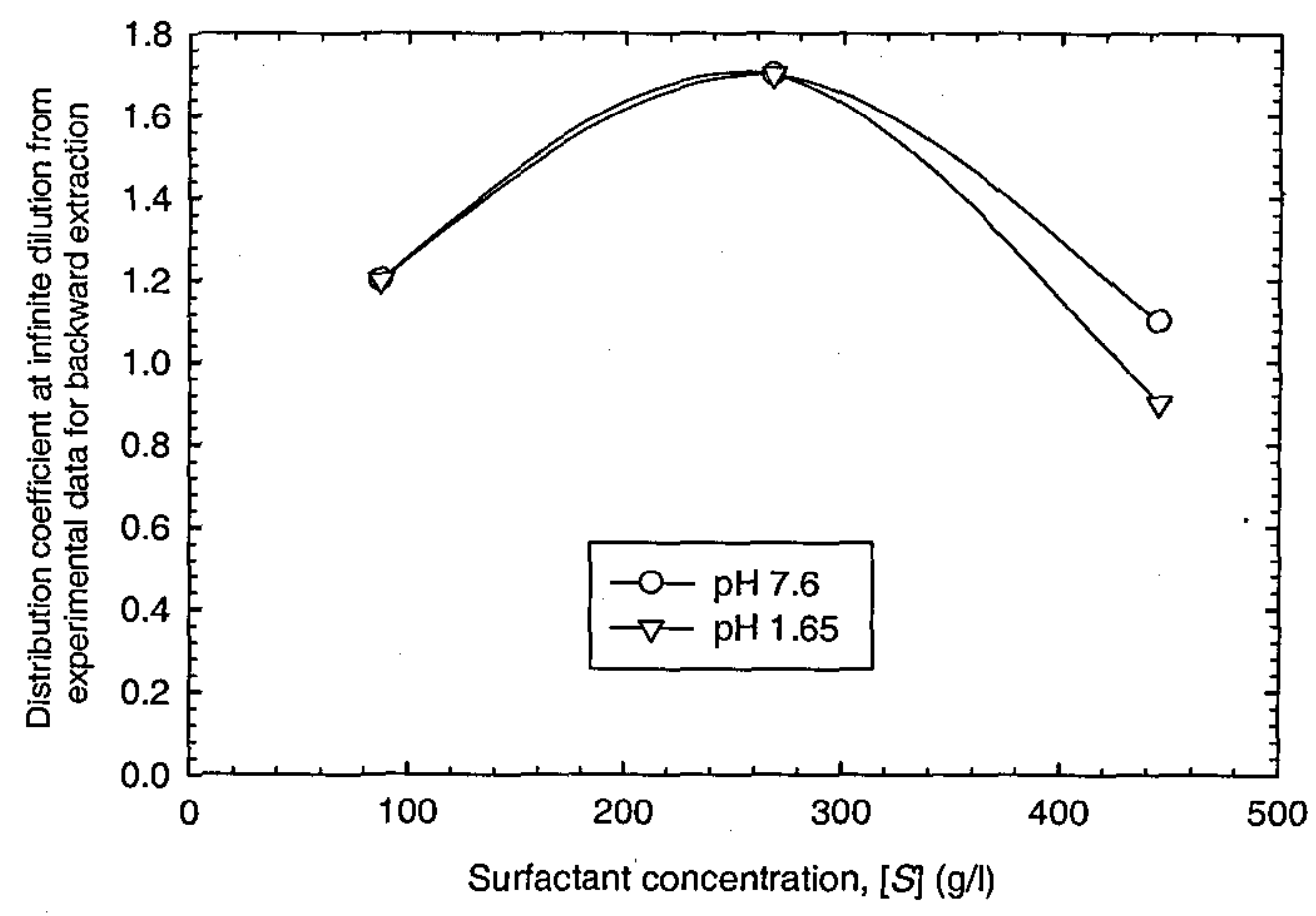

Figure 5.11: Variation of distribution coefficient at infinite dilution from experimental data for backward extraction ( $K_{E . b w}^{\infty}$ ) with AOT concentration and $\mathrm{pH}$. 
The backward extraction distribution coefficient at infinite dilution $\left(K_{E b w}^{\infty}\right)$ depends markedly on surfactant concentration and almost is independent of pH. $K_{E . b w}^{\infty}$ is similar for both $\mathrm{pH}$ values except at high surfactant concentrations. $K_{E . b w}^{\infty}$ increases as the surfactant concentration increases up to $267 \mathrm{~g} / \mathrm{l}$, however, $K_{E . b w}^{\infty}$ decreases when surfactant concentration is raised above $267 \mathrm{~g} / \mathrm{l}$. Regarding the result obtained from forward extraction, the solubilisation of penicillin in the reverse micelle was higher at low pH for all surfactant concentrations. Figures 4.13 and 5.11 show that $K_{f w}^{\infty} \neq 1 / K_{b w}^{\infty}$, as discussed toward the end of Section 5.3.1.2.

\subsubsection{Distribution Coefficient at Infinite Dilution $\left(K_{b w}^{\infty}\right)$}

Similar to forward extraction, the Langmuir and Brandani models were used to find the dependency of the parameters used during backward extraction. Experimental results from the backward extraction are reported in Tables A5-1 and A5-2 as shown in Appendix 5. The final concentration of penicillin $G$ in the backward reverse micelle (organic) phase $\left([P]_{r m+o f . b w}\right)$ and the final concentration of penicillin $\mathrm{G}$ in the backward aqueous phase $\left([P]_{\text {aq.f.bw }}\right.$ ) were measured at surfactant concentrations, $[S]$, $88 \mathrm{~g} / \mathrm{l}, 267 \mathrm{~g} / \mathrm{l}$ and $445 \mathrm{~g} / \mathrm{l}$. Two different $\mathrm{pH}$ values, 7.6 and 1.65 were considered for each value of surfactant concentration. The models described in Section 5.3.1.1 and 5.3.1.2 were fitted to each set of experimental data.

The equations from both Langmuir and Brandani models in the Chapter 4 specifically can be used only for forward extraction, and in the backward extraction these equations have been adapted to the solubilisation process of penicillin $G$ from the reverse micelle to an aqueous phase.

\subsubsection{Data Fitted with the Langmuir Model}

The Langmuir model for solubilisation of penicillin $\mathrm{G}$ in the backward extraction process is given by equation: 


$$
[P]_{a q . f . b w}=\frac{a_{L . b w}[P]_{r m+o . f . b w}}{1+b_{L . b w}[P]_{r m+o . f . b w}}
$$

where $a_{L, b w}$ and $b_{L, b w}$ are adjustable parameters.

The distribution coefficient of Langmuir model for backward extraction can be written as:

$$
K_{L . b w}=\frac{[P]_{a q . f . b w}}{[P]_{r m+o . f . b w}}=\frac{a_{L . b w}}{1+b_{L . b w}[P]_{r m+0 . f . b w}}
$$

At infinite dilution, when final concentration of penicillin $G$ in the reverse micelle organic phase for backward extraction $\left([P]_{r m+o . f . b w}\right)$ goes to zero, the distribution coefficient is:

$$
K_{L . b w}^{\infty}=a_{L . b w}
$$

where $K_{L . b w}^{\infty}$ is the distribution coefficient of the Langmuir model at infinite dilution for backward extraction.

By using the SigmaPlot curve fitting routines, equation (5.3) was fitted to experimental data obtained in the backward extraction process. Figure 5.12 shows comparison between experimental data and the fitted models at $\mathrm{pH} 7.6$ and $[\mathrm{S}]=88$ $\mathrm{g} / \mathrm{l}$ (other results for different $\mathrm{pH}$ and $[S]$ were shown in Figures A5.2-1 - A5.2-5 in Appendix 5). From the results, $K_{L . b w}^{\infty}\left(\right.$ for the Langmuir model: $\left.K_{L . b w}^{\infty}=a_{L . b w}\right)$ and $b_{L b w}$ values were obtained for both pH's as shown in Figure 5.13. Figure 5.13 shows that by increasing [S] up to $267 \mathrm{~g} / 1, K_{L . b w}^{\infty}$ increases significantly for $\mathrm{pH} 1.65$, whilst it increases slightly for $\mathrm{pH} 7.6 . K_{E . b w}^{\infty}$ decreases when $[S]$ increases above $267 \mathrm{~g} / \mathrm{l}$ for both $\mathrm{pH}$ values. By comparing data at each $\mathrm{pH}, K_{E . b w}^{\infty}$ is significantly higher at $\mathrm{pH}$ 1.65 than at $\mathrm{pH} 7.6$ for all $[S]$. There is a weak relationship between $K_{L . b w}^{\infty}$ and $[S]$ and $\mathrm{pH}$ at $\mathrm{pH}$ 7.6. Figure 5.13 also shows that $b_{L . b w}$ depends on both $\mathrm{pH}$ and $[S]$. 


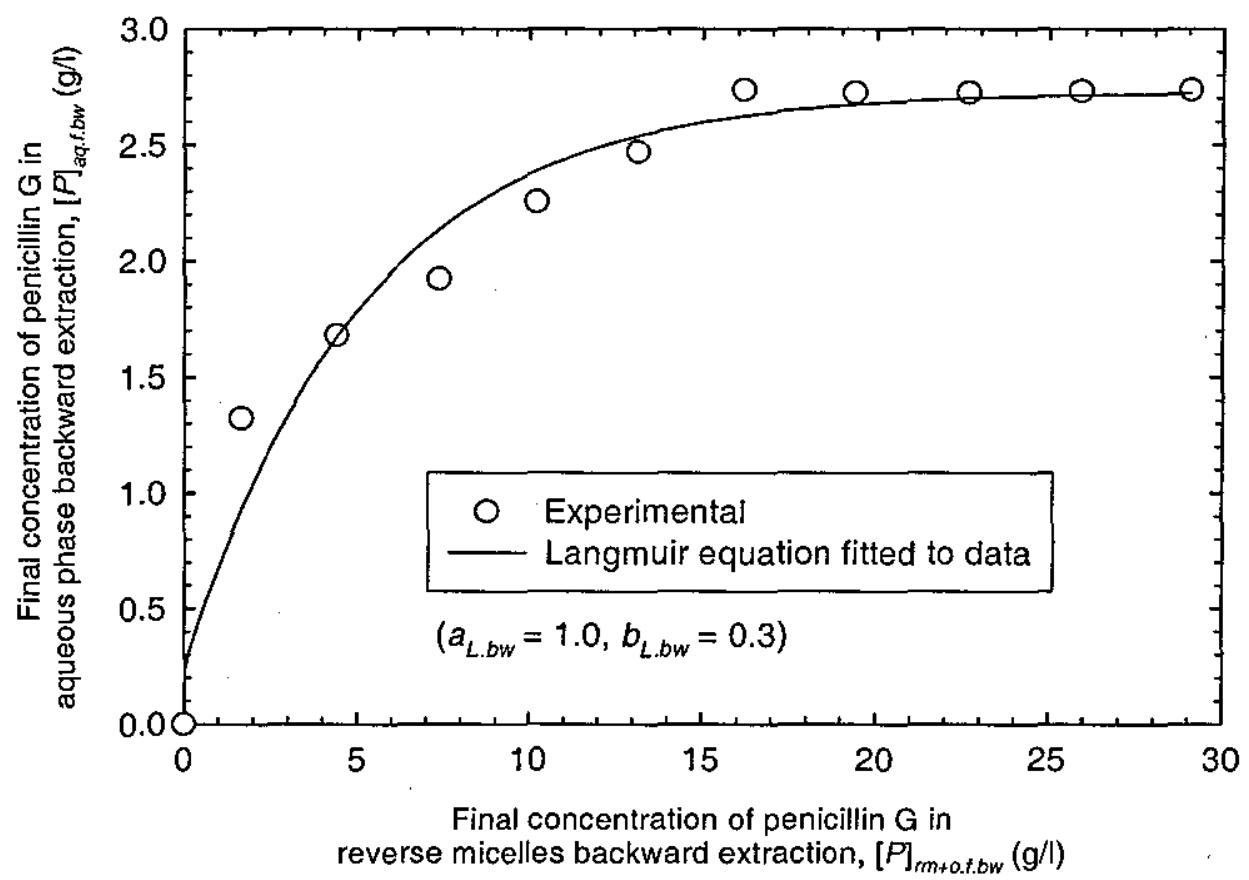

Figure 5.12: Langmuir model fitted to experimental data at $\mathrm{pH} 7.6$ and $[S]=88 \mathrm{~g} / 1$ (backward extraction).

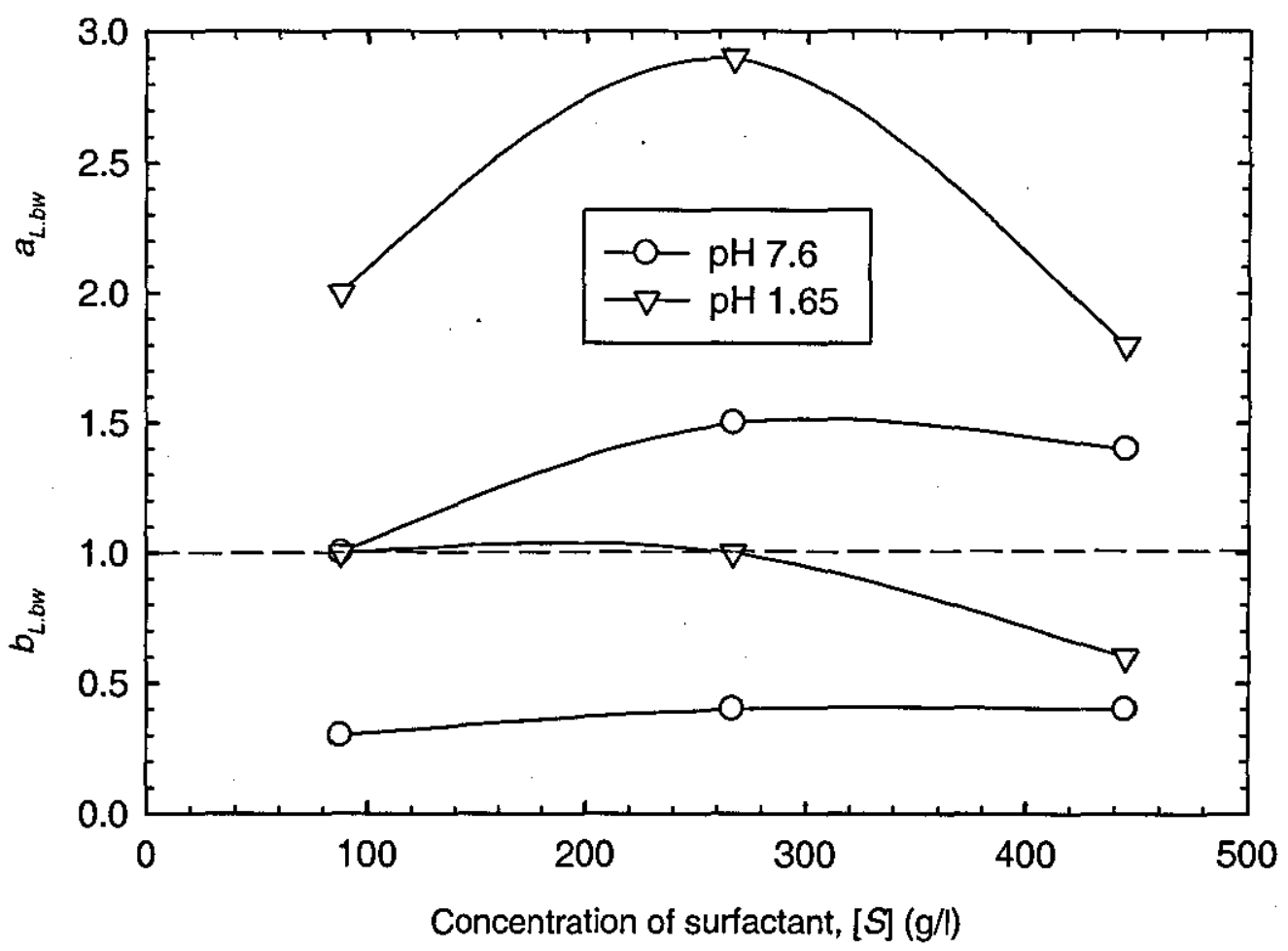

Figure 5.13: Partition coeffiecient at infinite dilution of Langmuir model for backward extraction, $a_{L, b w}\left(=K_{L . b w}^{\infty}\right)$ and $b_{L . j w}$ values at different $\mathrm{pH}$ and surfactant concentration. 


\subsubsection{Data Fitted with the Brandani ModeI}

The final concentration of solute in the backward aqueous phase can be expressed as:

$$
[P]_{a q . f . b w}=\frac{a_{B . b w} b_{B . b w}[S][P]_{r m+o . f . b w}}{1+b_{B . b w}[P]_{m+o . f . b w}}
$$

where $a_{B . b w}$ and $b_{B . b w}$ are adjustable parameters; $b_{B . b w}$ is a parameter characteristic of the inner surface of reverse micelle, the solute, and the temperature. Using this equation, the distribution coefficient from the Brandani model for backward extraction $\left(K_{B . b w}\right)$ depends on both $[S]$ and $[P]_{r m+o f . b w}$ and can be written as:

$$
K_{B . b w}=\frac{[P]_{a q . f . b w}}{[P]_{r m+o . f . b w}}=\frac{a_{B . b w} b_{B . b w}[S]}{1+b_{B . b w}[P]_{r m+o . f . b w}}
$$

At infinite dilution $[P]_{r m+o f . b w f} \rightarrow 0$ and

$$
K_{B . b w}^{\infty}=a_{B . b w} b_{B . b w}[S]
$$

or

$$
\ln K_{B . b w}^{\infty}=\ln a_{B . b w}+\ln b_{B . b w}+\ln [S]
$$

where $K_{B . b w}^{\infty}$ is the distribution coefficient of the Brandani model at infinite dilution for backward extraction.

The Brandani model was fitted to the experimental data using Equations (5.6). Figure 5.14 shows a good agreement between experimental data from the backward extraction and the Brandani model fitted to experimental data (see Figures A5.3-1 A5.3-5 in Appendix 5 for other fitted results at different $\mathrm{pH}$ and $[S])$. 


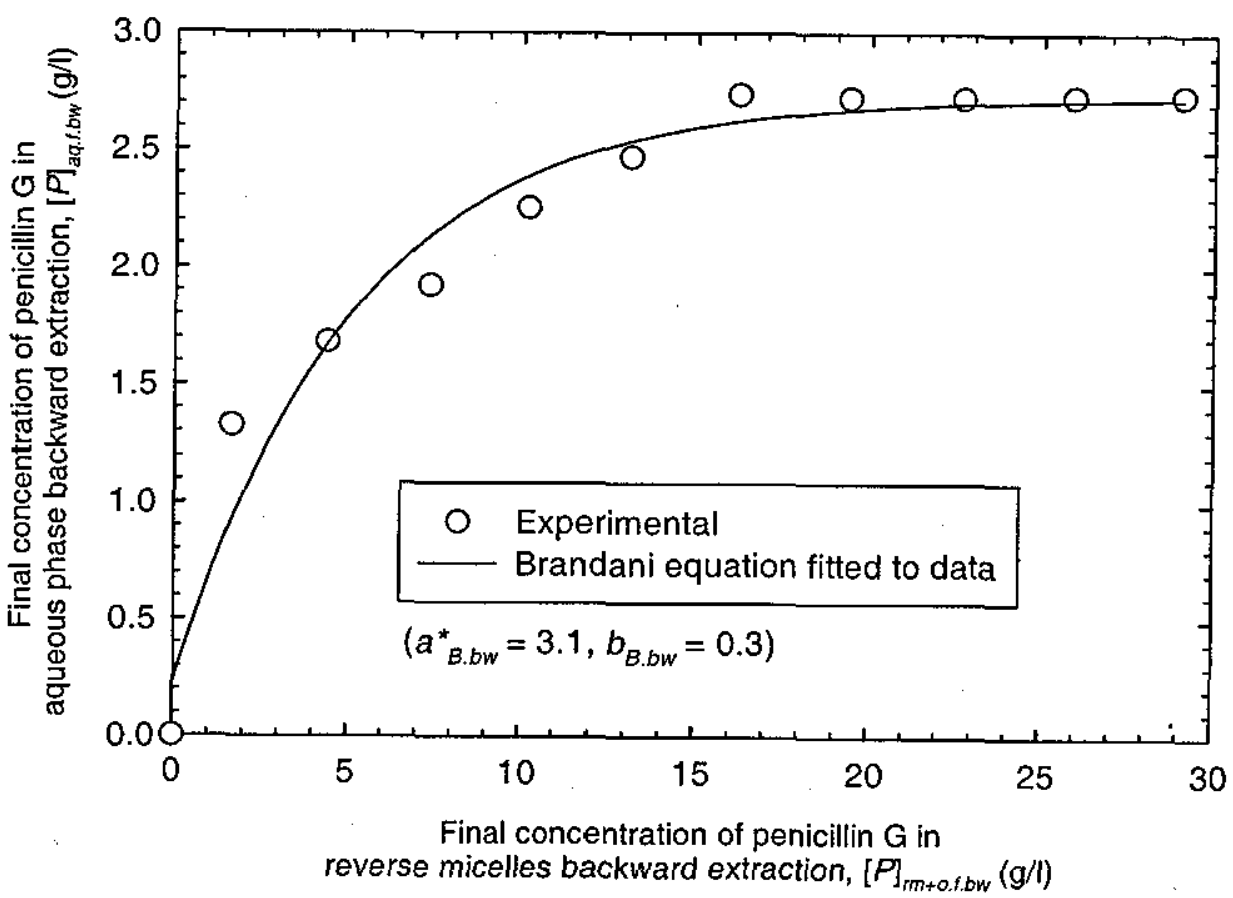

Figure 5.14: Brandani model fitted to experimental data at $\mathrm{pH} 7.6$ and $[S]=88 \mathrm{~g} / \mathrm{l}$ for backward extraction. $\left(a_{B . b w}=a_{B . b w}[S]\right)$.

$a_{B . b w}$ and $b_{B . b w}$ are the parameters from the fitting; $a_{B . b w}$ is dimensionless and $b_{B . b w}$ has the units $(1 / \mathrm{g})$. Similar to forward extraction, the distribution coefficient at infinite dilution from the Brandani model for backward extraction $\left(K_{B . b w}^{\infty}\right)$ considered both $a_{B . b w}$ and $b_{B . b w}$ values as shown in equation (5.7); therefore $b_{B . b w}$ values have been taken into account to verify if there is any significant effect on $\mathrm{pH}$ and/or $[S]$. Figure 5.15 shows the behaviour of the partition coefficient at infinite dilution of the Brandani model for backward extraction $\left(K_{B . b w}^{\infty}\right)$ calculated as a function of $\mathrm{pH}$ and surfactant concentration. The results show that $K_{B . b w}^{\infty}$ increases with increasing $[S]$ up to $267 \mathrm{~g} / 1$ for both $\mathrm{pHs}$, and leveled off when [S] increased above $267 \mathrm{~g} / 1$. $K_{B . b w}^{\infty}$ is found to be dependent on $[S]$ for both $\mathrm{pH}$ values. Similar to forward extraction, the Brandani model was found to be more relevant compared to the Langmuir model because this model describes the thermodynamic distribution for both the interfacial phase and in the reverse micelle phase. Figure 5.15 shows that $b_{B \cdot b w}$ has not significantly change with $\mathrm{pH}$ at higher value of $\mathrm{pH}$. For $\mathrm{pH} 1.65$, there is a significant decrease of $b_{B \cdot \text { bw }}$ when [S] increases. 


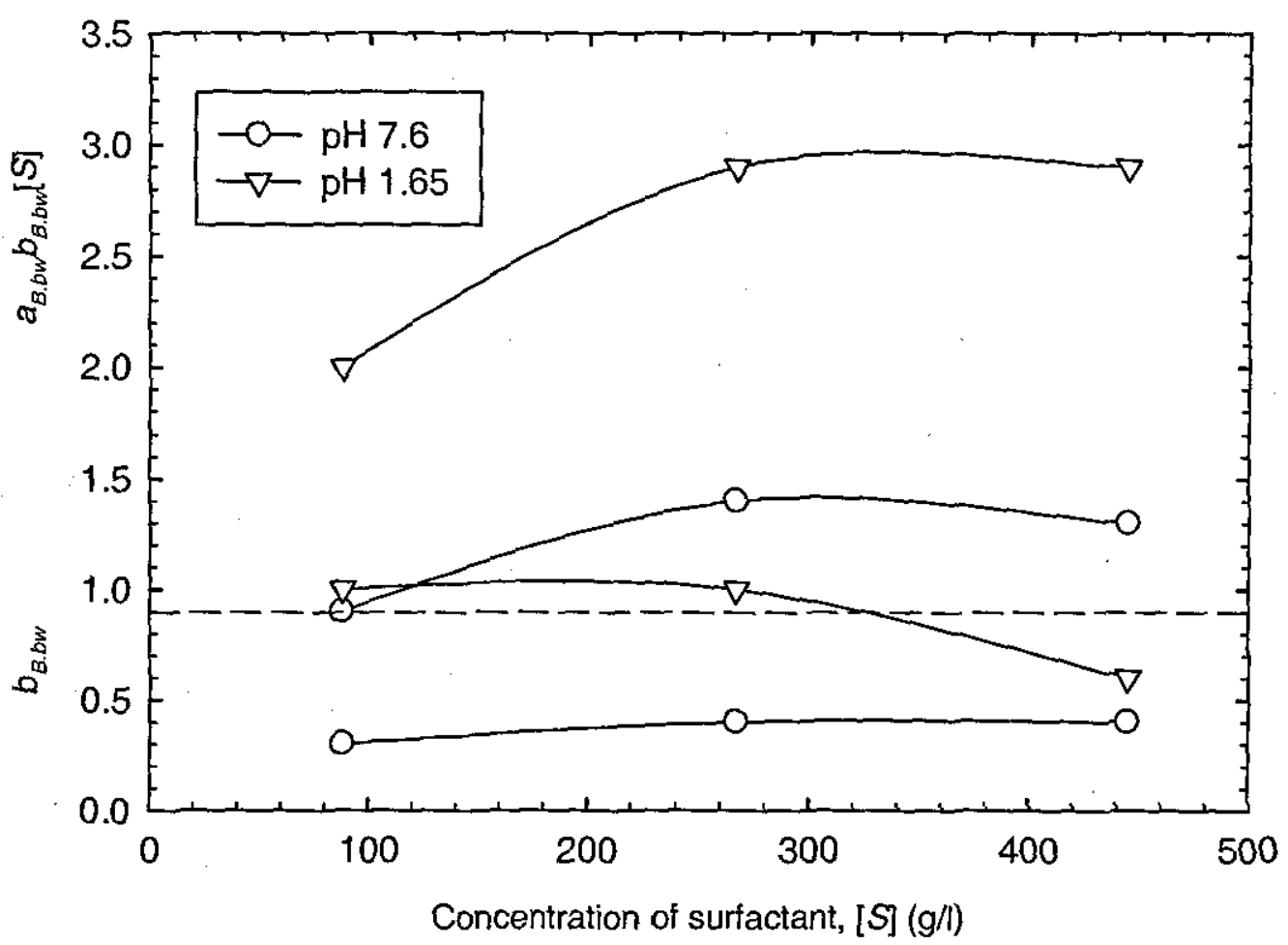

Figure 5.15: Distribution coefficient at infinite dilution of Brandani model for backward extraction, $K_{B . b w}^{\infty}\left(=a_{B . b w} b_{B . b w}[S]\right)$ and $b_{B . b w}$ values at different $\mathrm{pH}$ and surfactant concentration, $[S]$.

The distribution coefficient at infinite dilution for backward extraction, $K_{b w}^{\infty}$, from the experimental data $\left(K_{E . b w}^{\infty}\right)$, the Langmuir model $\left(K_{L . b w}^{\infty}\right)$, and the Brandani model $\left(K_{B . b w}^{\infty}\right)$ are shown in Table 5.2.

Table 5.2: Comparison of distribution coefficient at infinite dilution for backward extraction $\left(K_{b w}^{\infty}\right)$ between experimental results, Langmuir model and Brandani model.

\begin{tabular}{|c|c|c|c|c|}
\hline \multicolumn{2}{|c|}{ Experimental parameters } & $K_{E . b w}^{\infty}$ & $K_{L . b w}^{\infty}$ & $K_{B . b w}^{\infty}$ \\
\hline$[\mathrm{S}]$ & $\mathrm{pH}$ & 1.1 & 1.0 & 0.9 \\
\hline 88 & 7.6 & 1.6 & 1.5 & 1.4 \\
445 & 7.6 & 1.1 & 1.4 & 1.3 \\
88 & 7.6 & 1.0 & 2.0 & 2.0 \\
267 & 1.65 & 1.5 & 2.9 & 2.9 \\
445 & 1.65 & 0.9 & 1.8 & 2.9 \\
\hline
\end{tabular}


As discussed previously in Chapter 4, the distribution coefficient at infinite dilution of forward extraction $\left(K_{f w}^{\infty}\right)$ for experimental, Langmuir and Brandani show similarity which indicates that both models fit well to the experimental data. By contrast, for the backward extraction as shown in Table 5.2, the values of $K_{E . b w}^{\infty}$ for both models do fit well to the experimental data.

During forward extraction, without a mixing process, the penicillin $\mathrm{G}$ molecules move through the bulk aqueous phase by diffusion. However, through the diffusion process, only a small mass of the molecules move to the interface, and attach to the negatively charged AOT molecules, while most of the penicillin G molecules stay in the aqueous phase. Penicillin $\mathrm{G}$ is an interfacial active component that is attracted to AOT molecules (as discussed in Section 4.3), and attaches to the AOT molecules due to hydrophobic interactions between both molecules. These molecules solubilise in the bulk organic phase and simultaneously form reverse micelle which contain penicillin $\mathrm{G}$ surrounded by a water pool. When mixing is applied to the system, the aqueous phase containing penicillin $\mathrm{G}$ is transported by convection through the bulk aqueous phase. Mixing is crucial in reverse micelle formation and more reverse micelle formation means more penicillin $\mathrm{G}$ can be solubilised. Reverse micelle have a larger interface or surface area which has the ability to solubilise more penicillin $G$ molecules compared to the aqueous and organic phase interface. Moreover, with the mixing process, the distance between the penicillin G and AOT molecules is reduced and the attraction between these molecules is stronger. The solubilisation of penicillin $\mathrm{G}$ molecules in the reverse micelle was also improved by addition of salt because without salt the solution tends to form an emulsion and it was difficult to get clear phase separation (as discussed in Section 4.5.1).

$K_{f w}^{\infty}$ and $K_{b w}^{\infty}$ do not describe the same equilibrium due to the different physical system that occur during forward and backward extraction, which is why $K_{f w}^{\infty} \neq$ $1 / K_{b w}^{\infty}$. Moreover, the starting conditions between both extraction systems are also different. As discussed in Section 5.2.4, there is a layer of AOT molecules at the aqueous/organic interface. During the renewal process at the interface, it is possible that reorientation of the molecules contributes to rupture of the reverse micelle. At the interface, the AOT 'head' is in contact with bulk water and reverse micelle is 
attached to AOT at the interface. This will enable the water in the reverse micelle to have a contact with the bulk water and hence be released into the immiscible water phase. In backward extraction, despite the hydrophilic nature of penicillin $G$ molecules, the interaction between penicillin G, AOT and water is strong. Due to their hydrophilicity, entrapped penicillin $G$ molecules cannot be transported out of the reverse micelle structure. Since the in the water pool is similar to that in the bulk phase (Leodidis and Hatton, 1990; Rabie and Vera, 1996; Dokter et al., 2007), the penicillin $\mathrm{G}$ molecules are stable in the water pool, additionally the strong interaction between the molecules and AOT make them difficult to move out from the reverse micelle. The mixing process is important during backward extraction to promote contact between the reverse micelle and aqueous phase. This will possibly increase the interfacial areas for mass transfer by rupturing the reverse micelle, so that the penicillin $\mathrm{G}$ molecules can be transferred into the aqueous phase. However, although the reverse micelle are ruptured most of the penicillin $\mathrm{G}$ and AOT molecules are still attached together, but water in the water pool is transferred to the bulk water resulting in an increased volume of the backward aqueous phase (as discussed in Section 5.2.1). After the extraction process, the penicillin $G$ and AOT molecules move back to the organic phase, with some of the penicillin $\mathrm{G}$ molecules released from the reverse micelle staying in the aqueous phase.

\subsection{Suggestion for Improving the Backward Extraction}

The reverse micelle process developed for practical use needs to represent not only forward extraction with high efficiency, but also give a sufficient yield during backward extraction. It seems that the thermodynamically stable nature of reverse micelle make it difficult to release the penicillin $G$ molecules during backward extraction. Attempts were made in this research to enhance the backward extraction by manipulating important parameters such as salt concentration, surfactant concentration, $\mathrm{pH}$ and initial concentration of penicillin $\mathrm{G}$. However, the extraction of penicillin $\mathrm{G}$ in the backward process is found to be poor in respect of the overall efficiency (as shown in Figure 5.10), when taking the forward and backward process together, is consequently lower than might be expected. However, such a problem in apparent in most of the research on this topic, which suggests that further research on 
how to increase the extraction yield during the backward extraction process is required.

As discussed in Chapter 2, addition of alcohol as a co-surfactant in the reverse micelle solution can improve backward extraction in some circumstances. Table 5.3 shows comparison of the results found by other researches, both with and without alcohol addition. In the current study, the maximum percentage of penicillin $G$ solubilised in the backward aqueous phase is about 60\% (refer Figure 5.10).

Table 5.3: Efficiency of backward extraction as reported by several authors.

\begin{tabular}{|c|c|c|c|c|}
\hline Author & Surfactant & Biomolecule & $\begin{array}{c}\% \text { backward extraction } \\
- \text { no alcohol }\end{array}$ & $\begin{array}{c}\text { \% backward extraction } \\
\text { - with alcohol }\end{array}$ \\
\hline Mathew and Juang (2007) & AOT & papain & 30 & 90 \\
Juang et al. (2006) & AOT & lysozyme & no data & 90 \\
Liu et al. (2004) & AOT & nattokinase & no data & 80 \\
Shin et al (2003) & AOT & lysozyme & 0 & 100 \\
Yu et al. (2003) & AOT & lipase & 0 & 70 \\
Zhang et al. (2002) & CTAB & BSA & 0 & 90 \\
Goto et al. (1998) & AOT & chymotrypsin & 30 & 80 \\
Ono et al. (1996) & DOLPA & haemoglobin & 0 & 80 \\
Pires and Cabral (1993) & CTAB & cytochrome & 0 & 80 \\
Carlson and Nagarajan (1992) & AOT & bovine cymosin & no data & 70 \\
Carlson and Nagarajan (1992) & AOT & porcine pepsin & no data & $\sim 100$ \\
Aires-Barros and Cabral (1991) & AOT & lipase B & no data & 85 \\
\hline
\end{tabular}

As seen in Table 5.3, many of the results show that without alcohol addition, there is $0 \%$ of backward extraction. Findings from this study (60\%) give relatively good results when compared to others, especially as AOT is the surfactant. As discussed in Section 5.3.1.2, the difficulties of solubilising penicillin $G$ into the backward aqueous phase arise from the strong interaction between the hydrophilic part of the reverse micelle and the penicillin G molecules. Mathew and Juang (2007) recently stressed that the strong interaction between the solublised biomolecules and reverse micelle induces micelle-micelle interaction, which results in a decrease of back extraction. They suggested that the control of micelle-micelle interactions may be a very important factor for the success of backward extraction process. Moreover, 
Hong et al., (2000) suggested that the alcohol molecule could be a good modifying agent for reverse micelle. Therefore, it is important for future studies to take into account the addition of alcohol to increase the results in the backward extraction process. Careful consideration of the type of alcohol suitable for penicillin $G$ extraction and the interaction of the alcohol with the penicillin structure, has to be given.

\subsection{Conclusions}

Backward extraction of the penicillin $\mathrm{G}$ from an AOT-isooctane reverse micelle solution to a fresh aqueous solution containing $\mathrm{KCl}$ was studied to find favourable conditions for penicillin $\mathrm{G}$ recovery. The partitioning of penicillin $\mathrm{G}$ between the two phases was investigated for various values of salt concentration, $\mathrm{pH}$, surfactant concentration, initial penicillin $\mathrm{G}$ concentration in the forward extraction, and AOT concentration. A maximum mass of penicillin $G$ was found to solubilise from reverse micelle at $37 \mathrm{~g} / 1$ of $[\mathrm{KCl}]$ and $267 \mathrm{~g} / \mathrm{l}$ of surfactant concentration. $\mathrm{pH}$ shows a small effect on the backward extraction due to the low $\mathrm{pI}$ value of penicillin $\mathrm{G}$ which allows solublisation over a wide range of $\mathrm{pH}$. Despite relatively small masss of penicillin being transferred into the backward aqueous phase, all water that was transferred in the reverse micelle during forward extraction was transferred back in the aqueous phase during backward extraction.

In the backward extraction process, the dependency of the distribution coefficient on penicillin concentration, AOT concentration, and $\mathrm{pH}$ was evaluated using the Langmuir and Brandani models. From experimental results the distribution coefficient at infinite dilution during backward extraction $\left(K_{E D_{w}}^{\infty}\right)$ depends markedly on surfactant concentration and is fairly independent of $\mathrm{pH}$. The $K_{E \cdot b w}^{\infty}$ for both $\mathrm{pH}$ were similar except at high surfactant concentration. The $K_{E . b w}^{\infty}$ values were different for both the Langmuir and Brandani models, and neither fitted well to the experimental data for the backward extraction. 
The physical system is completely different between the forward and backward extraction processes. $K_{f w}^{\infty} \neq 1 / K_{b w}^{\infty}$ because of the two different physical systems. 


\section{CHAPTER SIX}

\section{Conclusion and Recommendations}

\subsection{Introduction}

This chapter concludes the thesis. It starts with the main conclusions from the research and also presents the limitations of the research and recommendations for further research.

\subsection{Overall Conclusions}

In this study, the solubilisation of penicillin $\mathrm{G}$ from aqueous phase consisting of $\mathrm{KCl}$ and water into an organic phase consisting AOT and isooctane was investigated. Several parameters were used to determine the optimum condition and the dependency of the extraction process, including surfactant concentration, $\mathrm{pH}$, salt concentration and salt type. Other properties such as critical micelle concentration (CMC), surface tension and water content were investigated to establish more understanding of the characteristics of reverse micelles. The analytical methods used were the Kjeldahl method for penicillin $G$ concentration determination, the Karl Fischer titration method for water content analysis and the Wilhelmy Ring method for surface tension and $\mathrm{CMC}$ analysis.

For forward extraction, the results show that penicillin is an interfacially active compound that interacts with AOT, with the interfacial association being dependent on both $\mathrm{pH}$ and surfactant concentration. The vertical displacement of the experimental data from the $45^{\circ}$ line is a measure of the strength of interfacial association. During experimental work, it has been found that when the concentration ratio $[P]_{a q} /[S]$ is high precipitation of the penicillin occurs. 
In the forward extraction, there is optimum surfactant concentration at $222 \mathrm{~g} / \mathrm{l}$ which corresponds to the maximum limit for penicillin $G$ transfer into the reverse micelles. A maximum penicillin $\mathrm{G}$ concentration in the reverse micellar phase at low $\mathrm{pH}$ values was also found. This could be attributed to the high electrostatic attraction between AOT anions and the positively charged penicillin $\mathrm{G}$ aggregates at $\mathrm{pH}<\mathrm{pI}$. The penicillin concentration in the organic phase was found to decrease when salt concentration increased above $10 \mathrm{~g} / \mathrm{l}$. Although high salt concentration decreased the solubilisation of penicillin $\mathrm{G}$ into reverse micelles, the addition of salt to the aqueous penicillin G solution was very important in order to avoid the formation of a stable emulsion. The solubilisation of penicillin $\mathrm{G}$ into the reverse micelles is higher when using a divalent salt such as $\mathrm{CaCl}_{2}$ due to the ability of smaller reverse micelles to solubilise more penicillin. However, the disadvantage of using $\mathrm{CaCl}_{2}$ was that the solution became more sensitive to the stirring speed and tended to emulsify more easily. The water content in the system without penicillin was found to be higher than that with penicillin and the calculated reverse micelles radii were around $3 \mathrm{~nm}$.

The Langmuir and Brandani models were used for predictions of penicillin G partitioning in the aqueous phase and reverse micelle solution. Using the concentration of penicillin $\mathrm{G}$ in both the aqueous and reverse micelle organic phases, the distribution coefficient $(K)$ was calculated. The predictions were in very good agreement with the experimental data. The distribution coefficient favours transfer of the penicillin into the reverse micelles at moderate AOT concentrations. The distribution coefficient at infinite dilution, $K_{f}^{\infty}$, was shown to be a function of both $\mathrm{pH}$ and surfactant concentration; similar trends in the value of $K_{f}^{\infty}$ were observed at different $\mathrm{pH}$ values and $K_{f}^{\infty}$ decreased as the surfactant concentration was increased. Compared to the Langmuir model, the Brandani model was found to more relevant to the current work. The Brandani model is more representation of a real physical system because it describes the thermodynamic distribution dispersion, not only at the interfacial phase (as only describe by Langmuir model), but in the reverse micelle phase as well.

Despite successful results in the forward extraction, the extraction of penicillin $G$ in the backward process was poor. The overall yield, when taking the forward and backward process together, was consequently lower than might be expected - that is, 
the penicillin recovered from the backward process was less than the initial mass of penicillin $\mathrm{G}$ present in the aqueous phase before forward extraction $\left(P_{\text {aq.i.fw }}\right)$. It has been found that the mass of water transferred into the reverse micelles during forward extraction was transferred back into the aqueous phase during backward extraction resulted increasing volume of the backward aqueous phase. However, the additional volume of the backward aqueous phase does not correspond to the mass of penicillin $\mathrm{G}$ solubilised during backward extraction. Salt concentration plays an important role during backward extraction. By increasing its value, the mass of penicillin $\mathrm{G}$ in the aqueous phase $\left(P_{\text {aq.f.bw }}\right)$ increased significantly. The $P_{\text {aq.f.bw }}$ reached at optimum at a $\mathrm{KCl}$ concentration of $37 \mathrm{~g} /$.

Contrary to the forward extraction, the values of $K_{b w}^{\infty}$ are different to both Langmuir and Brandani models for the backward extraction which shows that the models do not fit very well to the experimental data. $K_{f w}^{\infty} \neq 1 / K_{b w}^{\infty}$ because the interaction between penicillin $G$ and reverse micelles is very strong, despite the hydrophilic nature of the penicillin $\mathrm{G}$ molecules. Entrapped penicillin $\mathrm{G}$ molecules are difficult to extract from the reverse micelle structure despite the mixing process promoting contact between the reverse micelles and aqueous phase. Since the poor yield in the backward extraction has also been reported by most other researchers in reverse micelle extraction, a potential solution to this problem may be the addition of alcohol during the backward extraction.

\subsection{Constraints of the Study}

All research studies have their constraints, and this study is no exception. The main limitations were as follows:

- There is no work published so far on the thermodynamic framework of solubilisation of antibiotics, and there is a limited mass of thermodynamic modelling of protein solubilisation. This makes it difficult to compare the results from this study with other data. For the purpose of experimental and 
modelling work, the parameters used are based on the reverse micelle extraction of protein, although the system is completely different to this study.

- The reverse micelle extraction process of penicillin $\mathrm{G}$ did not provide a real system as the penicillin $G$ solution is not from a fermentation broth. However, by using the real system selectivity of the extraction is expected to be different compared to current study due to presence of impurities in the broth.

\subsection{Recommendations for Future Work}

Based on the findings of this study, it is recommended that further work be carried out to investigate other factors which may improve the backward extraction process. The addition of alcohol is obviously the most important parameter due to its successful application in the backward extraction of protein by other researchers. A substantial mass of work regarding alcohol addition has to be considered because different alcohols could be expected to react differently with the type of surfactant and biomolecules used. Therefore, by using the right alcohol as a co-surfactant, the strong interaction between reverse micelles and penicillin $\mathrm{G}$ could be reduced to provide an increase of the overall extraction yield.

The better understanding of reverse micelle structure and properties is an advantage so that better explanations are available for the problems that can occur during the solubilisation process, such as precipitation and low extraction/backward extraction yield. Moreover a better understanding of the hydrophilic and hydrophobic behaviour of reverse micelles could be used to increase partitioning selectivity of biomolecules.

It is recommended that further experiments are conducted using a 'real' system, i.e., a penicillin $\mathrm{G}$ solution obtained from a fermentation broth. The experimental work is expected to be more difficult due to a number of impurities in the fermentation broth solution and lack of available literature. It is evident that most studies of reverse 
micelle extraction to date have used biomolecules in pure systems not in real systems.

Experiments could be carried out using prototype equipment, which is representative of real equipment that could be used in industry. One of the advantages of reverse micelles system is the surfactant solution can be separated from the whole solution by filtration and the organic phase containing surfactant recycled. It is recommended that the prototype equipment is designed to operate in a continuous manner so that the recycled surfactant can be used for many times. 


\section{References}

Adachi M., Harada M. and Katoh S., 2000, Bioaffinity separation of chymotrypsinogen using antigen-antibody reaction in reverse micellar system composed of a non-ionic surfactant, Biochem. Eng. J., 4: 149-151.

Aires-Barros M.R. and Cabral J.M.S., 1991, Selective separation and purification of two lipases from chromobacterium viscosum using AOT reversed micelles, Biotech. Bioeng., 38:1302-1307.

Andrews B.A., 1993, The effect of protein characteristics on their extraction in reversed micelle systems, Sep. Biotech., 3: 29-36.

Andrews B.A. and Haywood K., 1994, Effect of pH, ion type and ionic strength on peartitioning of protein in reversed micelle system, J. Chromat. A., 668: 55-60.

Ashrafizadeh S.N. and Khoshkbarchi M.K., 1998, Modelling and experimental data for the reverse micellar extraction of proteins using a new surfactant, Sep. Sci. Technol., 33(16): 2579-2595.

Baier G., 1999, Liquid-Liquid Extraction Based on a New Flow : Two Fluid TaylorCouette Flow. University of Wisconsin-Madison, Ph.D. Thesis.

Bardez E., Giordano R., Janneli M.P., Migliardo P. and Wanderlingh U., 1996, Hydrogen-bond effects induced by alcohol on the structure and dynamics of ionic reverse micelles, J. Mol. Struct., 383: 183-190.

Bong K.L, Dong P.H, Sung S.L and Kuboi R., 2004, Analysis of protein backextraction processes in alcohol-and carboxylic acid-mediated AOT reverse micellar systems based on structural changes of proteins and reverse micelles, Biochem. Eng. J., 22: 71-79. 
Brandani S., Brandani V., Giacomo G.D., 1994, A thermodynamic model for protein partitioning in reverse micellar systems, Chem. Eng. Sci., 49(21): 3681-3686.

Brandani V., Giacomo G.D. and Spera L., 1996, Recovery of a-amylase extracted by reverse micelles, Proc. Biochem., 31(2): 125-128.

Bruno P., Caselli M., Luisi P.L., Maestro M., Traini A., 1990, A simplified thermodynamic model for protein uptake by reverse micelles: theoretical and experimental results, J. Phys. Chem., 94(15): 5908-5917.

Cardoso M. M., Barradas M. J., Kroner K. H. and Crespo J. G., 1999, Amino acid solubilization in cationic reversed micelles: factors affecting amino acid and water transfer, J. Chem. Technol. Biotechnol., 74: 801-811.

Carlson A. and Nagarajan R., 1992, Release and recovery of porcine pepsin and bovine cymosin from reverse micelles: A new technique based on isopropyl alcohol addition, Biotechnol. Prog., 8: 85-90.

Carvalho C.M.L. and Cabral J.M.S., 2000, Reverse micelles as reaction media for lipases, Biochimie, 82: 1063-1085.

Cascaval D., Oniscu C., Dumitru I.F. and Galaction A., 2001, New extraction techniques in biotechnology, Roum. Biotechnol. Lett., 6(3): 207-232.

Castagnola M.J. and Dutta P.K., 2000, Synthesis of microporous faujasitic-like zincophosphates from reverse micelles, Microporous and Mesoporous Mat., 34: 6165.

Chang Q.L., Chen J.Y., Zhang X.F. and Zhao N.M., 1997, Effect of the cosolvent type on the extraction of $\alpha$-amylase with reversed micelles: circular dichroism study, Enz. Microb. Technol., 20: 87-92.

Chang G.G., Huang T.M. and Hung H.C., 2000, Reverse micelles as a lifemimicking system, Proc. Nat. Sci. Counc. ROC (B), 24(3): 89-100. 
Cheng H. and Sabatini D.A., 2001, Reverse-micellar extraction for micellarsolubilized contaminant and surfactant removal, Sep. Purif. Tech., 24: 437-449.

Chimuka L., Cukrowska E. and Jonsson A., 2004, Why liquid membrane extraction is an attractive alternative in sample preparation, Pure Appl. Chem., 76(4): 707-722.

Debnath S., Das D. and Das P.K., 2007, Unsaturation at the surfactant head: Influence on the activity of lipase and horseradish peroxidise in reverse micelles, Biochem. Biophys. Res. Com., 356: 163-168.

Dekker M., Van't Riet K., Bijsterbosch B.H., Wolbert R.B.G. and Hilhorst R., 1989, Modelling and optimization of the reversed micellar extraction of a-amylase, AIChE $J .$, 35: 315- 321 .

Dekker M., Van't Riet K., Bijsterbosch B.H., Fijneman P. and Hilhorst R., 1990, Mass transfer rate of protein extraction with reversed micelles, Chem. Eng. Sci., 45: 2949-2957.

Dokter A.M., Woutersen S. and Bakker H.J., 2006, Inhomogeneous dynamics in confined water nanodroplets, Proc. Natl. Acad. Sci. New York. 103:15355-15358.

Dokter A.M., Woutersen S. and Bakker H.J., 2007, Ultrafast dynamics of water in cationic micelles, J. Chem. Phys., 126:124-507.

Dövyap Z., Bayraktar E. and Mehmetoğlu V., 2006, Amino acid extraction and mass transfer rate in the reverse micelle system, Enz. Microb. Tech., 38: 557-562.

Dungan S.R., Bausch T., Hatton T.A., Plucinski O. and Nitsch W., 1991, Interfacial transport processes in the reversed micellar extraction of proteins, J. Colloid Interf. Sci., 145: 33-50.

Elander R.P., 2003, Industrial production of $\beta$-lactam antibiotics, Appl. Microbiol. Biotechnol., 61: 385-392. 
Esalah, J., 1997, Removal of heavy metals from aqueous solutions by precipitation with sodium di-(n-octyl)phosphinate. McGill University, Canada: Ph.D. Thesis.

Feng K.I., 1995, Equilibrium properties and dynamic behavior of crystallites and reverse micelles of sodium bis (2-ethylhexyl) phosphate in benzene. University of Texas: Ph.D. Thesis.

Franqueville E., Loutrari H, Mellou F., Stamatis H., Friboulet A. and Kolisis F.N., 2003, Reverse micelles, a system for antibody-catalysed reaction, J. Mol. Cata. B, 21(1-2): 15-17.

Freda M., Onori G., Paciaroni A. and Santucci A., 2002, Influence of hydration on dynamical properties of reverse micelles, J. Non-Crystalline Solids., 30: 874-877.

Frense D., Hadtendorn R. and Ulbrich-Hofmann R., 1995, 2-Modified 1,3diacylglycerols as new surfactants for the formation of reverse micelles, Chem. Phys. Lipids., 78: 81-87.

Furusaki S. and Kishi K., 1992, Extraction of amino acids and peptides by reverse micelles in Solvent Extraction, Elsevier Science, New York.

Gerson D.F., 1988, Liquid-liquid separation in biotechnology in BiotechnologyResearch and Application, Elsevier, New York.

Ghosh A.C., Mathur R.K. and Dutta N.N., 1997, Extraction and purification of cephalosporin antibiotics in Advances in Biochemical Engineering/Biotechnology. Vol. 56, Springer-Verlag, Berlin Heidelberg.

Goklen K. and Hatton T.A., 1987, Liquid-liquid extraction of low molecular weight proteins by selective solubilization in reverse micelles, Sep. Sci. Technol., 22: 831841. 
Goto M., Ishikawa Y., Ono T., Nakashio F. and Hatton T.A., 1998, Extraction and activity of chymotrypsin using AOT-DOLPA mixed reversed micellar systems, Biotechnol. Prog., 14: 729-734.

Goto M., Ono T., Horiuchi A. and Furusaki S., 1999, Extraction of DNA by reversed micelles, J. Chem. Eng. Japan, 32(1): 123-125.

Goto M., 2006, Recent advance in protein extraction and chiral separation of biomolecules, Tsinghua Sci. Tech., 11(2): 194-201.

Grinbaum B., 2003, An integrated method for development and scaling up of extraction process in Ion Exchange and Solvent Extraction. Vol.15. Marcel Dekker, New York.

Gu T. Y., 2000, Liquid-liquid partitioning methods for bioseparations in Handbook of Bioseparations, pp 329-364, Academic Press, New York.

Haghtalab A. and Osfouri S., 2003, Vacancy solution theory for partitioning of protein in reverse-micellar system, Sep. Sci. Tech., 38(3): 553-569.

Hano T., Michiaki M. and Takaaki O., 1994, Continuous extraction of penicillin G with liquid surfactant membrane using Vibro Mixer ${ }^{\circledR}$, J. Membrane Sci., 93: 61-68.

Hasmann F.A., Cortez D.V., Junior A.P. and Roberto I.C., 2003, Optimization of $\beta$ xylosidase recovery by reversed micelles using response surface methodology, Elect. J. Biotech., 6(2): 3445-3458.

Hasmann F.A., Cortez D.V., Gurpilhares D.B., Santos V.C., Roberto I.C. and Junior A.P., 2007, Continuous counter-current purification of glucose-6-phosphate dehydrogenase using liquid-liquid extraction by reverse micelles, Biochem. Eng. J., 34: $236-241$.

Hatton T.A., 1985, Liquid-liquid extraction of proteins in Comprehensive Biotechnology (2)., Pergamon Press, London. 
Havre T.E., 2002, Formation of calcium naphthenate in water/oil systems, naphthenic acid chemistry and emulsion stability. Norwegian University of Science and Technology Trondheim: Ph. D. Thesis.

Hecht H.G. and Peled H.B., 2006, Structure modifications of AOT reverse micelles due to protein incorporation, J. Coll. Interface Sci., 297: 276-283.

Ho W.S.W. and Li N.N., 1992, Emulsion liquid membrane in Membrane Handbook, van Nonstrand Reinhold, New York.

Hong D.P and Kuboi R., 1999, Evaluation of the alcohol-mediated interaction between micelles using percolation processes of reverse micellar systems, Biochem. Eng. J., 4: 23-29.

Hong D.P, Lee S.S. and Kuboi R., 2000, Conformational transition and mass transfer in extraction of proteins by AOT-alcohol-isooctane reverse micellar systems, $J$. Chromatoghrapy B, 743: 203-213.

Hossain M.J., Takeyama T., Hayashi Y., Kawanishi T., Shimizu N. and Nakamura R., 1999, Enzymatic activity of Chromobacterium viscosum lipase in an AOT/Tween 85 mixed reverse micellar system, J. Chem. Technol. Biotechnol., 74: 423-428.

Hu Z. and Gulari E., 1996, Protein extraction using the sodium bis (2-ethylhexhyl) phosphate (NaDEHP) reverse micellar system, Biotechnol. Bioeng., 50: 203-206.

Ichikawa S., Imai M. and Shimizu M., 1992, Solubilizing water involved in protein extraction using reversed micelles, Biotechnol. Bioeng., 39: 20-26.

Imai M., Natsume T., Naoe K., Shimizu M., Ichikawa S. and Furusaki S., 1996, Hydrophilic surroundings requisite for the solubilization of proteins related with their hydrophobicity in the AOT reversed micellar extraction, Biosep., 6: 325-333. 
Ismael M. and Tondre C., 1992, Kinetically controlled separation of nickel (11) and cobalt (11) using micelle-solubilised extractant in membrane processes, Langmuir, 8: 1039-1041.

Jolivalt C., Minier M. and Renon H., 1993, Extraction of cytochrome $c$ in sodium dodecylbenzenesulfonate microemulsions, Biotechnol. Prog., 9: 456-461.

Juang R.S., Kao H.C. and Shiau C.L., 2006, Kinetic analysis on membrane-based reverse micellar extraction of lysozyme from aqueous solutions, J. Memb. Sci., 281: 636-645.

Jun G. L., Jian M.X., Rui S., Cheng L.Y. and Hui Z.L., 2004, Reverse micelles extraction of nattokinase from fermentation broth, Biochem. Eng. J., 21: 273-278.

Kamiya N., Goto M. and Nakashio F., 1995, Surfactant-coated lipase suitable for the enzymatic resolution of methanol as a biocatalyst in organic media, Biotech. Prog., 11: $270-275$.

Kilikian B.V., Bastazin M.R., Minami N.M., Goncalves M.R. and Junior A.P., 2000, Liquid-liquid extraction by reversed micelles in biotechnological processes, Braz. $J$. Chem. Eng., 17: 29-38.

Kinugasa T., Tanahashi S. I and Takeuchi H., 1991, Extraction of lysozyme using reverse micellar solution: Distribution and extraction rates, Ind. Eng. Chem. Res., 30: 2470-2476.

Kinugasa T., Kondo A., Mouri E., Ichikawa S., Nakagawa S., Nishii Y., Watanabe K. and Takeuchi H., 2003, Effects of ion species in aqueous phase on protein extraction into reversed micellar solution, Sep. Purif. Tech., 31: 251-259. 
Kishi K. and Furusaki S., 1992, Effect of hydrophobicity on reverse micellar extraction of oligopeptides, J. Chem. Eng. Japan, 23(1): 91-93.

Krieger N., Taipa M.A.,Aires-Barros M.R., Melo E.H.M., Lima-Filhoc J.L. and Cabral J.M.S., 1997, Purification of the citrinum Lipase Penicillium using reversed micelles AOT, J. Chem. Tech. Biotechnol., 69: 77-85.

Kyung H.N. and Jee Y.I., 2005, One-step separation of lysozyme by reverse micelles formed by the cationic surfactant, cetyldimethylammonium bromide, Food Chem., 93(1): 95-101.

Lee C.K. and Sandler S.I., 1990, Vancomycin partitioning in aqueous two-phase system: effects of ph, salts, and an affinity ligand, Biotech. Bioeng., 35: 408-416.

Lee S.S., Lee B.K., Choi J.S. and Lee J.P., 2001, Effect of alcohol addition on backextraction of BSA and cytochrome $\mathrm{C}$ using AOT reverse micellar system, Bull. Korean Chem. Soc., 22(8): 897-902.

Leodidis E.B. and Hatton T.A., 1989, Specific ion effects in electrical double layers: Selective solubilization of cations in Aerosol-OT reversed micelles, Langmuir, 5: 741-753.

Leodidis E.B. and Hatton T.A., 1990, Amino acids in AOT reversed micelles. 1. Determination of interfacial partition coefficient using the phase-transfer method, $J$. Phys. Chem., 94: 6400-6411.

Leser, M.E and Luisi P.L., 1990, Application of reverse micelles for the extraction of amino acids and protein, Chimia, 44: 270-280.

Liu J., Wang W. and Li G., 2001, A new strategy for supercritical fluid extraction of copper ions, Talanta, 53: 1149-1154.

Liu J.G., Xing J.M., Shen R., Yang C.L. and Liu H.Z., 2004, Reverse micelle extraction of nattokinase from fermentation broth, Biochem. Eng. J., 21: 273-278. 
Liu Y., Dong X.Y. and Sun Y., 2006, Equilibria and kinetics of protein transfer to and from affinity-based reverse micelles of Span 85 modified with Cibacron Blue F3GA, Biochem. Eng. J., 28: 281-288.

Lye J.L., 1993, Kinetic studies on the extraction of proteins using reverse micelles. University of Reading, PhD Thesis,UK.

Lye G.J., Asenjo J.A and Pyle D.L., 1995, Extraction of lysozyme and ribonucleasea using reversed micelles: Limits to protein solubilization, Biotechnol. Bioeng., 47(5): 509-519.

Majumdar T. and Mahapatra A., 2007, Kinetics of electron transfer reaction in micellar and reverse micellar media - Reduction of $\left[\mathrm{Co}\left(\mathrm{NH}_{3}\right)_{5} \mathrm{~N}_{3}\right] \mathrm{Cl}_{2}$ by iron (II), Coll. Surf. A, 302: 360-365.

Mat H.B. and Stuckey D.C., 1994, Solvent selection criteria for protein extraction using reverse micellar systems, Sep. Biotech., 3: 301-307.

Mathew D. S. and Juang R. S., 2007, Improved back extraction of papain from AOT reverse micelles using alcohols and a counter-ionic surfactant, Biochem. Eng. J., 25: 219-225.

Mathew D.S. and Juang R.S., 2007, Role of alcohols in the formation of inverse microemulsions and back extraction of protein/enzymes in a reverse micellar system, Sep. Purif. Tech., 53: 199-215.

Michaels M.A., Sherwood S., Kidwell M., Allsbrook M.J., Morrison S.A., Rutan S.C. and Carpenter E.E., 2007, Quantitative model for prediction of hydrodynamic size of non-ionic reverse micelles, J. Coll. Interface. Sci., 311: 70-76.

Moore S.A. and Palepu R.M., 2007, Fluorometric investigations on the transition from reverse micelles to microemulsions in non-aqueous microemulsions, $J . \mathrm{Mol}$. Liq., 135: 123-127. 
Moreno-Hagelsieb G., Gomez-Puyou A. and Soberon X., 1999, Eschecichia coli TEM $1 \beta$-lactamase in CTAB reverse micelles: Exchange/diffusion-limited catalysis. FEBS Letters, 459: 111-114.

Nabais A.M.A and Cardoso J.P., 1999, Purification of benzylpenicillin filtered broths by ultrafiltration and effect on solvent extraction, Bioproc. Biosyst. Eng., 21: 157163.

Nagayama K., Nishimura R., Doi T. and Imai M., 1999, Enhanced recovery and catalytic activity of Rhizopus delemar lipase in an AOT microemulsion system with guanidine hydrochloride, J. Chem. Technol. Biotechnol., 74: 227-230.

Nakashio F., Goto M. and Kondo K., 1992, New surfactants for metal extraction by liquid membrane in Solvent Extraction, Elsevier, New York.

Naoe K., Nishino M., Ohsa T., Kawagoe M. and Imai M., 1999, Protein extraction using sugar ester reverse micelles, J. Chem. Technol. Biotechnol., 74: 221-226.

Naoe K., Kai T., Kawagoea M. and Imai M., 1999, Extraction of flexibly structured protein in AOT reverse micelles: the flexible structure of protein is the dominant factor for its incorporation into reverse micelles, Biochem. Eng. J., 3: 79-85.

Naoe K., Murata M., Ono C., Kawagoe M. and Imai M., 2002, Efficacy of guanidium salts in protein recovery from reverse micellar organic media, Biochem. Eng. J., 10: 137-142.

Naoe K., Noda K., Kawagoe M. and Imai M., 2004, Higher order structure of proteins solubilized in AOT reverse micelles, Coll. Surf. B., 38: 179-185.

Nishiki T., Sato I., Katoaka T. and Kato D., 1993, Partitioning behaviour and enrichment of proteins with reversed micellar extraction: 1. Forward extraction of proteins from aqueous to reversed micelle phase, Biotechnol. Bioeng., 42: 596-600. 
Nishiki T., Muto A., Kataoka T. and Kato D., 1995, Back extraction of proteins from reversed micellar to aqueous phase: partitioning behaviour and enrichment, Biochem. Eng. J., 59: 297-301.

Nishiki T., Sato I., Muto A. and Kataoka T., 1998, Mass transfer characterization in forward and back extractions of lysozyme by AOT-isooctane reverse micelles across a flat liquid-liquid interface, Biochem. Eng. J., l: 91-97.

Noble M.J. and Varley J., 1990, Colloidal gas asprons generated from the anionic surfactant AOT for the separation of proteins from aqueous solution, J. Chem. Technol. Biotechnol., 74: 231-237.

Noh K.H. and Imm J.Y., 2005, One-step separation of lysozyme by reverse micelles formed by the cationic surfactant, cetyldimethylammonium bromide, Food Chem., 93: 95-101.

Ono T., Goto M., Nakashio F. and Hatton T.A., 1996, Extraction behaviour of haemoglobin using reversed micelles by diolyel phosphoric acid, Biotechnol. Prog., 12: $793-800$.

Pandit P and Basu S., 2002, Removal of organic dyes from water by liquid-liquid extraction using reverse micelles, J. Coll. Interface Sci., 245: 208-214.

Paradkar V.M. and Dordick S., 1994, Affinity-based reverse micellar extraction and separation (ARMES): A facile technique for the purification of peroxidase from soybean hulls, Biotech. Prog., 9: 199-203.

Paul B.K. and Mitra R.K., 2006, Percolation phenomenon in mixed reverse micelles: the effect of additives, J. Coll. Interface Sci., 295: 230-242.

Pellegrino J.J. and Noble R.D., 1990, Enhance transport and liquid membranes in bioseparations, Tibtech., 8: 216-224. 
Pessoa J. A. and Vitolo M., 1998, Recovery of inulinase using BDBAC reversed micelles, Proc. Biochem., 33(3): 291-297.

Perez-Casas S., Castillo R. and Costas M., 1997, Effect of alcohols in AOT reverse micelles - a heat capacity and light scattering study, J. Phys. Chem. B., 101: 70437054.

Pileni M.P., 2006, Reverse micelles used as templates: A new understanding in nanocrystal growt, J. Exp. Nanosci., 1(1): 13-27.

Pires M.J. and Cabral J.M.S., 1993, Liquid-liquid extraction of a recombinant protein with a reverse micelle phase, Biotechnol. Prog., 9: 647-650.

Pires M.J., Aires-Barros M.R. and Cabral J.M.S., 1996, Liquid-liquid extraction of proteins with reverse micelles, Biotechnol. Prog., 12: 290-301.

Rabie H.R. and Vera J.H., 1996, Extraction of zwitterionic amino acids with reverse micelles in the presence of different ions, Ind. Eng. Chem. Res., 35: 3665-3672.

Rabie H.R., Helou D., Weber M.E. and Vera J.H., 1997, Comparison of the titration and contacts methods for the water solubilization capacity of AOT reverse micelles in the presence of a cosurfactant, J. Coll. Interface Sci., 189: 208-215.

Rabie H.R., Vera J.H., 1998, A simple model for reverse micellar extraction of proteins. Sep. Sci. Technol. 33(8): 1181-1193.

Regalado C., Asenjo J.A and Pyle D.L., 1996, Studies on the purification of peroxidase from horseradish roots using reverse micelles, Enz. Microb. Tech., 16: 332-339.

Rito-Palomares M., Huddleston J.G. and Lyddiatt A., 1996, Phase recycling in aqueous two-phase partition processes: impact upon practical implementation of protein recovery from brewery waste, Sep. Biotech., 3: 413-418. 
Rodrigues E.M.G., Milagres A.M.F. and Jr A.P., 1999, Xylanase recovery: Effect of extraction conditions on the AOT-reversed micellar systems using experimental design, Proc. Biochem., 34: 121-125.

Shen C. W. and Yu T., 2007, Protein separation and entichment by counter-current chromatography using reverse micelle solvent systems, $J$. Chromatography A, 1151: 164-168.

Shin Y., Weber M.E. and Vera J.H., 2003, Comparison of two methods to recover lysozyme from reverse micellar phases, Sep. Sci. Tech., 38(8): 1733-1748.

Soto A., Arce A. and Khoshkbarchi M. K., 2005, Partitioning of antibiotics in a twoliquid phase system formed by water and a room temperature ionic liquid, Sep. Purif. Tech., 44: 242-246.

Spirovska G. and Chaudhuri J.B., 1998, Sucrose enhances the recovery and activity of ribonuclease A during reversed micelle extraction, Biotechnol. Bioeng., 58(4): 374-379.

Su W.D. and Lee C.K., 1999, Reversed micellar extraction of vancomycin: Effect of ph, salt concentration and affinity ligands, Sep. Sci. Tech., 34(8): 1703-1715.

Tessier L., Bouchard P. and Rahni M., 2005, Separation and purification of benzylpenicillin produced by fermentation using coupled ultrafiltration and nanofiltration technologies, J. Biotech., 116: 79-89.

Thien M.P., Hatton T.A. and Wang D.I.C., 1986, Separation and concentration of amino acids using liquid emulsion membranes, Biotech. Bioeng., 32: 604-615.

Tzeng Y.M., Tsun H.Y and Chang Y.N., 1999, Recovery of thuringiensin with cetylpyridinium chloride using micellar-enhanced ultrafiltration process, Biotechnol. Prog., 15: 580-586. 
Verral M. S., 1988, Solvent extraction in the pharmaceutical industry, Chem. Ind., 17: $648-656$.

Vyve F.V. and Renken A., 1999, Hydroformylation in reverse micellar systems, Cata. Today, 48: 237-243.

Wang W., Weber M.E. and Vera J.H., 1994, Effect of alcohol and salt on water uptake of reverse micelles formed by dioctyldimethyl ammonium chloride (DODMAC) in isooctane, J. Coll. Interface Sci., 168: 422-427.

Wang W., Weber M.E. and Vera J.H., 1995, Reverse micellar extraction of amino acids using dioctydimethylammonium chloride, Ind. Eng. Chem. Res., 34: 599-606.

Wang S., Zhou L., He W. and Hu Z., 2007, Separation and determination of alpinetin and cardamonin by reverse micelle electrokinetic capillary chromatography, $J$. Pharm. Biomedic. Ana., 43: 1557-1561.

Wolbert R.B.G., Hilhorst R., Voskuilen G., Nachtegaal H., Dekker M., van'tRiet K. and Bijterbosc B.H., 1989, Protein transfer from an aqueous phase into reversed micelles, European J. Biochem., 184: 627-633.

Woll J.M., Hatton T.A. and Yarmush M.L., 1989, Bioaffinity separations using reversed micellar extraction, Biotechnol. Prog., 5: 57-62.

www.rousselet-robatel.com

Xun F., Junling L., Ying M., Li Z., Debou W. and Zhengshui H., 2001, Amino acid extraction with AOT reverse micelle, Coll. Surf. A, 179: 1-10.

Yang W.Y., Lin C.D., Chu I.M and Lee C.J., 1994, Extraction of cephalosporin C from whole broth and separation of desacetyl cephalosporin $\mathrm{C}$ by aqueous two-phase partition, Biotechnol. Bioeng., 43: 439-445. 
Yang C. and Cussler E.L., 2000, Reactive extraction of penicillin G in hollow-fiber and hollow-fiber fabric modules, Biotechnol. Bioeng., 691: 66-73.

Yang X., 2001, Synthesis and Use of Chiral Surfactants. East Tennessee State University: Master Degree Thesis.

Yonker C.R., Fulton J.L., Phelps M.R. and Bowman L.E., 2003, Membrane separations using reverse micelles in nearcritical and supercritical fluid solvents, $J$. Supercritical Fluids, 25: 25-231.

Yu C.Y., Chu Y. and Ji J.Y., 2003, Study of the factors affecting the forward and back extraction of yeast-lipase and its activity by reverse micelles, $J$. of Colloid and Interf. Sci., 267: 1167-1174.

Yu C. Y., Chu Y. and Ji J. Y., 2003, Study of the factors affecting the forward and back extraction of yeast-lipase and its activity by reverse micelles, J. Coll. Interf. Sci., 267: 60-64.

Zhang T., Liu H. and Chen J., 1999, Affinity extraction of BSA by mixed reversed micellar system with unbound triazine dye, Biochem. Eng. J., 4: 17-21.

Zhang W., Liu H. and Chen J., 2002, Forward and backward extraction of BSA using mixed reverse micellar system of CTAB and alkyl halides, Biochem. Eng. J., 12: 15.

Zhang W., Qiao X., Chen J. and Wang H., 2006, Preparation of silver nanoparticles in water-in-oil AOT reverse micelles, J. Coll Interf. Sci., 302: 370-373.

Zhao J., Deng S., Liu J. and Zheng O., 2007, Fourier transform infrared investigation on the state of water in reverse micelles of quaternary ammonium Gemini surfactants $\mathrm{C}_{12}-s-\mathrm{C}_{12} .2 \mathrm{Br}$ in $n$-heptane, J. Coll. Interf. Sci., 311: 237-242. 
Zulauf M. and Eicke H.F., 1970, Inverted micelles and microemulsions in the ternary system $\mathrm{H}_{2} \mathrm{O}$ /Aerosol-OT/isooctane as studied by photon correlation spectroscopy, $J$. Phys Chem., 83: 480-486. 


\section{APPENDICES}

Appendix 1 Sample Calculation of the Final Concentration/Mass of Penicillin $\mathrm{G}$ in the Aqueous Phase, $[P]_{a q . f f w}$ and the Organic phase, $[P]_{r m+o . f . f w}$

Appendix 2 Experimental Results for Forward Extraction

Appendix 3 Experimental Results for Forward Extraction

Appendix 4 Thermodynamic Framework for Forward Extraction

Appendix 5 Thermodynamic Framework for Backward Extraction 


\section{Appendix 1}

Sample Calculation of the Final Concentration/Mass of Penicillin G in the Aqueous Phase, $[P]_{a q . f . f w}$, and the Organic Phase, $[P]_{m+o f f f w}$ 
The final concentration of penicillin $G$ in reverse micelles during forward extraction, $[P]_{r m+o f}$ is calculated using equations (3.3.)-(3.13) shown in Section 3.6.2.

\section{Parameters:}

Density of water, $\rho_{w}=1.0 \mathrm{~g} / \mathrm{ml}$

Volume of titration for blank sample, $V_{\text {titrantblank }}=0.6 \mathrm{ml}$

Volume of titration for sample, $V_{\text {titrant }}=3.0 \mathrm{ml}$

Molarity of acid, $M_{\text {acid }}=0.05 \mathrm{~mol} / 1$

Final volume of water in the aqueous phase, $V_{a q . f}=4.95 \mathrm{ml}$

Initial volume of water in aqueous phase, $V_{a q . i}=5.0 \mathrm{ml}$

Surfactant concentration, $[S]=88 \mathrm{mg} / \mathrm{ml}$

Initial concentration of penicillin $\mathrm{G}$ in the aqueous phase, $[P]_{\text {aq. } i}=1.8 \mathrm{mg}$ of penicillin/ml of water

Final concentration of penicillin $G$ in the aqueous phase, $[P]_{\text {aq. }}=0.34 \mathrm{mg} / \mathrm{ml}$

Final volume of water in the reverse micelles, $V_{r m+o . f}=V_{w . t}=0.05 \mathrm{ml}$

The percentage of nitrogen in the sample, $\% N$ as shown in equation (3.3);

$$
\begin{aligned}
& \% N=\frac{14.01\left(V_{\text {titrant }}-V_{\text {titrant.blank }}\right) M_{\text {acid }}}{10\left(\rho_{w} V_{\text {aq.f }}\right)}= \\
& \frac{\left[\left(14.01 \frac{\mathrm{g}}{\mathrm{mol}}\right)(3 \mathrm{ml}-0.6 \mathrm{ml})\left(0.05 \frac{\mathrm{mol}}{\mathrm{l}}\right)\right]}{\left[\left(1 \frac{\mathrm{g}}{\mathrm{ml}}\right)(4.95 \mathrm{ml}) 10\right]} \\
& =0.034
\end{aligned}
$$

Therefore, $\% N$ value is used to calculate the final amount of penicillin $G$ in the aqueous phase, $P_{a q . f}$;

$$
\begin{aligned}
P_{a q . f} & =\% N \rho_{w} V_{a q . f}\left(\frac{1}{100}\right) 1000=0.034\left(1 \frac{\mathrm{g}}{\mathrm{ml}}\right)(4.95 \mathrm{ml}) 10 \\
& =1.68 \mathrm{mg}
\end{aligned}
$$

The final concentration of penicillin $G$ in the aqueous phase, $[P]_{a q f}$, 


$$
[P]_{\text {aq. } f}=\frac{P_{a q . f}}{V_{a q . f}}=\frac{1.68 \mathrm{mg}}{4.95 \mathrm{ml}}
$$

$$
=0.34 \mathrm{mg} / \mathrm{ml}
$$

From the mass balance equation, the amount of penicillin $\mathrm{G}$ attached to the surfactant, $P_{s . f}$, is equal to $S[P]_{s . f}$ :

$$
\begin{aligned}
S[P]_{s . f} & =V_{a q . i}[P]_{a q . i}-V_{r m+o . f}[P]_{a q . i}-V_{a q . f}[P]_{a q . f} \\
S[P]_{s . f} & =P_{s . f}=(5 \mathrm{ml})\left(1.8 \frac{\mathrm{mg}}{\mathrm{ml}}\right)-(0.05 \mathrm{ml})\left(1.8 \frac{\mathrm{mg}}{\mathrm{ml}}\right)-(4.95 \mathrm{ml})\left(0.34 \frac{\mathrm{mg}}{\mathrm{ml}}\right) \\
& =7.2 \mathrm{mg}
\end{aligned}
$$

The amount of penicillin $\mathrm{G}$ in the water pool, $P_{w p}$;

$$
\begin{aligned}
P_{w p} & =[P]_{a q . i} V_{w t}=\left(1.8 \frac{\mathrm{mg}}{\mathrm{ml}}\right)(0.05 \mathrm{ml}) \\
& =0.09 \mathrm{mg}
\end{aligned}
$$

The final total mass of penicillin $\mathrm{G}$ in the reverse micelle organic phase, $[P]_{r m+o f f}$;

$$
\begin{aligned}
P_{m+o . f} & =P_{s . f}+P_{w p}=7.2 \mathrm{mg}+0.09 \mathrm{mg} \\
& =7.29 \mathrm{mg}
\end{aligned}
$$

The final concentration of penicillin $\mathrm{G}$ in the reverse micelles, $[P]_{r m+o f}$;

$$
\begin{aligned}
{[P]_{r+o . f} } & =\frac{P_{r m+o . f}}{V_{r m+o . f}}=\frac{7.29 \mathrm{mg}}{5.05 \mathrm{ml}} \\
& =\underline{1.44 \mathrm{mg} / \mathrm{ml}}
\end{aligned}
$$


Appendix 2

Experimental Results for Forward Extraction 


\section{2-1 Partition coefficient of forward extraction}

a) $[S]=88 \mathrm{~g} / \mathrm{l}, \mathrm{pH}=7.6$

Parameters:

Density of water, $\rho_{w}=1.0 \mathrm{~g} / \mathrm{ml}$

Volume of titration for blank sample, $V_{\text {titrantblank }}=0.6 \mathrm{ml}$

Molarity of acid, $M_{\text {acid }}=0.05 \mathrm{~mol} / \mathrm{l}$

Final volume of water in the aqueous phase, $V_{a q . f}=4.95 \mathrm{ml}$

Initial volume of water in the aqueous phase, $V_{a q . i}=5.0 \mathrm{ml}$

Final volume of water in the reverse micelles, $V_{r m+o . f}=V_{w . t}=0.05 \mathrm{ml}$

\begin{tabular}{|c|c|c|c|c|c|c|}
\hline $\begin{array}{c}{[P]_{\text {aq. } i}} \\
(\mathrm{mg} / \mathrm{ml})\end{array}$ & $\begin{array}{c}P_{\text {aq.i }} \\
(\mathrm{mg})\end{array}$ & $\begin{array}{c}V_{\text {titant }^{*}} \\
(\mathrm{ml})\end{array}$ & $\% N$ & $\begin{array}{c}P_{\text {aq.j. }} \\
(\mathrm{mg})\end{array}$ & $\begin{array}{c}{[P]_{\text {aq. }}} \\
(\mathrm{mg} / \mathrm{ml})\end{array}$ & $\begin{array}{c}{[P]_{r m+o . f}} \\
(\mathrm{mg} / \mathrm{ml})\end{array}$ \\
\hline 0 & 0.0 & $0.6(0.00)$ & 0.000 & 0.00 & 0.00 & 0.00 \\
1.8 & 8.9 & $3.0(0.08)$ & 0.034 & 1.68 & 0.33 & 1.44 \\
3.6 & 17.8 & $5.4(0.08)$ & 0.067 & 3.36 & 0.67 & 2.89 \\
7.1 & 35.6 & $9.9(0.16)$ & 0.131 & 6.51 & 1.31 & 5.73 \\
10.7 & 53.5 & $14.8(0.24)$ & 0.201 & 9.94 & 2.00 & 8.62 \\
14.3 & 71.3 & $19.5(0.24)$ & 0.267 & 13.23 & 2.67 & 11.53 \\
17.8 & 89.1 & $24.0(0.33)$ & 0.331 & 16.39 & 3.31 & 14.37 \\
21.4 & 106.9 & $27.9(0.41)$ & 0.386 & 19.12 & 3.86 & 17.40 \\
24.9 & 124.7 & $32.5(0.41)$ & 0.451 & 22.34 & 4.51 & 20.22 \\
28.5 & 142.7 & $37.0(0.49)$ & 0.515 & 25.49 & 5.15 & 23.16 \\
32.1 & 160.4 & $41.9(0.49)$ & 0.584 & 28.93 & 5.84 & 26.05 \\
35.6 & 178.2 & $46.5(0.49)$ & 0.649 & 32.15 & 6.49 & 28.88 \\
\hline
\end{tabular}

* Standard deviation in ( ), at least 3 repeat tests. 
b) $[S]=267 \mathrm{~g} / \mathrm{l}, \mathrm{pH}=7.6$

\section{Parameters:}

Density of water, $\rho_{w}=1.0 \mathrm{~g} / \mathrm{ml}$

Volume of titration for blank sample, $V_{\text {titrantblank }}=0.6 \mathrm{ml}$

Molarity of acid, $M_{\text {acid }}=0.05 \mathrm{~mol} / \mathrm{l}$

Final volume of water in the aqueous phase, $V_{a q . f}=4.5 \mathrm{ml}$

Initial volume of water in the aqueous phase, $V_{a q . i}=5.0 \mathrm{ml}$

Final volume of water in the reverse micelles, $V_{r m+o . f}=V_{w . t}=0.5 \mathrm{ml}$

\begin{tabular}{|c|c|c|c|c|c|c|}
\hline $\begin{array}{c}{[P]_{\text {aq. } i}} \\
(\mathrm{mg} / \mathrm{ml})\end{array}$ & $\begin{array}{c}P_{\text {aq. } i} \\
(\mathrm{mg})\end{array}$ & $\begin{array}{c}V_{\text {titrant }}{ }^{*} \\
(\mathrm{ml})\end{array}$ & $\% N$ & $\begin{array}{c}P_{\text {aq. } f} \\
(\mathrm{mg})\end{array}$ & $\begin{array}{c}{[P]_{\text {aq. } f}} \\
(\mathrm{mg} / \mathrm{ml})\end{array}$ & $\begin{array}{c}{[P]_{m++o . f}} \\
(\mathrm{mg} / \mathrm{ml})\end{array}$ \\
\hline 0 & 0.0 & $0.6(0.00)$ & 0.000 & 0.00 & 0.00 & 0.00 \\
1.8 & 8.9 & $2.1(0.08)$ & 0.024 & 1.08 & 0.24 & 1.43 \\
3.6 & 17.8 & $3.8(0.16)$ & 0.049 & 2.24 & 0.49 & 2.86 \\
7.1 & 35.6 & $6.8(0.16)$ & 0.096 & 4.34 & 0.96 & 5.66 \\
10.7 & 53.5 & $9.6(0.24)$ & 0.140 & 6.30 & 1.40 & 8.58 \\
14.3 & 71.3 & $13.0(0.33)$ & 0.193 & 8.68 & 1.93 & 11.42 \\
17.8 & 89.1 & $16.0(0.41)$ & 0.239 & 10.78 & 2.39 & 14.22 \\
21.4 & 106.9 & $18.9(0.41)$ & 0.284 & 12.81 & 2.84 & 17.12 \\
24.9 & 124.7 & $21.4(0.49)$ & 0.323 & 14.57 & 3.23 & 19.98 \\
28.5 & 142.7 & $24.6(0.49)$ & 0.373 & 16.81 & 3.73 & 22.85 \\
32.1 & 160.4 & $27.5(0.57)$ & 0.418 & 18.84 & 4.18 & 25.75 \\
35.6 & 178.2 & $30.4(0.57)$ & 0.463 & 20.87 & 4.63 & 28.56 \\
\hline
\end{tabular}

* Standard deviation in ( ), at least 3 repeat tests. 
c) $[S]=445 \mathrm{~g} / \mathrm{l}, \mathrm{pH}=7.6$

Parameters:

Density of water, $\rho_{w}=1.0 \mathrm{~g} / \mathrm{ml}$

Volume of titration for blank sample, $V_{\text {titrantblank }}=0.6 \mathrm{ml}$

Molarity of acid, $M_{\text {acid }}=0.05 \mathrm{~mol} / \mathrm{l}$

Final volume of water in the aqueous phase, $V_{a q . f}=3.0 \mathrm{ml}$

Initial volume of water in the aqueous phase, $V_{\text {aq. } i}=5.0 \mathrm{ml}$

Final volume of water in the reverse micelles, $V_{r m+o f}=V_{w . t}=2.0 \mathrm{ml}$

\begin{tabular}{|c|c|c|c|c|c|c|}
\hline $\begin{array}{c}{[P]_{\text {aq.i }}} \\
(\mathrm{mg} / \mathrm{ml})\end{array}$ & $\begin{array}{c}P_{\text {aq. } i} \\
(\mathrm{mg})\end{array}$ & $\begin{array}{c}V_{\text {titrant }}{ }^{*} \\
(\mathrm{ml})\end{array}$ & $\% N$ & $\begin{array}{c}P_{a q . f} \\
(\mathrm{mg})\end{array}$ & $\begin{array}{c}{[P]_{\text {aq. }}} \\
(\mathrm{mg} / \mathrm{ml})\end{array}$ & $\begin{array}{c}{[P]_{m+o . f}} \\
(\mathrm{mg} / \mathrm{ml})\end{array}$ \\
\hline 0 & 0.0 & $0.6(0.00)$ & 0.000 & 0.00 & 0.00 & 0 \\
1.8 & 8.9 & $1.4(0.08)$ & 0.018 & 0.56 & 0.18 & 1.20 \\
3.6 & 17.8 & $2.3(0.16)$ & 0.039 & 1.19 & 0.39 & 2.40 \\
7.1 & 35.6 & $3.9(0.24)$ & 0.077 & 2.31 & 0.77 & 4.74 \\
10.7 & 53.5 & $5.5(0.33)$ & 0.114 & 3.43 & 1.14 & 7.15 \\
14.3 & 71.3 & $7.0(0.41)$ & 0.149 & 4.48 & 1.49 & 9.57 \\
17.8 & 89.1 & $8.9(0.49)$ & 0.193 & 5.81 & 1.93 & 11.88 \\
21.4 & 106.9 & $10.0(0.49)$ & 0.219 & 6.58 & 2.19 & 14.34 \\
24.9 & 124.7 & $12.0(0.57)$ & 0.266 & 7.98 & 2.66 & 16.64 \\
28.5 & 142.7 & $13.8(0.57)$ & 0.308 & 9.24 & 3.08 & 19.03 \\
32.1 & 160.4 & $15.4(0.65)$ & 0.345 & 10.36 & 3.45 & 21.44 \\
35.6 & 178.2 & $16.5(0.65)$ & 0.371 & 11.13 & 3.71 & 23.83 \\
\hline
\end{tabular}

* Standard deviation in ( ), at least 3 repeat tests. 
d) $[S]=88 \mathrm{~g} / \mathrm{l}, \mathrm{pH}=1.65$

Parameters:

Density of water, $\rho_{w}=1.0 \mathrm{~g} / \mathrm{ml}$

Volume of titration for blank sample, $V_{\text {titrantblank }}=0.6 \mathrm{ml}$

Molarity of acid, $M_{\text {acid }}=0.05 \mathrm{~mol} / \mathrm{l}$

Final volume of water in the aqueous phase, $V_{a q . f}=4.95 \mathrm{ml}$

Initial volume of water in the aqueous phase, $V_{a q . i}=5.0 \mathrm{ml}$

Final volume of water in the reverse micelles, $V_{r m+o . f}=V_{w . t}=0.05 \mathrm{ml}$

\begin{tabular}{|c|c|c|c|c|c|c|}
\hline $\begin{array}{c}{[P]_{\text {aq.i }}} \\
(\mathrm{mg} / \mathrm{ml})\end{array}$ & $\begin{array}{c}P_{\text {aq.i }} \\
(\mathrm{mg})\end{array}$ & $\begin{array}{c}V_{\text {titrant }}{ }^{*} \\
(\mathrm{ml})\end{array}$ & $\% N$ & $\begin{array}{c}P_{\text {aq.f }} \\
(\mathrm{mg})\end{array}$ & $\begin{array}{c}{[P]_{\text {aq. } f}} \\
(\mathrm{mg} / \mathrm{ml})\end{array}$ & $\begin{array}{c}{[P]_{m+o . f}} \\
(\mathrm{mg} / \mathrm{ml})\end{array}$ \\
\hline 0 & 0.0 & $0.6(0.00)$ & 0.000 & 0.00 & 0.00 & 0.00 \\
1.8 & 8.9 & $2.4(0.16)$ & 0.025 & 1.26 & 0.25 & 1.53 \\
3.6 & 17.8 & $4.1(0.24)$ & 0.050 & 2.45 & 0.50 & 3.08 \\
7.1 & 35.6 & $7.4(0.33)$ & 0.096 & 4.76 & 0.96 & 6.09 \\
10.7 & 53.5 & $11.6(0.33)$ & 0.156 & 7.71 & 1.56 & 9.07 \\
\hline
\end{tabular}

* Standard deviation in ( ), at least 3 repeat tests.

e) $[S]=267 \mathrm{~g} / \mathrm{l}, \mathrm{pH}=1.65$

Parameters:

Density of water, $\rho_{w}=1.0 \mathrm{~g} / \mathrm{ml}$

Volume of titration for blank sample, $V_{\text {titrantblank }}=0.6 \mathrm{ml}$

Molarity of acid, $M_{\text {acid }}=0.5 \mathrm{~mol} / \mathrm{l}$

Final volume of water in the aqueous phase, $V_{a q . f}=4.5 \mathrm{ml}$

Initial volume of water in the aqueous phase, $V_{a q . i}=5.0 \mathrm{ml}$

Final volume of water in the reverse micelles, $V_{r m+o . f}=V_{w . t}=0.5 \mathrm{ml}$ 


\begin{tabular}{|c|c|c|c|c|c|c|}
\hline $\begin{array}{c}{[P]_{a q . i}} \\
(\mathrm{mg} / \mathrm{ml})\end{array}$ & $\begin{array}{c}P_{\text {aq.i }} \\
(\mathrm{mg})\end{array}$ & $\begin{array}{c}V_{\text {tirant }}{ }^{*} \\
(\mathrm{ml})\end{array}$ & $\% N$ & $\begin{array}{c}P_{a q . f} \\
(\mathrm{mg})\end{array}$ & $\begin{array}{c}{[P]_{\text {aq.f }}} \\
(\mathrm{mg} / \mathrm{ml})\end{array}$ & $\begin{array}{c}{[P]_{m+o . f}} \\
(\mathrm{mg} / \mathrm{ml})\end{array}$ \\
\hline 0 & 0.0 & $0.6(0.16)$ & 0.000 & 0.00 & 0.00 & 0.00 \\
1.8 & 8.9 & $1.9(0.24)$ & 0.020 & 0.91 & 0.20 & 1.47 \\
3.6 & 17.8 & $3.3(0.33)$ & 0.042 & 1.89 & 0.42 & 2.93 \\
7.1 & 35.6 & $5.9(0.41)$ & 0.083 & 3.71 & 0.83 & 5.78 \\
10.7 & 53.5 & $8.8(0.49)$ & 0.128 & 5.74 & 1.28 & 8.68 \\
\hline
\end{tabular}

* Standard deviation in ( ), at least 3 repeat tests.

\section{f) $[S]=445 \mathrm{~g} / \mathrm{l}, \mathrm{pH}=1.65$}

Parameters:

Density of water, $\rho_{w}=1.0 \mathrm{~g} / \mathrm{ml}$

Volume of titration for blank sample, $V_{\text {titrantblank }}=0.6 \mathrm{ml}$

Molarity of acid, $M_{\text {acid }}=0.5 \mathrm{~mol} / \mathrm{l}$

Final volume of water in the aqueous phase, $V_{a q . f}=3.0 \mathrm{ml}$

Initial volume of water in the aqueous phase, $V_{a q . i}=5.0 \mathrm{ml}$

Final volume of water in the reverse micelles, $V_{r m+o . f}=V_{w . t}=2.0 \mathrm{ml}$

\begin{tabular}{|c|c|c|c|c|c|c|}
\hline $\begin{array}{c}{[P]_{\text {aq. } i}} \\
(\mathrm{mg} / \mathrm{ml})\end{array}$ & $\begin{array}{c}P_{\text {aq.i }} \\
(\mathrm{mg})\end{array}$ & $\begin{array}{c}V_{\text {titrant }}{ }^{*} \\
(\mathrm{ml})\end{array}$ & $\% N$ & $\begin{array}{c}P_{\text {aq. }} \\
(\mathrm{mg})\end{array}$ & $\begin{array}{c}{[P]_{\text {aq. }}} \\
(\mathrm{mg} / \mathrm{ml})\end{array}$ & $\begin{array}{c}{[P]_{r m+o . f}} \\
(\mathrm{mg} / \mathrm{ml})\end{array}$ \\
\hline 0 & 0.0 & $0.6(0.24)$ & 0.000 & 0.00 & 0.00 & 0.00 \\
1.8 & 8.9 & $1.3(0.24)$ & 0.016 & 0.49 & 0.16 & 1.22 \\
3.6 & 17.8 & $2.0(0.33)$ & 0.033 & 0.98 & 0.33 & 2.43 \\
7.1 & 35.6 & $3.4(0.49)$ & 0.065 & 1.96 & 0.65 & 4.79 \\
10.7 & 53.5 & $4.9(0.49)$ & 0.100 & 3.01 & 1.00 & 7.21 \\
\hline
\end{tabular}

* Standard deviation in ( ), at least 3 repeat tests. 


\section{2-2 Additional volume of organic phase, $V_{w . t}$}

Parameters:

Initial volume of water in the aqueous phase, $V_{\text {aq. } i}=5.0 \mathrm{ml}$

$\mathrm{pH}=7.6$

$[\mathrm{KCl}]=10 \mathrm{~g} / 1$

\begin{tabular}{|c|c|c|c|}
\hline$[S](\mathrm{g} / \mathrm{l})$ & $V_{w, t}{ }^{*}(\mathrm{ml})$ & $V_{\text {aq, }}(\mathrm{ml})$ & $V_{\text {rm+o.f }}(\mathrm{ml})$ \\
\hline 44 & $0.00(0.004)$ & 5.00 & 5.00 \\
89 & $0.05(0.008)$ & 4.95 & 5.09 \\
222 & $0.25(0.012)$ & 4.75 & 5.25 \\
267 & $0.50(0.024)$ & 4.50 & 4.50 \\
333 & $1.15(0.041)$ & 3.85 & 6.15 \\
445 & $2.00(0.061)$ & 3.00 & 7.00 \\
\hline
\end{tabular}

* Standard deviation in ( ), at least 3, repeat tests.

\section{2-3 Critical micelle concentration (CMC)}

Parameters:

Initial volume of water in the aqueous phase, $V_{a q . i}=5.0 \mathrm{ml}$

$\mathrm{pH}=7.6$

$[\mathrm{KCl}]=10 \mathrm{~g} / \mathrm{l}$

a) Without penicillin G

\begin{tabular}{|c|c|}
\hline$[S](\mathrm{g} / \mathrm{l})$ & Surface tension* $(\mathrm{mN} / \mathrm{m})$ \\
\hline 0 & $21.50(0.61)$ \\
4 & $15.50(0.61)$ \\
22 & $10.50(0.41)$ \\
44 & $10.00(0.16)$ \\
89 & $10.00(0.16)$ \\
133 & $10.00(0.16)$ \\
222 & $10.00(0.16)$ \\
\hline
\end{tabular}

* Standard deviation in ( ), at least 3 repeat tests. 
a) With penicillin G

\begin{tabular}{|c|c|}
\hline$[S](\mathrm{g} / \mathrm{l})$ & Surface tension* $(\mathrm{mN} / \mathrm{m})$ \\
\hline 0 & $21.50(0.61)$ \\
4 & $21.00(0.61)$ \\
22 & $20.80(0.61)$ \\
44 & $15.50(0.41)$ \\
89 & $10.50(0.41)$ \\
133 & $10.00(0.16)$ \\
222 & $10.00(0.16)$ \\
\hline
\end{tabular}

* Standard deviation in ( ), at least 3 repeat tests.

\section{2-4 Fractional transfer of penicillin and water, $t_{a}$ and $t_{w}$}

$t_{a}$ and $t_{w}$ values are calculated using equations (4.1) and (4.2). $[P]_{a q . f}$ is taken from results of the partition coefficient (Appendix A2-1).

a) $\underline{\mathrm{pH}}=7.6,[S]=88 \mathrm{~g} / 1$

Parameters:

Initial volume of water in the aqueous phase, $V_{a q . i}=5.0 \mathrm{ml}$

Final volume of water in the aqueous phase, $V_{a q f}=4.95 \mathrm{ml}$

\begin{tabular}{|c|c|c|c|}
\hline$[P]_{a q . i}(\mathrm{mg} / \mathrm{ml})$ & {$[P]_{a q . j}(\mathrm{mg} / \mathrm{ml})$} & $t_{a}$ & $t_{w}$ \\
\hline 3.6 & 0.6793 & 0.010 & 0.813 \\
7.1 & 1.3161 & 0.010 & 0.816 \\
21.40 & 3.8634 & 0.010 & 0.821 \\
32.10 & 5.8446 & 0.010 & 0.820 \\
\hline
\end{tabular}


b) $\underline{\mathrm{pH}}=7.6,[S]=267 \mathrm{~g} / 1$

Parameters:

Initial volume of water in theaqueous phase, $V_{a q . i}=5.0 \mathrm{ml}$

Final volume of water in the aqueous phase, $V_{a q . f}=4.5 \mathrm{ml}$

\begin{tabular}{|c|c|c|c|}
\hline$[P]_{\text {aq. } .}(\mathrm{mg} / \mathrm{ml})$ & {$[P]_{\text {aqf. }}(\mathrm{mg} / \mathrm{ml})$} & $t_{a}$ & $t_{w}$ \\
\hline 3.6 & 0.4981 & 0.100 & 0.875 \\
7.1 & 0.9651 & 0.100 & 0.878 \\
21.40 & 2.8487 & 0.100 & 0.880 \\
32.10 & 4.1874 & 0.100 & 0.883 \\
\hline
\end{tabular}

c) $\mathrm{pH}=7.6,[S]=333 \mathrm{~g} / \mathrm{l}$

Parameters:

Initial volume of water in aqueous phase, $V_{a q . i}=5.0 \mathrm{ml}$

Final volume of water in the aqueous phase, $V_{a q . f}=3.85 \mathrm{ml}$

\begin{tabular}{|c|c|c|c|}
\hline$[P]_{\text {aq. }}(\mathrm{mg} / \mathrm{ml})$ & {$[P]_{\text {aq. }}(\mathrm{mg} / \mathrm{ml})$} & $t_{a}$ & $t_{w}$ \\
\hline 3.6 & 0.4670 & 0.250 & 0.903 \\
7.1 & 0.8406 & 0.250 & 0.911 \\
21.40 & 2.2790 & 0.250 & 0.920 \\
32.10 & 3.3437 & 0.250 & 0.922 \\
\hline
\end{tabular}

d) $\underline{\mathrm{H}}=7.6,[S]=445 \mathrm{~g} / 1$

Parameters:

Initial volume of water in the aqueous phase, $V_{a q . i}=5.0 \mathrm{ml}$

Final volume of water in the aqueous phase, $V_{a q . f}=3.0 \mathrm{ml}$ 


\begin{tabular}{|c|c|c|c|}
\hline$[P]_{a p . i}(\mathrm{mg} / \mathrm{ml})$ & {$[P]_{a q, f}(\mathrm{mg} / \mathrm{ml})$} & $t_{a}$ & $t_{w}$ \\
\hline 3.6 & 0.3970 & 0.400 & 0.934 \\
7.1 & 0.7706 & 0.400 & 0.935 \\
21.40 & 2.1949 & 0.400 & 0.938 \\
32.10 & 3.4558 & 0.400 & 0.935 \\
\hline
\end{tabular}

e) $\underline{\mathrm{pH}}=1.65,[S]=88 \mathrm{~g} / 1$

Parameters:

Initial volume of water in the aqueous phase, $V_{a q . i}=5.0 \mathrm{ml}$

Final volume of water in the aqueous phase, $V_{a q . f}=4.95 \mathrm{ml}$

\begin{tabular}{|c|c|c|c|}
\hline$[P]_{\text {aq. } .}(\mathrm{mg} / \mathrm{ml})$ & {$[P]_{\text {aq. }}(\mathrm{mg} / \mathrm{ml})$} & $t_{a}$ & $t_{w}$ \\
\hline 3.6 & 0.4953 & 0.010 & 0.864 \\
7.1 & 0.9623 & 0.010 & 0.866 \\
\hline
\end{tabular}

\section{f) $\mathrm{pH}=1.65,[S]=267 \mathrm{~g} / \mathrm{l}$}

Parameters:

Initial volume of water in the aqueous phase, $V_{a q . i}=5.0 \mathrm{ml}$

Final volume of water in the aqueous phase, $V_{a q f}=4.5 \mathrm{ml}$

\begin{tabular}{|c|c|c|c|}
\hline$[P]_{a q, i}(\mathrm{mg} / \mathrm{ml})$ & {$[P]_{\text {aq. }}(\mathrm{mg} / \mathrm{ml})$} & $t_{a}$ & $t_{w}$ \\
\hline 3.6 & 0.4203 & 0.100 & 0.895 \\
7.1 & 0.8250 & 0.100 & 0.895 \\
\hline
\end{tabular}




\section{g) $\mathrm{pH}=1.65,[S]=333 \mathrm{~g} / 1$}

\section{Parameters:}

Initial volume of water in the aqueous phase, $V_{a q . i}=5.0 \mathrm{ml}$

Final volume of water in the aqueous phase, $V_{a q . f}=3.85 \mathrm{ml}$

\begin{tabular}{|c|c|r|r|}
\hline $\begin{array}{c}{[P]_{\text {aq. }}} \\
(\mathrm{mg} / \mathrm{ml})\end{array}$ & $\begin{array}{c}{[P]_{\text {aq. }}} \\
(\mathrm{mg} / \mathrm{ml})\end{array}$ & $t_{a}$ & \multicolumn{2}{|c|}{$t_{w}$} \\
\hline 3.6 & 0.3736 & 0.250 & 0.922 \\
7.1 & 0.6351 & 0.250 & 0.933 \\
\hline
\end{tabular}

h) $\mathrm{pH}=1.65,[S]=445 \mathrm{~g} / 1$

Parameters:

Initial volume of water in the aqueous phase, $V_{a q . i}=5.0 \mathrm{ml}$

Final volume of water in the aqueous phase, $V_{a q . f}=3.0 \mathrm{ml}$

\begin{tabular}{|c|c|c|c|}
\hline$[P]_{\text {aq. } i}(\mathrm{mg} / \mathrm{ml})$ & {$[P]_{\text {aq. }}(\mathrm{mg} / \mathrm{ml})$} & $t_{a}$ & $t_{w}$ \\
\hline 3.6 & 0.3269 & 0.400 & 0.946 \\
7.1 & 0.6538 & 0.400 & 0.945 \\
\hline
\end{tabular}




\section{2-5 Effect of surfactant concentration}

Parameters:

Density of water, $\rho_{w}=1.0 \mathrm{~g} / \mathrm{ml}$

Volume of titration for blank sample, $V_{\text {titrantblank }}=0.6 \mathrm{ml}$

Molarity of acid, $M_{\text {acid }}=0.5 \mathrm{~mol} / 1$

Initial volume of water in the aqueous phase, $V_{a q . i}=5.0 \mathrm{ml}$

Volume of water transfer into the reverse micelle phase, $V_{w . t}=2.0 \mathrm{ml}$

Initial concentration of penicillin $\mathrm{G}$ in the aqueous phase, $[P]_{a q . f}=3.6 \mathrm{~g} / 1$

\begin{tabular}{|c|c|c|c|c|c|c|}
\hline $\begin{array}{c}{[S]} \\
(\mathrm{g} / \mathrm{l})\end{array}$ & $\begin{array}{c}V_{\text {titrant }}{ }^{*} \\
(\mathrm{ml})\end{array}$ & $\% N$ & $\begin{array}{c}V_{\text {w.t }} \\
(\mathrm{ml})\end{array}$ & $\begin{array}{c}P_{\text {aq.f }} \\
(\mathrm{mg})\end{array}$ & $\begin{array}{c}{[P]_{\text {aqf. }}} \\
(\mathrm{mg} / \mathrm{ml})\end{array}$ & $\begin{array}{c}{[P]_{m+o . f}} \\
(\mathrm{mg} / \mathrm{ml})\end{array}$ \\
\hline 0 & $0.6(0.00)$ & 0.000 & 0.0 & 0.00 & 0.00 & 0.00 \\
88 & $5.4(0.08)$ & 0.067 & 0.05 & 3.36 & 0.67 & 2.89 \\
222 & $4.4(0.08)$ & 0.056 & 0.25 & 2.66 & 0.56 & 2.92 \\
333 & $3.1(0.16)$ & 0.045 & 1.15 & 1.75 & 0.45 & 2.64 \\
445 & $2.2(0.16)$ & 0.037 & 2.00 & 1.12 & 0.37 & 2.41 \\
\hline
\end{tabular}

* Standard deviation in ( ), at least 3 repeat tests.

\section{2-6 Effect of $\mathrm{pH}$}

a) $[S]=88 \mathrm{~g} / \mathrm{l}$

Parameters:

Density of water, $\rho_{w}=1.0 \mathrm{~g} / \mathrm{ml}$

Volume of titration for blank sample, $V_{\text {titrantblank }}=0.6 \mathrm{ml}$

Molarity of acid, $M_{\text {acid }}=0.05 \mathrm{~mol} / \mathrm{l}$

Final volume of water in the aqueous phase, $V_{a q f}=4.95 \mathrm{ml}$

Initial volume of water in the aqueous phase, $V_{a q . i}=5.0 \mathrm{ml}$

Final volume of water in the reverse micelles, $V_{r m+o . f}=V_{w . t}=0.05 \mathrm{mI}$

Initial concentration of penicillin $\mathrm{G}$ the in aqueous phase, $[P]_{\text {aq. }}=3.6 \mathrm{~g} / \mathrm{l}$ 


\begin{tabular}{|c|c|c|c|c|c|}
\hline $\mathrm{pH}$ & $\begin{array}{c}V_{\text {titrant }}{ }^{*} \\
(\mathrm{ml})\end{array}$ & $\% N$ & $\begin{array}{c}P_{\text {aq. }} \\
(\mathrm{mg})\end{array}$ & $\begin{array}{c}{[P]_{\text {aqf }}} \\
(\mathrm{mg} / \mathrm{ml})\end{array}$ & $\begin{array}{c}{[P]_{r m+o . f}} \\
(\mathrm{mg} / \mathrm{ml})\end{array}$ \\
\hline 0.85 & $5.35(0.016)$ & 0.090 & 4.48 & 0.90 & 2.90 \\
1.65 & $4.1(0.08)$ & 0.063 & 3.15 & 0.636 & 2.94 \\
2.4 & $5.4(0.08)$ & 0.067 & 3.36 & 0.67 & 2.89 \\
3.6 & $6.0(0.16)$ & 0.076 & 3.78 & 0.76 & 2.81 \\
4.5 & $6.2(0.16)$ & 0.079 & 3.92 & 0.79 & 2.78 \\
5.5 & $6.3(0.08)$ & 0.080 & 3.99 & 0.80 & 2.77 \\
6.4 & $5.8(0.16)$ & 0.073 & 3.64 & 0.73 & 2.84 \\
7.6 & $5.4(0.16)$ & 0.067 & 3.36 & 0.67 & 2.89 \\
8.5 & $5.6(0.16)$ & 0.070 & 3.50 & 0.70 & 2.87 \\
9.8 & $5.8(0.16)$ & 0.073 & 3.64 & 0.73 & 2.84 \\
\hline
\end{tabular}

* Standard deviation in ( ), at least 3 repeat tests.

b) $[S]=222 \mathrm{~g} / \mathrm{l}$

Parameters:

Density of water, $\rho_{w}=1.0 \mathrm{~g} / \mathrm{ml}$

Volume of titration for blank sample, $V_{\text {titrantblank }}=0.6 \mathrm{ml}$

Molarity of acid, $M_{a c i d}=0.05 \mathrm{~mole} / \mathrm{l}$

Final volume of water in the aqueous phase, $V_{a q . f}=4.75 \mathrm{ml}$

Initial volume of water in the aqueous phase, $V_{a q . i}=5.0 \mathrm{ml}$

Final volume of water in the reverse micelles, $V_{r m+o . f}=V_{w . t}=0.25 \mathrm{ml}$

Initial concentration of penicillin $\mathrm{G}$ in the aqueous phase, $[P]_{\text {aq. }}=3.6 \mathrm{~g} / 1$

\begin{tabular}{|c|c|c|c|c|c|}
\hline $\mathrm{pH}$ & $\begin{array}{c}V_{\text {titrant }} * \\
(\mathrm{ml})\end{array}$ & $\% N$ & $\begin{array}{c}P_{\text {aq.f }} \\
(\mathrm{mg})\end{array}$ & $\begin{array}{c}{[P]_{\text {aq.f }}} \\
(\mathrm{mg} / \mathrm{ml})\end{array}$ & $\begin{array}{c}{[P]_{\text {rnto.f }}} \\
(\mathrm{mg} / \mathrm{ml})\end{array}$ \\
\hline 0.85 & $4.4(0.08)$ & 0.056 & 2.66 & 0.56 & 2.92 \\
1.6 & $4.2(0.08)$ & 0.053 & 2.52 & 0.53 & 2.95 \\
2.4 & $4.5(0.08)$ & 0.058 & 2.73 & 0.58 & 2.91 \\
3.6 & $4.5(0.08)$ & 0.058 & 2.73 & 0.58 & 2.91 \\
4.5 & $4.7(0.16)$ & 0.060 & 2.87 & 0.60 & 2.88 \\
5.5 & $4.4(0.08)$ & 0.056 & 2.66 & 0.56 & 2.92 \\
6.4 & $4.3(0.16)$ & 0.055 & 2.59 & 0.55 & 2.93 \\
7.6 & $4.4(0.16)$ & 0.056 & 2.66 & 0.56 & 2.92 \\
8.5 & $4.7(0.16)$ & 0.060 & 2.87 & 0.60 & 2.88 \\
9.8 & $4.8(0.16)$ & 0.062 & 2.94 & 0.62 & 2.87 \\
\hline
\end{tabular}

* Standard deviation in ( ), at least 3 repeat tests. 
c) $[S]=445 \mathrm{~g} / 1$

Parameters:

Density of water, $\rho_{w}=1.0 \mathrm{~g} / \mathrm{ml}$

Volume of titration for blank sample, $V_{\text {titrantblank }}=0.6 \mathrm{ml}$

Molarity of acid, $M_{\text {acid }}=0.05 \mathrm{~mol} / \mathrm{l}$

Final volume of water in the aqueous phase, $V_{a q . f}=3.0 \mathrm{ml}$

Initial volume of water in the aqueous phase, $V_{a q . i}=5.0 \mathrm{ml}$

Final volume of water in the reverse micelles, $V_{r m+o f}=V_{w . t}=2.0 \mathrm{ml}$

Initial concentration of penicillin $G$ in the aqueous phase, $[P]_{a q . f}=3.6 \mathrm{~g} / 1$

\begin{tabular}{|c|c|c|c|c|c|}
\hline $\mathrm{pH}$ & $\begin{array}{c}V_{\text {titrant }}{ }^{*} \\
(\mathrm{ml})\end{array}$ & $\% N$ & $\begin{array}{c}P_{\text {aq. }} \\
(\mathrm{mg})\end{array}$ & $\begin{array}{c}{[P]_{a q . f}} \\
(\mathrm{mg} / \mathrm{ml})\end{array}$ & $\begin{array}{c}{[P]_{m+o . f}} \\
(\mathrm{mg} / \mathrm{ml})\end{array}$ \\
\hline 0.85 & $2.2(0.08)$ & 0.037 & 1.12 & 0.37 & 2.41 \\
1.6 & $2.0(0.08)$ & 0.033 & 0.98 & 0.33 & 2.43 \\
2.4 & $2.2(0.08)$ & 0.037 & 1.12 & 0.37 & 2.41 \\
3.6 & $2.5(0.16)$ & 0.044 & 1.33 & 0.44 & 2.38 \\
4.5 & $2.5(0.16)$ & 0.044 & 1.33 & 0.44 & 2.38 \\
5.5 & $2.4(0.16)$ & 0.042 & 1.26 & 0.42 & 2.39 \\
6.4 & $2.3(0.16)$ & 0.040 & 1.19 & 0.40 & 2.40 \\
7.6 & $2.2(0.16)$ & 0.037 & 1.12 & 0.37 & 2.41 \\
8.5 & $2.3(0.16)$ & 0.040 & 1.19 & 0.40 & 2.40 \\
9.8 & $2.3(0.16)$ & 0.040 & 1.19 & 0.40 & 2.40 \\
\hline
\end{tabular}

* Standard deviation in (), at least 3 repeat tests. 


\section{2-7 Effect of salt concentration, $[\mathrm{KCl}]$}

\section{a) $[S]=222 \mathrm{~g} / \mathrm{l}$}

Parameters:

Density of water, $\rho_{w}=1.0 \mathrm{~g} / \mathrm{ml}$

Volume of titration for blank sample, $V_{\text {titrantblank }}=0.6 \mathrm{ml}$

Molarity of acid, $M_{\text {acid }}=0.05 \mathrm{~mol} / \mathrm{l}$

Final volume of water in the aqueous phase, $V_{a q . f}=4.75 \mathrm{ml}$

Initial volume of water in the aqueous phase, $V_{a q . i}=5.0 \mathrm{ml}$

Final volume of water in the reverse micelles, $V_{r m+o . f}=V_{w . t}=0.25 \mathrm{ml}$

Initial concentration of penicillin $\mathrm{G}$ in the aqueous phase, $[P]_{a q . f}=3.6 \mathrm{~g} / \mathrm{l}$

\begin{tabular}{|c|c|c|c|c|c|}
\hline $\begin{array}{c}{[\mathrm{KCl}]} \\
(\mathrm{g} / \mathrm{l})\end{array}$ & $\begin{array}{c}V_{\text {titrant }} * \\
(\mathrm{ml})\end{array}$ & $\% N$ & $\begin{array}{c}P_{\text {aq. }} \\
(\mathrm{mg})\end{array}$ & $\begin{array}{c}{[P]_{a g . f}} \\
(\mathrm{mg} / \mathrm{ml})\end{array}$ & $\begin{array}{c}{[P]_{m+o . f}} \\
(\mathrm{mg} / \mathrm{ml})\end{array}$ \\
\hline 1 & $25.0(1.63)$ & 0.360 & 17.09 & 3.60 & 0.17 \\
5 & $4.8(0.16)$ & 0.062 & 2.94 & 0.62 & 2.87 \\
10 & $4.0(0.08)$ & 0.050 & 2.38 & 0.50 & 2.97 \\
20 & $9.0(0.40)$ & 0.124 & 5.88 & 1.24 & 2.31 \\
30 & $17.0(0.82)$ & 0.242 & 11.49 & 2.42 & 1.24 \\
50 & $19.4(0.82)$ & 0.277 & 13.17 & 2.77 & 0.92 \\
70 & $21.7(0.82)$ & 0.311 & 14.78 & 3.11 & 0.61 \\
\hline
\end{tabular}

* Standard deviation in ( ), at least 3 repeat tests. 
b) $[S]=267 \mathrm{~g} / \mathrm{l}$

Parameters:

Density of water, $\rho_{w}=1.0 \mathrm{~g} / \mathrm{ml}$

Volume of titration for blank sample, $V_{\text {titrantblank }}=0.6 \mathrm{ml}$

Molarity of acid, $M_{\text {acid }}=0.5 \mathrm{~mole} / 1$

Final volume of water in the aqueous phase, $V_{a q . f}=4.5 \mathrm{ml}$

Initial volume of water in the aqueous phase, $V_{\text {aq. } i}=5.0 \mathrm{ml}$

Final volume of water in the reverse micelles, $V_{r m+o . f}=V_{w . t}=0.5 \mathrm{ml}$

Initial concentration of penicillin $\mathrm{G}$ in the aqueous phase, $[P]_{a q . f}=3.6 \mathrm{~g} / 1$

\begin{tabular}{|c|c|c|c|c|c|}
\hline $\begin{array}{l}{[\mathrm{KCl}]} \\
(\mathrm{g} / \mathrm{l})\end{array}$ & $\begin{array}{c}V_{\text {titrant }} * \\
(\mathrm{ml})\end{array}$ & $\% N$ & $\begin{array}{l}P_{a q f f} \\
(\mathrm{mg})\end{array}$ & $\begin{array}{c}{[P]_{a q . f}} \\
(\mathrm{mg} / \mathrm{ml})\end{array}$ & $\begin{array}{l}{[P]_{m+o f f}} \\
(\mathrm{mg} / \mathrm{ml})\end{array}$ \\
\hline 1 & $23.7(1.63)$ & 0.360 & 16.18 & 3.60 & 0.33 \\
\hline 5 & $6.0(0.08)$ & 0.084 & 3.78 & 0.84 & 2.58 \\
\hline 10 & $3.5(0.16)$ & 0.045 & 2.03 & 0.45 & 2.90 \\
\hline 20 & $7.9(0.24)$ & 0.114 & 5.11 & 1.14 & 2.34 \\
\hline 30 & $16.0(0.40)$ & 0.240 & 10.79 & 2.40 & 1.31 \\
\hline 50 & $18.6(0.82)$ & 0.280 & 12.61 & 2.80 & 0.98 \\
\hline 70 & $20.1(0.82)$ & 0.304 & $\cdot 13.66$ & 3.04 & 0.79 \\
\hline
\end{tabular}

* Standard deviation in ( ), at least 3 repeat tests. 


\section{2-8 Effect of salt type}

a) $\underline{\mathrm{KCl}}$

Parameters:

Density of water, $\rho_{w}=1.0 \mathrm{~g} / \mathrm{ml}$

Volume of titration for blank sample, $V_{\text {titrantblank }}=0.6 \mathrm{ml}$

Molarity of acid, $M_{\text {acid }}=0.05 \mathrm{~mol} / 1$

Final volume of water in the aqueous phase, $V_{a q . f}=4.5 \mathrm{ml}$

Initial volume of water in the aqueous phase, $V_{a q . i}=5.0 \mathrm{ml}$

Final volume of water in the reverse micelles, $V_{r m+o . f}=V_{w . t}=0.5 \mathrm{ml}$

Initial concentration of penicillin $\mathrm{G}$ in the aqueous phase, $[P]_{\text {aq. }}=3.6 \mathrm{~g} / 1$

Surfactant concentration, $[S]=267 \mathrm{~g} / \mathrm{l}$

\begin{tabular}{|c|c|c|c|c|c|}
\hline $\begin{array}{c}{[\mathrm{KCl}]} \\
(\mathrm{g} / \mathrm{l})\end{array}$ & $\begin{array}{c}V_{\text {titramt }}{ }^{*} \\
(\mathrm{ml})\end{array}$ & $\% N$ & $\begin{array}{c}P_{\text {aq. }} \\
(\mathrm{mg})\end{array}$ & $\begin{array}{c}{[P]_{\text {aq. }}} \\
(\mathrm{mg} / \mathrm{ml})\end{array}$ & $\begin{array}{c}{[P]_{m+o . f}} \\
(\mathrm{mg} / \mathrm{ml})\end{array}$ \\
\hline $\mathrm{I}$ & $23.7(1.22)$ & 0.360 & 16.18 & 3.60 & 0.33 \\
5 & $6.0(0.08)$ & 0.084 & 3.78 & 0.84 & 2.58 \\
10 & $3.5(0.16)$ & 0.045 & 2.03 & 0.45 & 2.90 \\
20 & $7.9(0.16)$ & 0.114 & 5.11 & 1.14 & 2.34 \\
30 & $16.0(0.24)$ & 0.240 & 10.79 & 2.40 & 1.31 \\
50 & $18.6(0.40)$ & 0.280 & 12.61 & 2.80 & 0.98 \\
70 & $20.1(0.82)$ & 0.304 & 13.66 & 3.04 & 0.79 \\
\hline
\end{tabular}

* Standard deviation in ( ), at least 3 repeat tests. 
b) $\underline{\mathrm{CaCl}_{2}}$

Parameters:

Density of water, $\rho_{w}=1.0 \mathrm{~g} / \mathrm{ml}$

Volume of titration for blank sample, $V_{\text {titrantblank }}=0.6 \mathrm{ml}$

Molarity of acid, $M_{a c i d}=0.5 \mathrm{~mol} / 1$

Final volume of water in the aqueous phase, $V_{a q . f}=4.5 \mathrm{ml}$

Initial amount of water in the aqueous phase, $V_{a q . i}=5.0 \mathrm{ml}$

Final volume of water in the reverse micelles, $V_{r m+o . f}=V_{w . t}=0.5 \mathrm{ml}$

Initial concentration of penicillin $G$ in the aqueous phase, $[P]_{\text {aq. }}=3.6 \mathrm{~g} / \mathrm{l}$

Surfactant concentration, $[S]=267 \mathrm{~g} / 1$

\begin{tabular}{|c|c|c|c|c|c|}
\hline $\begin{array}{c}{[\mathrm{KCl}]} \\
(\mathrm{g} / \mathrm{l})\end{array}$ & $\begin{array}{c}V_{\text {titrant }} * \\
(\mathrm{ml})\end{array}$ & $\% N$ & $\begin{array}{c}P_{\text {aq. }} \\
(\mathrm{mg})\end{array}$ & $\begin{array}{c}{[P]_{\text {aq. }}} \\
(\mathrm{mg} / \mathrm{ml})\end{array}$ & $\begin{array}{c}{[P]_{r m+o f f}} \\
(\mathrm{mg} / \mathrm{ml})\end{array}$ \\
\hline 1 & $18.0(1.02)$ & 0.270 & 12.18 & 2.70 & 1.05 \\
5 & $4.2(0.08)$ & 0.056 & 2.52 & 0.56 & 2.81 \\
10 & $3.0(0.08)$ & 0.037 & 1.68 & 0.37 & 2.96 \\
20 & $1.9(0.08)$ & 0.020 & 0.91 & 0.20 & 3.10 \\
30 & $8.0(0.16)$ & 0.115 & 5.18 & 1.15 & 2.33 \\
50 & $15.9(0.40)$ & 0.238 & 10.71 & 2.38 & 1.32 \\
70 & $18.1(0.82)$ & 0.272 & 12.25 & 2.72 & 1.04 \\
\hline
\end{tabular}

* Standard deviation in ( ), at least 3 repeat tests. 


\section{2-9 Partition coefficient at infinite dilution for forward extraction, $K_{E . f w}^{\infty}$}

$K_{E . f w}^{\infty}$ is calculated when the distribution coefficient of forward extraction, $K_{E . f w}$, is plotted against the concentration of penicillin in the aqueous phase, $[P]_{a q . f . f w}$, (all other parameters being held constant) and the experimental data extrapolated back to $[P]_{\text {aqf.fw }}=0$.

a) $[S]=88 \mathrm{~g} / \mathrm{l}, \mathrm{pH} 7.6$

\begin{tabular}{|c|c|c|c|c|}
\hline$[P]_{\text {aq.i.jw }}(\mathrm{g} / \mathrm{l})$ & {$[P]_{\text {aq.ffw }}(\mathrm{g} / \mathrm{l})$} & {$[P]_{m+0 . f . j w}(\mathrm{~g} / \mathrm{l})$} & $K_{E . f w}$ & $K_{E . f w}^{\infty}$ \\
\hline 0.0 & 0.00 & 0.00 & & \\
1.8 & 0.34 & 1.45 & 4.27 & \\
3.6 & 0.68 & 2.90 & 4.27 & \\
7.1 & 1.32 & 5.74 & 4.36 & \\
10.7 & 2.01 & 8.62 & 4.29 & \\
14.3 & 2.67 & 11.54 & 4.31 & 4.26 \\
17.8 & 3.31 & 14.38 & 4.34 & \\
21.4 & 3.86 & 17.40 & 4.50 & \\
24.9 & 4.51 & 20.23 & 4.48 & \\
28.5 & 5.15 & 23.17 & 4.50 & \\
32.1 & 5.84 & 26.05 & 4.46 & \\
35.6 & 6.50 & 28.88 & 4.45 & \\
\hline
\end{tabular}


b) $[S]=267 \mathrm{~g} / 1$, pH 7.6

\begin{tabular}{|c|c|c|c|c|}
\hline$[P]_{\text {aq.ifw }}(\mathrm{g} / \mathrm{l})$ & {$[P]_{\text {aq.ffw }}(\mathrm{g} / \mathrm{l})$} & {$[P]_{m \mathrm{~m}+\mathrm{f} f \mathrm{fw}}(\mathrm{g} / \mathrm{l})$} & $K_{E, f w}$ & $K_{E . f w}^{\infty}$ \\
\hline 0.0 & 0.00 & 0.00 & & \\
1.8 & 0.24 & 1.44 & 5.96 & \\
3.6 & 0.50 & 2.87 & 5.75 & \\
7.1 & 0.97 & 5.66 & 5.87 & \\
10.7 & 1.40 & 8.58 & 6.12 & \\
14.3 & 1.93 & 11.42 & 5.92 & 5.8 \\
17.8 & 2.40 & 14.22 & 5.93 & \\
21.4 & 2.85 & 17.12 & 6.01 & \\
24.9 & 3.24 & 19.99 & 6.17 & \\
28.5 & 3.74 & 22.85 & 6.12 & \\
32.1 & 4.19 & 25.76 & 6.15 & \\
35.6 & 4.64 & 28.57 & 6.16 & \\
\hline
\end{tabular}

c) $[S]=445 \mathrm{~g} / 1, \mathrm{pH} 7.6$

\begin{tabular}{|c|c|c|c|c|}
\hline$[P]_{a q . i f w}(\mathrm{~g} / \mathrm{l})$ & {$[P]_{\text {aqf.f.j }}(\mathrm{g} / \mathrm{l})$} & {$[P]_{m++o f f w}(\mathrm{~g} / \mathrm{l})$} & $K_{E, f w}$ & $K_{E . f w}^{\infty}$ \\
\hline 0.0 & 0.00 & 0.00 & & \\
1.8 & 0.19 & 1.21 & 6.45 & \\
3.6 & 0.40 & 2.40 & 6.05 & \\
7.1 & 0.77 & 4.74 & 6.15 & \\
10.7 & 1.14 & 7.15 & 6.25 & \\
14.3 & 1.49 & 9.57 & 6.41 & 6.2 \\
17.8 & 1.94 & 11.88 & 6.13 & \\
21.4 & 2.19 & 14.35 & 6.54 & \\
24.9 & 2.66 & 16.64 & 6.25 & \\
28.5 & 3.08 & 19.04 & 6.18 & \\
32.1 & 3.46 & 21.45 & 6.21 & \\
35.6 & 3.71 & 23.84 & 6.42 & \\
\hline
\end{tabular}


d) $[S]=88 \mathrm{~g} / \mathrm{l}, \mathrm{pH} 1.65$

\begin{tabular}{|c|c|c|c|c|}
\hline$[P]_{\text {aq.i.fw }}(\mathrm{g} / \mathrm{l})$ & {$[P]_{\text {aq.ffw }}(\mathrm{g} / \mathrm{l})$} & {$[P]_{r m+o . f f w}(\mathrm{~g} / \mathrm{l})$} & $K_{E . f w}$ & $K_{E . f w}^{\infty}$ \\
\hline 0.0 & 0.00 & 0.00 & & \\
1.8 & 0.25 & 1.53 & 6.02 & 6.2 \\
3.6 & 0.50 & 3.08 & 6.22 & \\
7.1 & 0.96 & 6.09 & 6.32 & \\
10.7 & 1.56 & 9.07 & 5.83 & \\
\hline
\end{tabular}

e) $[S]=267 \mathrm{~g} / \mathrm{l}, \mathrm{pH} 1.65$

\begin{tabular}{|c|c|c|c|c|}
\hline$[P]_{\text {aq.ifw }}(\mathrm{g} / \mathrm{l})$ & {$[P]_{\text {aq.f.fw }}(\mathrm{g} / \mathrm{l})$} & {$[P]_{r m+o . f . j w}(\mathrm{~g} / \mathrm{l})$} & $K_{E . f w}$ & $K_{E . f w}^{\infty}$ \\
\hline 0.0 & 0.00 & 0.00 & & \\
1.8 & 0.20 & 1.47 & 7.27 & \\
3.6 & 0.42 & 2.93 & 6.97 & 7.3 \\
7.1 & 0.83 & 5.78 & 7.01 & \\
10.7 & 1.28 & 8.68 & 6.80 & \\
\hline
\end{tabular}

f) $[S]=445 \mathrm{~g} / \mathrm{l}, \mathrm{pH} 1.65$

\begin{tabular}{|c|c|c|c|c|}
\hline $\begin{array}{c}{[P]_{\text {ag. ifw }}} \\
(\mathrm{g} / \mathrm{l})\end{array}$ & $\begin{array}{c}{[P]_{\text {aq.f.fw }}} \\
(\mathrm{g} / \mathrm{l})\end{array}$ & $\begin{array}{c}{[P]_{r m+o f f f w}} \\
(\mathrm{~g} / \mathrm{l})\end{array}$ & $K_{E, f w}$ & $K_{E . f w}^{\infty}$ \\
\hline 0.0 & 0.00 & 0.00 & & \multirow{2}{*}{7.5} \\
\hline 1.8 & 0.16 & 1.22 & 7.44 \\
\hline 3.6 & 0.33 & 2.43 & 7.44 & \\
\hline 7.1 & 0.65 & 4.79 & 7.33 \\
\hline 10.7 & 1.00 & 7.21 & 7.18 & \\
\hline
\end{tabular}




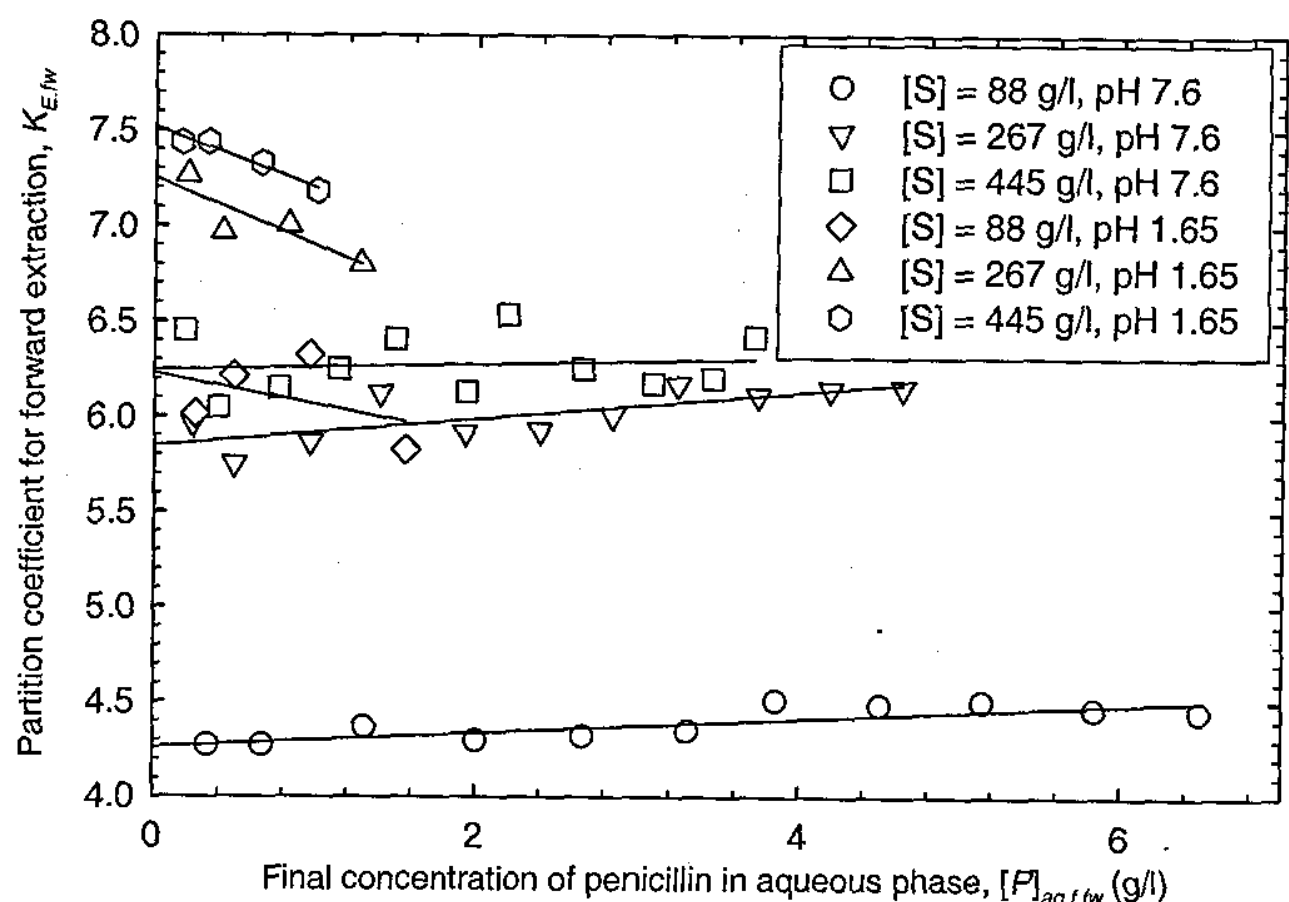

Figure A2-1: Determination of $K_{E . f w}^{\infty}$ using a plot of $K_{E, f^{\prime}}$ versus $[P]_{a q . f f w^{\prime}}$. 


\section{Appendix 3}

Experimental Results for Backward Extraction 


\section{3-1 Backward extraction time}

Parameters:

Density of water, $\rho_{w}=1.0 \mathrm{~g} / \mathrm{ml}$

Volume of titration for blank sample, $V_{\text {titrantblank }}=0.6 \mathrm{ml}$

Molarity of acid, $M_{a c i d}=0.05 \mathrm{~mol} / \mathrm{l}$

Initial concentration of penicillin $\mathrm{G}$ in the aqueous phase before forward extraction, $[P]_{\text {aq.i.f. } f w}=7.1 \mathrm{~g} / 1$

Final mass of penicillin $\mathrm{G}$ in the reverse micelles after forward extraction, $P_{r m+o f f f w}=$ $29 \mathrm{mg}\left(=P_{r m+o . i . b w}\right)$

Final volume of water in the aqueous phase, $V_{a q . f . b w}=6.0 \mathrm{ml}$

Initial volume of water in the aqueous phase, $V_{\text {aq.i.bw }}=5.0 \mathrm{ml}$

\begin{tabular}{|c|c|c|c|c|}
\hline $\begin{array}{c}\text { Time } \\
(\mathrm{min})\end{array}$ & $\begin{array}{c}V_{\text {titrant }}{ }^{*} \\
(\mathrm{ml})\end{array}$ & $\% N$ & $\begin{array}{c}P_{\text {aqf.sw }} \\
(\mathrm{mg})\end{array}$ & $\begin{array}{c}{[P]_{\text {aqf.j.b }}} \\
(\mathrm{mg} / \mathrm{ml})\end{array}$ \\
\hline 0 & $0.95(0.41)$ & 0.004 & 0.24 & 0.04 \\
1 & $6.50(0.08)$ & 0.068 & 4.13 & 0.68 \\
2 & $8.90(0.16)$ & 0.096 & 5.81 & 0.96 \\
3 & $11.70(0.24)$ & 0.129 & 7.77 & 1.29 \\
4 & $17.90(0.41)$ & 0.202 & 12.11 & 2.01 \\
5 & $17.80(0.41)$ & 0.200 & 12.04 & 2.00 \\
7 & $17.90(0.41)$ & 0.202 & 12.11 & 2.01 \\
10 & $18.00(0.41)$ & 0.203 & 12.18 & 2.03 \\
15 & $17.90(0.41)$ & 0.202 & 12.11 & 2.01 \\
\hline
\end{tabular}

* Standard deviation in ( ), at least 3 repeat tests. 


\section{3-2 Effect of settling time}

Parameters:

Density of water, $\rho_{w}=1.0 \mathrm{~g} / \mathrm{ml}$

Volume of titration for blank sample, $V_{\text {titrantblank }}=0.6 \mathrm{ml}$

Molarity of acid, $M_{\text {acid }}=0.05 \mathrm{~mol} / 1$

Initial concentration of penicillin $\mathrm{G}$ the in aqueous phase before forward extraction, $[P]_{\text {aq.i.f.fw }}=7.1 \mathrm{~g} / 1$

Final mass of penicillin $G$ in the reverse micelles after forward extraction, $P_{r m+o f f f w}=$ $29 \mathrm{mg}\left(=P_{r m+o . i . b w}\right)$

Final volume of water in the aqueous phase, $V_{a q f . b w}=6.0 \mathrm{ml}$

Initial volume of water in the aqueous phase, $V_{\text {aq.i.bw }}=5.0 \mathrm{ml}$

\begin{tabular}{|c|c|c|c|c|c|}
\hline $\begin{array}{c}\text { Time } \\
(\mathrm{min})\end{array}$ & $\begin{array}{c}V_{\text {aqf.bw }} \\
(\mathrm{ml})\end{array}$ & $\begin{array}{c}V_{\text {titrant }}{ }^{*} \\
(\mathrm{ml})\end{array}$ & $\% N$ & $\begin{array}{c}P_{\text {aqf.j.w }} \\
(\mathrm{mg})\end{array}$ & $\begin{array}{c}{[P]_{\text {aqf.bw }}} \\
(\mathrm{mg} / \mathrm{ml})\end{array}$ \\
\hline 1 & 5.5 & $6.50(0.08)$ & 0.075 & 4.13 & 0.75 \\
5 & 5.5 & $10.00(0.16)$ & 0.119 & 6.58 & 1.19 \\
10 & 6.0 & $18.00(0.41)$ & 0.203 & 12.18 & 2.03 \\
15 & 6.0 & $17.50(0.41)$ & 0.197 & 11.83 & 1.97 \\
20 & 6.0 & $17.50(0.41)$ & 0.197 & 11.83 & 1.97 \\
25 & 6.0 & $18.00(0.41)$ & 0.203 & 12.18 & 2.03 \\
\hline
\end{tabular}

* Standard deviation in ( ), at least 3 repeat tests. 


\section{3-3 Additional volume of backward extraction}

Parameter:

Initial volume of water in the aqueous phase, $V_{\text {aq.i.bw }}=5.0 \mathrm{ml}$

\begin{tabular}{|c|c|c|c|}
\hline $\begin{array}{c}{[S]} \\
(\mathrm{g} / \mathrm{l})\end{array}$ & $\begin{array}{c}\text { Additional volume. - pH 2 } \\
(\mathrm{ml})\end{array}$ & $\begin{array}{c}\text { Additional volume. } \mathrm{pH} 7^{*} \\
(\mathrm{ml})\end{array}$ & $\begin{array}{c}\text { Additional volume. }-\mathrm{pH} \mathrm{10*} \\
(\mathrm{ml})\end{array}$ \\
\hline 0 & $0.00(0.000)$ & $0.00(0.000)$ & $0.00(0.000)$ \\
88 & $0.45(0.008)$ & $0.50(0.008)$ & $0.50(0.008)$ \\
267 & $0.80(0.040)$ & $1.00(0.040)$ & $1.20(0.040)$ \\
333 & $1.15(0.082)$ & $1.30(0.040)$ & $1.50(0.040)$ \\
445 & $2.20(0.160)$ & $2.80(0.160)$ & $3.00(0.160)$ \\
\hline
\end{tabular}

* Standard deviation in ( ), at least 3 repeat tests. 


\section{3-4 Effect of salt concentration}

Parameters:

Density of water, $\rho_{w}=1.0 \mathrm{~g} / \mathrm{ml}$

Volume of titration for blank sample, $V_{\text {titrantblank }}=0.6 \mathrm{ml}$

Molarity of acid, $M_{\text {acid }}=0.05 \mathrm{~mol} / 1$

Initial concentration of penicillin $\mathrm{G}$ in the aqueous phase before forward extraction, $[P]_{\text {aq.i.f.fw }}=7.1 \mathrm{~g} / \mathrm{l}$

Final mass of penicillin $\mathrm{G}$ in reverse micelles after the forward extraction, $P_{r m+o f f w}=$ $29 \mathrm{mg}\left(=P_{r m+o . i . b w}\right)$

Final volume of water in the aqueous phase, $V_{a q . f . b w}=6.0 \mathrm{ml}$

Initial volume of water in the aqueous phase, $V_{\text {aq. } i . b w}=5.0 \mathrm{ml}$

\begin{tabular}{|c|c|c|c|c|}
\hline $\begin{array}{c}{[\mathrm{KCl}]} \\
(\mathrm{g} / \mathrm{l})\end{array}$ & $\begin{array}{c}V_{\text {titrant }}{ }^{*} \\
(\mathrm{ml})\end{array}$ & $\% \mathrm{~N}$ & $\begin{array}{c}P_{\text {aq.f.bw }} \\
(\mathrm{mg})\end{array}$ & $\begin{array}{c}{[P]_{\text {aq.f.bw }}} \\
(\mathrm{mg} / \mathrm{ml})\end{array}$ \\
\hline 0 & $0.60(0.00)$ & 0.000 & 0.000 & 0.000 \\
7 & $0.60(0.00)$ & 0.000 & 0.000 & 0.000 \\
15 & $4.70(0.16)$ & 0.048 & 2.872 & 0.479 \\
22 & $8.50(0.24)$ & 0.092 & 5.534 & 0.922 \\
30 & $10.80(0.41)$ & 0.119 & 7.145 & 1.191 \\
37 & $17.80(0.41)$ & 0.201 & 12.049 & 2.008 \\
75 & $17.90(0.41)$ & 0.202 & 12.119 & 2.020 \\
112 & $18.00(0.41)$ & 0.203 & 12.189 & 2.031 \\
149 & $17.90(0.41)$ & 0.202 & 12.119 & 2.020 \\
\hline
\end{tabular}

* Standard deviation in ( ), at least 3 repeat tests. 


\section{3-5 Effect of $\mathrm{pH}$}

a) $[S]=88 \mathrm{~g} h$

Parameters:

Density of water, $\rho_{w}=1.0 \mathrm{~g} / \mathrm{ml}$

Volume of titration for blank sample, $V_{\text {titrantblank }}=0.6 \mathrm{ml}$

Molarity of acid, $M_{\text {acid }}=0.05 \mathrm{~mol} / \mathrm{l}$

Initial concentration of penicillin $\mathrm{G}$ in the aqueous phase before forward extraction,

$[P]_{\text {aq.i.f. } f w}=7.1 \mathrm{~g} / 1$

Final mass of penicillin $G$ in the reverse micelles after the forward extraction,

$P_{r m+o . f . f w}=29 \mathrm{mg}\left(=P_{r m+o . i . b w}\right)$

Initial volume of water in the aqueous phase, $V_{\text {aq.i.bw }}=5.0 \mathrm{ml}$

\begin{tabular}{|c|c|c|c|c|c|}
\hline $\mathrm{pH}$ & $\begin{array}{c}V_{\text {aqfibw }} \\
(\mathrm{ml})\end{array}$ & $\begin{array}{c}\hat{V}_{\text {titant }}{ }^{*} \\
(\mathrm{ml})\end{array}$ & $\% N$ & $\begin{array}{c}P_{\text {aq.j.bw }} \\
(\mathrm{mg})\end{array}$ & $\begin{array}{c}{[P]_{a q . j \mathrm{fb}}} \\
(\mathrm{mg} / \mathrm{ml})\end{array}$ \\
\hline 2 & 5.35 & $13.50(0.82)$ & 0.169 & 9.04 & 1.69 \\
3 & 5.35 & $13.50(0.82)$ & 0.169 & 9.04 & 1.69 \\
4 & 5.40 & $13.50(0.82)$ & 0.167 & 9.04 & 1.67 \\
5 & 5.45 & $13.50(0.82)$ & 0.166 & 9.04 & 1.66 \\
6 & 5.50 & $13.50(0.82)$ & 0.164 & 9.04 & 1.64 \\
7 & 5.50 & $13.80(0.82)$ & 0.168 & 9.25 & 1.68 \\
8 & 5.50 & $13.80(0.82)$ & 0.168 & 9.25 & 1.68 \\
9 & 5.50 & $13.80(0.82)$ & 0.168 & 9.25 & 1.68 \\
10 & 5.50 & $13.80(0.82)$ & 0.168 & 9.25 & 1.68 \\
11 & 5.50 & $13.80(0.82)$ & 0.168 & 9.25 & 1.68 \\
\hline
\end{tabular}

* Standard deviation in ( ), at least 3 repeat tests.

b) $[S]=267 \mathrm{~g} / \mathrm{l}$

Parameters:

Density of water, $\rho_{w}=1.0 \mathrm{~g} / \mathrm{ml}$

Volume of titration for blank sample, $V_{\text {titrantblank }}=0.6 \mathrm{ml}$

Molarity of acid, $M_{\text {acid }}=0.05 \mathrm{~mol} / \mathrm{l}$ 
Initial concentration of penicillin $\mathrm{G}$ in the aqueous phase before forward extraction,

$[P]_{\text {aq.if. } f \mathrm{w}}=7.1 \mathrm{~g} / 1$

Final mass of penicillin $\mathrm{G}$ in the reverse micelles after forward extraction, $P_{r m+o . f . f w}=$ $29 \mathrm{mg}\left(=P_{r m+o . i . b w}\right)$

Initial volume of water in the aqueous phase, $V_{a q . i . b w}=5.0 \mathrm{ml}$

\begin{tabular}{|c|c|c|c|c|c|}
\hline $\mathrm{pH}$ & $\begin{array}{c}V_{\text {aq.f.bw }} \\
(\mathrm{ml})\end{array}$ & $\begin{array}{c}V_{\text {titrant }}{ }^{*} \\
(\mathrm{ml})\end{array}$ & $\% \mathrm{~N}$ & $\begin{array}{c}P_{\text {aq.f.bw }} \\
(\mathrm{mg})\end{array}$ & $\begin{array}{c}{[P]_{a q . f b w}} \\
(\mathrm{mg} / \mathrm{ml})\end{array}$ \\
\hline 2 & 5.80 & $20.00(0.82)$ & 0.234 & 13.59 & 2.34 \\
3 & 5.80 & $20.00(0.82)$ & 0.234 & 13.59 & 2.34 \\
4 & 5.85 & $20.00(0.82)$ & 0.232 & 13.59 & 2.32 \\
5 & 5.90 & $20.50(0.82)$ & 0.236 & 13.94 & 2.36 \\
6 & 5.95 & $20.50(0.82)$ & 0.234 & 13.94 & 2.34 \\
7 & 6.00 & $20.00(0.82)$ & 0.226 & 13.59 & 2.26 \\
8 & 6.00 & $20.00(0.82)$ & 0.226 & 13.59 & 2.26 \\
9 & 6.10 & $20.00(0.82)$ & 0.223 & 13.59 & 2.23 \\
10 & 6.20 & $20.50(0.82)$ & 0.225 & 13.94 & 2.25 \\
11 & 6.20 & $20.50(0.82)$ & 0.225 & 13.94 & 2.25 \\
\hline
\end{tabular}

* Standard deviation in ( ), at least 3 repeat tests.

c) $[S]=445 \mathrm{~g} / \mathrm{l}$

Parameters:

Density of water, $\rho_{w}=1.0 \mathrm{~g} / \mathrm{ml}$

Volume of titration for blank sample, $V_{\text {titrantblank }}=0.6 \mathrm{ml}$

Molarity of acid, $M_{\text {acid }}=0.05 \mathrm{~mol} / \mathrm{l}$

Initial concentration of penicillin $\mathrm{G}$ in the aqueous phase before forward extraction, $[P]_{\text {aq.i.f. } f w}=7.1 \mathrm{~g} / \mathrm{l}$

Final mass of penicillin $\mathrm{G}$ in the reverse micelles after forward extraction, $P_{r m+o f f . f w}=$ $29 \mathrm{mg}\left(=P_{r m+o . i . b w}\right)$

Initial volume of water in the aqueous phase, $V_{a q . i . b w}=5.0 \mathrm{ml}$ 


\begin{tabular}{|c|c|c|c|c|c|}
\hline $\mathrm{pH}$ & $\begin{array}{c}V_{\text {aqf.fbw }} \\
(\mathrm{ml})\end{array}$ & $\begin{array}{c}V_{\text {titrant }}{ }^{*} \\
(\mathrm{ml})\end{array}$ & $\% N$ & $\begin{array}{c}P_{\text {aq.j.bw }} \\
(\mathrm{mg})\end{array}$ & $\begin{array}{c}{[P]_{\text {aqf. } . b w}} \\
(\mathrm{mg} / \mathrm{ml})\end{array}$ \\
\hline 2 & 6.70 & $22.00(1.02)$ & 0.224 & 14.99 & 2.24 \\
3 & 6.70 & $23.00(1.02)$ & 0.234 & 15.69 & 2.34 \\
4 & 6.70 & $23.00(1.02)$ & 0.234 & 15.69 & 2.34 \\
5 & 6.75 & $23.00(1.02)$ & 0.232 & 15.69 & 2.32 \\
6 & 6.75 & $23.00(1.02)$ & 0.232 & 15.69 & 2.32 \\
7 & 6.75 & $23.00(1.02)$ & 0.232 & 15.69 & 2.32 \\
8 & 6.75 & $22.00(1.02)$ & 0.222 & 14.99 & 2.22 \\
9 & 7.00 & $22.00(1.02)$ & 0.214 & 14.99 & 2.14 \\
10 & 7.00 & $22.00(1.02)$ & 0.214 & 14.99 & 2.14 \\
11 & 7.00 & $22.00(1.02)$ & 0.214 & 14.99 & 2.14 \\
\hline
\end{tabular}

* Standard deviation in ( ), at least 3 repeat tests. 


\section{3-6 Effect of initial mass of penicillin $G$ before forward extraction and} surfactant concentration

a) $[S]=88 \mathrm{~g} / \mathrm{l}, \underline{\mathrm{pH}}=7.6$

\section{Parameters:}

Density of water, $\rho_{w}=1.0 \mathrm{~g} / \mathrm{ml}$

Volume of titration for blank sample, $V_{\text {titrantblank }}=0.6 \mathrm{ml}$

Molarity of acid, $M_{\text {acid }}=0.05 \mathrm{~mol} / \mathrm{l}$

Initial volume of water in the backward aqueous phase, $V_{a q . i b w}=5.0 \mathrm{ml}$

Final volume of water in the backward aqueous phase, $V_{a q . f b w}=5.5 \mathrm{ml}$

*Final mass of penicillin $\mathrm{G}$ in the reverse micelles after forward extraction, $P_{r m+o \text {.f.f }}$ is obtained from the results in Appendix A2-1

\begin{tabular}{|c|c|c|c|c|c|}
\hline $\begin{array}{c}{[P]_{\text {aq.ifw }}} \\
(\mathrm{mg} / \mathrm{ml})\end{array}$ & $\begin{array}{c}{[P]_{r m+o f f f w}} \\
(\mathrm{mg} / \mathrm{ml})\end{array}$ & $\begin{array}{c}V_{\text {titrant }}{ }^{*} \\
(\mathrm{ml})\end{array}$ & $\% N$ & $\begin{array}{c}P_{\text {ag.f.bw }} \\
(\mathrm{mg})\end{array}$ & $\begin{array}{c}{[P]_{\text {aqf.jow }}} \\
(\mathrm{mg} / \mathrm{ml})\end{array}$ \\
\hline 0 & 0.00 & $0.60(0.00)$ & 0.000 & 0.00 & 0.00 \\
3.6 & 2.90 & $11.00(0.24)$ & 0.132 & 7.29 & 1.32 \\
7.1 & 5.74 & $13.80(0.24)$ & 0.168 & 9.25 & 1.68 \\
10.7 & 8.62 & $15.70(0.32)$ & 0.192 & 10.58 & 1.92 \\
14.3 & 11.54 & $18.30(0.41)$ & 0.225 & 12.40 & 2.25 \\
17.8 & 14.38 & $20.00(0.41)$ & 0.247 & 13.59 & 2.47 \\
21.4 & 17.40 & $22.10(0.49)$ & 0.274 & 15.06 & 2.74 \\
24.9 & 20.23 & $22.00(0.49)$ & 0.273 & 14.99 & 2.73 \\
28.5 & 23.17 & $22.00(0.49)$ & 0.273 & 14.99 & 2.73 \\
32.1 & 26.05 & $22.05(0.49)$ & 0.273 & 15.03 & 2.73 \\
35.6 & 28.88 & $22.10(0.49)$ & 0.274 & 15.06 & 2.74 \\
\hline
\end{tabular}

* Standard deviation in ( ), at least 3 repeat tests.

b) $[S]=267 \mathrm{~g} / \mathrm{l}, \mathrm{pH} 7.6$

Parameters:

Density of water, $\rho_{w}=1.0 \mathrm{~g} / \mathrm{ml}$

Volume of titration for blank sample, $V_{\text {titrantblank }}=0.6 \mathrm{ml}$

Molarity of acid, $M_{\text {acid }}=0.05 \mathrm{~mol} / 1$

Initial volume of water in the backward aqueous phase, $V_{\text {aq.i.bw }}=5.0 \mathrm{ml}$ 
Final volume of water in the backward aqueous phase, $V_{a q . f . b w}=6.0 \mathrm{ml}$

*Final mass of penicillin $\mathrm{G}$ in the reverse micelles after forward extraction, $P_{r m+o f f w}$ is obtained from the results in Appendix A2-1

\begin{tabular}{|c|c|c|c|c|c|}
\hline $\begin{array}{c}{[P]_{\text {aq.ifw }}} \\
(\mathrm{mg} / \mathrm{ml})\end{array}$ & $\begin{array}{c}{[P]_{r m+o . f f w}} \\
(\mathrm{mg} / \mathrm{ml})\end{array}$ & $\begin{array}{c}V_{\text {titrant }}{ } \\
(\mathrm{ml})\end{array}$ & $\% N$ & $\begin{array}{c}P_{\text {aqf.j.w }} \\
(\mathrm{mg})\end{array}$ & $\begin{array}{c}{[P]_{\text {aq.j.bw }}} \\
(\mathrm{mg} / \mathrm{ml})\end{array}$ \\
\hline 0 & 0.00 & $0.60(0.00)$ & 0.000 & 0.00 & 0.00 \\
3.6 & 2.87 & $15.00(0.32)$ & 0.168 & 10.09 & 1.68 \\
7.1 & 5.66 & $20.10(0.49)$ & 0.228 & 13.66 & 2.28 \\
10.7 & 8.58 & $22.50(0.49)$ & 0.256 & 15.34 & 2.56 \\
14.3 & 11.42 & $22.50(0.49)$ & 0.256 & 15.34 & 2.56 \\
17.8 & 14.22 & $27.00(0.57)$ & 0.308 & 18.49 & 3.08 \\
21.4 & 17.12 & $30.00(0.65)$ & 0.343 & 20.59 & 3.43 \\
24.9 & 19.99 & $30.20(0.65)$ & 0.346 & 20.73 & 3.46 \\
28.5 & 22.85 & $30.10(0.65)$ & 0.344 & 20.66 & 3.44 \\
32.1 & 25.76 & $30.10(0.65)$ & 0.344 & 20.66 & 3.44 \\
35.6 & 28.57 & $30.10(0.65)$ & 0.344 & 20.66 & 3.44 \\
\hline
\end{tabular}

* Standard deviation in ( ), at least 3 repeat tests.

\section{c) $[S]=445 \mathrm{~g} / 1, \mathrm{pH} 7.6$}

Parameters:

Density of water, $\rho_{w}=1.0 \mathrm{~g} / \mathrm{ml}$

Volume of titration for blank sample, $V_{\text {titrantblank }}=0.6 \mathrm{ml}$

Molarity of acid, $M_{\text {acid }}=0.05 \mathrm{~mol} / \mathrm{l}$

Initial volume of water in the backward aqueous phase, $V_{\text {aq.i.bw }}=5.0 \mathrm{ml}$

Final volume of water in the backward aqueous phase, $V_{a q . f . b w}=6.8 \mathrm{ml}$

*Final mass of penicillin $\mathrm{G}$ in the reverse micelles after forward extraction, $P_{r m+o \text {.f. } f w}$ is obtained from the results in Appendix A2-1 


\begin{tabular}{|c|c|c|c|c|c|}
\hline $\begin{array}{c}{[P]_{\text {aq. if. }}} \\
(\mathrm{mg} / \mathrm{ml})\end{array}$ & $\begin{array}{c}{[P]_{\text {rm +o.f.fw }}} \\
(\mathrm{mg} / \mathrm{ml})\end{array}$ & $\begin{array}{c}V_{\text {titrant }}{ }^{*} \\
(\mathrm{ml})\end{array}$ & $\% N$ & $\begin{array}{c}P_{\text {aq.f.bw }} \\
(\mathrm{mg})\end{array}$ & $\begin{array}{c}{[P]_{\text {aqf.j.bw }}} \\
(\mathrm{mg} / \mathrm{ml})\end{array}$ \\
\hline 0 & 0.00 & $0.60(0.00)$ & 0.000 & 0.00 & 0.00 \\
3.6 & 2.40 & $16.00(0.41)$ & 0.159 & 10.79 & 1.59 \\
7.1 & 4.74 & $23.00(0.49)$ & 0.231 & 15.69 & 2.31 \\
10.7 & 7.15 & $25.50(0.57)$ & 0.257 & 17.44 & 2.57 \\
14.3 & 9.57 & $27.50(0.57)$ & 0.277 & 18.84 & 2.77 \\
17.8 & 11.88 & $28.00(0.65)$ & 0.282 & 19.19 & 2.82 \\
21.4 & 14.35 & $30.50(0.73)$ & 0.308 & 20.94 & 3.08 \\
24.9 & 16.64 & $31.50(0.73)$ & 0.318 & 21.65 & 3.18 \\
28.5 & 19.04 & $31.50(0.73)$ & 0.318 & 21.65 & 3.18 \\
32.1 & 21.45 & $31.50(0.73)$ & 0.318 & 21.65 & 3.18 \\
35.6 & 23.84 & $31.50(0.73)$ & 0.318 & 21.65 & 3.18 \\
\hline
\end{tabular}

* Standard deviation in ( ), at least 3 repeat tests.

d) $[S]=88 \mathrm{~g} / 1, \mathrm{pH} 1.65$

Parameters:

Density of water, $\rho_{w}=1.0 \mathrm{~g} / \mathrm{ml}$

Volume of titration for blank sample, $V_{\text {titrantblank }}=0.6 \mathrm{ml}$

Molarity of acid, $M_{a c i d}=0.05 \mathrm{~mol} / \mathrm{l}$

Initial volume of water in the backward aqueous phase, $V_{a q . i b w}=5.0 \mathrm{ml}$

Final volume of water in the backward aqueous phase, $V_{a q . f . b w}=5.8 \mathrm{ml}$

*Final mass of penicillin $\mathrm{G}$ in the reverse micelles after forward extraction, $P_{r m+o f f f w}$ is obtained from the results in Appendix A2-1

\begin{tabular}{|c|c|c|c|c|}
\hline$[P]_{\text {aq.ifw }}(\mathrm{mg} / \mathrm{ml})$ & $\begin{array}{c}{[P]_{m m+o f f . w}} \\
(\mathrm{mg} / \mathrm{ml})\end{array}$ & $\begin{array}{c}V_{\text {tirrant }}{ } \\
(\mathrm{ml})\end{array}$ & $\begin{array}{c}P_{\text {aqf.bw }} \\
(\mathrm{mg})\end{array}$ & $\begin{array}{c}{[P]_{\text {aq.f.bw }}} \\
(\mathrm{mg} / \mathrm{ml})\end{array}$ \\
\hline 0 & 0.00 & $0.60(0.00)$ & 0.00 & 0.00 \\
3.6 & 3.08 & $10.50(0.24)$ & 6.93 & 1.30 \\
7.1 & 6.09 & $13.50(0.32)$ & 9.04 & 1.69 \\
10.7 & 9.07 & $14.00(0.32)$ & 9.39 & 1.75 \\
\hline
\end{tabular}

* Standard deviation in ( ), at least 3 repeat tests. 


\section{e) $[S]=267 \mathrm{~g} / \mathrm{l}, \mathrm{pH} 1.65$}

Parameters:

Density of water, $\rho_{w}=1.0 \mathrm{~g} / \mathrm{ml}$

Volume of titration for blank sample, $V_{\text {titrantblank }}=0.6 \mathrm{ml}$

Molarity of acid, $M_{\text {acid }}=0.05 \mathrm{~mol} / \mathrm{l}$

Initial volume of water in the backward aqueous phase, $V_{\text {aq.i.bw }}=5.0 \mathrm{ml}$

Final volume of water in the backward aqueous phase, $V_{a q . f . b w}=5.35 \mathrm{ml}$

* Final mass of penicillin $\mathrm{G}$ in the reverse micelles after forward extraction, $P_{r m+o f f f w}$ is obtained from the results in Appendix A2-1

\begin{tabular}{|c|c|c|c|c|c|}
\hline $\begin{array}{c}{[P]_{\text {aq.i.fw }}} \\
(\mathrm{mg} / \mathrm{ml})\end{array}$ & $\begin{array}{c}{[P]_{m m+o f f . w}} \\
(\mathrm{mg} / \mathrm{ml})\end{array}$ & $\begin{array}{c}V_{\text {titrant }}{ }^{*} \\
(\mathrm{ml})\end{array}$ & $\% N$ & $\begin{array}{c}P_{\text {aqf.j.w }} \\
(\mathrm{mg})\end{array}$ & $\begin{array}{c}{[P]_{\text {aq.f.bw }}} \\
(\mathrm{mg} / \mathrm{ml})\end{array}$ \\
\hline 0 & 0.00 & $0.60(0.00)$ & 0.000 & 0.00 & 0.00 \\
3.6 & 2.93 & $15.00(0.41)$ & 0.174 & 10.09 & 1.74 \\
7.1 & 5.78 & $20.00(0.49)$ & 0.234 & 13.59 & 2.34 \\
10.7 & 8.68 & $22.00(0.49)$ & 0.258 & 14.99 & 2.58 \\
\hline
\end{tabular}

* Standard deviation in ( ), at least 3 repeat tests.

\section{f) $[S]=445 \mathrm{~g} / \mathrm{l}, \mathrm{pH} 1.65$}

\section{Parameters:}

Density of water, $\rho_{w}=1.0 \mathrm{~g} / \mathrm{ml}$

Volume of titration for blank sample, $V_{\text {titrantblank }}=0.6 \mathrm{ml}$

Molarity of acid, $M_{\text {acid }}=0.05 \mathrm{~mol} / \mathrm{l}$

Initial volume of water in the backward aqueous phase, $V_{\text {aq.i.bw }}=5.0 \mathrm{ml}$

Final volume of water in the backward aqueous phase, $V_{a q . f . b w}=6.7 \mathrm{ml}$

*Final mass of penicillin $\mathrm{G}$ in the reverse micelles after forward extraction, $P_{r m+o f f f w}$ is obtained from the results in Appendix A2-1 


\begin{tabular}{|c|c|c|c|c|c|}
\hline $\begin{array}{c}{[P]_{\text {aq.i.fw }}} \\
(\mathrm{mg} / \mathrm{ml})\end{array}$ & $\begin{array}{c}{[P]_{m+o f f f w}} \\
(\mathrm{mg} / \mathrm{ml})\end{array}$ & $\begin{array}{c}V_{\text {titrant }}{ }^{*} \\
(\mathrm{mi})\end{array}$ & $\% N$ & $\begin{array}{c}P_{\text {aq.f.bw }} \\
(\mathrm{mg})\end{array}$ & $\begin{array}{c}{[P]_{\text {aq.f.bw }}} \\
(\mathrm{mg} / \mathrm{ml})\end{array}$ \\
\hline 0 & 0.00 & $0.60(0.00)$ & 0.000 & 0.00 & 0.00 \\
3.6 & 2.43 & $14.50(0.32)$ & 0.145 & 9.74 & 1.45 \\
7.1 & 4.79 & $22.00(0.49)$ & 0.224 & 14.99 & 2.24 \\
10.7 & 7.21 & $25.00(0.49)$ & 0.255 & 17.09 & 2.55 \\
\hline
\end{tabular}

* Standard deviation in ( ), at least 3 repeat tests.

\section{3-7 Removal efficiency}

a) $[S]=88 \mathrm{~g} / \mathrm{l}, \mathrm{pH}=7.6$

\begin{tabular}{|c|c|c|c|c|}
\hline $\begin{array}{c}{[P]_{\text {aq.ifw }}} \\
(\mathrm{mg} / \mathrm{ml})\end{array}$ & $\begin{array}{c}P_{\text {aq.ifw }} \\
(\mathrm{mg})\end{array}$ & $\begin{array}{c}P_{\text {orffow }} \\
(\mathrm{mg})\end{array}$ & $\begin{array}{c}P_{\text {aq. } f \mathrm{bw}} \\
(\mathrm{mg})\end{array}$ & $\begin{array}{c}\text { Removal } \\
\text { efficiency }(\%)\end{array}$ \\
\hline 0 & 0.00 & 0.00 & 0.00 & - \\
3.6 & 17.82 & 14.64 & 7.29 & 40.9 \\
7.1 & 35.64 & 28.99 & 9.25 & 25.9 \\
10.7 & 53.46 & 43.55 & 10.58 & 19.8 \\
14.3 & 71.27 & 58.26 & 12.40 & 17.4 \\
17.8 & 89.09 & 72.61 & 13.59 & 15.3 \\
21.4 & 106.91 & 87.88 & 15.06 & 14.1 \\
24.9 & 124.73 & 102.15 & 14.99 & 12.0 \\
28.5 & 142.55 & 117.00 & 14.99 & 10.5 \\
32.1 & 160.37 & 131.57 & 15.03 & 9.4 \\
35.6 & 178.19 & 145.85 & 15.06 & 8.5 \\
\hline
\end{tabular}


b) $[S]=267 \mathrm{~g} / \mathrm{l}, \mathrm{pH}=7.6$

\begin{tabular}{|c|c|c|c|c|}
\hline $\begin{array}{c}{[P]_{\text {aq.i.if }}} \\
(\mathrm{mg} / \mathrm{ml})\end{array}$ & $\begin{array}{c}P_{\text {aq.ifw }} \\
(\mathrm{mg})\end{array}$ & $\begin{array}{c}P_{\text {or.f.fw }} \\
(\mathrm{mg})\end{array}$ & $\begin{array}{c}P_{\text {aq.f.bw }} \\
(\mathrm{mg})\end{array}$ & $\begin{array}{c}\text { Removal } \\
\text { efficiency }(\%)\end{array}$ \\
\hline 0 & 0.00 & 0.00 & 0.00 & - \\
3.6 & 17.82 & 15.76 & 10.09 & 56.6 \\
7.1 & 35.64 & 31.16 & 13.66 & 38.3 \\
10.7 & 53.46 & 47.20 & 15.34 & 28.7 \\
14.3 & 71.27 & 62.81 & 15.34 & 21.5 \\
17.8 & 89.09 & 78.21 & 18.49 & 20.8 \\
21.4 & 106.91 & 94.18 & 20.59 & 19.3 \\
24.9 & 124.73 & 109.93 & 20.73 & 16.6 \\
28.5 & 142.55 & 125.69 & 20.66 & 14.5 \\
32.1 & 160.37 & 141.66 & 20.66 & 12.9 \\
35.6 & 178.19 & 157.13 & 20.66 & 11.6 \\
\hline
\end{tabular}

c) $[S]=445 \mathrm{~g} / \mathrm{l}, \mathrm{pH}=7.6$

\begin{tabular}{|c|c|c|c|c|}
\hline $\begin{array}{c}{[P]_{\text {aq.ifw }}} \\
(\mathrm{mg} / \mathrm{ml})\end{array}$ & $\begin{array}{c}P_{\text {aq.ifw }} \\
(\mathrm{mg})\end{array}$ & $\begin{array}{c}P_{\text {or. f.fw }} \\
(\mathrm{mg})\end{array}$ & $\begin{array}{c}P_{\text {aq.f.jw }} \\
(\mathrm{mg})\end{array}$ & $\begin{array}{c}\text { Removal } \\
\text { efficiency }(\%)\end{array}$ \\
\hline 0 & 0.00 & 0.00 & 0.00 & \\
3.6 & 17.82 & 16.81 & 10.79 & 60.5 \\
7.1 & 35.64 & 33.19 & 15.69 & 44.0 \\
10.7 & 53.46 & 50.07 & 17.44 & 32.6 \\
14.3 & 71.27 & 67.02 & 18.84 & 26.4 \\
17.8 & 89.09 & 83.19 & 19.19 & 21.5 \\
21.4 & 106.91 & 100.42 & 20.94 & 19.6 \\
24.9 & 124.73 & 116.51 & 21.65 & 17.4 \\
28.5 & 142.55 & 133.25 & 21.65 & 15.2 \\
32.1 & 160.37 & 150.13 & 21.65 & 13.5 \\
35.6 & 178.19 & 166.86 & 21.65 & 12.1 \\
\hline
\end{tabular}




\section{Appendix 4}

Thermodynamic Framework for Forward Extraction 


\section{4-1 Isotherm of penicillin $G$ for forward extraction}

a) $\mathrm{pH} 7.6$

Table A4-1: Isotherms of penicillin $\mathrm{G}$ at $\mathrm{pH} 7.6$.

\begin{tabular}{|c|c|c|c|c|c|}
\hline \multicolumn{2}{|c|}{$[S]=88(\mathrm{~g} / \mathrm{l})$} & \multicolumn{2}{|c|}{$[S]=267(\mathrm{~g} / \mathrm{l})$} & \multicolumn{2}{|c|}{$[S]=445(\mathrm{~g} / \mathrm{l})$} \\
\hline$[P]_{a q f f f w}(\mathrm{~g} / \mathrm{l})$ & {$[P]_{r m+0 . f f w}(\mathrm{~g} / \mathrm{l})$} & {$[P]_{a q, f f w}(\mathrm{~g} / \mathrm{l})$} & {$[P]_{r m+a . f f w}(\mathrm{~g} / \mathrm{l})$} & {$[P]_{a q, f . f w}(\mathrm{~g} / \mathrm{l})$} & {$[P]_{m+a f f . f w}(\mathrm{~g} / \mathrm{l})$} \\
\hline 0.00 & 0.00 & 0.00 & 0.00 & 0.00 & 0.00 \\
\hline 0.34 & 1.45 & 0.24 & 1.44 & 0.19 & 1.21 \\
\hline 0.68 & 2.90 & 0.50 & 2.87 & 0.40 & 2.40 \\
\hline 1.32 & 5.74 & 0.97 & 5.66 & 0.77 & 4.74 \\
\hline 2.01 & 8.62 & 1.40 & 8.58 & 1.14 & 7.15 \\
\hline 2.67 & 11.54 & 1.93 & 11.42 & 1.49 & 9.57 \\
\hline 3.31 & 14.38 & 2.40 & 14.22 & 1.94 & 11.88 \\
\hline 3.86 & 17.40 & 2.85 & 17.12 & 2.19 & 14.35 \\
\hline 4.51 & 20.23 & 3.24 & 19.99 & 2.66 & 16.64 \\
\hline 5.15 & 23.17 & 3.74 & 22.85 & 3.08 & 19.04 \\
\hline 5.84 & 26.05 & 4.19 & 25.76 & 3.46 & 21.45 \\
\hline 6.50 & 28.88 & 4.64 & 28.57 & 3.71 & 23.84 \\
\hline
\end{tabular}

b) $\mathrm{pH} 1.65$

Table A4-2: Isotherms of penicillin $\mathrm{G}$ at $\mathrm{pH} 1.65$.

\begin{tabular}{|c|c|c|c|c|c|}
\hline \multicolumn{2}{|c|}{$[S]=88(\mathrm{~g} / \mathrm{l})$} & \multicolumn{2}{|c|}{$[S]=267(\mathrm{~g} / \mathrm{l})$} & \multicolumn{2}{|c|}{$[S]=445(\mathrm{~g} / \mathrm{l})$} \\
\hline$[P]_{a q f f, w}(\mathrm{~g} / \mathrm{l})$ & {$[P]_{r m+o f f w w}(\mathrm{~g} / \mathrm{l})$} & {$[P]_{\text {aq,ffw }}(\mathrm{g} / \mathrm{l})$} & {$[P]_{r m+o f, f w}(\mathrm{~g} / \mathrm{l})$} & {$[P]_{a q, f f w}(\mathrm{~g} / \mathrm{l})$} & {$[P]_{r m+o, f, f, f w}(\mathrm{~g} / \mathrm{l})$} \\
\hline 0.00 & 0.00 & 0.00 & 0.00 & 0.00 & 0.00 \\
\hline 0.25 & 1.53 & 0.20 & 1.47 & 0.16 & 1.22 \\
\hline 0.50 & 3.08 & 0.42 & 2.93 & 0.33 & 2.43 \\
\hline 0.96 & 6.09 & 0.83 & 5.78 & 0.65 & 4.79 \\
\hline 1.56 & 9.07 & 1.28 & 8.68 & 1.00 & 7.21 \\
\hline
\end{tabular}




\section{4-2 Langmuir model fitted to experimental data}

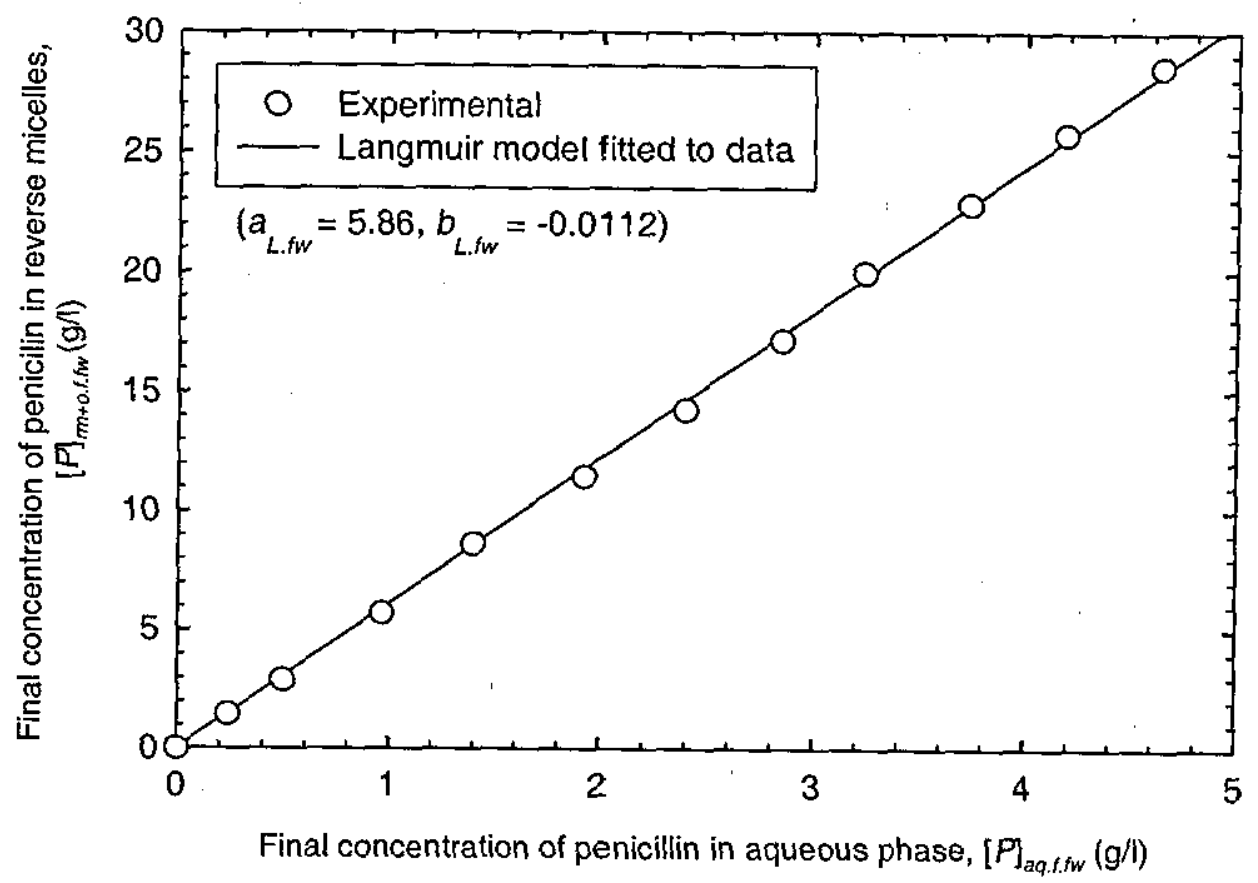

Figure A4.2-1: Langmuir model fitted to experimental data at $\mathrm{pH} 7.6$ and $[S]=267$ $\mathrm{g} / \mathrm{l}$.

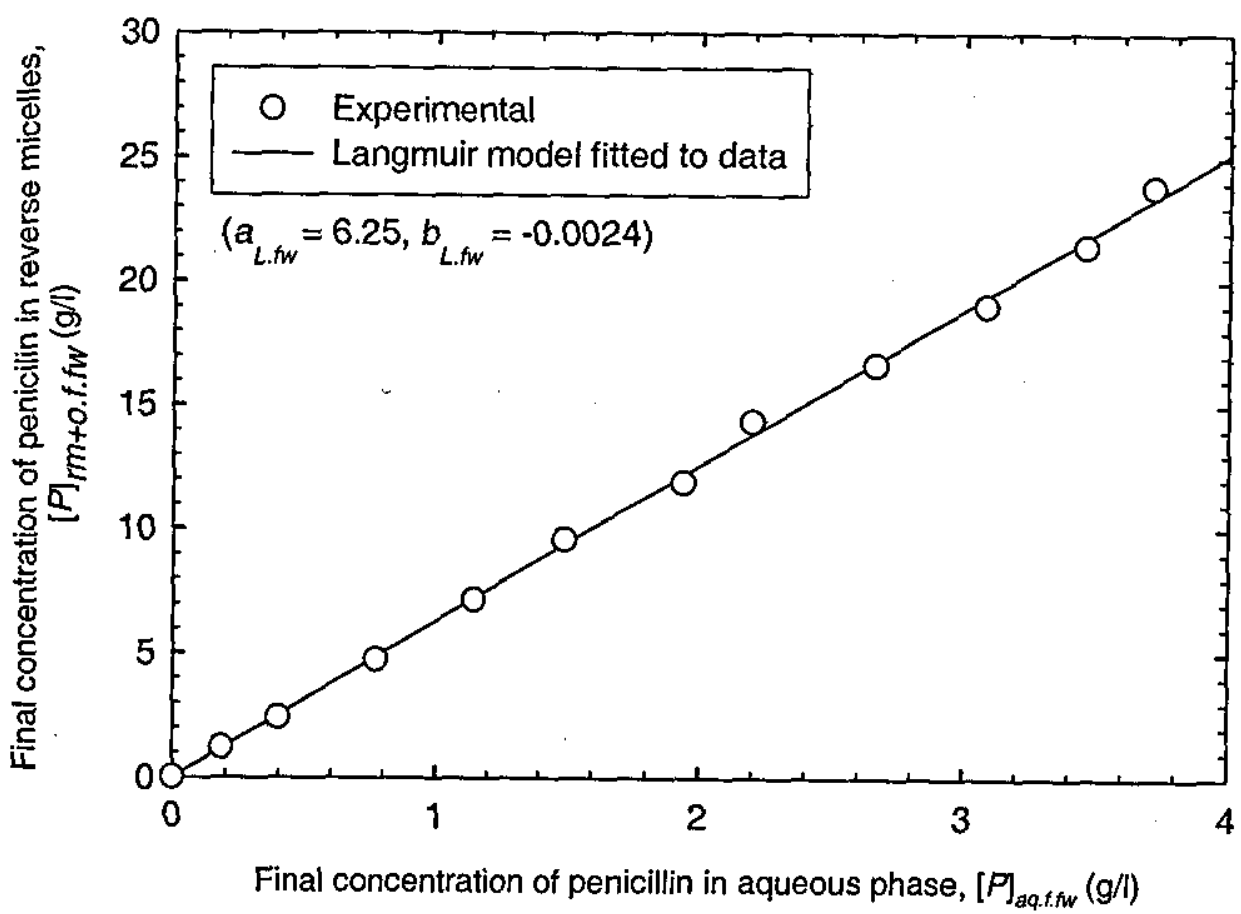

Figure A4.2-2: Langmuir model fitted to experimental data at $\mathrm{pH} 7.6$ and $[S]=445$ $\mathrm{g} / \mathrm{l}$. 


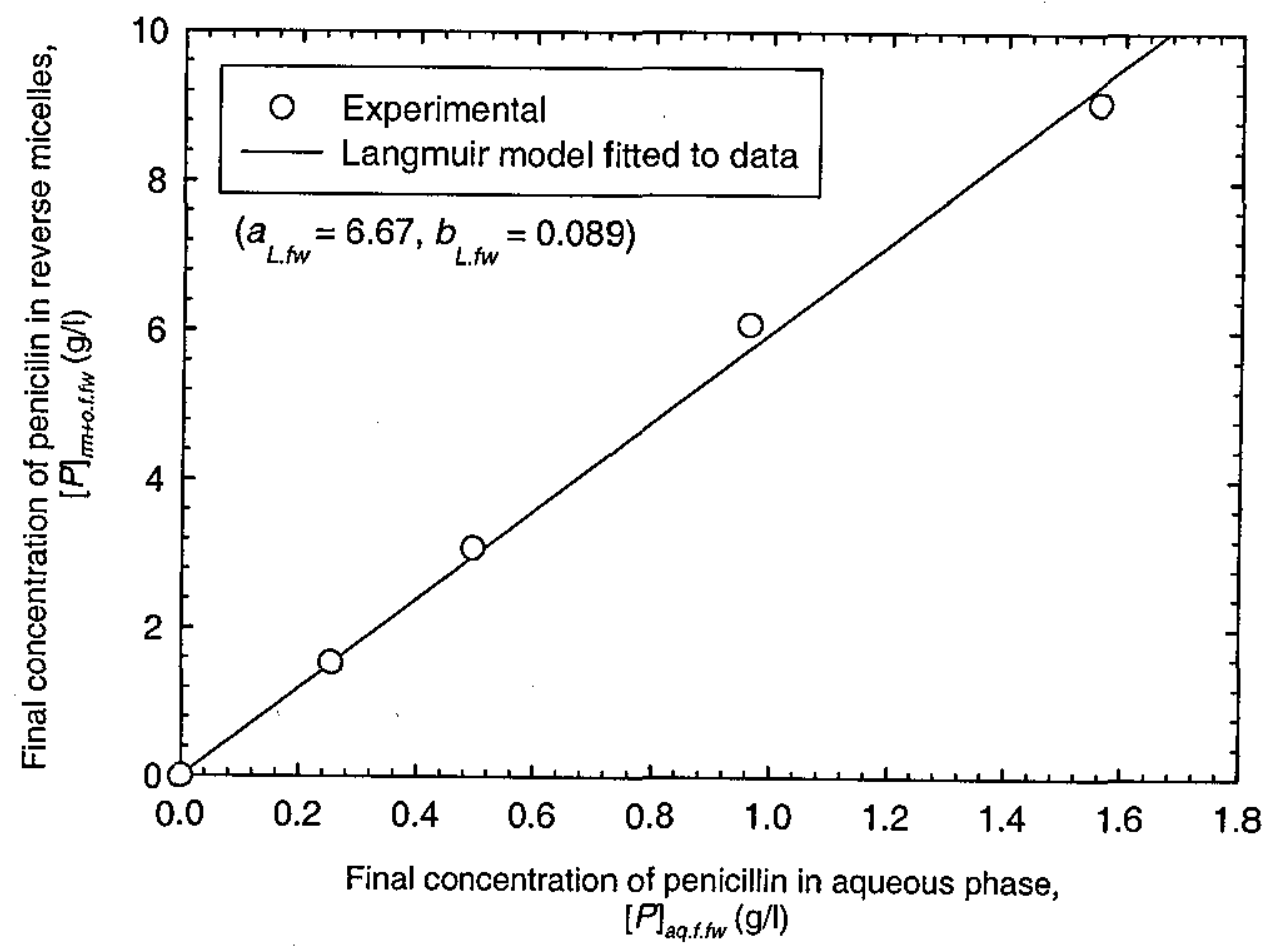

Figure A4.2-3: Langmuir model fitted to experimental data at $\mathrm{pH} 1.65$ and $[S]=88$ $\mathrm{g} / \mathrm{l}$.

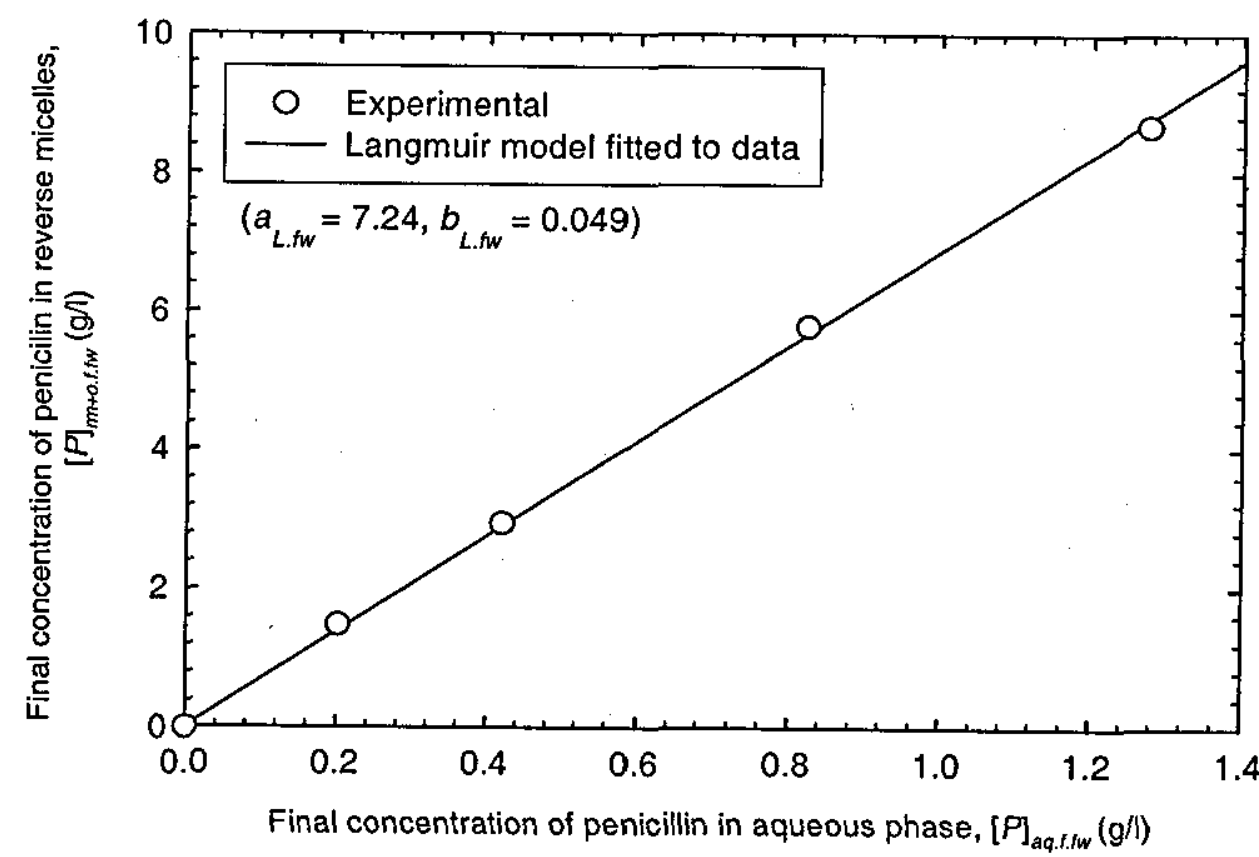

Figure A4.2-4: Langmuir model fitted to experimental data at $\mathrm{pH} 1.65$ and $[S]=267$ $\mathrm{g} / \mathrm{l}$. 


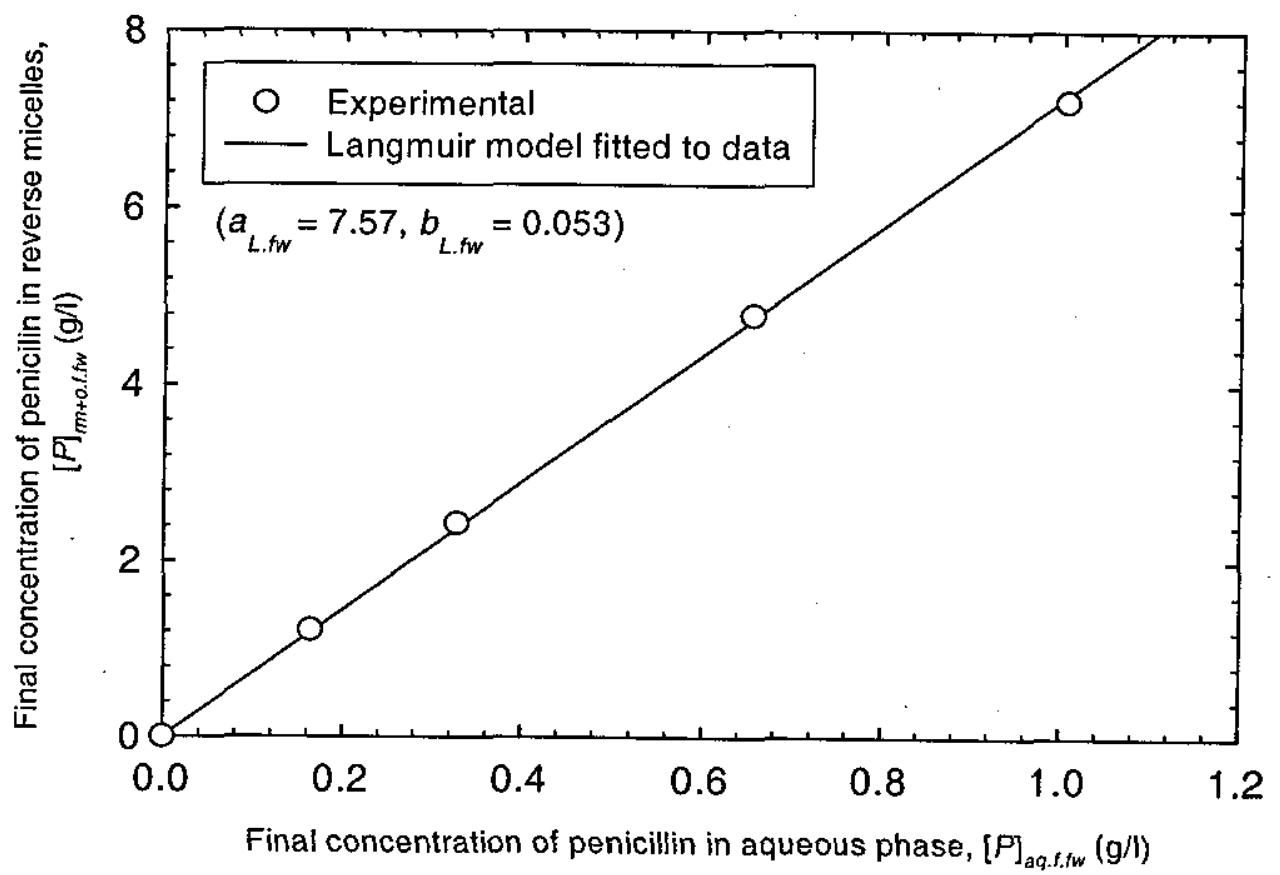

Figure A4.2-5: Langmuir model fitted to experimental data at $\mathrm{pH} 1.65$ and $[S]=445$ $\mathrm{g} / \mathrm{l}$.

\section{4-3 Brandani model fitted to experimental data}

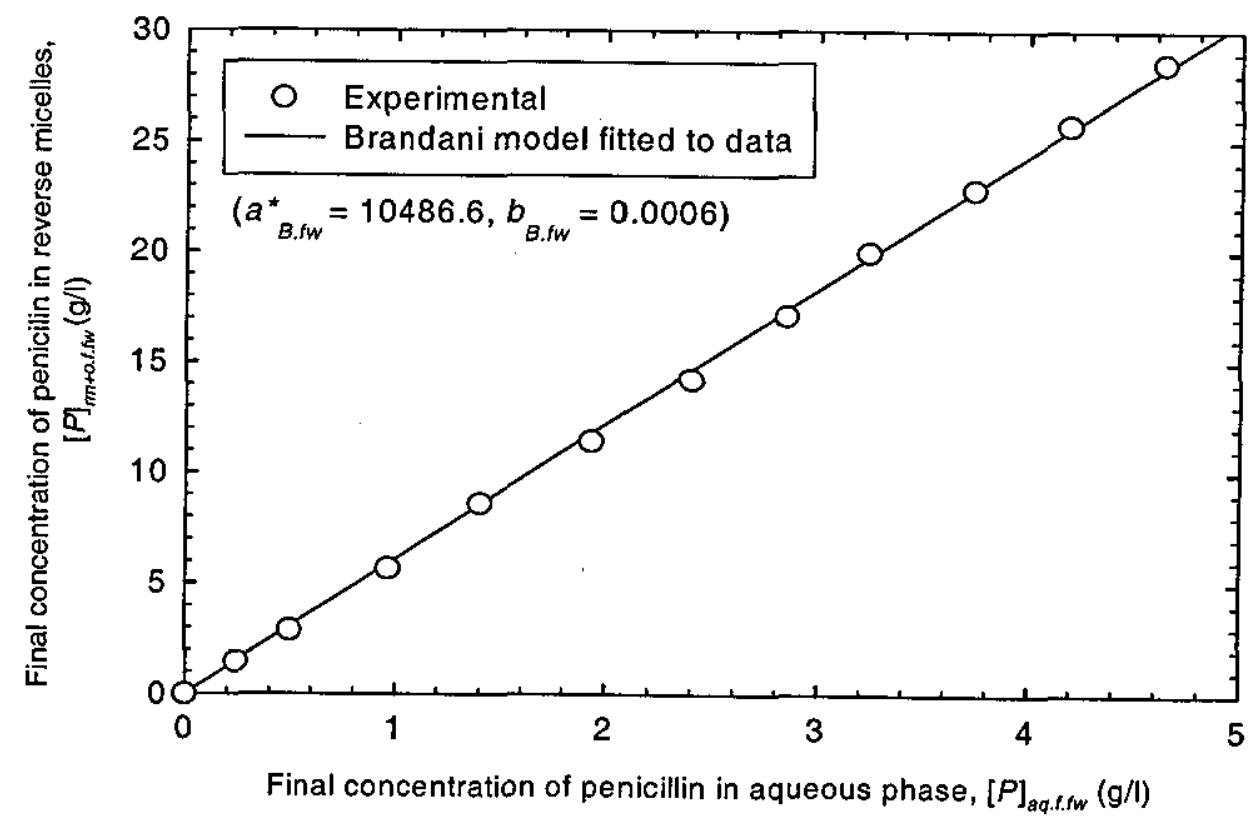

Figure A4.3-1: Brandani model fitted to experimental data at $\mathrm{pH} 7.6$ and $[S]=267$ g/l. $\left(a_{B . f w}^{*}=a_{B . f w}[S]\right)$. 


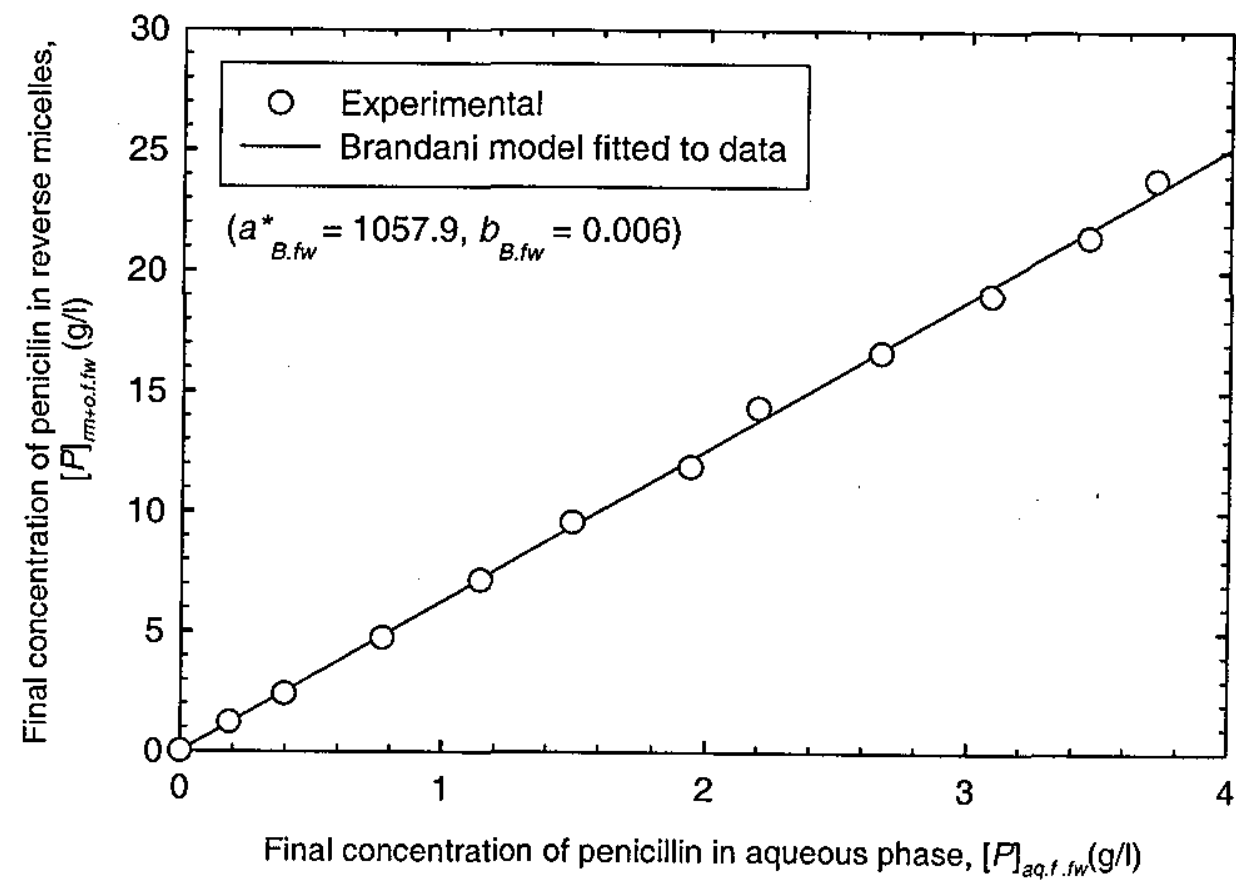

Figure A4.3-2: Brandani model fitted to experimental data at $\mathrm{pH} 7.6$ and $[S]=445$ g/l. $\left(a_{B . f w}^{*}=a_{B . f w}[S]\right)$.

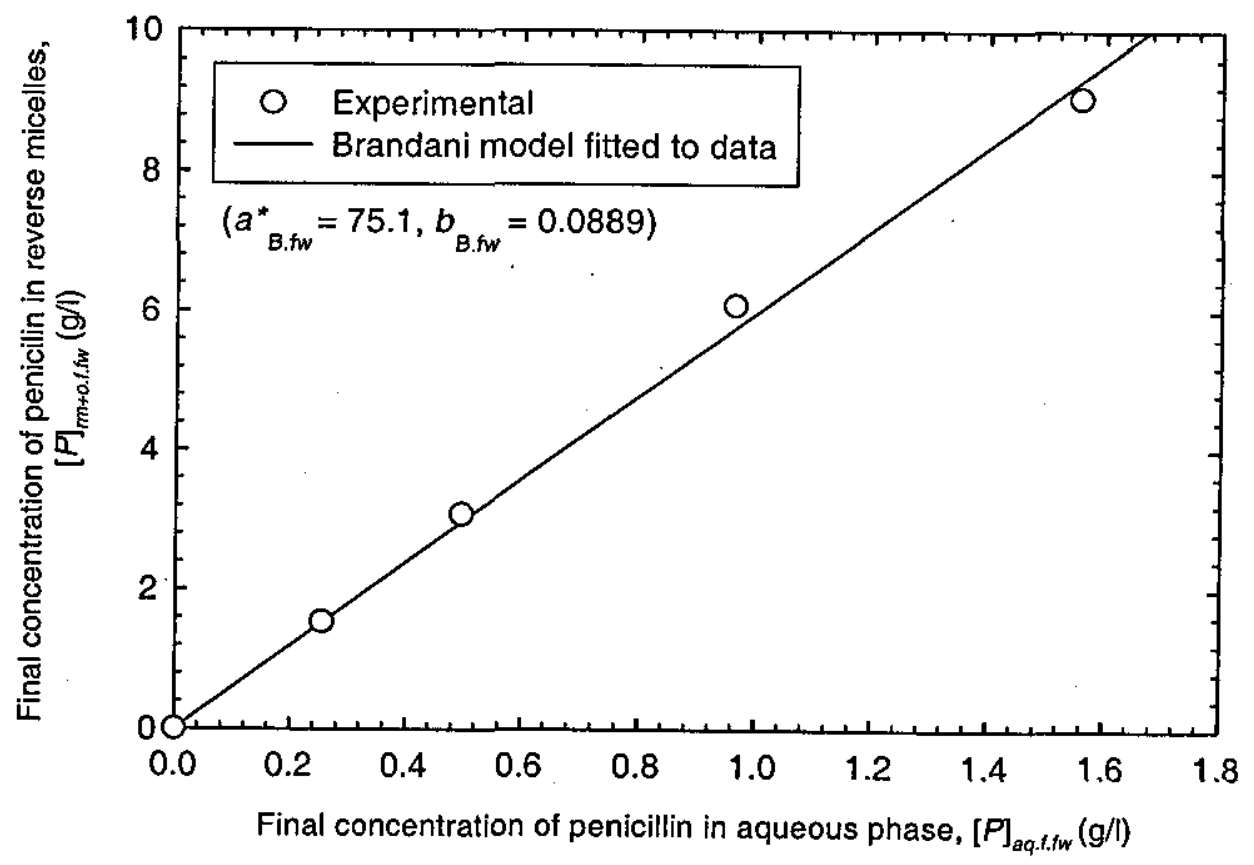

Figure A4.3-3: Brandani model fitted to experimental data at $\mathrm{pH} 1.65$ and $[S]=88$ g/l. $\left(a_{B . f w}^{*}=a_{B . f w}[S]\right)$. 


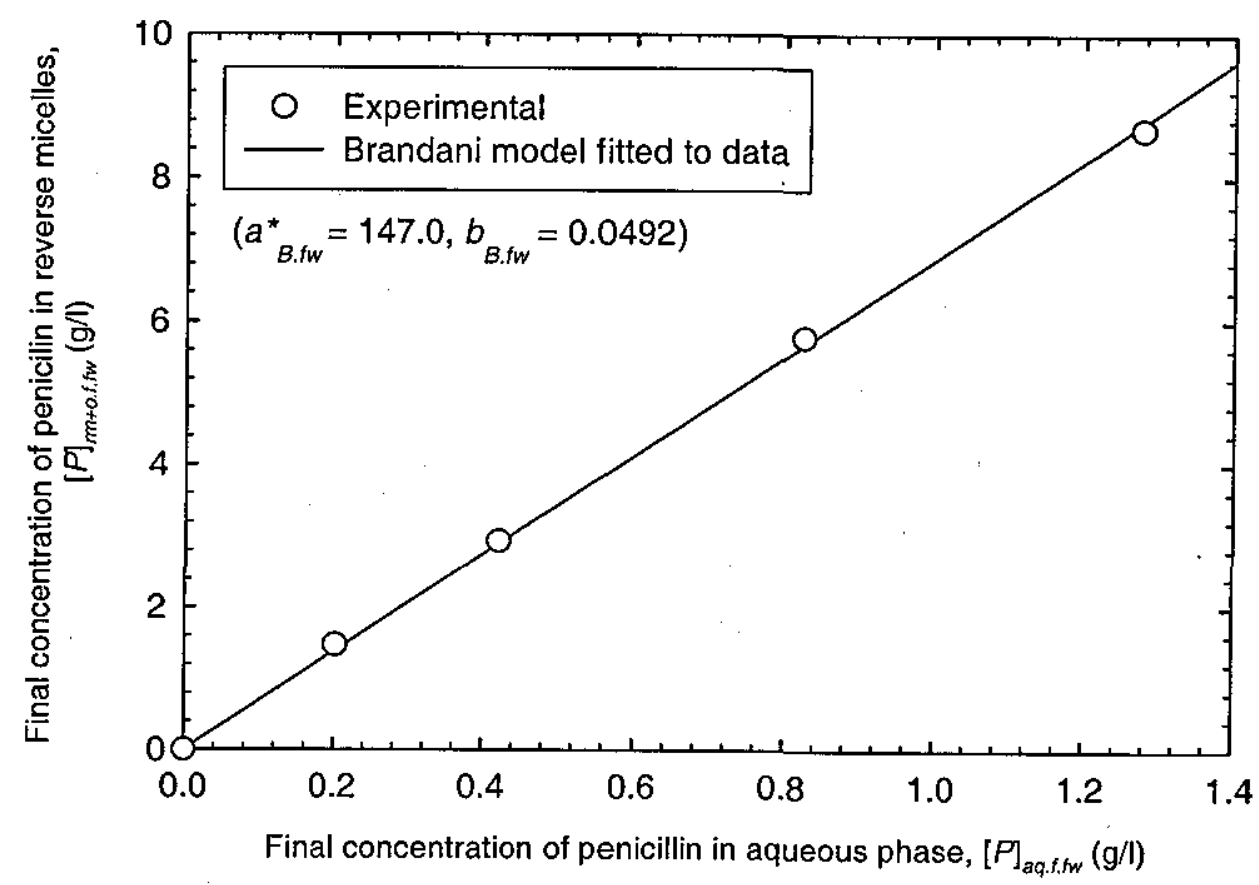

Figure A4.3-4: Brandani model fitted to experimental data at $\mathrm{pH} 7.6$ and $[S]=267$ g/l. $\left(a_{B . f w}^{*}=a_{B . f w}[S]\right)$.

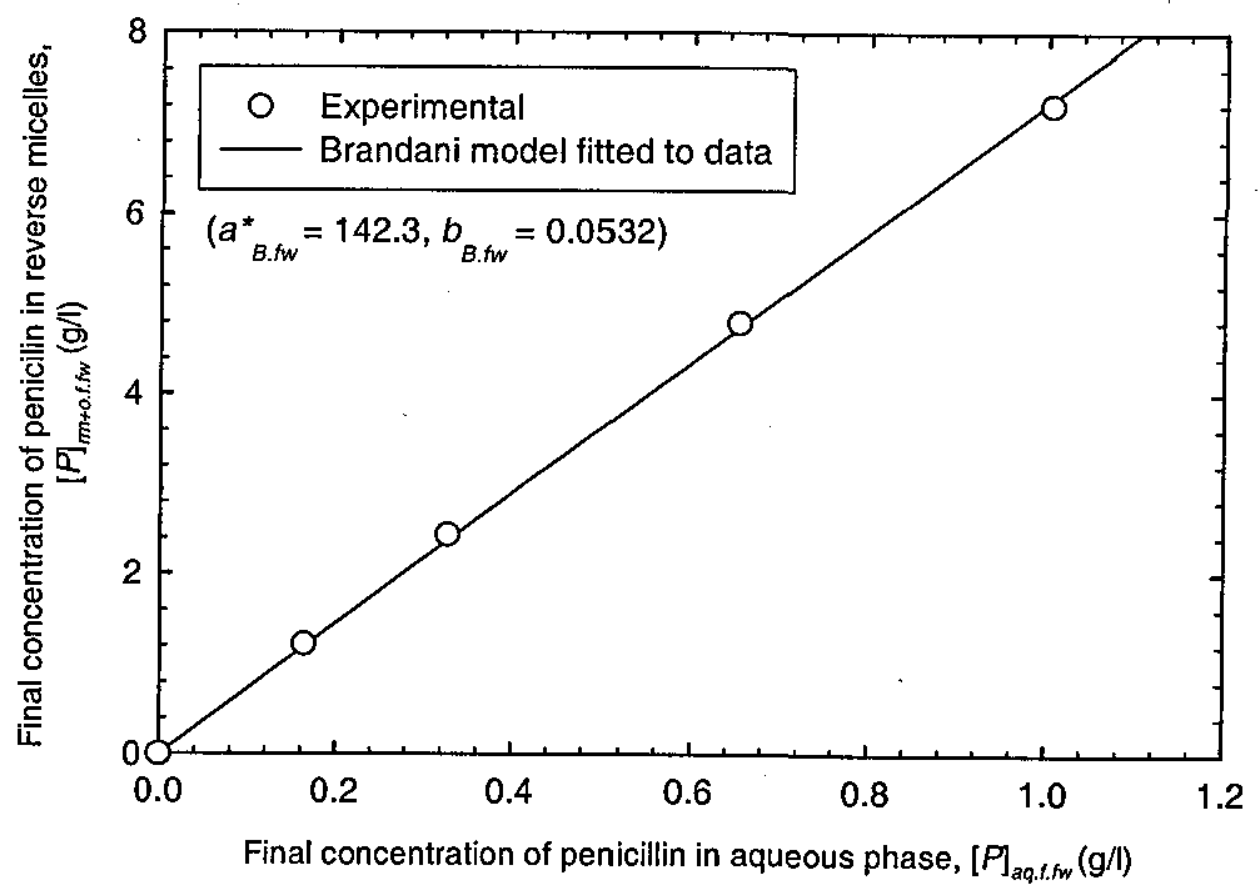

Figure A4.3-5: Brandani model fitted to experimental data at $\mathrm{pH} 1.65$ and $[S]=445$ g/l. $\left(a_{B . f w}^{*}=a_{B . f w}[S]\right)$. 


\section{Appendix 5}

Thermodynamic Framework for Backward Extraction 


\section{5-1 Isotherm of penicillin G for backward extraction}

a) $\mathrm{pH} 7.6$

Table A5-1: Isotherms of penicillin $\mathrm{G}$ at $\mathrm{pH} 7.6$ for backward extraction.

\begin{tabular}{|c|c|c|c|c|c|}
\hline \multicolumn{2}{|c|}{$[S]=88(\mathrm{~g} / \mathrm{l})$} & \multicolumn{2}{c|}{$[S]=267(\mathrm{~g} / \mathrm{l})$} & \multicolumn{2}{|c|}{$[S]=445(\mathrm{~g} / \mathrm{l})$} \\
\hline $\begin{array}{c}{[P]_{r m+o f . b w}} \\
(\mathrm{~g} / 1)\end{array}$ & $\begin{array}{c}{[P]_{a q, f . b w}} \\
(\mathrm{~g} / 1)\end{array}$ & $\begin{array}{c}{[P]_{r m+o f . b w}} \\
(\mathrm{~g} / \mathrm{l})\end{array}$ & $\begin{array}{c}{[P]_{\text {aq.f.bw }}} \\
(\mathrm{g} / \mathrm{l})\end{array}$ & $\begin{array}{c}{[P]_{r m+o f . b w}} \\
(\mathrm{~g} / \mathrm{l})\end{array}$ & $\begin{array}{c}{[P]_{\text {aq.f.bw }}} \\
(\mathrm{g} / \mathrm{l})\end{array}$ \\
\hline 0.00 & 0.00 & 0.00 & 0.00 & 0.00 & 0.00 \\
1.63 & 1.32 & 1.42 & 1.68 & 1.88 & 1.59 \\
4.39 & 1.68 & 4.37 & 2.28 & 5.47 & 2.31 \\
7.33 & 1.92 & 7.96 & 2.56 & 10.20 & 2.57 \\
10.19 & 2.25 & 11.87 & 2.56 & 15.05 & 2.77 \\
13.11 & 2.47 & 14.93 & 3.08 & 20.00 & 2.82 \\
16.18 & 2.74 & 18.40 & 3.43 & 24.83 & 3.08 \\
19.37 & 2.73 & 22.30 & 3.46 & 29.65 & 3.18 \\
22.67 & 2.73 & 26.26 & 3.44 & 34.88 & 3.18 \\
25.90 & 2.73 & 30.25 & 3.44 & 40.15 & 3.18 \\
29.06 & 2.74 & 34.12 & 3.44 & 45.38 & 3.18 \\
\hline
\end{tabular}

b) $\mathrm{pH} 1.65$

Table A5-2: Isotherms of penicillin $\mathrm{G}$ at $\mathrm{pH} 1.65$ for backward extraction.

\begin{tabular}{|c|c|c|c|c|c|}
\hline \multicolumn{2}{|c|}{$[S]=88(\mathrm{~g} / \mathrm{l})$} & \multicolumn{2}{c|}{$[S]=267(\mathrm{~g} / \mathrm{l})$} & \multicolumn{2}{|c|}{$[S]=445(\mathrm{~g} / \mathrm{l})$} \\
\hline $\begin{array}{c}{[P]_{\text {rm+o.f.bw }}} \\
(\mathrm{g} / \mathrm{l})\end{array}$ & $\begin{array}{c}{[P]_{\text {aq. } f . b w}} \\
(\mathrm{~g} / \mathrm{l})\end{array}$ & $\begin{array}{c}{[P]_{\text {rm+of.bw }}} \\
(\mathrm{g} / \mathrm{l})\end{array}$ & $\begin{array}{c}{[P]_{r m+o f . b w}} \\
(\mathrm{~g} / \mathrm{l})\end{array}$ & $\begin{array}{c}{[P]_{\text {aq.f.bw }}} \\
(\mathrm{g} / \mathrm{l})\end{array}$ & $\begin{array}{c}{[P]_{r m+o f . b w}} \\
(\mathrm{~g} / \mathrm{l})\end{array}$ \\
\hline 0.00 & 0.00 & 0.00 & 0.00 & 0.00 & 0.00 \\
1.85 & 1.30 & 1.51 & 1.74 & 2.28 & 1.45 \\
4.67 & 1.69 & 4.55 & 2.34 & 5.80 & 2.24 \\
7.83 & 1.75 & 8.19 & 2.58 & 10.44 & 2.55 \\
\hline
\end{tabular}




\section{5-2 Langmuir model fitted to experimental data}

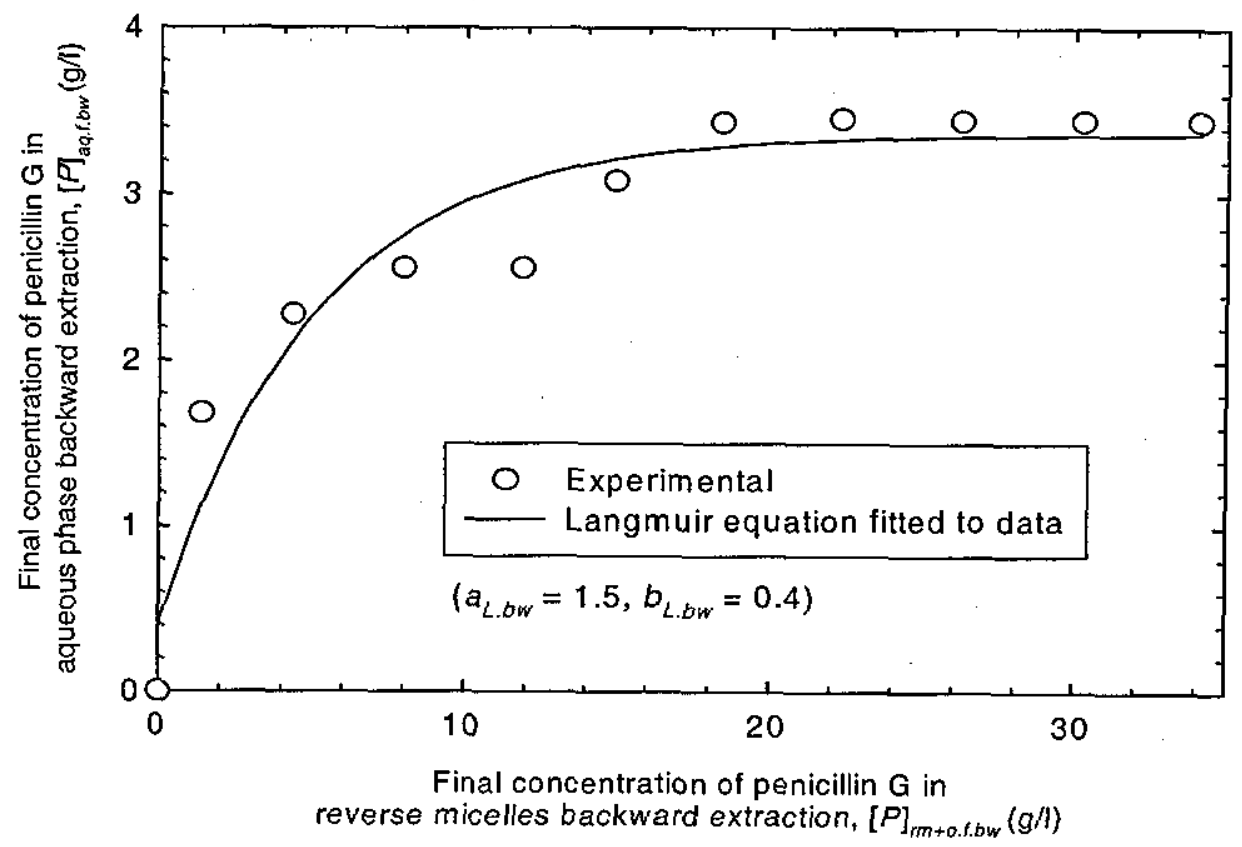

Figure A5.2-1: Langmuir model fitted to experimental data at $\mathrm{pH} 7.6$ and $[S]=267 \mathrm{~g} / 1$ for backward extraction.

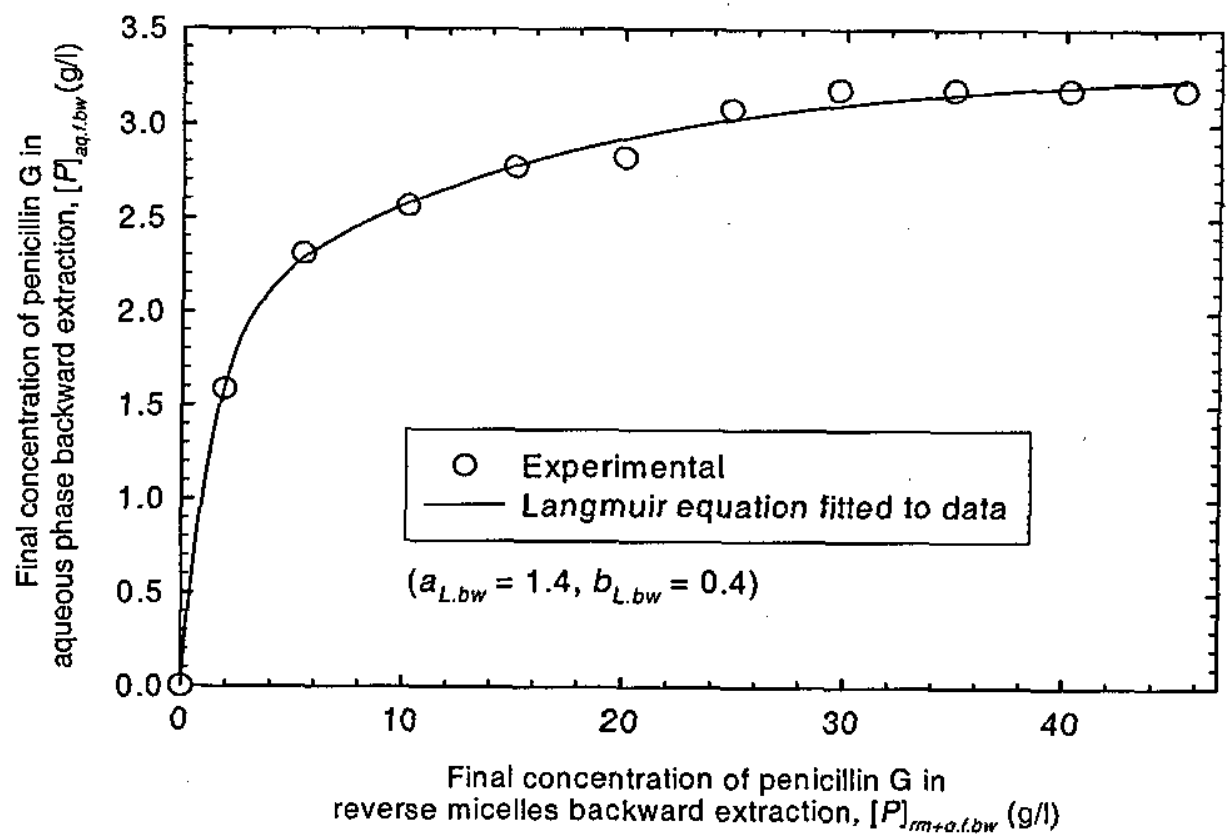

Figure A5.2-2: Langmuir model fitted to experimental data at $\mathrm{pH} 7.6$ and $[S]=445 \mathrm{~g} / 1$ for backward extraction. 


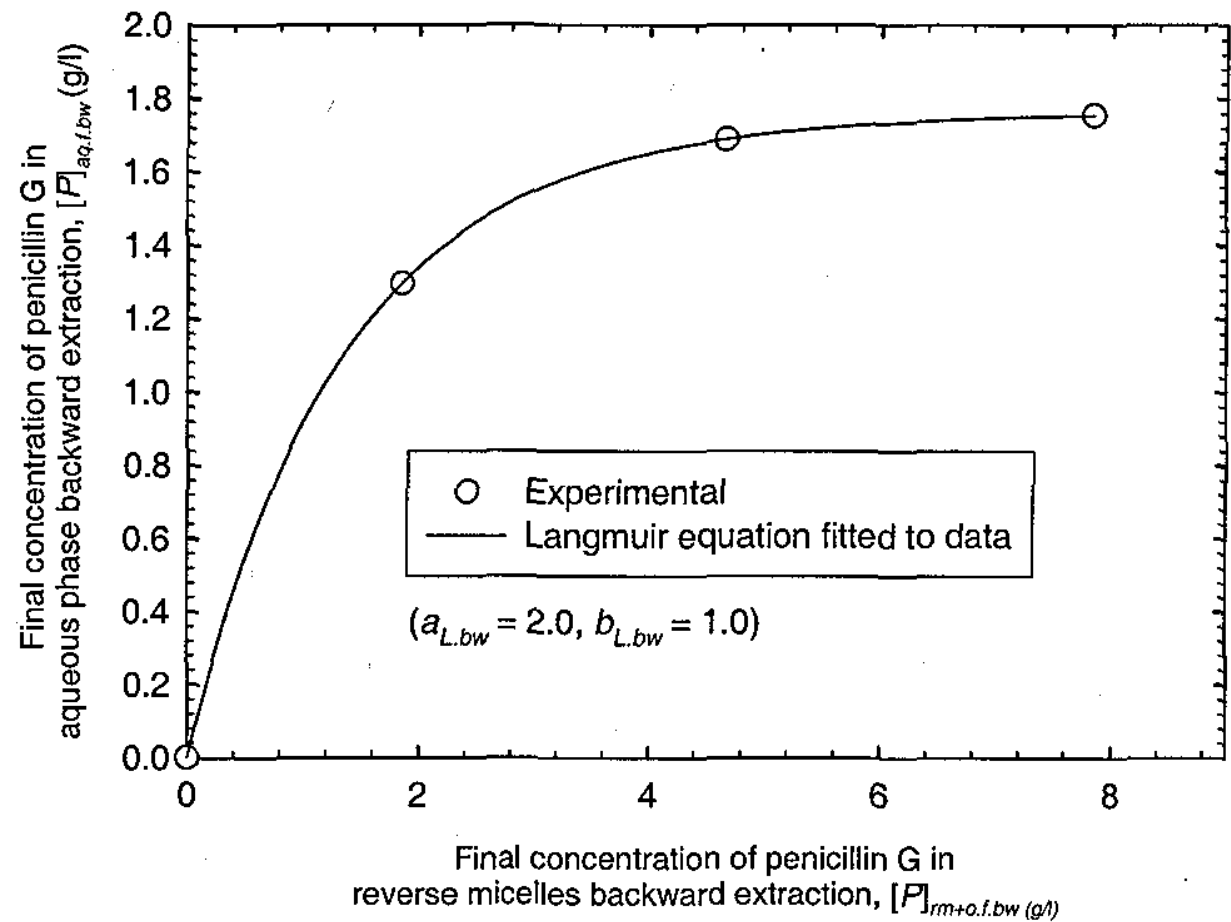

Figure A5.2-3: Langmuir model fitted to experimental data at $\mathrm{pH} 1.65$ and $[S]=88 \mathrm{~g} / 1$ for backward extraction.

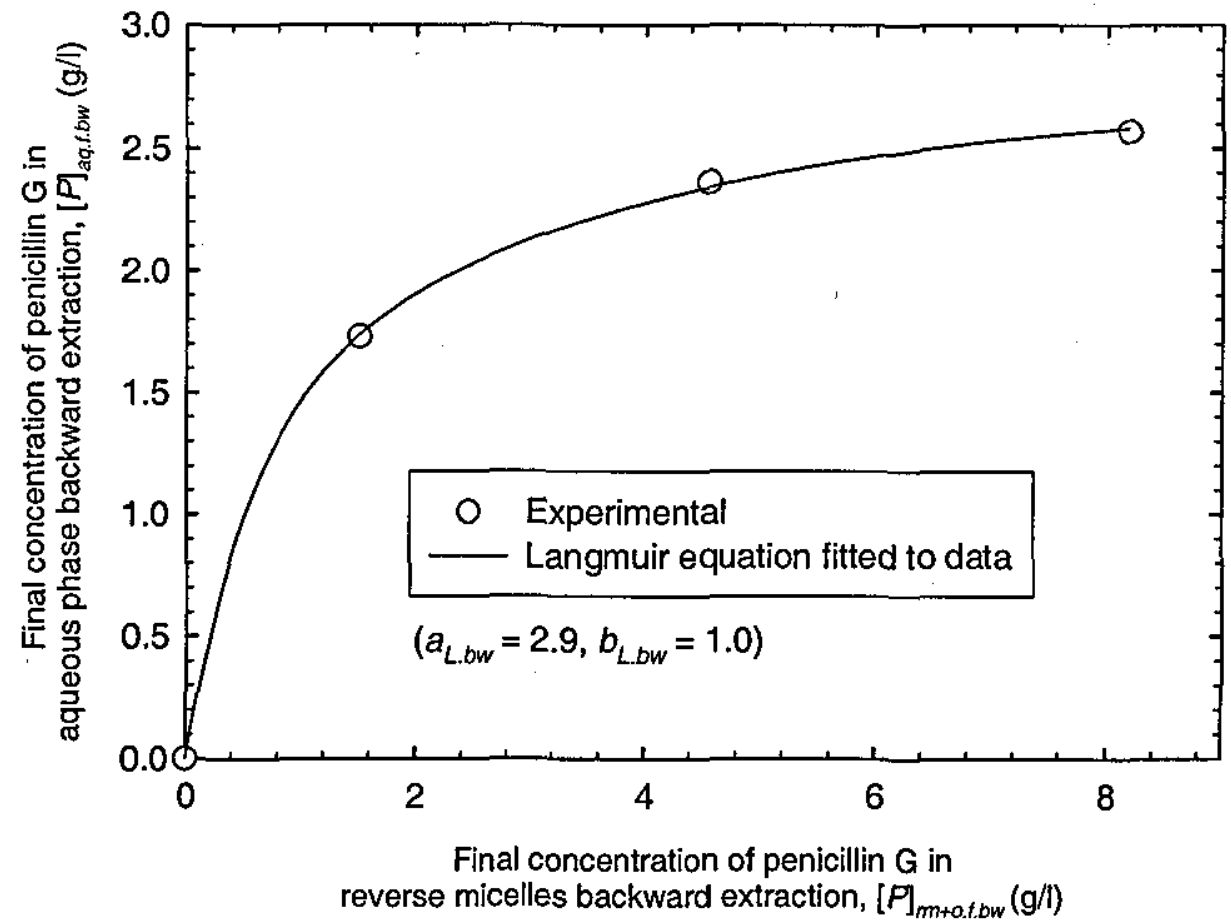

Figure A5.2-4: Langmuir model fitted to experimental data at $\mathrm{pH} 1.65$ and $[S]=267 \mathrm{~g} / \mathrm{l}$ for backward extraction. 


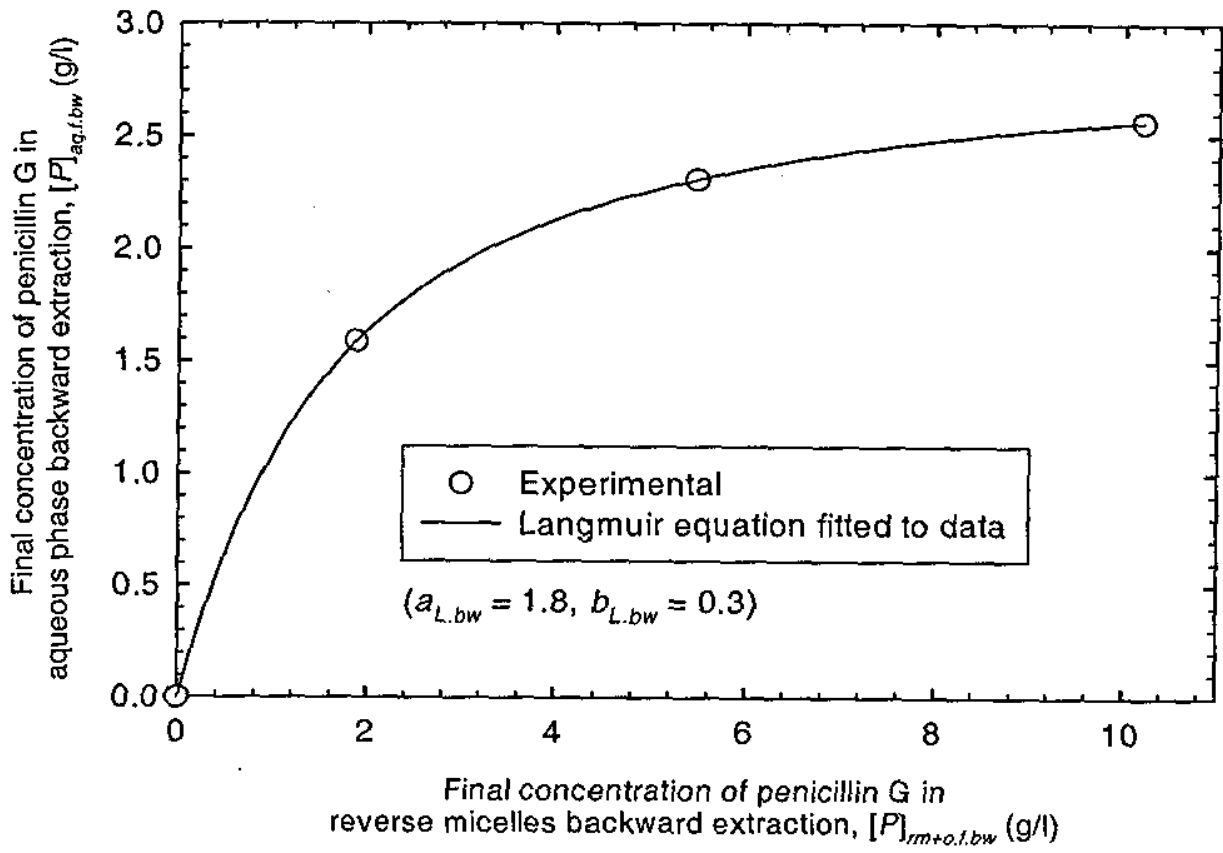

Figure A5.2-5: Langmuir model fitted to experimental data at $\mathrm{pH} 1.65$ and $[S]=445 \mathrm{~g} / 1$ for backward extraction.

\section{5-3 Brandani model fitted to experimental data}

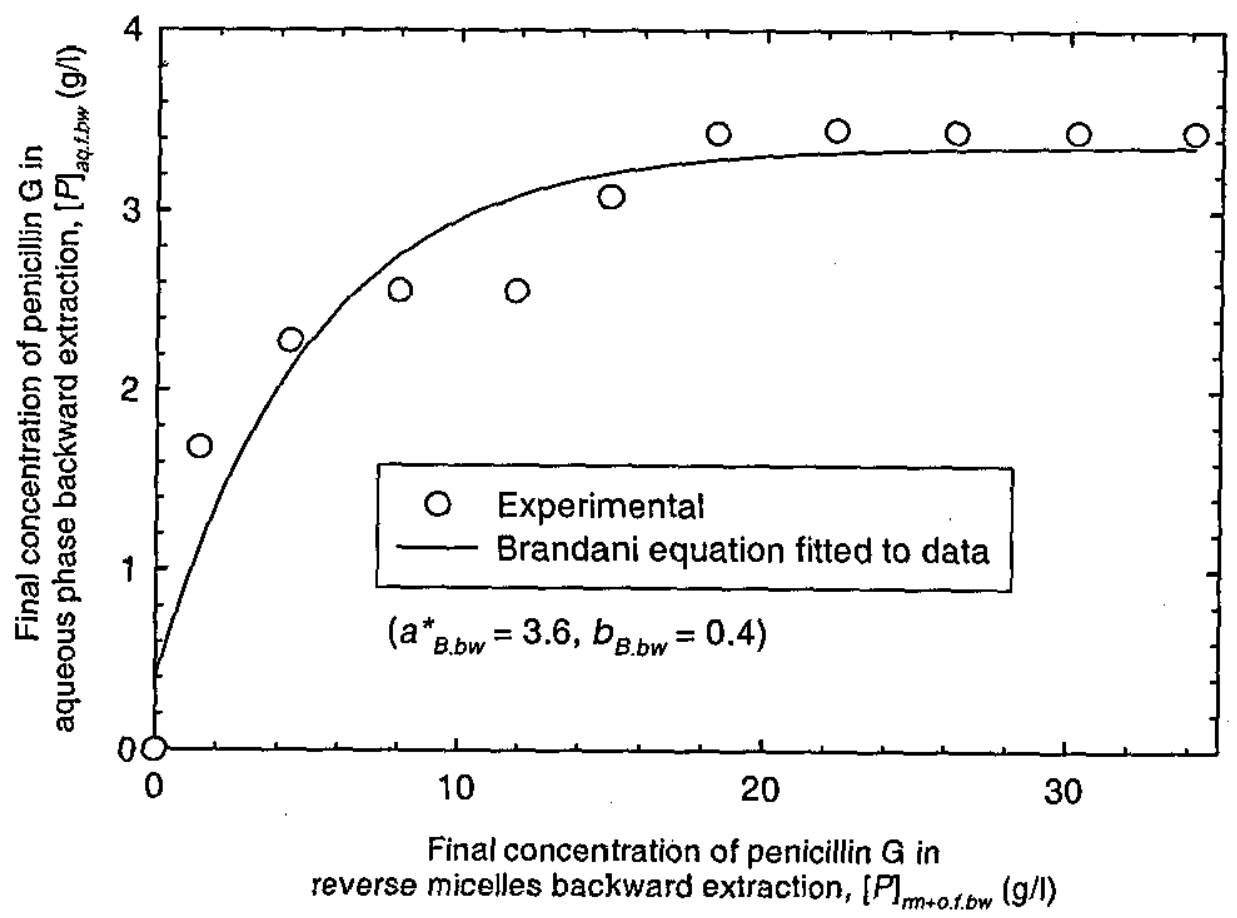

Figure A5.3-1: Brandani model fitted to experimental data at $\mathrm{pH} 7.6$ and $[S]=267 \mathrm{~g} / 1$ for backward extraction. ( $\left.a^{*}{ }_{\text {B.bw }}=a_{B . b w}[S]\right)$. 


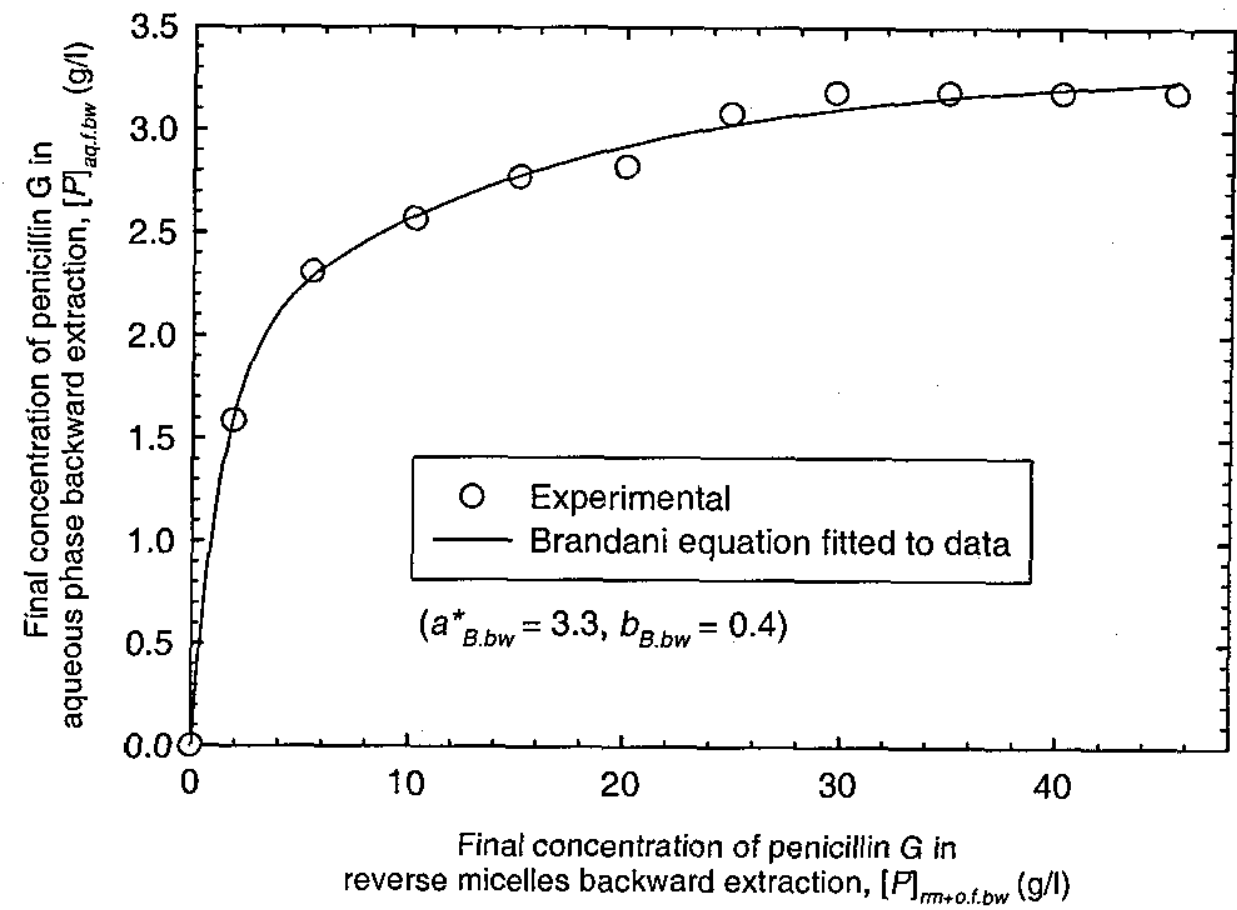

Figure A5.3-2: Brandani model fitted to experimental data at $\mathrm{pH} 7.6$ and $[S]=445 \mathrm{~g} / \mathrm{l}$ for backward extraction. $\left(a_{B \cdot b w}^{*}=a_{B . b w}[S]\right)$.

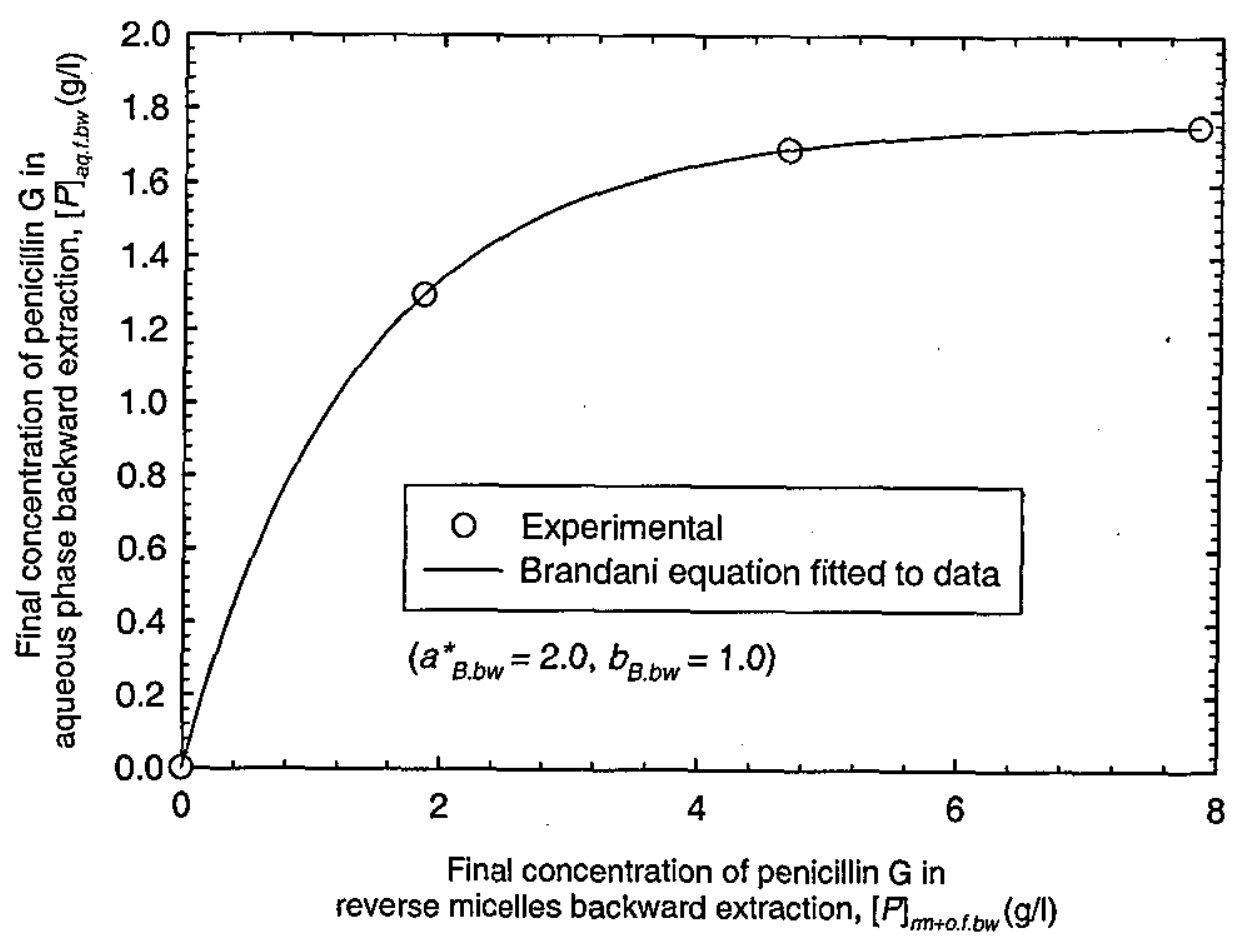

Figure A5.3-3: Brandani model fitted to experimental data at $\mathrm{pH} 1.65$ and $[S]=88 \mathrm{~g} / \mathrm{l}$ for backward extraction. $\left(a_{B \cdot b w}^{*}=a_{B, b w}[S]\right)$. 


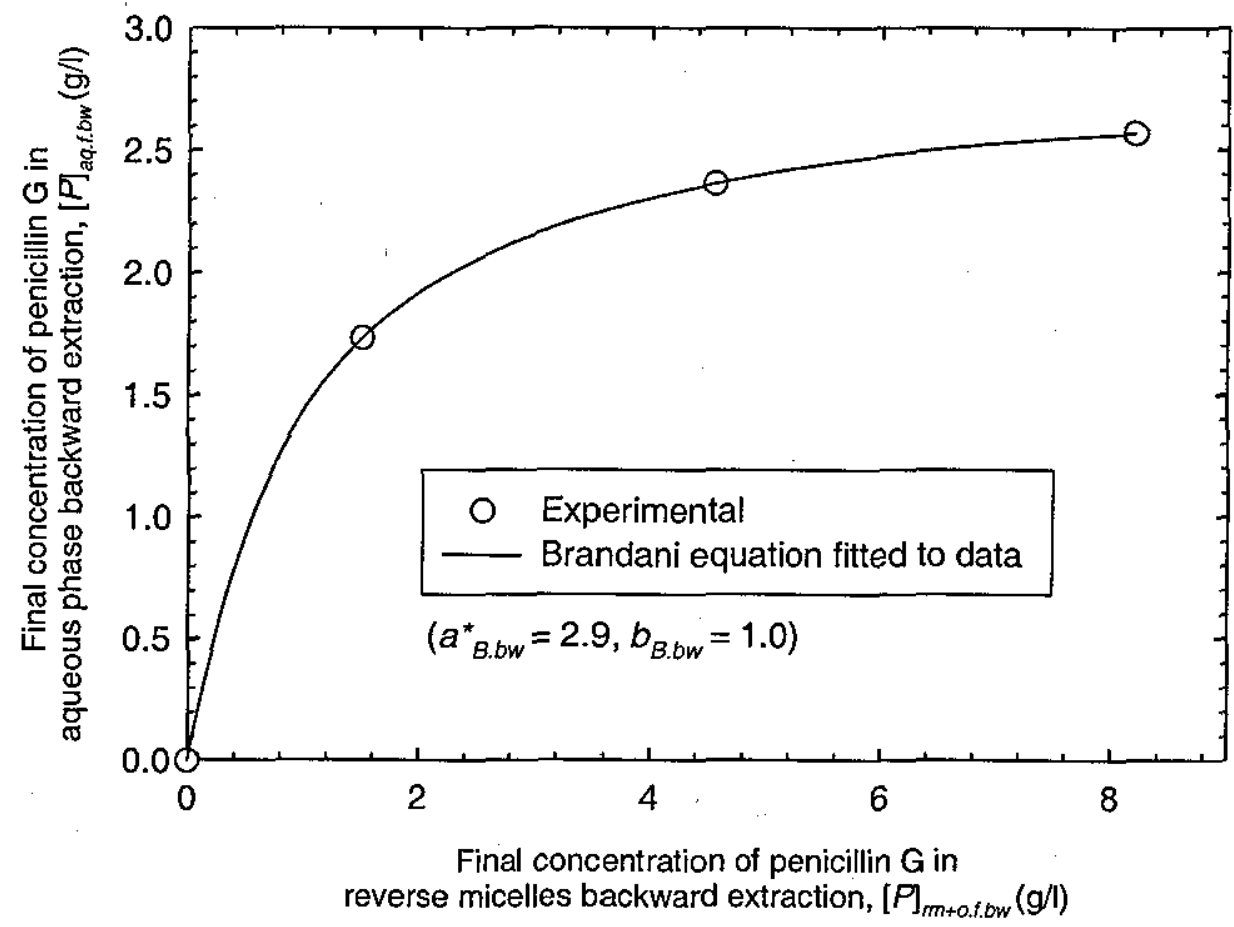

Figure A5.3-4: Brandani model fitted to experimental data at $\mathrm{pH} 1.65$ and $[S]=267 \mathrm{~g} / 1$ for backward extraction. $\left(a^{*}{ }_{B \cdot b w}=a_{B \cdot b w}[S]\right)$.

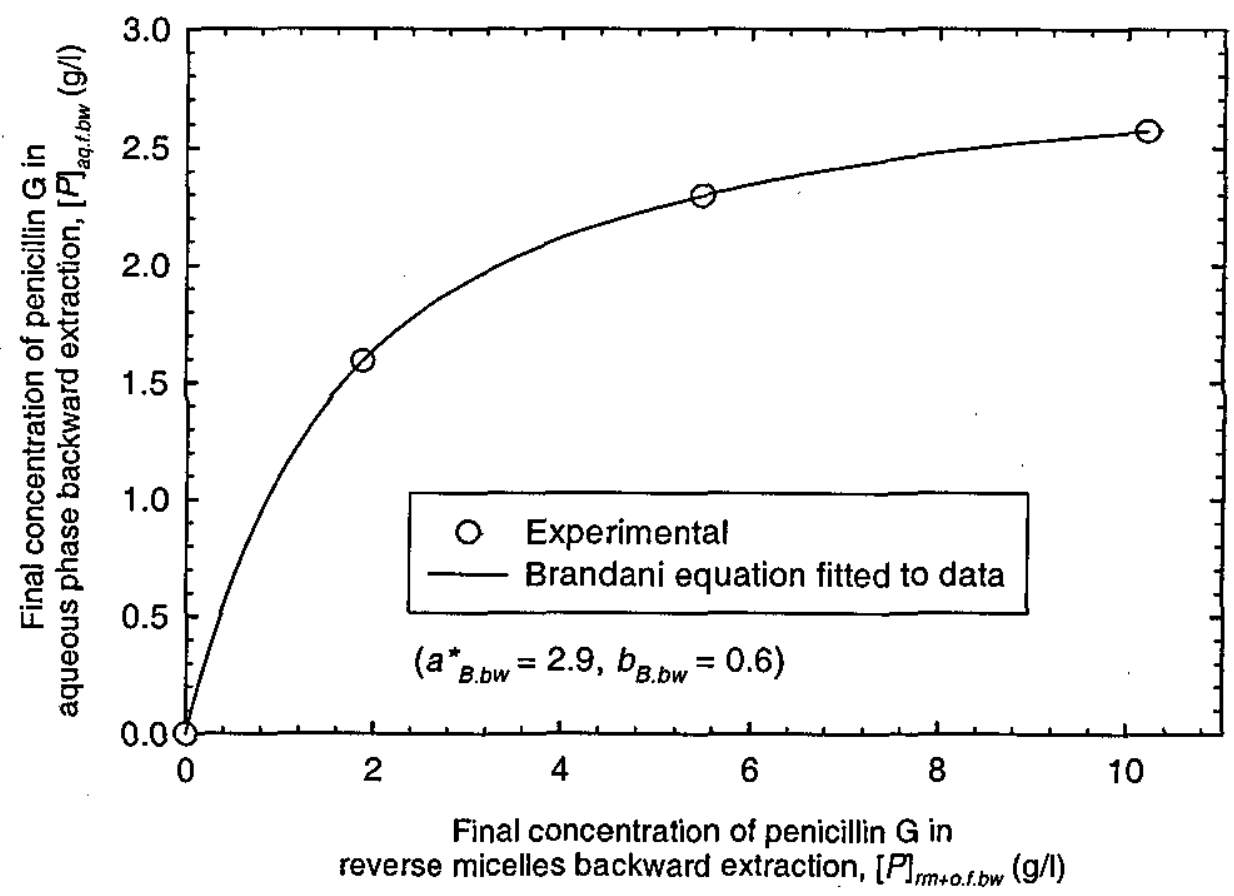

Figure A5.3-5: Brandani model fitted to experimental data at $\mathrm{pH} 1.65$ and $[S]=445 \mathrm{~g} / \mathrm{l}$ for backward extraction. $\left(a_{B \cdot b w}^{*}=a_{B, b w}[S]\right)$. 
Appendix 6

List of Publications Arising from the Research 
Refereed Journals

1. Mohd-Setapar S.H., Wakeman R.J and Tarleton E.S., Penicillin G solubilisation into AOT reverse micelles. Chemical Engineering Research Design, submitted.

2. Mohd-Setapar S.H., Wakeman R.J. and Tarleton E.S., Backward extraction of penicillin G using reverse micelles. Chemical Engineering Research Design, in preparation.

\section{Conference Proceedings}

3. Mohd-Setapar S.H., Wakeman R.J. and Tarleton E.S., 2008, Separation of pharmaceutical products with reverse micelles, $10^{\text {th }}$ World Filtration Congress (WFC 10), Leipzig, Germany, 14-18 ${ }^{\text {th }}$ April.

4. Mohd-Setapar S.H., Wakeman R.J. and Tarleton E.S., Reverse micellar liquidliquid extraction of a pharmaceutical product, Icheme Symposium - What's New in Fluid Separations?, GlaxoSmithKline, Stevanage, UK, $30^{\text {th }}$ May.

5. Mohd-Setapar S.H., Wakeman R.J and Tarleton E.S., 2008, Penicillin G separation by microemulsion, Icheme Forum - Chemical |Engineering 2008 ChemEng08, NEC, Birmingham, UK, 28-30 October. 
6-1 Paper submitted to the joumal of Chemical Engineering Research Design

\title{
PENICILLIN G SOLUBILISATION INTO AOT REVERSE MICELLES
}

\author{
S.H. Mohd-Setapar ${ }^{*}$, R.J. Wakeman and E.S. Tarleton \\ Advanced Separation Processes Group, Department of Chemical Engineering, \\ Loughborough University, Loughborough, Leicestershire, LE11 3TU, UK.
}

\begin{abstract}
Extraction of penicillin $\mathrm{G}$ between from an aqueous phase into an organic phase containing AOT reverse micelles has been investigated. The extraction is influenced by the initial penicillin $\mathrm{G}$ concentration, the salt type and concentration in the aqueous phase, $\mathrm{pH}$, and surfactant concentration. The results show that penicillin is an interfacially active compound that interacts with AOT, with the interfacial association being dependent on both $\mathrm{pH}$ and surfactant concentration. When the concentration ratio $[P]_{a q} /[S]$ is high precipitation of the penicillin occurs. The distribution coefficient favours transfer of the penicillin into the reverse micelles at moderate AOT concentrations. The distribution coefficient at infinite dilution, $K^{\infty}$, is shown to be a function of both $\mathrm{pH}$ and surfactant concentration; similar trends in the value of $K^{\infty}$ were observed at different $\mathrm{pH}$ values; $K^{\infty}$ decreases as the surfactant concentration is increased. Reverse micelle formation affects the volume of water transferred into the organic phase; from measurements of the water transferred, the size of the reverse micelles was estimated to be about $3 \mathrm{~nm}$ and the number of AOT molecules per reverse micelle about 360 .
\end{abstract}

Keywords: anionic surfactant, extraction, partition coefficient, penicillin, reverse micelle', solubilisation, water content.

*Coresponding aouthor email address S.H.Mohd-Setapar@lboro.co.uk 


\section{INTRODUCTION}

Penicillin G, also known as benzyl penicillin, is a widely used antibiotic and it has also been used as a raw material for semisynthetic penicillin [1]. Various separation methods such as solvent extraction, ion-exchange, chromatography, crystallisation, or a combination of these, have been used for the recovery of antibiotics, however solvent extraction due to its effectiveness and favourable economics is the most widely used [2]. Solvent extraction has several difficulties which can cause problems such as final product contamination, low extraction yield, clogging of equipment, and high solvent losses $[1,3,4]$. Therefore new separation techniques have been studied to overcome the difficulties experienced whilst using conventional solvent extraction for penicillin G. Some of the new extraction techniques that have been subjects of experimental studies include ultrafiltration and nanofiltration $[3,5]$, liquid surfactant membranes [6], and reactive extraction [1].

Separation processes using reverse micelles are based on conventional liquid-liquid extraction techniques. They are easy to scale up, have a high efficiency, can be made selective, have a low energy demand, and can be operated continuously [7,8,10-14]. They can also offer moderate thermal conditions for processing of biomaterials $[5,9]$. The method is a kind of solvent extraction using reverse micelles as extractant [10]. Reverse micelles, also known as water-in-oil microemulsions, consist of three components: amphiphilic surfactant molecules, an aqueous phase, and a non-polar organic solvent. The polar heads of the surfactant molecules are directed towards the interior of a water-containing sphere, whereas the aliphatic tails are oriented toward the non-polar organic phase. Many authors have investigated the partitioning of proteins between two immiscible liquids, and the considerable success of protein partitioning has provided the motivation for using the technique for antibiotics; in our case penicillin G. Reverse micelles have the ability to extract a variety of biomolecules such as proteins[10,11,15-19] and enzymes[16,19] into nanometre-size water pools surrounded by a monolayer of surfactant.

To date most research on the purification of antibiotic molecules has focussed on those dissolved in an aqueous solvent. For example, Nabais and Cardoso [3] studied the purification of penicillin $\mathrm{G}$ from fermentation broth by ultrafiltration, and $\mathrm{Hu}$ and Gulari [12] studied the extraction of aminoglycoside antibiotics such as neomycin and gentamycin using reverse micelles. These authors did not give consideration to modelling of partitioning of the antibiotic between the phases, although thermodynamic studies are important for designing downstream processing [20]. Although thermodynamic models have been applied to protein solubilisation into reverse micelles [20-26], there does not seem to have been any thermodynamic studies of penicillin $\mathrm{G}$ solubilisation. The apparent success of research on protein extraction from the aqueous phase using reverse micelles provides motivation to study the solubilisation of penicillin $\mathrm{G}$, and in this work we investigate the parameters that affect solubilisation of penicillin $\mathrm{G}$ molecules into reverse micelles that lie in the organic phase.

\section{MATERIALS AND METHODS}


Reagent grade sodium di-2-ethylhexyl sulfosuccinate (AOT) was used as the surfactant, the organic solvent used for the reversed micelles was analytical grade isooctane, and the biomolecule was penicillin $\mathrm{G}$ sodium salt. $\mathrm{CaCl}_{2}$ and $\mathrm{KCl}$ were used as salts in the aqueous phase. All chemicals were supplied from Sigma Aldrich UK and were used as received. All experiments were conducted at a temperature of $23 \pm 1^{\circ} \mathrm{C}$.

$5 \mathrm{ml}$ of aqueous solution containing penicillin $\mathrm{G}$ and $\mathrm{KCl}$ was contacted with an equal volume of isooctane containing AOT; the phase transfer experiments are shown diagrammatically in Figure 1. In a sequence of experiments different AOT concentrations, $\mathrm{pH}$, and penicillin $\mathrm{G}$ concentrations were used. The $\mathrm{pH}$ of the aqueous phase was adjusted using either $\mathrm{NaOH}$ or $\mathrm{KOH}$. The solutions were then mixed for 10 minutes using a magnetic stirrer at $400 \mathrm{rpm}$. Higher stirrer speeds and longer stirring periods were not used since a stable emulsion formed under those conditions. The mixture then was left for the phases to separate. After 24 hours of settling (some samples needed more than 24 hours to produce clear interface between the aqueous and organic phases), samples of the organic phase were removed carefully using a pipette or a syringe. For each experiment a blank solution was prepared by contacting an aqueous solution without penicillin $\mathrm{G}$ with an equal volume of initial micellar solution. After settling, all samples of both phases were transparent to the naked eye. All experiments were replicated from two to four times in order to assess the reproducibility of the results.

The concentration of penicillin $\mathrm{G}$ in the separated aqueous phases was measured using the Kjeldahl method. The concentration of penicillin $\mathrm{G}$ in the organic phase was determined using mass balance. A Buchi Nitrogen Determination System. (consisting of a distillation unit, a scrubber unit, and a digestion unit) was used to measure the nitrogen content of the samples; since nitrogen existed in only the penicillin $\mathrm{G}$, the nitrogen contents were calibrated against the penicillin $\mathrm{G}$ concentration. The water content in the reverse micellar phase was measured using a Karl Fisher (KF) titrator (Mitsubishi Kasei Corp.). The readings from the KF titrator, given as percent weight of water in the sample, were then converted to the water uptake, $W_{o}$, which is the molar ratio of water to surfactant in the organic phase, $\left[\mathrm{H}_{2} \mathrm{O}\right] /[\mathrm{AOT}]$. Surface tension measurements were performed using a Wilhelmy Ring connected to a tensiometer (White Electrical Instrument Co. Ltd).

\section{THERMODYNAMIC FRAMEWORK FOR SOLUBILISATION}

Brandani et al. [25] developed an adsorption model that consisted of an aqueous phase in equilibrium with an organic phase with many identical sites made up of reverse micelles onto each of which one molecule of the adsorbing material (solute) can be entrapped. The concentration of solute in the reverse micelle was then be expressed as:

$$
[P]_{r m+o . f}=\frac{a b[S][P]_{a q . f}}{1+b[P]_{a q . f}}
$$

where $a$ and $b$ are adjustable parameters; $b$ is a parameter characteristic of the inner surface of reversed micelles, the solute, and the temperature, but it is independent of 
$[P]_{\text {aq. } f .}[S]$ is the concentration of the surfactant. Using this model, the distribution coefficient depends on both $[S]$ and $[P]_{a q . f}$ and can be written as:

$$
K=\frac{[P]_{m+o . f}}{[P]_{a q . f}}=\frac{a b[S]}{1+b[P]_{a q . f}}
$$

At infinite dilution $[P]_{a q . f} \rightarrow 0$ and

$$
K^{\infty}=a b[S]
$$

or

$$
\ln K^{\infty}=\ln a+\ln b+\ln [S]
$$

A slightly different equation based on the mass-action principle was derived by Woll and Hatton [27] which indicated a $\mathrm{pH}$ dependency:

$$
\therefore \ln K^{\infty}=\ln a+\ln b+(c+d \cdot p H) \ln [S]
$$

This model assumed a pseudo-chemical equilibrium for complexation of protein molecules with the hydrophobic tails of reverse micelles as empty sites. Also using the mass-action principle, Rabie and Vera [22] developed an ion exchange model for protein partitioning, based on the assumption that at equilibrium the concentration of the protein in both the aqueous and reverse micellar phases are the same and that the activities of components in a solution are equal to their concentration.

Other models using vacancy solution theory based on surface pressure have been derived. On the premise that one vacancy solution represents the aqueous phase and the other the reverse micellar phase, Haghtalab and Osfouri [20] incorporated surface pressure to model protein adsorption in reverse micelles and the non-ideality of the system was expressed in terms of the Wilson activity coefficient model. The concentration of protein remaining in the aqueous phase was obtained as

$$
[P]_{a q . f}=\left[\frac{1}{b} \frac{\theta_{1}}{1-\theta_{1}}\right] f\left(\Lambda_{12}, \Lambda_{21}, \theta_{1}\right)
$$

where $\Lambda_{12}$ and $\Lambda_{21}$ are Wilson parameters and $\theta_{1}$ is the fractional coverage of the protein molecules. The term in square brackets is exactly the same as the Langmuir equation, and $f\left(\Lambda_{12}, \Lambda_{21}, \theta_{1}\right)$ is may be regarded as a correction factor to the Langmuir isotherm. Their data showed that the values of the Wilson parameters are $\mathrm{pH}$ dependent, which could reasonably be expected since the surface charge on the protein (BSA in their case) varied from negative at $\mathrm{pH}<\mathrm{pI}$ to positive at $\mathrm{pH}>\mathrm{pI}$.

\section{RESULTS AND DISCUSSION}

At equilibrium, the penicillin is distributed between four solubilisation sites: (i) the aqueous phase continuum, (ii) the water pools lying within the reverse micelles in the 
organic continuum, (iii) the organic continuum, and (iv) at the reversed micellar interface between the organic phase and the water pool (see Figure 2).

The critical micelle concentration (CMC), the lowest concentration of surfactant needed for reversed micelle formation, of the AOT in isooctane was measured using a tensiometer and the results are shown in Figure 3. For the experiments without penicillin, the AOT was mixed into a sample of isooctane and the surface tension of the mixture measured. For those experiments where penicillin was present, the penicillin was extracted from an aqueous solution and the organic and aqueous solutions separated before the surface tension of the organic phase was measured. The concentration of penicillin is therefore different for each data point, and corresponds to its equilibrium partitioning between the phases.

Use of a stirrer speed greater than $400 \mathrm{rpm}$ caused formation of an emulsion that made clear phases separation difficult. In some cases the emulsion was short lived and disappeared after 24 hours settling time. The effect of the settling period on the concentration of penicillin $\mathrm{G}$ in the organic phase at different AOT concentrations was studied. From our observations there were no significant differences between 24 hours and one week settling times, indicating that the penicillin solubilisation had reached an equilibrium state; more than 24 hours settling time did not have an effect on the extraction efficiency.

\section{Interfacial Association and Phase Volume Changes}

When experiments were carried out using different AOT concentrations, there was an increase in the volume of the organic phase that was proportionate to a decrease of the aqueous phase volume, as shown in Figure 4. At AOT concentrations less than the $\mathrm{CMC}$, there was little or no visible increase in the volume of the organic phase. When the AOT concentration was increased to above about $40 \mathrm{~g} / \mathrm{l}$, the effect of reverse micelle formation showed itself by an increase in the volume of the organic phase as water was transferred into the reverse micellar structures.

Leodidis and Hatton [26] suggested, based on arguments stemming from Gibbs free energy of transfer of a solute from water to the surfactant interface, that an interfacial partition coefficient could be calculated on the basis of solute concentration measurements of the initial and final aqueous phases. For a solute that does not associate with the revere micellar interface $[P]_{a q . f}=[P]_{a q . i}$, and deviations from this line imply interfacial association of the solute. Figure 5 is a plot of the $[P]_{a q . f}$ versus $[P]_{\text {aq. } i}$ data for the AOT-penicillin system, and illustrates that penicillin $\mathrm{G}$ is an interfacially active molecule at all AOT concentrations. The slope of the data points indicates there is the least surface activity at concentration of an AOT concentration of $88 \mathrm{~g} / \mathrm{l}$; at an AOT concentration of $445 \mathrm{~g} / \mathrm{l}$ the deviation of the data is farthest from the $[\mathrm{P}]_{\text {aqf }}=[\mathrm{P}]_{\text {aq. } i}$ line, indicating greater surface activity.

Leodidis and Hatton [26] also showed that an interfacially active molecule can be identified through a plot of molecule transfer (in their case amino acid) ( $\left.t_{a}\right)$ versus the fractional water transfer $\left(t_{w}\right)$ to the organic phase. Simple mass balances show that $t_{a}$ and $t_{w}$ are given by: 


$$
t_{w}=1-\frac{V_{a q . f}}{V_{a q . i}}
$$

and

$$
t_{a}=1-\frac{[P]_{a q . f} V_{a q . f}}{[P]_{a q . i} V_{a q . i}}
$$

$t_{w}$ can be varied by changing the $\mathrm{pH}$ or the salt content of the aqueous phase or the AOT concentration in the initial organic phase. The penicillin transfer, $t_{a}$, is plotted against the fractional water transfer, $t_{w}$, on Figure 6 , which gives further support to penicillin being interfacially active in the presence of AOT. $t_{w}$ indicates the increase in organic phase volume; when $t_{w}$ is finite $V_{a q . f}<V_{a q . i}$ equations (7) and (8) give, when $t_{a}>t_{w}$

$$
t_{a}-t_{w}=\left(1-t_{w}\right)\left(1-\frac{[P]_{a q . f}}{[P]_{a q . i}}\right)
$$

and it is a requirement that $[P]_{\text {aq. }}<[P]_{\text {aq.i. }}$. The vertical displacement of the experimental data from the $t_{a}=t_{w}$ line is a measure of the strength of interfacial association; Figure 6 shows that penicillin $\mathrm{G}$ is slightly more interfacially active at surfactant concentrations of $267 \mathrm{~g} / \mathrm{l}$ and $88 \mathrm{~g} / \mathrm{l}$ than surfactant concentrations $445 \mathrm{~g} / \mathrm{l}$ and $333 \mathrm{~g} / \mathrm{l}$, and also that interfacial association is dependent on both $\mathrm{pH}$ and surfactant concentration. Similar trends were observed for the AOT-amino acid system by Leodidis and Hatton [26].

\section{Partitioning of penicillin $G$ between the organic and aqueous phases}

Penicillin G solubilisation into the reverse micellar phase is influenced by parameters that are related to the organic phase such as surfactant concentration, in addition to the properties of the aqueous phase (such as $\mathrm{pH}$ and salt concentration). The motivation for using AOT as the surfactant in this study was due to its ease of forming reverse micelles, its stability in comparison with other surfactants, and that it has been used in many published studies of other systems with success $[19,28]$. Initially, the effect of AOT concentration on reverse micellar extraction was examined using an aqueous solution with $\mathrm{pH} 7.60$ and $0.1 \mathrm{M} \mathrm{KCl}$. A 'white precipitate' was observed at the interface of some samples when the surfactant concentration used was less than $0.1 \mathrm{M}$; later experiments showed that the onset of precipitate formation was dependent on the ratio of penicillin concentration $[P]_{a q}$ to surfactant concentration [S]. Some researchers extracting proteins found that using AOT concentrations greater than $0.1 \mathrm{M}$ caused interfacial protein precipitation [29]. The 'white precipitate' is a dispersion phenomenon that occurred when the ratio $[P]_{a q}[S]$ was high because of low hydrophobicity in the system due to the low surfactant concentration used, which appears to be similar to protein aggregation rather than protein solubilisation into the organic phase found by others [11].

A sought after effect of the AOT concentration on penicillin extraction is to increase the amount of penicillin transferred to the reverse micellar phase. It was known that 
the surfactant concentration controls the number of reverse micelles and hence the capacity to solubilise biomolecules into the reverse micelle pool $[19,30]$. Increasing the surfactant concentration increases the number of reverse micelles [31], thus leading to improvement in extraction capacity of the reverse micelles. Figure 7 shows that as the concentration of AOT was increased from 0 to $222 \mathrm{~g} / 1$, the amount of penicillin extracted into the reverse micellar phase increased. However, increasing AOT concentration from $222 \mathrm{~g} / \mathrm{l}$ to $445 \mathrm{~g} / \mathrm{l}$ the penicillin $\mathrm{G}$ in reverse micelles decreases. There is an optimum surfactant concentration of between 100 and $200 \mathrm{~g} / \mathrm{l}$ that corresponds to the maximum limit for penicillin $\mathrm{G}$ transfer into the reverse micelles. Penicillin $\mathrm{G}$ is a small biomolecule (molecular weight of 356 $\mathrm{g} /$ mole); according to Pires $e$ t al. (30), small molecules are more easily extracted to the reverse micelles using low surfactant concentration. Moreover, our experiments showed that use of AOT concentration $>445 \mathrm{~g} / \mathrm{l}$ produced a stable emulsion (when subjected to gravity settling), making it difficult to separate and analyse.

It was found that penicillin $\mathrm{G}$ did not dissolve in the organic phase without AOT. Since penicillin $G$ was extracted into the organic phase only when reverse micelles were present, it is assumed that the penicillin $\mathrm{G}$ is located within their structure. The partition (distribution) coefficient, $K$, is defined by:

$$
K=\frac{[P]_{r m+o . f}}{[P]_{a q . f}}
$$

A higher value of the partition coefficient indicates better solubilisation of penicillin $\mathrm{G}$ into the reverse micelles.

Figure 8 shows that the distribution coefficient increases with increasing AOT concentration. The extent of solubilisation of penicillin into the reverse micellar phase depends on the quantity of reverse micelles, which during mixing are better dispersed in the bulk aqueous phase when more surfactant molecules are present. Haghtalab and Osfouri [20] asserted that adding more surfactant to the solution intensifies the interaction between the reverse micelles and the protein molecules (in their case) and result in the number of moles, and the maximum mole number of protein on the surface of the reverse micelles, being changed. Figure 8 shows that there is no saturation limit for the concentration of penicillin that can be transferred into the reverse micellar phase in the region $0<[P]_{a q . f}<7 \mathrm{~g} / \mathrm{l}$. However, there is a concentration limit of AOT which can solubilise maximum concentration of penicillin $\mathrm{G}$ into reverse micelles. The partition coefficients of penicillin $\mathrm{G}$ are effectively the same at $[S]=267$ and $445 \mathrm{~g} / \mathrm{l}$. This trend confirmed that penicillin $\mathrm{G}$ is an interfacially active compound which can easily transfer to reverse micelles.

It was found that the partition coefficients increase by using an higher initial concentration of penicillin in the feed (aqueous) phase appears to promote the penicillin solubilisation into the reverse micellar phase. A possible explanation is that at higher initial concentrations of penicillin in the aqueous phase the ion-dipole interaction between penicillin and AOT molecules are larger than ion-ion interaction between penicillin molecules.

In previous studies of protein and amino acid extraction using reversed micellar systems other researchers buffered the aqueous phase to adjust the $\mathrm{pH}[26,32,33]$. 
However, Wang et al. [4] pointed out that the buffers can interfere the solubilisation of biomolecules into the reverse micellar phase because the buffer anions may compete with the amino acid (in their case) to interact with the surfactant headgroup; in other work buffers were avoided because over such a wide range of $\mathrm{pH}$ values they would give the addition of an unnecessary mixture of ions to the system [34]. In this paper we have used $\mathrm{NaOH}$ and $\mathrm{HCl}$ to adjust the $\mathrm{pH}$. To examine the effect of $\mathrm{pH}$ on the partitioning of penicillin, aqueous $\mathrm{KCl}$ solutions containing $3.6 \mathrm{~g} / \mathrm{l}$ of penicillin and various $\mathrm{pH}$ values were contacted with solutions of AOT of various concentrations at volume ratios of 1:1. The results are shown in Figure 9 as a relation between the concentration of penicillin $\mathrm{G}$ in the organic phase and the $\mathrm{pH}$ value at the equilibrium state.

From Figure 9, it is clear that for all concentrations of AOT, the results obtained show a maximum penicillin $\mathrm{G}$ concentration in the reverse micellar phase at low $\mathrm{pH}$ values could be attributed to the high electrostatic attraction between AOT anions and the positively charged penicillin $\mathrm{G}$ aggregates at $\mathrm{pH} \approx 1$ to 2 .

Previous studies on protein extraction have also shown that $\mathrm{pH}$ plays important role in reverse micellar systems [8, 12, 30, 31, 35-38]. Noble and Varley [39], using protein as the biomolecule, pointed out that the $\mathrm{pH}$ of the protein and surfactant solutions could be manipulated so that the protein would exhibit a net charge opposite to that of the surfactant, thus allowing electrostatic interactions between the protein and surfactant in the different phases. Kilikian et al. [37] explained that he overall protein charge is determined by the $\mathrm{pH}$ of the aqueous phase and the isoelectric point ( $\mathrm{pI}$ ) of the biomolecule used (protein, in their case). If the $\mathrm{pH}$ of the aqueous phase is higher than the $\mathrm{pI}$, the charge is negative, but if the $\mathrm{pH}$ is lower than the pI, the charge is positive. Using this postulate for the anionic AOT, the penicillin $\mathrm{G}(\mathrm{pI}=2.74)$ is expected to have strong attraction with the surfactant head group at $\mathrm{pH}<2.74$. Hence, more penicillin $\mathrm{G}$ could be solubilized into the reverse micellar solution at $\mathrm{pH}<\mathrm{pI}$. From the viewpoint of industrial production of penicillin $\mathrm{G}$, extraction is carried out at $\mathrm{pH} 2.5-3.0$. Figure 9 shows that at $\mathrm{pH}>\mathrm{pI}$ a higher AOT concentration needs to be used in order to get the same concentration of penicillin $\mathrm{G}$ in the organic phase as at $\mathrm{pH}<\mathrm{pI}$; however, one should take into account that there is an optimum AOT concentration $(222 \mathrm{~g} / \mathrm{l})$. The concentration of penicillin $\mathrm{G}$ in reverse micelles decreases dramatically when $[S]=445 \mathrm{~g} / \mathrm{l}$ is used. This raises the question of whether the $\mathrm{pH}$ can be manipulated to get better penicillin $\mathrm{G}$ extraction at low AOT concentration in an industrial application (it should be noted that penicillin broths are compositionally more complex than those used in this work, which will change the equilibria that can established in the extraction process). The use of a low surfactant concentration would be helpful because amphiphilic molecules are difficult to remove in subsequent downstream processing [18].

Ono et al. [8], using anionic surfactant DOLPA to extract hemoglobin ( $\mathrm{pI}=6.8$ ) found that even though the best extraction was obtained at $\mathrm{pH}<\mathrm{pI}(5.5-6.5)$, a precipitate occurred at $\mathrm{pH}<5$. They found that at the $\mathrm{pH}<<\mathrm{pI}$, the biomolecules tend to form a complex that binds to the surfactant. In this study, we observed precipitation when high initial penicillin $\mathrm{G}$ concentrations were used at low $\mathrm{pH}$. Although an extremely low $\mathrm{pH}$ range was used, at $[\mathrm{KCl}]=10 \mathrm{~g} / \mathrm{l}$ and $400 \mathrm{rpm}$ stirring speed, a transparent two phase system was obtained with a clear interphase when $[P]_{\text {aq. } i}<10 \mathrm{~g} / \mathrm{l}$. From the results it appears that the $\mathrm{pH}$ effect is important for 
the penicillin $\mathrm{G}$ extraction, although the electrostatic contribution is also relevant for the AOT reverse micelles which achieved better binding at higher $\mathrm{pH}$ values.

Whilst some of the thermodynamic models for solubilisation suggest that there should be an effect of $\mathrm{pH}$ (for example, equation (5)), others do not (see equation (4)). In this work the dependence of the partition coefficient (see equation (10)) on penicillin concentration, AOT concentration, $\mathrm{pH}$, and salt type and concentration has been measured. When the partition coefficient, $K$, is plotted against the concentration of penicillin in the aqueous phase, $[P]_{a q . f}$, (all other parameters being held constant) and the experimental data extrapolated back to $[P]_{\text {aq. }}=0$ line, a value for the partition coefficient at infinite dilution, $K^{\infty}$, is found. Following this procedure, the results for $\mathrm{pH} 1.65$ and 7.6 are shown in Figure 10.

The distribution coefficient at infinite dilution depends markedly on $\mathrm{pH}$ and surfactant concentration. $K^{\infty}$ increases as the surfactant concentration increases, and is is higher at $\mathrm{pH} 1.65$. However, one should notice that the limitation of using a pH as low as $\mathbf{1 . 6 5}$ is that the extraction becomes troublesome due to formation of a precipitate except at very low penicillin concentrations. So whilst there is clearly a $\mathrm{pH}$ effect on $K^{\infty}$, factors have to be taken into account in a practical sense.

\section{Effects of salt type and concentration}

The salt concentration of the aqueous phase influences the solubilisation of penicillin into the reverse micellar phase. The salt concentration was investigated by varying the $\mathrm{KCl}$ concentration in the aqueous phase at $\mathrm{pH} 7.6$. When $[\mathrm{KCl}]>10 \mathrm{~g} / \mathrm{l}$ the penicillin concentration in the organic phase was found to decrease, although increasing $[\mathrm{KCl}]$ from 0 to $10 \mathrm{~g} / \mathrm{l}$ showed a significant increase of penicillin in the organic phase. A decrease of the water concentration in the micellar phase was found to accompany an increase of $[\mathrm{KCl}]$.

At $[\mathrm{KCl}]<5 \mathrm{~g} / \mathrm{l}$ almost all of the surfactant migrated to the aqueous phase and no reverse micelles were formed in the organic phase. This phenomenon was observed visually, and made addition of salt to the aqueous penicillin $\mathrm{G}$ solution very important to avoid the formation of a stable emulsion. A minimum amount of salt is necessary to salt out the surfactant from the excess aqueous phase into the organic phase to form reverse micelles $[40,41]$. Reverse micelles will not form if there is too little salt in the aqueous phase; however, if there is too much salt, the efficiency of surfactant molecules to solubilise the penicillin $\mathrm{G}$ molecules reduces. Wang et al. [4] reported that ionic strength is required to reduce electrostatic repulsion between the surfactant head groups, and reported that if no salt is added to the system the aggregation of surfactant and/or the surfactant and protein complex formed a coagulum and induced a phase separation problem. The solubilisation of water by an AOT reverse micellar solution in equilibrium with an aqueous phase (in both cases with and without penicillin $G$ ) was found to be a strong function of salt concentration in our experiments. Others have suggested that decreasing both biomolecule concentration and water content in the organic phase happens because of a size exclusion effect occurring with the decrease in micelle size [42, 43], asserting that a decrease in the solubilisation of the enzyme with increases in ionic strength resulted from smaller micelles being formed because of the reduced interaction between the surfactant polar head group and the hydrophilic region of the enzyme. 
Our experimental results showed that penicillin $\mathrm{G}$ could be extracted into the reverse micellar phase quantitatively over a relatively small salt concentration range. With increasing salt concentration over $10 \mathrm{~g} / \mathrm{l}$, the extent of penicillin $\mathrm{G}$ transfer decreased in spite of the $\mathrm{pH}$ being in the region where the electrostatic interaction between antibiotic and surfactant is attractive. According to Dekker et al. [29] the ionic strength of the aqueous phase determines the degree of shielding of the electrostatic potential imposed by a charged surfactant head group. They also suggested that by increasing the ionic strength the electrostatic interaction between the charged penicillin molecules and the charged surfactant head groups is reduced.

Salt type also plays an important role in the extraction of biomolecule from the aqueous to the reverse micellar phases $[10,44-46]$. We used two salts, $\mathrm{KCl}$ and $\mathrm{CaCl}_{2}$, dissolved in the aqueous phase and found that the concentration of penicillin $\mathrm{G}$ in the reverse micellar phase is higher when using $\mathrm{CaCl}_{2}$. This is because a divalent salt like $\mathrm{CaCl}_{2}$ leads to smaller reverse micelle droplets and the ability to solubilise more penicillin is increased. However, it was observed that when using $\mathrm{CaCl}_{2}$ the solution became more sensitive to the stirring speed and tended to emulsify more easily. This caused problems with getting a good phase separation, and even when the phases can be separated extra time was needed for the solutions to achieve equilibrium.

\section{Reverse micelle formation and water content}

The water content in the AOT/isooctane organic phase was measured using a Karl Fischer titration method after mixing with two $0.1 \mathrm{M} \mathrm{KCl}$ solutions, one with and one without penicillin $\mathrm{G}$, in order to examine the formation of reverse micelles. Figure 11 shows the water content in the organic phase before and after the mixing process. The mixing process increased the water content in the reverse micellar phase (due to the presence of the AOT). When using the same AOT concentration but without mixing there was no solubilisation of water into the organic phase. This shows that the AOT carries water to the organic phase and form reverse micelles, and that mixing is an important process to produce solubilisation of water into the organic phase. After the extraction process was accomplished, the organic phase was clear and transparent indicating that the formation of AOT reverse micelles had been achieved. It also shows that the reverse micelles transfer a large amount of water to the organic solution, and since the penicillin molecules solubilise in water but not in isooctane the reverse micelle system has the ability to extract penicillin molecules into the organic solution.

Figure 11 confirms the above and shows that the water content in the organic phase increases in proportion to the AOT concentration, and without surfactant $([S]=0)$ there is no water uptake. The water content values level off when $[S] \approx 450 \mathrm{~g} / 1$ indicating that the amount of water that can be carried to the reverse micellar phase by the AOT molecules is limited. At $[S]>667 \mathrm{~g} / \mathrm{l}$ the whole solution became a stable emulsion, making it is impossible to separate the penicillin molecules from the initial aqueous phase since no phase separation occur after the mixing process.

The water content of the micellar phase is an important parameter affecting penicillin $\mathrm{G}$ extraction into a reverse micellar system. Previous studies have shown that water content has a strong influence on protein solubilisation and subsequent functions 
such as the size of reverse micelles $[8,37,47]$. Those researchers used the molar ratio of water to surfactant in the micellar organic phase $\left(W_{o}\right)$ as an important parameter in understanding what affects the characteristics of the reverse micelles, such as their size. The water content analysis is also important in order to determine the structure of reverse micelles and the number of surfactant molecules per reverse micelle [47]. To examine the relationship between the water content in the organic phase and penicillin transfer, an experiment was carried out to measure the water content in the organic phase in the extraction system with and without penicillin. The water content in the system without penicillin was found to be higher than that with penicillin. A similar phenomenon was observed by Ono et al. [8] who used DOLPA reverse micelles to extract haemoglobin. They suggested that water never accompanied the haemoglobin molecules when they were dissolved into the reverse micelles, because haemoglobin extraction consumes the surfactants to form empty reverse micelles. In our penicillin $\mathrm{G}$ experiments, to fill the reverse micelles' pools in the organic phase the system without penicillin encouraged more reverse micelles containing water compared to the system containing penicillin.

The effect of the AOT concentration and the initial amount of penicillin $\mathrm{G}$ on the solubilising water was also investigated with experiments carried out at $\mathrm{pH} 7.6$ and $0.1 \mathrm{M} \mathrm{KCl}$ (there was no precipitation or emulsion observed in these experiments). The transparent interface formed, with no insoluble aggregates, indicated that the reverse micelles solubilised successfully in the organic phase. An increase in surfactant concentration from $267 \mathrm{~g} / \mathrm{l}$ to $445 \mathrm{~g} / \mathrm{l}$ caused the water uptake to increase from $25 \%$ to $35 \%$. These results agree with studies of protein extraction $[9,48,49]$. For example, Spirovska and Chaudhuri [50] showed that the percentage content of water in the organic phase increased proportionally with the surfactant concentration due to more reverse micelle molecules being solubilised in the organic phase.

To determine a reverse micelle size, other researchers have recommended equations which relate the molar ratio of water to surfactant $\left(W_{o}\right)$ values. From purely geometrical considerations and assuming the micelles to be monosized with all the surfactant located in a monolayer at the aqueous-organic interface, the reverse micelle radius (water pool radius), $r_{r m}$, is related to $W_{o}$ by [52]

$$
r_{r m}=\left(\frac{3 V_{H_{2} O}}{a_{A O T}}\right) W_{o}
$$

where $V_{H 2 O}$ is the volume of a water molecule (approximately $0.03 \mathrm{~nm}^{3}$ ) and $a_{A O T}$ is the surface area of the surfactant head group $\left(0.5 \mathrm{~nm}^{2}\right)$.

For $[\mathrm{S}]>20 \mathrm{~g} / \mathrm{l}$, the experimental values of $W_{o}$ was fairly constant and found to be in the range $15.7 \leq W_{o} \leq 17.6$ and the calculated reverse micelles radii were around 3 $\mathrm{nm}$. It has been reported that with the increase of surfactant concentration, the micellar size does not change for most surfactants, which is the case for AOT [52]. The results from our work are in agreement with the results of previous works which suggest that the calculated value of $r_{r m}$ varies between 1 and $3 \mathrm{~nm}$. Using the same assumptions as lie behind equation (11), and further assuming that the projected areas of the AOT head groups are packed at the micelle surface with a porosity of 0.4 , 
the number of AOT molecules per reverse micelle is about 360 . This lies well within the range $100-900$ reported by Lye [52].

Many studies have shown that other parameters such as $\mathrm{pH}$ and salt concentration can affect the solubilisation of biomolecules into the reverse micelle phase; however, the results from the penicillin $\mathrm{G}$ extraction study here show that surfactant concentration is the most important parameter affecting the solubilisation of penicillin into the reverse micelle phase. Ichikawa et al. [9], extracting protein using the AOT reverse micelle system, found that even using suitable $\mathrm{pH}$ and sait concentration the extraction will not happen if insufficient AOT concentration is used. They suggested that the solubilising water provides the hydrophilic surroundings that are vital in the reverse micelle extraction process, and that the AOT molecules seemed to take part of the water solubilising agent in the process. Similar to the results from this study, they also found that the maximum water solubilisation increased with increasing surfactant concentration.

\section{CONCLUSIONS}

Experiments on the solubilisation of penicillin $G$ into reverse micelles of AOT have been carried out. The results show that the concentration of penicillin $\mathrm{G}$ in the organic phase depends primarily on the $\mathrm{pH}$ and surfactant concentration, with the salt type and concentration in the aqueous phase also being important. However, the effect of the salt type and concentration is sufficiently great to signify that extraction from a broth needs to be assessed experimentally.

The results show that penicillin $\mathrm{G}$ is an interfacially active compound that interacts with AOT, with the interfacial association being dependent on both $\mathrm{pH}$ and surfactant concentration. When the concentration ratio $[P]_{a q} /[S]$ is high, precipitation of the penicillin occurs, which limits the possible use of reverse micellar extraction at low values of $[P]_{a q}$.

The distribution coefficient favours solubilisation of the penicillin into the reverse micelles at moderate AOT concentrations. There is no saturation limit of penicillin $G$ transferred into reverse micelles; there is a limit of surfactant concentration beyond which the concentration of penicillin $G$ transferred into reverse micelles remains constant. The distribution coefficient at infinite dilution, $K^{\infty}$, has been shown to be a function of both $\mathrm{pH}$ and surfactant concentration. Similar trends in the value of $K^{\infty}$ were observed at different $\mathrm{pH}$ values; $K^{\infty}$ increases as the surfactant concentration is increased. However, although $K^{\infty}$ is higher at $\mathrm{pH} 1.65$ the extraction is difficult due to precipitation when high initial penicillin concentration is used.

Reverse micelle formation affects the volume of water transferred into the organic phase (this water is held within the reverse micelles). From measurements of the water transferred, the size of the reverse micelles was estimated to be about $3 \mathrm{~nm}$ and the number of AOT molecules per reverse micelle about 360 . These values are in broad agreement with previous work using AOT to extract proteins.

\section{NOMENCLATURE}




$\begin{array}{ll}a_{A O T} & \text { area of a surfactant head group } \\ \text { AOT } & \text { bis(2-ethylhexyl) sulfosuccinate } \\ \text { DOLPA } & \begin{array}{l}\text { dioleyl phosphoric acid } \\ \text { distribution coefficient }\end{array} \\ K^{\infty} & \text { distribution coefficient at infinite dilution } \\ {[K C l]} & \mathrm{KCl} \text { concentration } \\ {[P]_{a q . f}} & \text { final concentration of penicillin } \mathrm{G} \text { in the aqueous phase } \\ {[P]_{a q . i}} & \text { initial concentration of penicillin } \mathrm{G} \text { in the aqueous phase } \\ {[P]_{r m+o . f}} & \text { final concentration of penicillin } \mathrm{G} \text { in the reverse micelle organic phase } \\ r_{r m} & \text { reverse micelle radius } \\ {[S]} & \text { surfactant concentration } \\ t_{p} & \text { fractional transfer of penicillin } \mathrm{G} \\ t_{w} & \text { fractional water transfer } \\ V_{a q . f} & \text { final volume of aqueous phase } \\ V_{a q . i} & \text { initial volume of aqueous phase } \\ V_{H 2 O} & \text { volume of a water molecule } \\ W_{o} & \text { molar ratio of surfactant concentration to water concentration } \\ \theta_{1} & \text { fractional coverage according to a Langmuir type isotherm } \\ \Lambda_{i j} & \text { Wilson parameters }\end{array}$

\section{ACKNOWLEDGEMENTS}

The authors would like to acknowledge the financial support from the Universiti Teknologi Malaysia for the scholarship awarded to Siti Hamidah Mohd Setapar to make this study possible.

\section{REFERENCES}

[1] Yang, C. and Cussler, E. L., 2000 , Reactive extraction of penicillin G in hollowfiber and hollow-fiber fabric modules. Biotechnol. Bioeng. 691: 66-73.

[2] Soto, A., Arce, A. and Khoshkbarchi, M. K., 2005, Partitioning of antibiotics in a two-liquid phase system formed by water and a room temperature ionic liquid. Sep. Purif. Tech. 44: 242-246.

[3] Nabais, A.M.A and Cardoso, J.P., 1999, Purification of benzylpenicillin filtered broths by ultrafiltration and effect on solvent extraction. Bioproc. Biosys. Eng. 21: 157-163.

[4] Wang, W., Weber, M. E. and Vera, J. H., 1995, Reverse Micellar Extraction of Amino Acids Using Dioctydimethylammonium Chloride. Ind. Eng. Chem. Res. 34: 599-606.

[5] Tessier, L., Bouchard, P. and Rahni, M., 2005, Separation and purification of benzylpenicillin produced by fermentation using coupled ultrafiltration and nanofiltration technologies. J. Biotech. 116: 79-89.

[6] Hano, T., Michiaki, M. and Takaaki, O., 1994, Continuous extraction of penicillin $\mathrm{G}$ with liquid surfactant membrane using Vibro Mixer ${ }^{\circledR}$. J. Membrane Sci. 93: 61-68

[7] Naoe, K., Noda, K., Kawagoe, M. and Imai, M., 2004, Higher order structure of proteins solubilized in AOT reverse micelles. Coll. Surf. B. 38: 179-185.

[8] Ono, T., Goto, M., Nakashio, F. and Hatton, T. A., 1996, Extraction behaviour of haemoglobin using reversed micelles by diolyel phosphoric acid. Biotechnol. Prog. 12: 793-800. 
[9] Ichikawa, S., Imai, M. and Shimizu, M., 1992, Solubilizing water involved in protein extraction using reversed micelles. Biotechnol. Bioeng. 39: 20-26.

[10] Kinugasa, T., Kondo, A., Mouri, E., Ichikawa, S., Nakagawa, S., Nishii, Y., Watanabe, K. and Takeuchi, H., 2003, Effects of ion species in aqueous phase on protein extraction into reversed micellar solution. Sep.Purif. Tech. 31: 251-259.

[11] Kyung, H. N. and Jee, Y. I., 2005, One-step separation of lysozyme by reverse micelles formed by the cationic surfactant, cetyldimethylammonium bromide. Food Chem. 93 (1): 95-101.

[12] Hu, Z. and Gulari, E., 1996, Extraction of aminoglycoside antibiotics with reverse micelles. J. Chem. Technol. Biotechnol. 65: 45-48.

[13] Carlson, A. and Nagarajan, R., 1992, Release and recovery of porcine pepsin and bovine cymosin from reverse micelles: A new technique based on isopropyl alcohol addition. Biotechnol. Prog. 8: 85-90.

[14] Chang, Q. L., Chen, J. Y., Zhang, X. F. and Zhao, N. M., 1997, Effect of the cosolvent type on the extraction of $\alpha$-amylase with reversed micelles:circular dichroism study. Enz. Microb. Technol. 20: 87-92.

[15] Pileni M. P. Reverse micelles used as teplates: a new understanding in nanocrystal growth. J. Exp. Nanosci. 1(1): 13-27.

[16] Bong, K. L, Dong, P. H, Sung, S. L and Kuboi, R., 2004, Analysis of protein back-extraction processes in alcohol-and carboxylic acid-mediated AOT reverse micellar systems based on structural changes of proteins and reverse micelles. Biochem. Eng. J. 22: 71-79.

[17] Brandani, V., Giacomo, G. D. and Spera, L., 1996, Recovery of a-amylase extracted by reverse micelles. Proc. Biochem. 31(2): 125-128.

[18] Goto, M., Ishikawa, Y., Ono, T., Nakashio, F. and Hatton, T. A., 1998, Extraction and activity of chymotrypsin using AOT-DOLPA mixed reversed micellar systems. Biotechnol. Prog. 14: 729-734.

[19] Jun,G. L., Jian, M. X., Rui, S., Cheng, L. Y. and Hui, Z. L., 2004, Reverse micelles extraction of nattokinase from fermentation broth. Biochem. Eng. J. 21: 273-278.

[20] Haghtalab, A. and Osfouri, S., 2003, Vacancy solution theory for partitioning of protein in reverse-micellar system. Separation Science and Technology 38 (3): 553-569.

[21] Woll, J. M., Hatton, T. A. and Yarmush, M. L., 1989, Bioaffinity separations using reversed micellar extraction. Biotechnol. Prog. 5: 57-62.

[22] Rabie, H. R., Vera, J. H., 1998, A simple model for reverse micellear extraction of proteins. Sep. Sci. Technol. 33 (8): 1181-1193.

[23] Ashrafizadeh, S. N. and Khoshkbarchi, M. K., 1998, Modelling and experimental data for the reverse micellar extraction of proteins using a new surfactant. Sep. Sci. Technol. 33 (16): 2579-2595.

[24]Bruno, P., Caselli, M., Luisi, P. L., Maestro, M., Traini, A., 1990, A simplified thermodynamic model for protein uptake by reverse micelles: theoretical and experimental results. J. Phys. Chem. 94 (15) :5908-5917.

[25] Brandani, S., Brandani, V., Giacomo, G. D., 1994, A thermodynamic model for protein partitioning in reverse micellar systems. Chem. Eng. Sci. 49 (21): 36813686.

[26] Leodidis, E. B. and Hatton, T. A., 1990, Amino acids in AOT reversed micelles. 1. Determination of interfacial partition coefficient using the phase-transfer method. J. Phys. Chem. 94: 6400-6411. 
[27] Woll J. M. and Hatton T. A., 1989, A simple phenomenological thermodynamic model for protein partitioning in reversed micellar systems. Bioprocess Eng. 4: 193-199.

[28] Naoe, K., Nishino, M., Ohsa, T., KAwagoe, M. and Imai, M., 1999, Protein extraction using sugar ester reverse micelles. J. Chem. Technol. Biotechnol. 74: 221-226.

[29] Dekker, M., Van't Riet, K., Bijsterbosch, B. H., Wolbert, R. B. G. and Hilhorst, R., 1989, Modelling and optimization of the reversed micellar extraction of aamylase. AlChE J. 35- 321.

[30] Pires, M. J., Aires-Barros, M. R. and Cabral, J. M. S., 1996, Liquid-liquid extraction of proteins with reverse micelles. Biotechnol. Prog. 12: 290-301.

[31] Yu, C. Y., Chu, Y. and Ji, J. Y., 2003, Study of the factors affecting the forward and back extraction of yeast-lipase and its activity by reverse micelles. $J$. of Colloid and Interface Sci. 267: 60-64.

[32] Goklen, K. and Hatton, T. A., 1987, Liquid-liquid extraction of low molecular weight proteins by selective solubilization in reverse micelles. Sep. Sci. Technol. 22: 831-841.

[33] Jolivalt, C., Minier, M. and Renon, H., 1993, Extraction of cytochrome $c$ in sodium dodecylbenzenesulfonate microemulsions. Biotechnol. Prog. 9: 456-461.

[34] Andrews, B. A., 1993, The effect of protein characteristics on their extraction in reversed micelle systems. Sep. Biotech. 3: 29-36.

[35] Tzeng, Y. M., Tsun, H. Y and Chang, Y. N., 1999, Recovery of thuringiensin with cetylpyridinium chloride using micellar-enhanced ultrafiltration process. Biotechnol. Prog. 15: 580-586.

[36] Nishiki, T., Sato, I., Katoaka, T. and Kato, D., 1993, Partitioning behaviour and enrichment of proteins with reversed micellar extraction:1. Forward extraction of proteins from aqueous to reversed micelle phase. Biotechnol. Bioeng. 42: 596-600.

[37] Kilikian, B. V., Bastazin, M. R., Minami, N. M., Goncalves, M. R. and Junior, A. P., 2000, Liquid-liquid extraction by reversed micelles in biotechnological processes. Braz. J. Chem. Eng. 17: 29-38.

[38] Hu, Z. and Gulari, E., 1996, Protein extraction using the sodium bis (2ethylhexhyl) phosphate (NaDEHP) reverse micellar system. Biotechnol. Bioeng. 50: 203-206.

[39] Noble, M. J. and Varley, J. Colloidal gas asprons generated from the anionic surfactant AOT for the separation of proteins from aqueous solution. J. Chem. Technol. Biotechnol. 74: 231-237.

[40] Rabie, H. R., Helou, D., Weber, M. E. and Vera, J. H., 1997, Comparison of the titration and contacts methods for the water solubilization capacity of AOT reverse micelles in the presence of a cosurfactant. J. Coll. Interface Sci. 189: 208215.

[41] Wang, W., Weber, M. E. and Vera, J. H., 1994, Effect of alcohol and salt on water uptake of reverse micelles formed by dioctyldimethyl ammonium chloride (DODMAC) in isooctane. J. Coll. Interface Sci. 168: 422-427.

[42] Krieger, N., Taipa, M. A.,Aires-Barros, M. R., Melo, E. H. M., Lima-Filhoc, J. L. and Cabral, J. M. S., 1997, Purification of the citrinum Lipase Penicillium using reversed micelles AOT. J. Chem. Tech. Biotechnol. 69: 77-85.

[43] Aires-Barros, M. R. and Cabral, J. M. S. Selective separation and purification of two lipases from chromobacterium viscosum using AOT reversed micelles. Biotech. Bioeng. 38:1302-1307. 
[44] Leodidis, E. B. and Hatton, T. A., 1989, Specific ion effects in electrical double layers: Selective solubilization of cations in Aerosol-OT reversed micelles.

Langmuir 5: 741-753.

[45] Cheng, H. and Sabatini D. A., 2001, Reverse-micellar extraction for micellarsolubilized contaminant and surfactant removal. Sep. Purif. Tech. 24: 437-449.

[46] Goto, M., Ono, T., Horiuchi, A. and Furusaki, S., 1999, Extraction of DNA by reversed micelles. J. Chem. Eng. Japan 32 (1): 123-125.

[47] Naoe, K., Murata, M., Ono, C., Kawagoe, M. and Imai, M., 2002, Efficacy of guanidium salts in protein recovery from reverse micellar organic media. Biochem. Eng. J. 10: 137-142.

[48] Frense, D., Hadtendorn, R. and Ulbrich-Hofmann, R., 1995, 2-Modified 1,3diacylglycerols as new surfactants for the formation of reverse micelles. Chem. Phys. Lipids. 78: 81-87.

[49] Kinugasa, T., Tanahashi, S. I and Takeuchi, H., 1991, Extraction of lysozyme using reverse micellar solution: Distribution and extraction rates. Ind. Eng. Chem. Res. 30: 2470-2476.

[50] Spirovska, G. and Chaudhuri, J. B., 1998, Sucrose enhances the recovery and activity of ribonuclease A during reversed micelle extraction. Biotechnol. Bioeng. 58 (4): 374-379.

[51] Zulauf, M. and Eicke, H. F., 1970, Inverted micelles and microemulsions in the ternary system $\mathrm{H}_{2} \mathrm{O}$ /aerosol-OT/isooctane as studied by photon correlation spectroscopy. J. Phys Chem. 83: 480-486.

[52] Lye, J. L., 1993, Kinetic studies on the extraction of proteins using reverse micelles. PhD Thesis. University of Reading, UK.

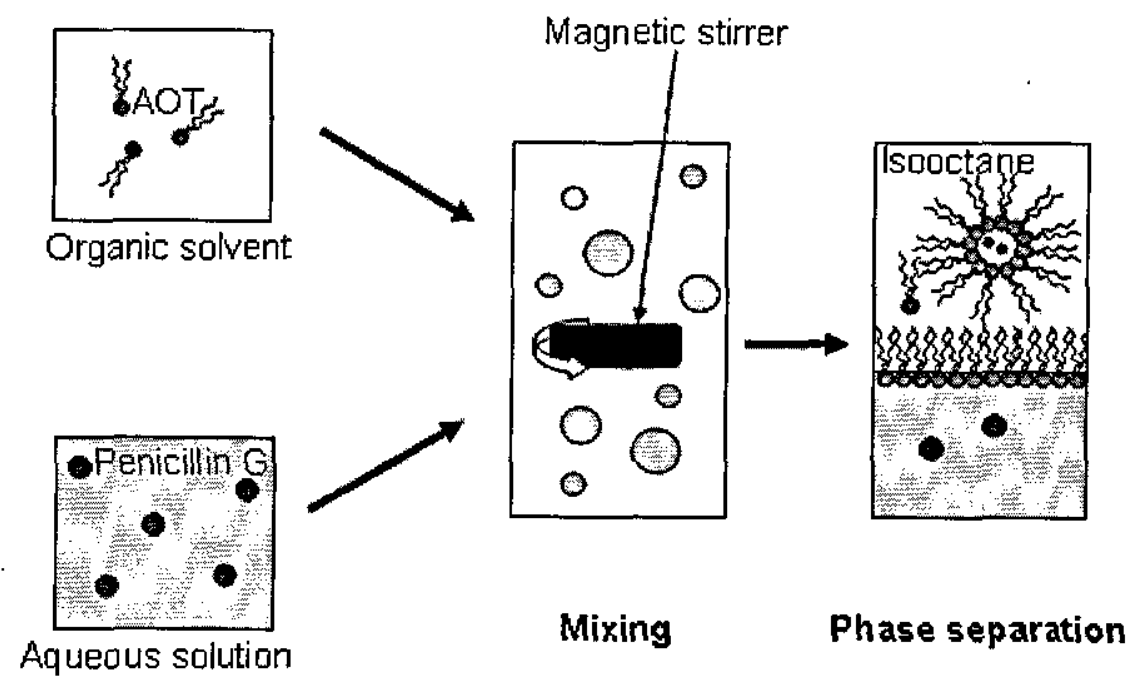

Figure 1 Diagram of the experimental procedure. Aqueous solution containing the penicillin to be extracted is contacted with an organic phase (isooctane) containing the surfactant, and then the phases are allowed to separate. 


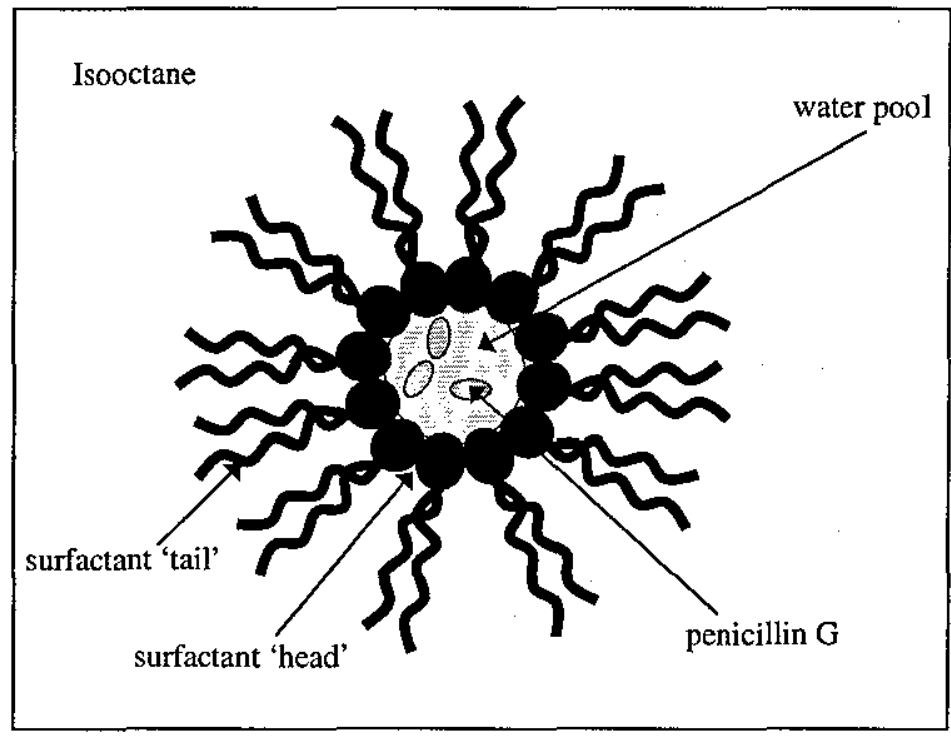

Figure 2. Schematic diagram of reverse micellar phase. The penicillin is entrapped in a water pool formed inside the reverse micelle, which itself lies in the organic (isooctane) continuous phase.

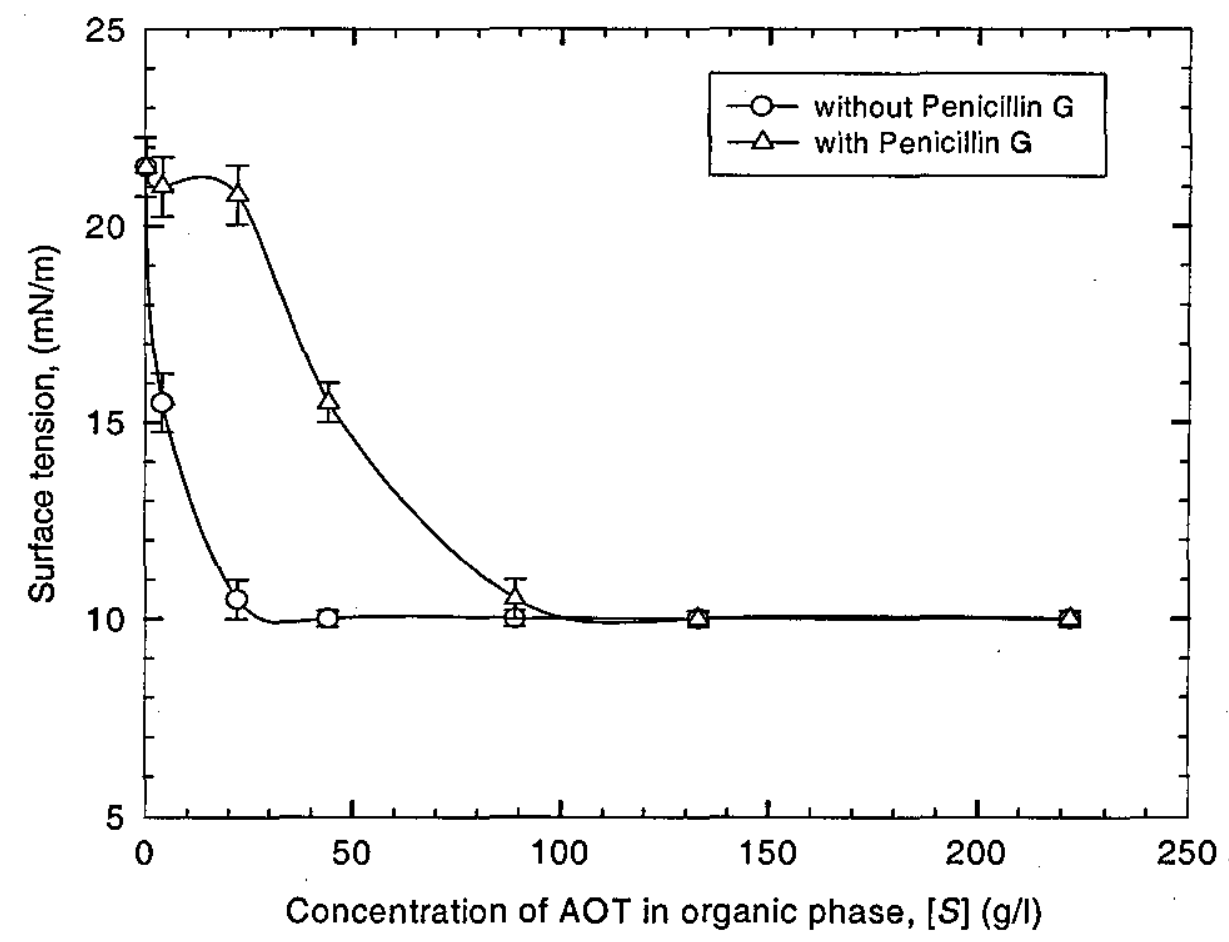

Figure 3. Effect of AOT concentration on the surface tension of isooctane with and without penicillin $\mathrm{G}$. (Initial penicillin in the aqueous phase, $\left.3.6 \mathrm{~g} \mathrm{l}^{-1} ; \mathrm{KCl}\right]=10 \mathrm{~g} \mathrm{l}^{-1}$; temperature, $23 \pm 1^{\circ} \mathrm{C}$; stirring speed, $400 \mathrm{rpm}$ for 10 minutes; $\mathrm{pH} 7.6$.) 


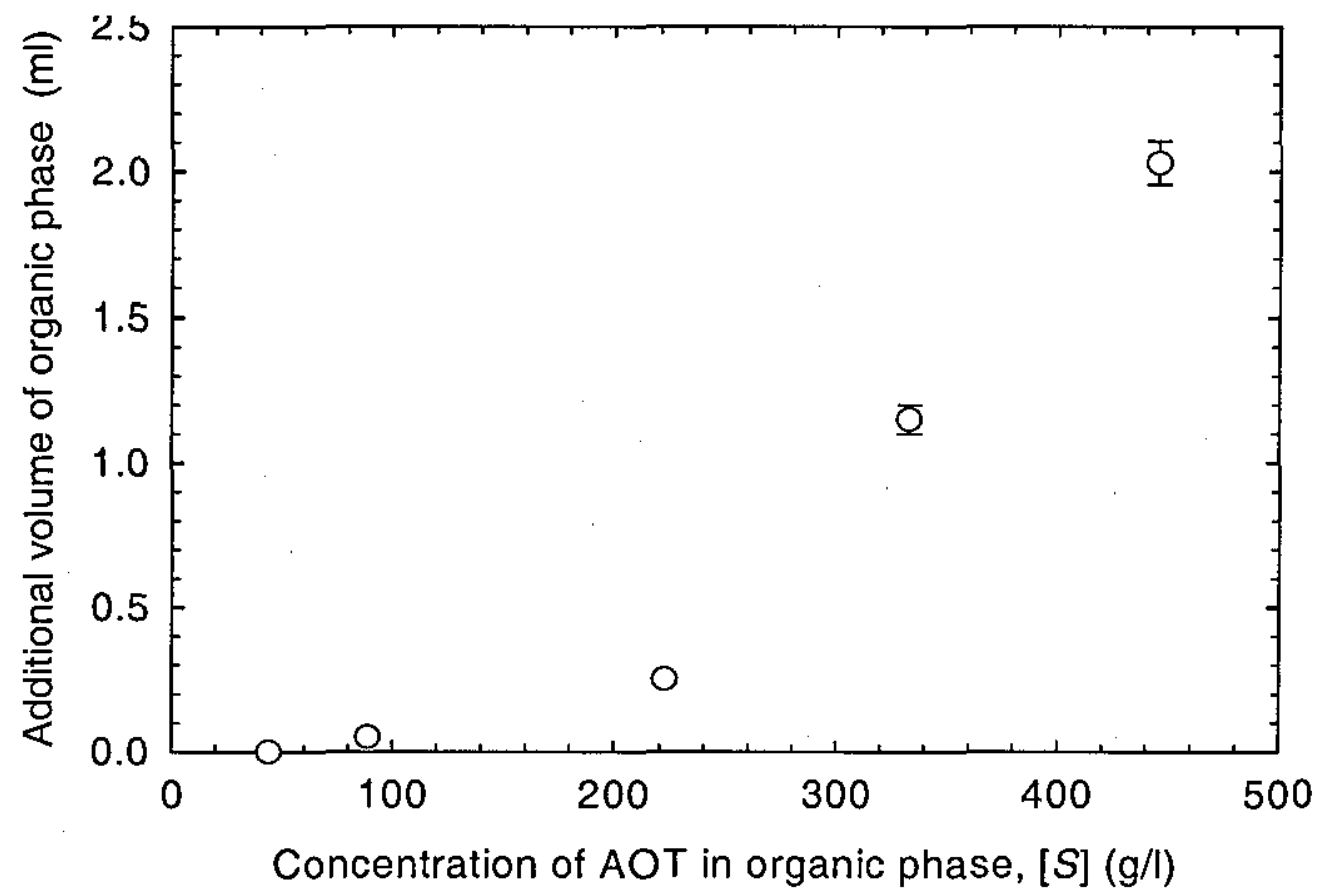

Figure 4. Additional volume of the organic phase at different AOT concentrations. (Initial volumes of the organic and aqueous phases were each $5 \mathrm{ml} ;[\mathrm{KCl}]=10 \mathrm{~g} / \mathrm{l}$; temperature, $23 \pm 1^{\circ} \mathrm{C}$; stirring speed, $400 \mathrm{rpm}(10 \mathrm{~min}) ; \mathrm{pH} 7.6$; initial penicillin $\mathrm{G}$ concentration in the aqueous phase, $3.6 \mathrm{~g} / \mathrm{l}$.)

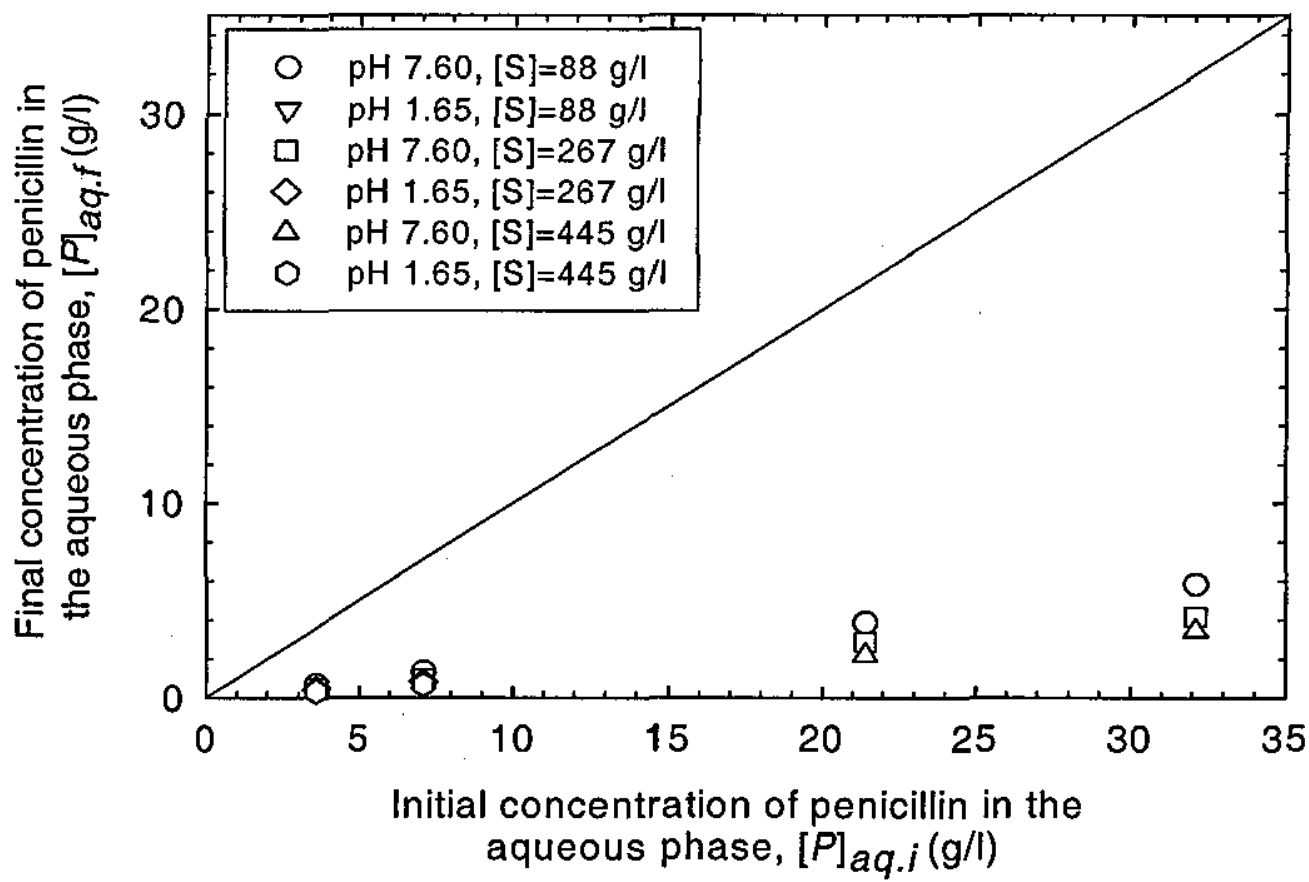

Figure 5. Final penicillin $\mathrm{G}$ concentration in the aqueous phase $\left([P]_{a q_{f} f}\right)$ plotted against the initial penicillin $\mathrm{G}$ concentration before extraction $\left([P]_{a q . i}\right)$ at $23^{\circ} \mathrm{C}$ and

$\mathrm{KCl} 10 \mathrm{~g} / \mathrm{l}$. Deviations from the $[P]_{a q . f}=[P]_{\text {aq. } i}$ line indicate that the penicillin molecule is interfacially active. 


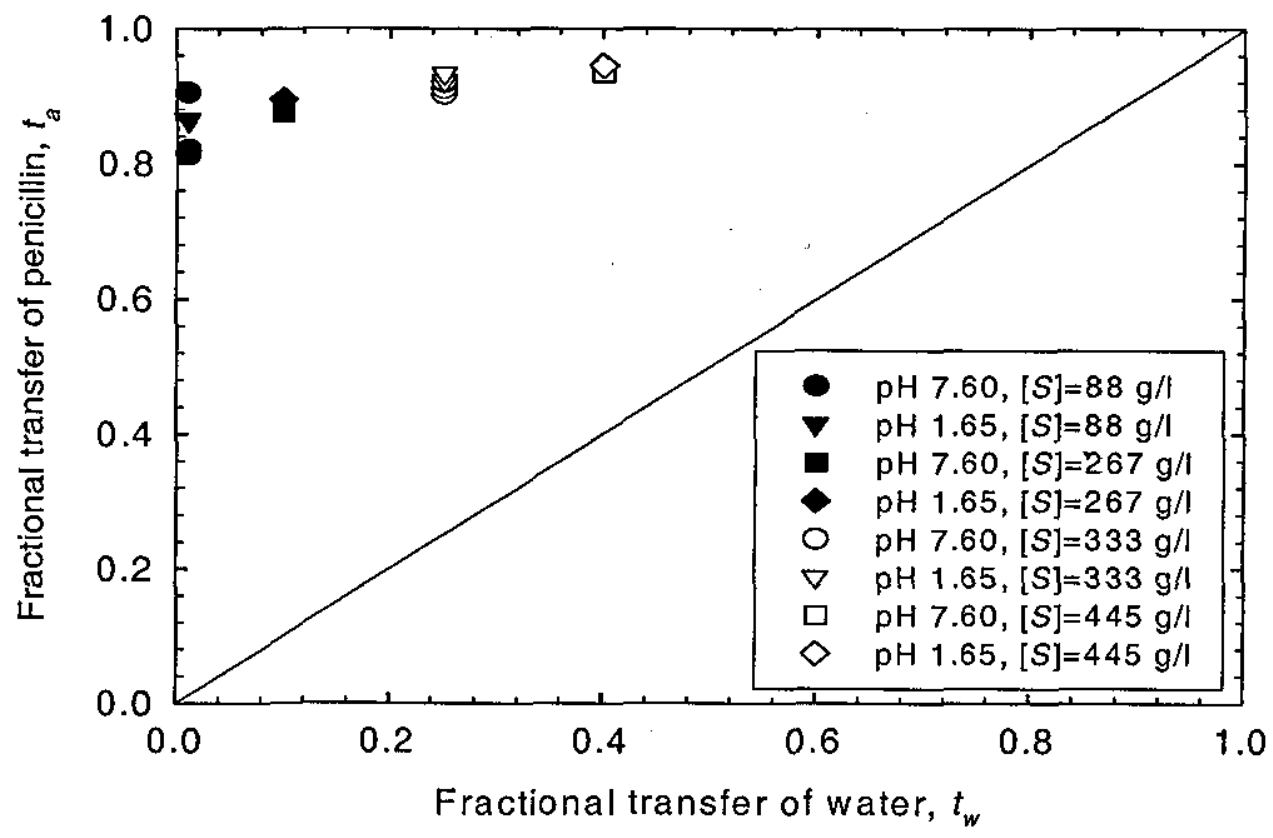

Figure 6 Fractional transfer of penicillin $\mathrm{G}\left(t_{a}\right)$ plotted against the fractional water transfer $\left(t_{w}\right)$ to the reverse micelle organic phase at different $\mathrm{pH}$ values and AOT concentrations. Deviations from the $t_{w}=t_{a}$ line are indicative that penicillin $\mathrm{G}$ is interfacially active in the presence of AOT.

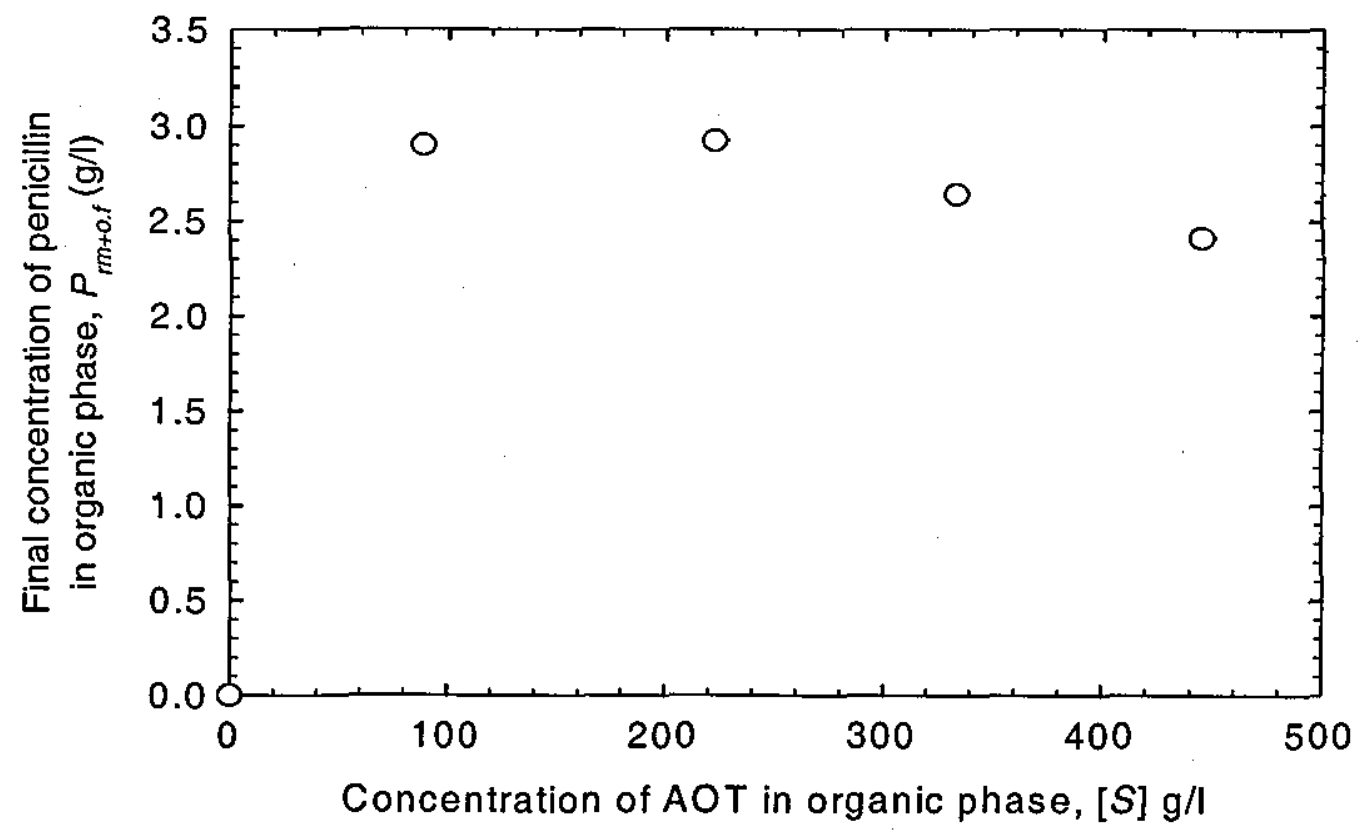

Figure 7 Concentration of penicillin $\mathrm{G}$ in the organic phase at different AOT concentrations. $\left([\mathrm{KCl}]=10 \mathrm{~g} / \mathrm{l}\right.$; temperature $=23 \pm 1^{\circ} \mathrm{C}$; stirring speed $=400 \mathrm{rpm}(10$ $\min ) ; \mathrm{pH}=7.6$; initial penicillin $\mathrm{G}$ concentration in the aqueous phase $=3.6 \mathrm{~g} / \mathrm{l}$.) 


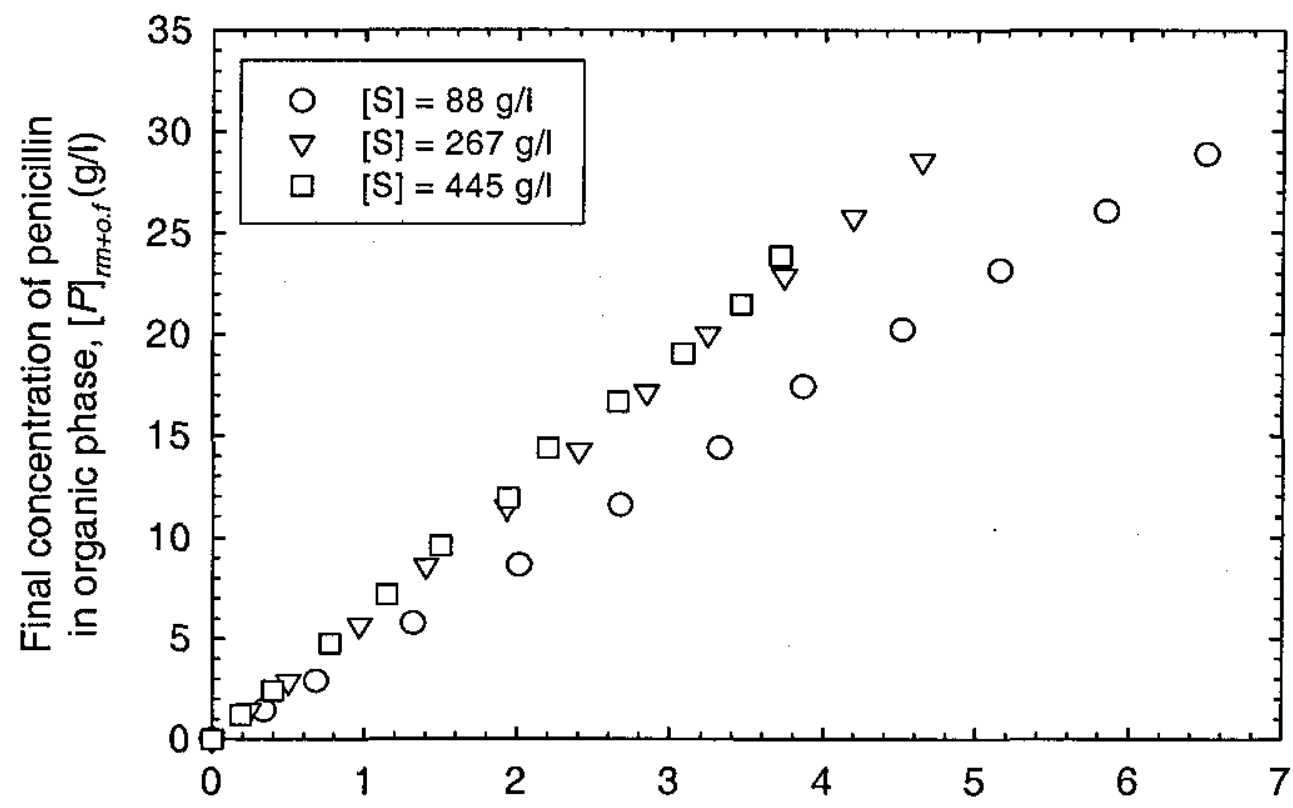

Final concentration of penicillin in aqueous phase, $[P]_{a q . f}(\mathrm{~g} / \mathrm{l})$

Figure 8 The distribution of penicillin $\mathrm{G}$ between the revere micelles in the organic phase, $[P]_{r m+o f f}$, and the aqueous phase, $[P]_{a q . f .}([\mathrm{KCl}]=10 \mathrm{~g} / \mathrm{l}$; temperature $=$ $23 \pm 1^{\circ} \mathrm{C}$; stirring speed $=400 \mathrm{rpm}(10 \mathrm{~min}) ; \mathrm{pH}=7.6$; initial penicillin $\mathrm{G}$ concentration in the aqueous phase $=3.6 \mathrm{~g} / \mathrm{l}$.)

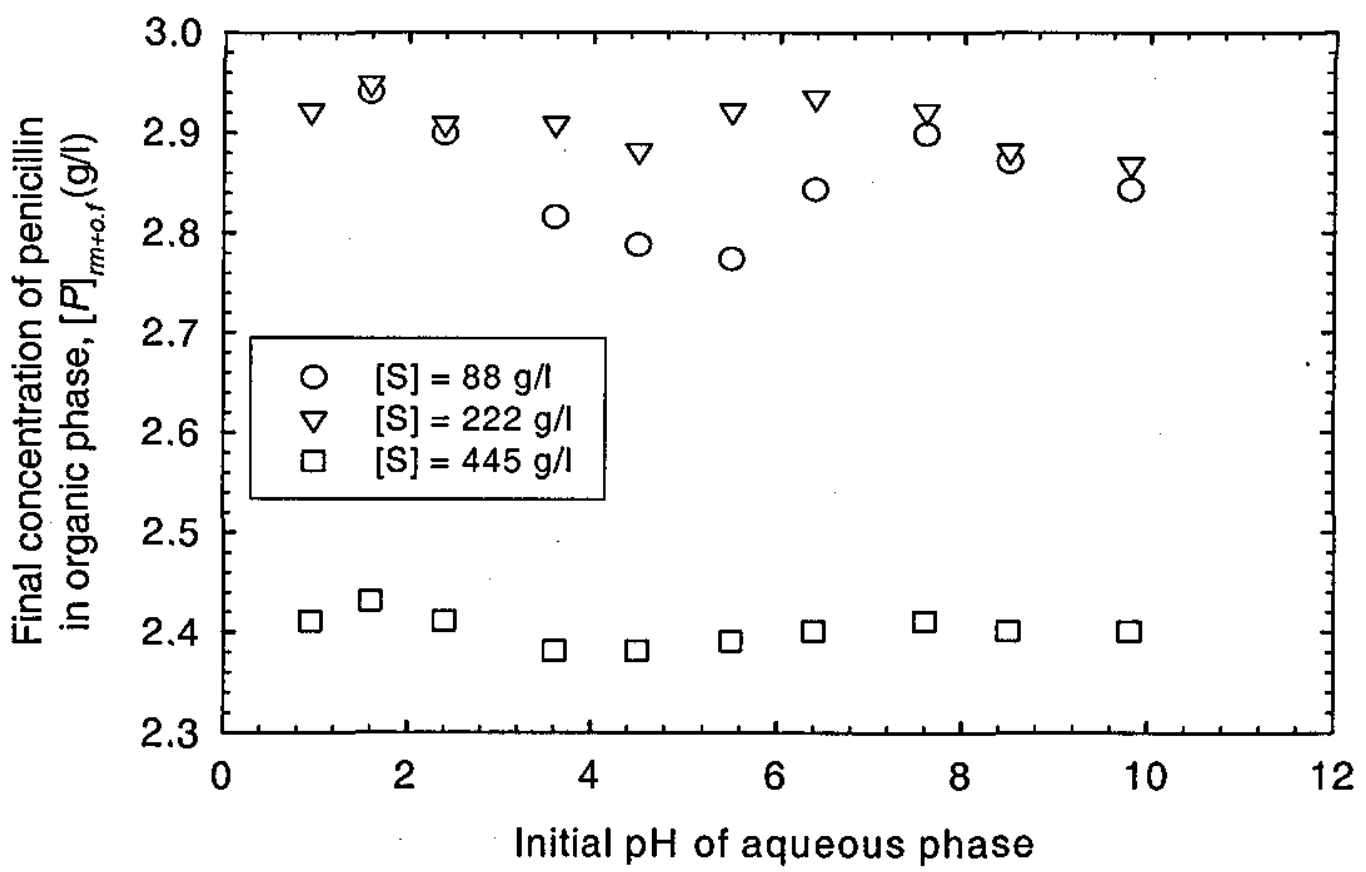

Figure 9 The effects of $\mathrm{pH}$ and AOT concentration on the amount of penicillin extracted into the micellar phases. (Temperature $=23 \pm 1^{\circ} \mathrm{C}$; stirring speed $=400 \mathrm{rpm}$ $(10 \mathrm{~min})$; initial penicillin $\mathrm{G}$ concentration in the aqueous phase $=3.6 \mathrm{~g} / \mathrm{l}$.) 


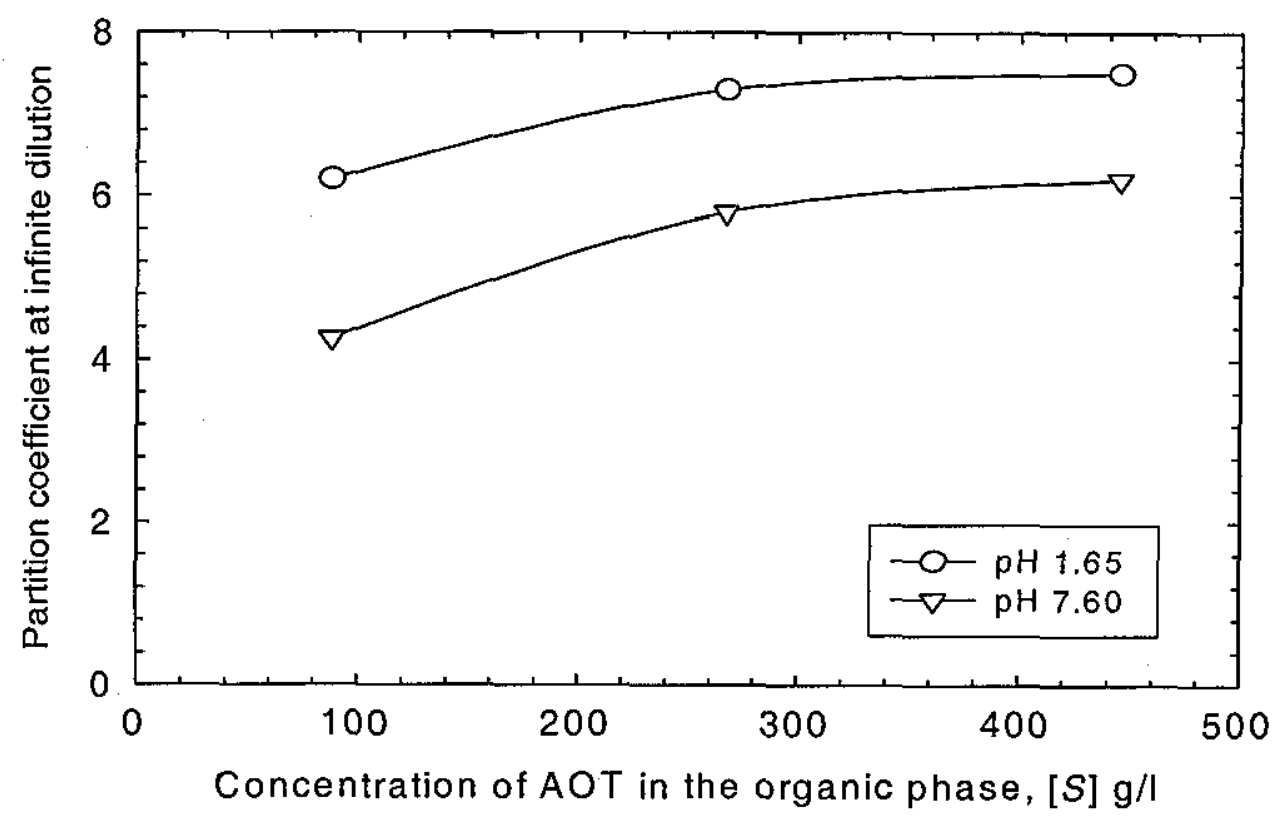

Figure 10 The variation of the partition coefficient at infinite dilution $\left(K^{\infty}\right)$ with AOT concentration and $\mathrm{pH}$. (Temperature $=23 \pm 1^{\circ} \mathrm{C}$; stirring speed $=400 \mathrm{rpm}(10$ $\min$ ); initial penicillin $\mathrm{G}$ concentration in the aqueous phase $=3.6 \mathrm{~g} / \mathrm{l}$.) 

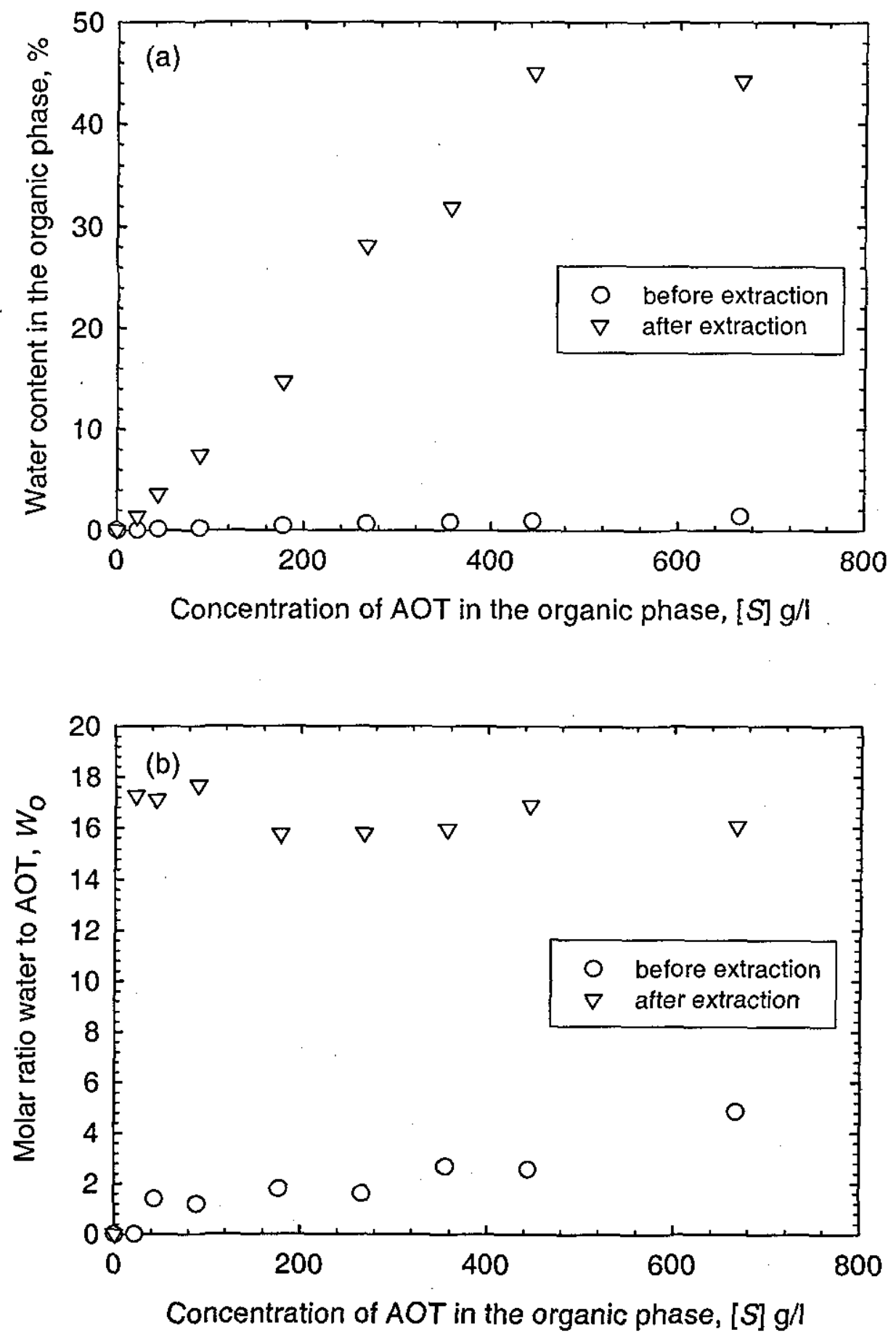

Figure 11 The effect of AOT concentration on water uptake into the reverse micelle (organic) phase presented as (a) water content, and (b) molar ratio of water to AOT, $W_{o} .\left([\mathrm{KCl}]=10 \mathrm{~g} / \mathrm{l} ;\right.$ temperature $=23 \pm 1^{\circ} \mathrm{C} ;$ stirring speed $=400 \mathrm{rpm}(10 \mathrm{~min}) ; \mathrm{pH}=$ 7.6.) 


\title{
6-2 Abstract for the $10^{\text {th }}$ World Filtration Congress (WFC 10), Leipzig, Germany, 14-18 ${ }^{\text {th }}$ April 2008.
}

\section{SEPARATION OF PHARMACEUTICAL PRODUCTS WITH REVERSE MICELLES}

\author{
S.H. Mohd-Setapar*, R.J. Wakeman and E.S. Tarleton \\ Advanced Separation Processes Group, Department of Chemical Engineering, \\ Loughborough University, Loughborough, Leicestershire, LE11 3TU, UK. \\ *Corresponding author email address S.H.Mohd-Setapar@lboro.ac.uk
}

The use of reverse micelle systems for bioseparation has received considerable attention in recent years due to its ability to selectively solubilise solutes from an aqueous phase or from the whole broth and to maintain their biological activities. Some researchers have tried to extract proteins by using the reverse micelle system and have found it to be a very successful. However, very few have used the reverse micelle system for the extraction of other pharmaceutical products. This paper presents results from studies on extraction of penicillin $\mathrm{G}$ from aqueous solutions by employing the principle of liquid-liquid extraction with reverse micelles. An AOT reverse micelle system was used to extract penicillin $\mathrm{G}$ from an aqueous phase (containing $\mathrm{KCl}$ and penicillin $\mathrm{G}$ ) into an organic phase (containing AOT and isooctane). The penicillin $\mathrm{G}$ can be extracted into a reverse micelle solution by manipulating several parameters such as aqueous phase $\mathrm{pH}$, surfactant concentration, and salt concentration and type. Other properties such as CMC and water content of organic phase were shown to influence the solubilisation of penicillin $\mathrm{G}$ into the reverse micellar phase. Among all the parameters understudied, we found that $\mathrm{pH}$ and surfactant concentration were the most important parameters in the solubilisation process. The results show that even though the highest concentration of penicillin $\mathrm{G}$ in the organic phase $\left([P]_{r m+o, f}\right)$ is obtained at $\mathrm{pH} 1.65$, but the initial concentration of penicillin $\mathrm{G}\left([P]_{a q . i}\right)$ used must lower than $10.7 \mathrm{~g} / 1$ at this $\mathrm{pH}$ value. Whilst at $\mathrm{pH} 7.6$, the solubilisation occurred even if using $[P]_{a q . i}$ as high as $35 \mathrm{~g} / \mathrm{l}$. The solubilisation of penicillin $\mathrm{G}$ into reverse micelles increased with increasing surfactant concentration due to the increased number of AOT molecules, which also caused an increase of the volume of the organic phase containing the reverse micelles. The addition of salt is important to avoid emulsion during the extraction process, however high salt concentration reduces the solubilisation of penicillin $G$ into reverse micelles due to a weakening of the electrostatic interaction between penicillin $\mathrm{G}$ and surfactant molecules.

Keywords: Antibiotic, reverse micelles, surfactant, separation, liquid-liquid extraction. 


\title{
6-3 Abstract for the Icheme Symposium - What's New in Fluid Separations?. GlaxoSmithKline, Stevanage, UK, 30 May 2008.
}

\section{SEPARATION OF PENICILLIN G WITH REVERSE MICELLES}

\author{
S.H. Mohd-Setapar ${ }^{*}$, R.J. Wakeman and E.S. Tarleton \\ Advanced Separation Processes Group, Department of Chemical Engineering, \\ Loughborough University, Loughborough, Leicestershire, LE11 3TU, UK.
}

Reverse micelle extraction has received considerable attention in recent years due to its ability to selectively solubilise solutes from an aqueous phase, and in the case of biomolecules to maintain their biological activities. This research investigate the results from studies on extraction of penicillin $\mathrm{G}$ from aqueous solution (forward extraction) and from the reverse micelles to a new aqueous solution (backward extraction). The extraction is influenced by the initial penicillin $\mathrm{G}$ concentration, the salt type and concentration in the aqueous phase, $\mathrm{pH}$, and surfactant concentration. The results show that penicillin is an interfacially active compound that interacts with AOT, with the interfacial association being dependent on both $\mathrm{pH}$ and surfactant concentration. When the concentration ratio $[P]_{a q} /[S]$ is high precipitation of the penicillin occurs. The distribution coefficient favours transfer of the penicillin into the reverse micelles at moderate AOT concentrations. The distribution coefficient at infinite dilution, $K^{\infty}$, is shown to be a function of both $\mathrm{pH}$ and surfactant concentration; similar trends in the value of $K^{\infty}$ were observed at different $\mathrm{pH}$ values; $K^{\infty}$ decreases as the surfactant concentration is increased. Despite successfull results in the forward extraction, the extraction of penicillin $G$ in the backward process is found poor. The overall yield, when taking the forward and backward process together, is consequently lower than might be expected - that is, the penicillin recovered from the backward process is less than the initial amount of penicillin $\mathrm{G}$ present in the aqueous phase before forward extraction $\left(P_{\text {aq.i.fw }}\right)$. 


\title{
6-4 Abstract for the Icheme Forum - Chemical Engineering 2008 - ChemEng08, NEC, Birmingham, UK, 28-30 ${ }^{\text {th }}$ October 2008.
}

\section{PENICILLIN G SEPARATION BY MICROEMULSION}

\author{
S.H. Mohd-Setapar*, R.J. Wakeman and E.S. Tarleton \\ Advanced Separation Processes Group, Department of Chemical Engineering, \\ Loughborough University, Loughborough, Leicestershire, LE11 3TU, UK. \\ *Corresponding author email address S.H.Mohd-Setapar@lboro.ac.uk
}

The use of microemulsion (or known as reverse micelle) for bioseparation has received considerable attention in recent years due to its ability to selectively solubilise solutes from an aqueous phase or from the whole broth and to maintain their biological activities. Some researchers have tried to extract proteins by using the reverse micelle system and have found it to be a very successful. However, very few have used the reverse micelle system for the extraction of other pharmaceutical products. This paper presents results from studies on extraction of penicillin $\mathrm{G}$ from aqueous solutions by employing the principle of liquid-liquid extraction with reverse micelles. An AOT reverse micelle system was used to extract penicillin $\mathrm{G}$ from an aqueous phase (containing $\mathrm{KCl}$ and penicillin $\mathrm{G}$ ) into an organic phase (containing AOT and isooctane). The penicillin $\mathrm{G}$ can be extracted into a reverse micelle solution by manipulating several parameters such as aqueous phase $\mathrm{pH}$, surfactant concentration, and salt concentration and type. Other properties such as CMC and water content of organic phase were shown to influence the solubilisation of penicillin $\mathrm{G}$ into the reverse micellar phase. Among all the parameters understudied, we found that $\mathrm{pH}$ and surfactant concentration were the most important parameters in the solubilisation process. The results show that even though the highest concentration of penicillin $\mathrm{G}$ in the organic phase $\left([P]_{r m+o f}\right)$ is obtained at $\mathrm{pH} 1.65$, but the initial concentration of penicillin $\mathrm{G}\left([P]_{\text {aq. }}\right)$ used must lower than $10.7 \mathrm{~g} / \mathrm{l}$ at this $\mathrm{pH}$ value due to formation of precipitation above this value. Whilst at $\mathrm{pH} 7.6$, the solubilisation occurred even if using $[P]_{\text {aq. } i}$ as high as $35 \mathrm{~g} / \mathrm{l}$. The solubilisation of penicillin $G$ into reverse micelles increased with increasing surfactant concentration due to the increased number of AOT molecules, which also caused an increase of the volume of the organic phase containing the reverse micelles. The addition of salt is important to avoid emulsion during the extraction process, however high salt concentration reduces the solubilisation of penicillin $\mathrm{G}$ into reverse micelles due to a weakening of the electrostatic interaction between penicillin $G$ and surfactant molecules.

Keywords: Antibiotic, reverse micelles, surfactant, separation, liquid-liquid extraction 


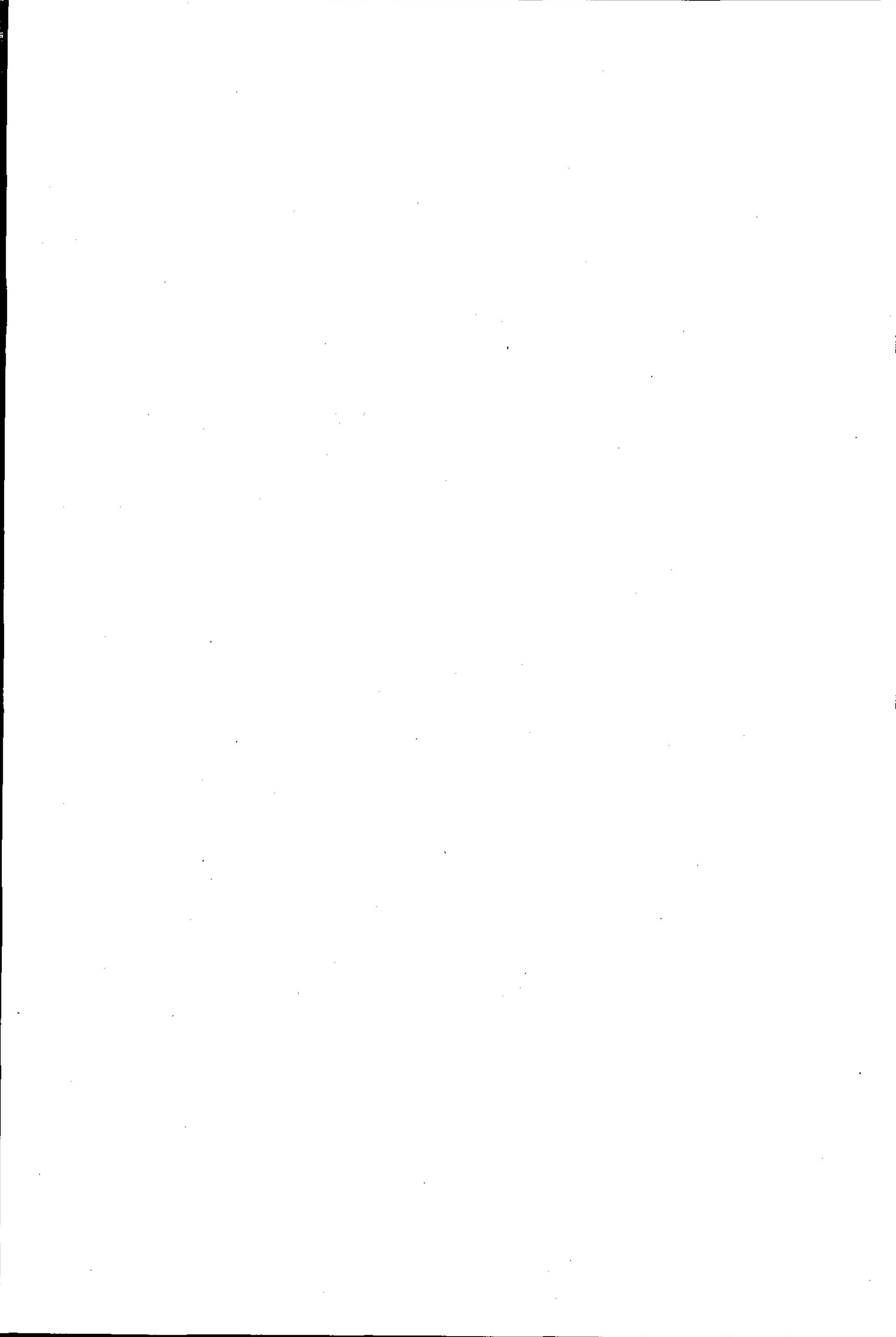


